\title{
Empirische Wirkungsanalyse direkter Transferzahlungen - am Beispiel von Agrarumweltmaßnahmen und der Ausgleichszulage für benachteiligte Gebiete
}

\author{
Dissertation \\ zur Erlangung des Doktorgrades \\ der Fakultät für Agrarwissenschaften \\ der Georg-August-Universität Göttingen
}

\author{
vorgelegt von \\ Andrea Pufahl \\ geboren in Dessau
}

Göttingen, im September 2009 
1. Referent:

Prof. Dr. Bernhard Brümmer

2. Korreferent:

Prof. Dr. Christoph R. Weiss

Tag der mündlichen Prüfung: $\quad$ 11. November 2009 

Teile dieser Dissertation wurden vorab mit Genehmigung veröffentlicht:

\section{Zeitschriftenbeiträge}

PufAHL A, WeIss CR (2009) Evaluating the effects of farm programs: Results from propensity score matching. European Review of Agricultural Economics 36 (1), 79-101

PufAHL A, WeISS CR Effekte agrarpolitischer Programme auf den betrieblichen Faktoreinsatz. Agrarwirtschaft (im Druck)

\section{Tagungsbeiträge}

Pufahl A, WeIss CR (2009) Farm Structure and the Effects of Agri-Environmental Programs: Results from a Matching Analysis for European Countries. Paper contribution to the 111 EAAE-IAAE Seminar 'Small farms: decline or persistence', University of Kent, Canterbury, UK, $26^{\text {th }}-27^{\text {th }}$ June 2009

PUfAHL A, WeIss CR (2008) Evaluating the effects of farm programs: Results from Propensity Score Matching. 12 ${ }^{\text {th }}$ Congress of the European Association of Agricultural Economists - EAAE. Research in Agricultural and Applied Economics: http://agecon.lib.umn.edu/. Stand 27.1.2009

PUfAHL A, WeISS CR (2008) Evaluating the effects of farm programs: results from propensity score matching. In: Bartova, L., M'barek, R. und Ratinger T. (Hrsg.): Modelling agricultural and rural development policies: proceedings, $107^{\text {th }}$ EAAE Seminar, $29^{\text {th }}$ January $-1^{\text {st }}$ February, 2008, Seville. Luxembourg (Office for Official Publications of the European Communities)

\section{Diskussionspapier}

PufaHL A, WeISS CR (2007) Evaluating the effects of farm programs: Results from propensity score matching. Department of Economics Working Paper Series 114. Vienna University of Economics and Business Administration: http://epub.wu-wien.ac.at/wp/. Stand 20.12.2007 


\section{Vorwort}

Die vorliegende Arbeit wurde unter Anleitung von Herrn Prof. Dr. B. Brümmer, Department für Agrarökonomie und Rurale Entwicklung der Georg-August-Universität Göttingen, erstellt. Ihm gilt mein herzlicher Dank für die Betreuung dieser Arbeit. Besonderen Dank schulde ich Herrn Prof. Dr. C. R. Weiss, Institut für Volkswirtschaftspolitik und Industrieökonomik der Wirtschaftsuniversität Wien, für die intensive Betreuung während meines Gastaufenthaltes in Wien und die äußerst gewinnbringende Zusammenarbeit bei der Publikation und Diskussion der Ergebnisse. Herrn Prof. Dr. S. von Cramon-Taubadel, Department für Agrarökonomie und Rurale Entwicklung der Georg-August-Universität Göttingen, danke ich für die Abnahme der Prüfung im Rahmen der Disputation.

Mein Dank gilt auch Herrn Prof. Dr. P. Weingarten (Johann Heinrich von Thünen-Institut in Braunschweig (vTI), Institut für Ländliche Räume) und Herrn PD Dr. M. Petrick (IAMO Halle) für ihre Bereitschaft, die Korreferate im Rahmen der Doktorandenseminare zu übernehmen. Ihre Hinweise haben entscheidend zum Gelingen beigetragen.

Diese Arbeit wäre nicht ohne die Unterstützung meiner Kollegen und Kolleginnen am vTI, Institut für Ländliche Räume und Institut für Betriebswirtschaft, möglich gewesen. Namentlich möchte ich Herrn Dr. W. Kleinhanß und Herrn B. Osterburg danken, die mir den Zugang zu den Datenbeständen des vTI ermöglichten sowie Herrn Prof. Dr. F. Isermeyer, der den ersten Kontakt zu Prof. Dr. B. Brümmer herstellte und mich bei der Beantragung des DAAD-Stipendiums unterstützte. Die Leitung des Institutes für Ländliche Räume, in Person von Herrn Prof. Dr. P. Weingarten und Frau Prof. Dr. S. Hermann, unterstützte zu jeder Zeit die Durchführung der Promotion.

Für die inhaltliche Diskussion und die Hinweise beim redigieren der Arbeit danke ich insbesondere Anne Margarian (vTI, Institut für Betriebswirtschaft), Silke Hüttel (Humboldt-Universität zu Berlin, Department für Agrarökonomie), Barry K. Goodwin (North Carolina State University, Agricultural and Resource Economics) und Hiltrud Nieberg (vTI, Institut für Betriebswirtschaft).

Allen Kolleginnen der Arbeitsgruppe „6-Länder-Evaluation“ möchte ich für ihre Unterstützung bei der Vereinbarkeit von „Beruf und Promotion“ danken. Meine zeitliche Abwesenheit zum Zweck auswärtiger Forschungs- und Vortragsaufenthalte hatte für alle Kolleginnen - insbesondere für Barbara Fährmann und Regina Grajewski - eine höhere Arbeitsbelastung zur Folge, die sie geduldig ertrugen. Bei inhaltlichen, die Promotion betreffenden Fragen standen sie mir stets hilfreich zur Seite.

Die Arbeit ist unter Mithilfe vieler fleißiger Hände entstanden, insbesondere durch die akribische und geduldige Hilfe von Frau Prüsse, Frau Dittrich, Frau Thiede, Frau Gillner und Frau Böttger (vTI, Institut für Betriebswirtschaft) bei dem Layout von Tabellen, 
Abbildungen sowie des Textes. Danken möchte ich auch den Mitarbeiterinnen des Forschungsdatenzentrums in Kiel und Berlin.

Die Arbeit hat maßgeblich von Erfahrungen profitiert, die ich während zweier Auslandsaufenthalte gesammelt habe. Für die freundliche Aufnahme und Unterstützung bei der Organisation der Aufenthalte danke ich Frau Fiala (Wirtschaftsuniversität Wien), Frau Warrick und Prof. Brandt (North Carolina State University) sowie dem Deutschen Akademischen Austauschdienst (DAAD) für die finanzielle Unterstützung.

Mein größter Dank gilt Stephan, der mich während der unzähligen am Schreibtisch verbrachten Wochenend- und Urlaubstage entbehren musste und mir seine moralische und tatkräftige Unterstützung nie versagte. 


\section{Zusammenfassung}

Mit dem Voranschreiten der Reform der Gemeinsamen Agrarpolitik (GAP) gewinnen umweltorientierte, direkte Transferzahlungen an landwirtschaftliche Betriebe zunehmend an Bedeutung. Als Beispiel seien Zahlungen im Rahmen von Agrarumweltmaßnahmen genannt. Vor dem Hintergrund dieser politischen Entwicklung stellt sich die Frage, ob umweltorientierte Transferzahlungen, neben den gewünschten Umweltwirkungen, auch unerwünschte „Nebenwirkungen“ auf die landwirtschaftliche Produktion und den betrieblichen Faktoreinsatz haben. Sollte dies der Fall sein, so wären umweltorientierte Transferzahlungen nicht als produktions- und damit handelsneutral einzuordnen.

In der vorliegenden Arbeit werden die einzelbetrieblichen Effekte zweier agrarpolitischer Programme (Agrarumweltmaßnahmen, Ausgleichszulage für benachteiligte Gebiete) auf den betrieblichen Faktoreinsatz (Boden, Arbeit, Kapital, Vieh, Pflanzenschutz- und Düngemittel) sowie die Produktion (Umsatz) untersucht. Die Analyse basiert auf einer nicht repräsentativen Stichprobe mit über 20.000 buchführenden Betrieben in Deutschland, für die Beobachtungen im Zeitraum von 2000 bis 2005 vorliegen. Für die empirische Analyse werden ein semi-parametrischer Matching-Ansatz (Propensity Score Matching) sowie verschiedene parametrische Regressionsmodelle für Paneldaten (gepooltes Modell, FixedEffekt-Modell, GMM-Modelle) genutzt. Anhand der Anwendung diese Methoden lassen sich deren Vor- und Nachteile für die mikroökonomische Wirkungsanalyse agrarpolitischer Maßnahmen verdeutlichen.

Die Ergebnisse der empirische Analyse zeigen, dass die Teilnahme an Agrarumweltmaßnahmen (AUM) einen positiven Einfluss auf den betrieblichen Bodeneinsatz hat: Die jährliche Wachstumsrate der landwirtschaftlich genutzten Fläche ist in Betrieben mit AUMTeilnahme um etwa $1 \%$ höher als in vergleichbaren Betrieben ohne Teilnahme. Der positive Einfluss der Ausgleichszulage (AZ) auf das betriebliche Flächenwachstum ist vergleichsweise gering und nur über mehrere Jahre betrachtet statistisch signifikant. Der Effekt beider Programme (AUM, AZ) auf das betriebliche Flächenwachstum ist damit deutlich höher als der entsprechende Effekt der finanziell bedeutsameren gekoppelten Direktzahlungen an landwirtschaftliche Betriebe.

Das höhere Flächenwachstum der Teilnehmerbetriebe ist auf die Flächenverbindung der Agrarumweltprämien bzw. der Ausgleichszulage zurückzuführen: Da die Höhe der Programmzahlung primär an den Faktor Boden gebunden ist, scheint die Ausweitung der förderfähigen Flächen eine Strategie der Gewinnmaximierung zu sein: Betriebe wachsen über die Fläche statt über die Erhöhung der Nutzungsintensität. Diese These wird insbesondere für Agrarumweltmaßnahmen untermauert, da sich die Nutzungsintensität (Rinderbesatzdichte, Ausgaben für Düngemittel je Hektar, Arbeitseinsatz je Hektar) und damit auch die Produktivität (Umsatz je Hektar) infolge der Programmteilnahme signifikant verringert. 
Im Mittel aller analysierten Betriebe ist demnach eine extensivierende Wirkung von Agrarumweltmaßnahmen festzustellen, was im Einklang mit den (agrar-)umweltpolitischen Zielen dieser Maßnahmen steht. Der Effekt beider Politikmaßnahmen (AUM, AZ) auf den betrieblichen Umsatz ist nicht signifikant von Null verschieden. Im Durchschnitt aller Betriebe ist somit kein statistisch abgesicherter, produktionsfördernder Effekt der analysierten Programme zu beobachten.

Der positive Effekt der Teilnahme an Agrarumweltmaßnahmen ist als Indiz für den komparativen Vorteil von Teilnehmerbetrieben auf dem Bodenmarkt zu werten. Diese Hypothese wird auch durch Aussagen landwirtschaftlicher Berater gestützt, die die gestiegene Flächennachfrage der AUM-Teilnehmer als ein Hemmnis der betrieblichen Entwicklung für nicht teilnehmende Betriebe ansehen. Inwieweit dieser wachstumsfördernde Effekt den Bodenmarkt bzw. die Entwicklung der Agrarstruktur signifikant beeinflusst, ist auf Basis dieser mikroökonomischen Analyse nicht zu beantworten.

Die empirischen Ergebnisse bestätigen darüber hinaus die These heterogener Effekte der Programmteilnahme an Agrarumweltmaßnahmen und der Ausgleichszulage: Die Höhe der Programmeffekte nimmt mit steigenden Agrarumweltzahlungen bzw. steigender Ausgleichszulage je Betrieb zu. Darüber hinaus beeinflussen betriebsindividuelle Faktoren die Wirkung von Agrarumweltmaßnahmen und der Ausgleichszulage: Betriebe, deren Programmteilnahme aufgrund ihrer individuellen Merkmale (niedrige Nutzungsintensität, geringe Bodenqualität) sehr wahrscheinlich ist, ziehen den höchsten Nutzen aus der Programmteilnahme. Ackerbaubetriebe und Betriebe mit älteren Betriebsleitern weisen unter allen AUM-Teilnehmern das höchste Flächenwachstum auf. Das Flächenwachstum in Ackerbaubetrieben ist auf die ackerbaulich orientierten Modulationsmaßnahmen mit hohen Akzeptanzraten (Mulch- und Direktsaatverfahren, Winterbegrünung, emissionsarme Gülleausbringung) zurückzuführen. Ältere Betriebsleiter nutzen Agrarumweltmaßnahmen teilweise zum ,ausgleiten“ aus der landwirtschaftlichen Erwerbstätigkeit. Die Betriebsgröße und das Bundesland, in dem der Betrieb ansässig ist, scheinen dagegen keinen systematischen Einfluss auf die Höhe der Programmeffekte zu haben.

Neben der empirischen Fragestellung galt ein besonderes Augenmerk der Beurteilung der Eignung der verwendeten Methoden für mikroökonomische Wirkungsanalysen agrarpolitischer Programme. Die Vor- und Nachteile des Matching-Ansatzes gegenüber parametrischen Evaluationsmethoden sind Gegenstand einer aktuellen Diskussion in der Agrarforschung. Vorteile von Matching-Verfahren liegen in den weniger restriktiven Annahmen hinsichtlich der korrekten Spezifikation des empirischen Modells sowie der vergleichsweise einfachen Kommunizierbarkeit der Ergebnisse an ein nicht-wissenschaftliches Publikum. Zu den Nachteilen von Matching-Verfahren zählen die wenig effiziente Nutzung der zur Verfügung stehenden Information und der daraus resultierende große Datenbedarf sowie die eingeschränkten Möglichkeiten zur Überprüfung von Hypothesen. 
Die Auswahl von Referenzbetrieben im Matching-Verfahren erfolgt ausschließlich auf Basis beobachteter Merkmale (selection on observables), während die Selektion in parametrischen Verfahren auch auf Basis unbeobachteter Merkmale (selection on unobservables) erfolgen kann. Die Anwendung des Prinzips selection on unobservables sollte theoretisch zu weniger verzerrten Schätzungen des Programmeffektes führen als die Anwendung des Prinzips selection on observables. Die Ergebnisse der empirischen Analyse lassen jedoch keine dahingehenden Unterschiede zwischen beiden Ansätzen erkennen.

Eine grundsätzliche Unzulänglichkeit mikroökonomischer Analysen besteht in der Erfassung indirekter Effekte. Veränderungen auf Produkt- und Faktormärkten als Folge agrarpolitischer Maßnahmen beeinflussen auch Betriebe, die nicht unmittelbar an dem betrachteten Programm teilnehmen. Eine Berücksichtigung dieser indirekten Effekte in mikroökonomischen Analysen ist nur möglich, wenn eine Einbindung in ein empirisches Modell zur Abbildung des Gesamtsektors erfolgt.

Neben den diskutierten Vor- und Nachteilen der einzelnen Verfahren, sind für die Auswahl der jeweils geeigneten Untersuchungsmethode vor allem praktische Erwägungen relevant. Zu nennen sind hier die zeitliche Varianz der zu untersuchenden Politik, die Beobachtbarkeit von Ergebnissen für Nichtteilnehmer, die Art der zu testenden Hypothesen sowie die Skalierung der Politikvariablen. Im Wissen um die Stärken und Schwächen von nicht-/semi-parametrischen und parametrischen Analysemethoden ist aus der Vielfalt der Methoden der für die zu untersuchende Fragestellung jeweils geeignete Ansatz auszuwählen. 


\section{Inhaltsverzeichnis}

\section{Zusammenfassung}

1 Einleitung 1

1.1 Fragestellung und methodischer Ansatz 2

1.2 Begriffliche Eingrenzung des Forschungsgegenstandes 3

1.3 Aufbau der Arbeit 4

2 Agrarumweltmaßnahmen und die Ausgleichszulage für benachteiligte Gebiete in Deutschland - Ziele, Umsetzung und Bewertungsrahmen $\quad 7$

2.1 Finanzieller, rechtlicher und institutioneller Rahmen $\quad 7$

$\begin{array}{lll}2.2 & \text { Agrarumweltmaßnahmen } & 10\end{array}$

$\begin{array}{ll}\text { 2.2.1 Ziele von Agrarumweltmaßnahmen } & 10\end{array}$

$\begin{array}{ll}\text { 2.2.2 Umsetzung und Akzeptanz von Agrarumweltmaßnahmen } & \\ \text { in Deutschland } & 10\end{array}$

2.2.3 Bewertungsrahmen der EU-KOM für Agrarumweltmaßnahmen 13

$\begin{array}{ll}\text { 2.2.4 Zukunft von Agrarumweltmaßnahmen } & 15\end{array}$

$\begin{array}{lll}2.3 & \text { Ausgleichszulage für benachteiligte Gebiete } & 15\end{array}$

$\begin{array}{lll}\text { 2.3.1 Ziele der Ausgleichszulage } & 17\end{array}$

2.3.2 Umsetzung und Akzeptanz der Ausgleichszulage in Deutschland 17

2.3.3 Bewertungsrahmen der EU-KOM für die Ausgleichszulage $\quad 19$

2.3.4 Zukunft der Ausgleichszulage 20

$\begin{array}{lll}2.4 & \text { Zusammenfassung } & 20\end{array}$

3 Wirkungsmechanismen von direkten Transferzahlungen 22

3.1 Konzeptioneller Rahmen 22

3.2 Einfluss auf den Faktoreinsatz und die Produktion 23

$\begin{array}{lll}3.2 .1 & \text { Ausgleichszulage } & 24\end{array}$

3.3.2 Agrarumweltmaßnahmen 26

3.3 Einfluss auf das außerlandwirtschaftliche Arbeitsangebot 28

$\begin{array}{lll}3.3 .1 & \text { Ausgleichszulage } & 28\end{array}$

3.3.2 Agrarumweltmaßnahmen 29

3.4 Entscheidungsmodell zur Programmteilnahme 29

3.5 Zusammenfassung 32 
4 Wirkungen direkter Transferzahlungen auf den Faktoreinsatz, die Produktion und den außerbetrieblichen Arbeitseinsatz - Stand der Forschung

4.1 Betriebsaufgabe und Anzahl von Betrieben

4.2 Betriebliche Flächenausstattung und Flächenwachstum 37

4.3 Betriebliches und außerbetriebliches Arbeitsangebot 39

4.4 Umweltrelevante Änderungen des Faktoreinsatzes 41

4.5 Produktionseffekte 42

$\begin{array}{lll}4.6 & \text { Zusammenfassung } & 44\end{array}$

5 Methoden für die Evaluation von politischen Programmen 46

$\begin{array}{lll}5.1 & \text { Theorie kausaler Effekte } & 47\end{array}$

5.2 Nicht-experimentelle Schätzer für die Programmevaluation 48

5.2.1 Nicht- und semi-parametrische Schätzer 51

5.2.2 Parametrische Schätzer 53

5.3 Status quo der Wirkungsanalyse direkter Transferzahlungen 57

5.3.1 Nicht- und semi-parametrische Ansätze 59

$\begin{array}{ll}\text { 5.3.2 Parametrische Ansätze } & 61\end{array}$

$\begin{array}{lll}5.4 & \text { Zusammenfassung } & 63\end{array}$

6 Datengrundlagen $\quad 64$

$\begin{array}{lll}6.1 & \text { Buchführungsdaten } & 64\end{array}$

$\begin{array}{lll}6.2 & \text { Sekundärdaten } & 69\end{array}$

$\begin{array}{lll}6.3 & \text { Zusammenfassung } & 70\end{array}$

7 Verwendete Methoden $\quad 71$

$\begin{array}{lll}7.1 & \text { Propensity Score Matching } & 71\end{array}$

$\begin{array}{lll}7.1 .1 & \text { Verwendetes Modell } & 71\end{array}$

$\begin{array}{lll}\text { 7.1.2 Matching-Algorithmen und Matching-Schätzer } & 74\end{array}$

$\begin{array}{ll}\text { 7.1.3 Analyse heterogener Programmeffekte } & 77\end{array}$

$\begin{array}{lll}7.2 & \text { Regressionsmodelle für Paneldaten } & 78\end{array}$

$\begin{array}{lll}7.2 .1 & \text { Verwendete Modelle } & 79\end{array}$

$\begin{array}{lll}\text { 7.2.2 Modellselektion } & 86\end{array}$

$\begin{array}{ll}\text { 7.2.3 Analyse heterogener Programmeffekte } & 89\end{array}$

$\begin{array}{lll}7.3 & \text { Zusammenfassung } & 92\end{array}$ 
8 Empirische Ergebnisse der Matching-Analyse $\quad 93$

8.1 Empirische Spezifikation des verwendeten Matching-Modells 93

8.2 Anwendung des Propensity Score Matching 94

8.3 Effekte der Programmteilnahme für Deutschland 100

8.4 Heterogenität der Effekte der Programmteilnahme 106

$\begin{array}{ll}\text { 8.4.1 Effekte der Programmteilnahme in Abhängigkeit von der } & \\ \text { Höhe der Programmzahlungen } & 106\end{array}$

8.4.2 Effekte der Programmteilnahme nach Regionen 108

8.4.3 Effekte der Programmteilnahme nach Betriebsformen 111

8.4.4 Effekte der Programmteilnahme nach Betriebsgröße 113

$\begin{array}{lll}\text { 8.4.5 Effekte der Programmteilnahme in Abhängigkeit von der } & \\ \text { Teilnahmewahrscheinlichkeit } & 114\end{array}$

$\begin{array}{lll}8.5 & \text { Zusammenfassung } & 116\end{array}$

9 Empirische Ergebnisse der Panelregressionen 117

9.1 Empirische Spezifikation der verwendeten Modelle 117

9.2 Effekt der Programmteilnahme auf das betriebliche Flächenwachstum 121

9.3 Heterogenität der Effekte der Programmteilnahme 130

9.3.1 Effekt der Programmteilnahme in Abhängigkeit von der Höhe der Programmzahlungen 131

9.3.2 Effekt der Programmteilnahme in Abhängigkeit vom Anteil der betrieblichen LF in benachteiligten Gebieten 133

9.3.3 Effekte der Programmteilnahme nach Bundesländern 133

9.3.4 Effekt der Programmteilnahme nach Betriebsformen 135

9.3.5 Effekt der Programmteilnahme nach Betriebsgröße 136

$\begin{array}{lll}\text { 9.3.6 Effekt der Programmteilnahme in Abhängigkeit von der } & \\ \text { Teilnahmewahrscheinlichkeit } & 138\end{array}$

9.3.7 Effekt der Programmteilnahme nach Alter des Betriebsleiters 139

$\begin{array}{lll}9.4 & \text { Zusammenfassung } & 140\end{array}$

10 Diskussion und Ausblick 142

$\begin{array}{lll}10.1 & \text { Empirische Ergebnisse } & 142\end{array}$

10.2 Vor- und Nachteile der verwendeten Methoden 149

$\begin{array}{lll}10.3 & \text { Ausblick und Forschungsbedarf } & 156\end{array}$

$\begin{array}{lr}\text { Literaturverzeichnis } & 159\end{array}$

$\begin{array}{ll}\text { Anhang } & \text { A1-A28 }\end{array}$ 


\section{Verzeichnis der Abbildungen}

Abbildung 1.1: $\quad$ Aufbau der Arbeit

Abbildung 2.1: Zeitliche Entwicklung des rechtlichen und institutionellen

Rahmens von Agrarumweltmaßnahmen und der Ausgleichszulage in Deutschland

Abbildung 2.2: Anteil (Bruttofläche) geförderter Agrarumweltmaßnahmen (2004) an der landwirtschaftlich genutzten Fläche und durchschnittliche AUM-Zahlungen je Betrieb und je Hektar betrieblicher LF

Abbildung 2.3: $\quad$ Umsetzung der Ausgleichszulage 1975 bis 2004: Öffentliche Ausgaben und Anteil der geförderten Fläche an der LF Deutschlands

Abbildung 5.1: Nicht-experimentelle Schätzer in der Programmevaluation

Abbildung 6.1: $\quad$ Verteilung der AUM- und AZ-Zahlungen (2005) in Euro/Hektar LF

Abbildung 8.1: $\quad$ Effekte der AUM- und AZ-Teilnahme auf die Teilnehmer (ATT), Greedy Matching, Deutschland gesamt

Abbildung 8.2: $\quad$ Effekte der AUM- und AZ-Teilnahme auf die Teilnehmer (ATT) differenziert nach Programmzahlungen je Hektar, Greedy Matching

Abbildung 8.3: $\quad$ Effekte der AUM- und AZ-Teilnahme auf die Teilnehmer (ATT), differenziert nach Regionen, Greedy Matching

Abbildung 8.4: $\quad$ Effekte der AUM- und AZ-Teilnahme auf die Teilnehmer (ATT), differenziert nach Betriebsformen, Greedy Matching

Abbildung 8.5: $\quad$ Effekte der AUM- und AZ-Teilnahme auf die Teilnehmer (ATT), differenziert nach Betriebsgröße (ha LF), Greedy Matching

Abbildung 8.6: $\quad$ Einfluss der bedingten Wahrscheinlichkeit der AUM-Teilnahme $p(X)$ auf die Ergebnisänderung $\Delta y_{i}^{1}, \Delta y_{j}^{0}$ (nicht-parametrische Regression), Deutschland gesamt

Abbildung 9.1: $\quad$ Entwicklung der betrieblichen Flächenausstattung (2000 bis 2005) in Betrieben mit und ohne AUM- bzw. AZ-Teilnahme

Abbildung 9.2: $\quad$ Mittelwerte der geschätzten (logarithmierten) Flächenentwicklung in Betrieben mit und ohne AUM- und AZ-Teilnahme 


\section{Verzeichnis der Tabellen}

Tabelle 2.1: $\quad$ Öffentliche Ausgaben für Agrarumweltmaßnahmen und die Ausgleichszulage im EU-Haushaltsjahr 2005

Tabelle 2.2: $\quad$ Förderumfang der Ausgleichszulage: Geförderte LF und Betriebe sowie durchschnittliche Höhe der Ausgleichszulage je Betrieb

Tabelle 3.1: $\quad$ Kategorien direkter Transferzahlungen und deren Wirkungsweise auf die Produktion

Tabelle 4.1: $\quad$ Literaturüberblick - Wirkungen direkter Transferzahlungen auf den betrieblichen Faktoreinsatz, die Produktion und den außerbetrieblichen Arbeitseinsatz

Tabelle 5.1: $\quad$ Vor- und Nachteile von parametrischen Verfahren und Matching-Verfahren

Tabelle 5.2: $\quad$ Literaturüberblick - Zur Wirkungsanalyse direkter

Transferzahlungen verwendete Methoden

Tabelle 6.1: $\quad$ Merkmale der verwendeten Stichprobe der Buchführungsdaten

Tabelle 6.2: $\quad$ Durch LAND-Data (2000) abgebildete Betriebe im Vergleich zur Grundgesamtheit (GG) landwirtschaftlicher Betriebe (1999)

Tabelle 6.3: $\quad$ Verwendete Stichproben der Buchführungsdaten und deren Unterschiede hinsichtlich des Status der Programmteilnahme

Tabelle 6.4: $\quad$ Merkmale der verwendeten Stichprobe der Sekundärdaten

Tabelle 8.1: $\quad$ Parameterschätzwerte der Logit-Modelle zur Erklärung der AUM- und AZ-Teilnahme, Deutschland gesamt

Tabelle 8.2: $\quad$ Vergleich von Mittelwerten (Häufigkeiten) für ausgewählte Variablen im Referenzjahr 2000, Greedy Matching,

Deutschland gesamt

Tabelle 8.3: $\quad$ Effekte der AUM- und AZ-Teilnahme auf die Teilnehmer (ATT), Greedy Matching, Deutschland gesamt

Tabelle 8.4: $\quad$ Effekte der AUM- und AZ-Teilnahme auf die Teilnehmer (ATT), Vergleich verschiedener Matching Schätzer, Deutschland gesamt 105

Tabelle 9.1: $\quad$ Deskriptive Statistik der verwendeten Stichprobe

Tabelle 9.2: $\quad$ Effekt der AUM- und AZ-Teilnahme auf das betriebliche Flächenwachstum

Tabelle 9.3: $\quad$ Mittlerer jährlicher Effekt der AUM- und AZ-Teilnahme auf die betriebliche Flächenentwicklung 
Tabelle 9.4: $\quad$ Effekt der AUM- und AZ-Teilnahme auf das betriebliche Flächenwachstum in Abhängigkeit von der Höhe der Programmzahlungen

Tabelle 9.5: $\quad$ Effekt der AUM- und AZ-Teilnahme auf das betriebliche Flächenwachstum nach Bundesländern

Tabelle 9.6: $\quad$ Effekt der AUM- und AZ-Teilnahme auf die betriebliche Flächenentwicklung, differenziert nach Betriebformen

Tabelle 9.7: $\quad$ Effekt der AUM- und AZ-Teilnahme auf das betriebliche Flächenwachstum in Abhängigkeit von der Betriebsgröße

Tabelle 9.8: $\quad$ Effekt der AUM- und AZ-Teilnahme auf das betriebliche Flächenwachstum in Abhängigkeit von der Wahrscheinlichkeit der Programmteilnahme

Tabelle 9.9: $\quad$ Effekte der AUM- und AZ-Teilnahme nach Alter des Betriebsleiters

Tabelle 10.1: $\quad$ Differenz der mittleren jährlichen Ergebnisänderung (2000 bis 2005, in Prozent) zwischen Betrieben mit und ohne AUM- bzw. AZ-Teilnahme

Tabelle 10.2: $\quad$ Heterogenität der Effekte der AUM- und AZ-Teilnahme in Abhängigkeit von betrieblichen und regionalen Merkmalen 


\section{Abkürzungsverzeichnis}

2SLS, 3SLS

AFP

AK

AKE

ATE

ATT

AUM

$\mathrm{AZ}$

Bl, Blp

CIA

CS

DiD

EU-KOM

EMZ

FE-Modell

GAP

GAK

GG

GL

GMM

GMM-SYS

GMM-OR

GV

$\mathrm{HFF}$

iid

IV-Modelle

LF

$\ln$

Logit-Modell

LVZ

OLS

OR

PA

POLS

PVB

RGV

SB

SUTVA

UHM

$\mathrm{VE}$

$\mathrm{VO}$

WTO
Two-Stage-Least-Squares, Three-Stage-Least-Squares, 2- oder 3stufiges Modell mit Kleinstem-Quadrat-Schätzer

Einzelbetriebliche Agrarinvestitionsförderung

Arbeitskraft, Arbeitskräfte

Arbeitskrafteinheiten

Average Treatment Effect, Mittlerer Effekt der Programmteilnahme

Average Treatment Effect on the Treated,

Mittlerer Effekt der Programmteilnahme auf die Teilnehmer

Agrarumweltmaßnahme(n)

Ausgleichszulage für benachteiligte Gebiete

Betriebsleiter, Betriebsleiterpaar

Conditional Independence Assumption

Common Support Condition

Differenz-in-Differenz

Europäische Kommission

Ertragsmesszahl

Fixed-Effekt-Modell, Panelmodell mit individuenspezifischen und/oder zeitspezifischen festen Effekten

Gemeinsame Agrarpolitik

Gemeinschaftsaufgabe Agrarstruktur und Küstenschutz

Grundgesamtheit

Grünland

General Methods of Moments, Allgemeiner Momentenschätzer

System GMM-Modell

GMM-Modell basierend auf orthogonalen Abweichungen

Großvieheinheiten

Hauptfutterfläche

identically, independently distributed, identisch und unabhängig verteilt

Instrumentvariablen-Modelle

Landwirtschaftlich genutzte Fläche

natürlicher Logarithmus

Logistisches Regressionsmodell

Landwirtschaftliche Vergleichszahl

Ordinary Least Squares, Kleinster-Quadrat-Schätzer

Orthogonal deviations, Orthogonale Abweichungen

Personalaufwand

gepooltes Modell mit Kleinstem-Quadrat-Schätzer

Prozentuale Verringerung des Bias

Raufutter fressendes Großvieh

Standardisierter Bias

Stable Unit Treatment Value Assumption

Unternehmens-Haushalts-Modelle

Vieheinheiten

Verordnung

World Trade Organization, Welthandelsorganisation 


\section{Einleitung}

Unter den direkten Transferzahlungen an landwirtschaftliche Betriebe hat die einheitliche Betriebsprämie finanziell das größte Gewicht. Im Zuge der Reform der Gemeinsamen Agrarpolitik (GAP) gewinnen jedoch umweltorientierte direkte Transferzahlungen immer mehr an Bedeutung. So ist z. B. die Förderung von Agrarumweltmaßnahmen mit der Etablierung der Ländlichen Entwicklungspolitik als Zweite Säule der GAP sprunghaft angestiegen. Ehemals agrarstrukturell orientierte Maßnahmen, wie die Ausgleichszulage für benachteiligte Gebiete (im Folgenden Ausgleichszulage), wurden ebenfalls in den Dienst der Verbesserung und des Erhaltes der Agrarumwelt gestellt.

Vor dem Hintergrund dieser politischen Entwicklung stellt sich die Frage, ob umweltorientierte direkte Transferzahlungen, neben den gewünschten Umweltwirkungen auch unerwünschte „Nebenwirkungen“" auf die landwirtschaftliche Produktion und den betrieblichen Faktoreinsatz entfalten. Sollte dies der Fall sein, so ist die Größenordnung dieser Effekte und deren handelspolitische Implikation abzuschätzen. Die gewonnenen Ergebnisse können genutzt werden, um die bisherige Klassifikation von umweltorientierten Transferzahlungen als weitgehend handelsneutrale Green Box Measures zu verifizieren bzw. zu widerlegen.

Die gesetzlich vorgesehene Bewertung von Agrarumweltmaßnahmen und der Ausgleichszulage erfolgte im Zeitraum 2000 bis 2006 entsprechend der Verordnung zur Entwicklung des Ländlichen Raumes (VO (EG) Nr. 1257/1999). ${ }^{1}$ Der hierfür definierte Bewertungsrahmen der Europäischen Kommission (EU-KOM, 2000) orientiert sich an den maßnahmenspezifischen Zielen der Maßnahmen und fokussiert z. B. ausschließlich auf die Umweltwirkungen von Agrarumweltmaßnahmen. Unerwünschte „Nebenwirkungen“ auf die Produktion oder den gesamtwirtschaftlichen Nutzen werden durch das standardisierte Bewertungsraster der EU-KOM nicht erfasst. Der Evaluationsrahmen der EU-KOM ist thematisch zu eng ausgelegt und lässt wichtige Aspekte für die agrarpolitische Beurteilung von Agrarumweltmaßnahmen und der Ausgleichszulage unberücksichtigt. Um dieses Defizit teilweise $\mathrm{zu}$ beheben, konzentriert sich die vorliegende Arbeit auf die Analyse der Wirkungen von Agrarumweltmaßnahmen und der Ausgleichszulage auf den betrieblichen Faktoreinsatz, die landwirtschaftliche Produktion und den außerbetrieblichen Arbeitseinsatz. Diese Wirkungen wurden bisher nicht systematisch erfasst.

Neben der Frage, welche Wirkungen direkter Transferzahlungen analysiert werden sollten, ist der methodische Ansatz einer solchen Untersuchung von Interesse. Die Folgenabschät-

Die Maßnahmenbewertung im Zeitraum 2007-2013 erfolgt auf Basis der ELER-Verordnung (VO (EG) Nr. 1698/1999) und eines aktualisierten Bewertungsrahmens (EU-KOM, 2006a). 
zung von (ge- und entkoppelten) Direktzahlungen auf das Produktionsvolumen erfolgt in Deutschland primär durch Gleichgewichts- und Optimierungsmodelle (vgl. z. B. KLEINHANß et al., 2003). LEATHERS (1992) zeigt jedoch, dass der Einfluss von agrarpolitischen Instrumenten auf die Agrarstruktur von bestimmten Rahmenbedingungen abhängt und nicht allein aufgrund theoretischer Überlegungen bestimmt werden kann. Somit verbleiben nur empirische, ökonometrische Ansätze zur Analyse struktureller Wirkungen von agrarpolitischen Instrumenten.

Ein weiteres Argument für die wachsende Bedeutung empirischer Evaluationsansätze ist die zunehmende Differenzierung und Komplexität des agrarpolitischen Instrumentariums. Die Effekte von agrarstruktur- und agrarumweltpolitischen Maßnahmen können bisher nur unzureichend in Gleichgewichts- und Optimierungsmodellen abgebildet werden (BRITZ und HeCKELEI, 2008). Gleichzeitig nimmt die finanzielle Bedeutung dieser Maßnahmen zu. In diesem Sinne sind empirische Evaluationsansätze in zweierlei Hinsicht relevant: als eigenständiger Ansatz der Politikbewertung und als „Zulieferer“ der empirischen Basis für die Erweiterung von Gleichgewichts- und Optimierungsmodellen.

\subsection{Fragestellung und methodischer Ansatz}

Wie oben dargestellt, konzentrieren sich die regelmäßig durchgeführten Evaluationen von Agrarumweltmaßnahmen und der Ausgleichszulage auf die Wirkungen, die den von der EU-KOM definierten politischen Zielen der Maßnahmen entsprechen. Eventuell vorhandene unerwünschte „Nebenwirkungen“ dieser Maßnahmen auf die landwirtschaftliche Produktion und die Agrarstruktur wurden bisher kaum untersucht. In der vorliegenden Arbeit wird daher der Frage nachgegangen, welche einzelbetrieblichen Effekte Agrarumweltmaßnahmen und die Ausgleichszulage auf den Faktoreinsatz (Boden, Arbeit, Kapital, Vieh, Pflanzenschutz- und Düngemittel), die Produktion (Umsatz) und die außerbetriebliche Erwerbsbeteiligung des landwirtschaftlichen Haushaltes haben.

Das klassische Problem der Evaluation von (agrar-)politischen Programmen ${ }^{2}$ resultiert aus der Freiwilligkeit der Programmteilnahme: Da sich Betriebe mit und ohne Teilnahme bereits vor der Durchführung der Politik systematisch voneinander unterscheiden, muss die empirische Analyse eine Trennung zwischen dem kausalen Effekt der Programmteilnahme und dem bereits zuvor bestehenden Unterschied zwischen beiden Gruppen, dem Selektionseffekt, vornehmen. Ohne Korrektur des Selektionseffektes wird der kausale Effekt der Programmteilnahme verzerrt gemessen. 
Ein idealisierter Ansatz für die Evaluation von politischen Programmen ist die Durchführung von Experimenten: Durch die zufällige Auswahl der Teilnehmer und Nichtteilnehmer an einem Programm wird eine Gleichverteilung der Merkmale zwischen beiden Gruppen sichergestellt. Der Effekt der Programmteilnahme kann als Mittelwertdifferenz zwischen der Gruppe der Teilnehmer und Nichtteilnehmer ermittelt werden. Aus politischer Sicht wird ein experimenteller Evaluationsansatz kaum akzeptabel sein, da ein Teil der zuwendungsberechtigten Betriebe vorsätzlich von der Förderung ausgeschlossen werden würde. Um den Selektionseffekt bei der Quantifizierung von Programmeffekten zu berücksichtigen, müssen daher so genannte nicht-experimentelle Verfahren verwendet werden (für einen einführenden Überblick vgl. WoOLDRIDGE, 2002: 603). Die Vor- und Nachteile verschiedener nicht-experimenteller Verfahren wurden bereits in der (deutschen) agrarökonomischen Fachliteratur diskutiert (HENNING und MichaleK, 2008a, MARGARIAN, 2008) und stehen in der abschließenden Methodendiskussion im Mittelpunkt.

Das methodische Ziel dieser Arbeit ist die praktische Anwendung und der Vergleich verschiedener nicht-experimenteller Methoden für die Evaluation direkter Transferzahlungen. Ein semi-parametrischer Matching-Ansatz (Propensity Score Matching) und verschiedene parametrische Regressionsmodelle (gepooltes Modell, Fixed-Effekt-Modell, GMMModelle) werden für die Wirkungsanalyse von Agrarumweltmaßnahmen und der Ausgleichszulage verwendet.

\subsection{Begriffliche Eingrenzung des Forschungsgegenstandes}

Unter dem Begriff Wirkungsanalyse sind in dieser Arbeit ex-post Analysen der Auswirkungen von Politiken auf ein interessierendes Ergebnis zu verstehen.

Nach Kuhlmann (1997) sind direkte Transferzahlungen Geldzuweisungen der öffentlichen Hand an Landwirte. Direkte Transferzahlungen können hinsichtlich ihrer Ziele (Einkommensausgleich etc.), Bemessungsgrundlage (Produktionsvolumen, historische Prämienzahlungen etc.) und dem Grad der Entkopplung von der Produktion unterschieden werden (HENRICHSMEYER und WITZKE, 1994, CAHILL, 1997; WTO, 1994). In der agrar(handels-)politischen Debatte der letzten 20 Jahre hat insbesondere das Konzept der Entkopplung an Bedeutung gewonnen. Entkopplung wird dabei als Prozess verstanden, den Grad der Verbindung zwischen agrarpolitischen Instrumenten und der landwirtschaftlichen Produktion zu reduzieren. 
Der Grad der Entkopplung wird dabei maßgeblich von der Bemessungsgrundlage der Zahlung beeinflusst. Die WTO (1994) definiert entkoppelte Einkommensbeihilfen (die so genannten green box measures) als solche, die nicht an das gegenwärtige Produktions- und Preisniveau, den aktuellen Faktoreinsatz oder überhaupt an die Fortführung der Produktion gebunden sind. Diese Definition schließt Instrumente ein, die sowohl positive als auch negative Effekte auf die Produktion haben können. Im Sinne der WTO ist entkoppelt demnach nicht als produktionsneutral zu verstehen, sondern als weniger gekoppelt im Vergleich zu früheren Markt- und Preispolitiken. Die OECD (2001a) empfiehlt nur solche Instrumente als entkoppelt anzusehen, die weder positive noch negative Wirkungen auf die Produktion haben (BALKHAUSEN, 2007).

In dieser Arbeit werden landwirtschaftliche Transferzahlungen entsprechend der WTODefinition klassifiziert. Unterschieden werden gekoppelte und entkoppelte Direktzahlungen, wobei der Grad der Entkopplung unberücksichtigt bleibt. Unter dem Begriff gekoppelte Direktzahlungen werden alle nicht green box-fähigen Instrumente, wie z. B. Marktpreisstützungen, gefasst. Entkoppelte Direktzahlungen beinhalten alle Instrumente, die gegenwärtig als green box Maßnahmen klassifiziert sind. Agrarumweltmaßnahmen und die Ausgleichszulage fallen ebenfalls in die Kategorie entkoppelter Direktzahlungen. Da das Hauptaugenmerk dieser Arbeit auf der Wirkungsanalyse dieser beiden Instrumente liegt, erscheint hier eine weitere Differenzierung sinnvoll: Agrarumweltmaßnahmen in der EU und den USA (Conservation Reserve Programe) werden unter dem Begriff Umweltprogramm zusammengefasst; die Ausgleichszulage für benachteiligte Gebiete wird entsprechend ihrer Ziele als (Agrar-) Strukturprogramm kategorisiert.

\subsection{Aufbau der Arbeit}

Der Aufbau der Arbeit ist schematisch in Abbildung 1.1 dargestellt. Der politische und finanzielle Rahmen von Agrarumweltmaßnahmen und der Ausgleichszulage, sowie deren Umsetzung in Deutschland, ist in im Kapitel 2 beschrieben.

In den Kapiteln 3 bis 5 erfolgt ein Literaturüberblick über die theoretischen, methodischen und empirischen Grundlagen der Wirkungsanalyse von direkten Transferzahlungen. Im Mittelpunkt von Kapitel 3 stehen die Wirkungsmechanismen, über die die Ausgleichszulage und Agrarumweltmaßnahmen die landwirtschaftliche Produktion und den außerlandwirtschaftlichen Arbeitseinsatz beeinflussen. Der Kenntnisstand zu den Wirkungen direkter Transferzahlungen auf den betrieblichen Faktoreinsatz (Boden, Arbeit, Viehbesatz, Dünge- und Pflanzenschutzmitteleinsatz), die Produktion sowie den außerbetrieblichen Arbeitseinsatz ist in Kapitel 4 synoptisch dargestellt. Kapitel 5 gibt einen Überblick über verschiedene Methoden zur Evaluation von politischen Programmen. Ein Vergleich mit den in der agrarökonomischen Forschung verwendeten Methoden zeigt, dass aus metho- 
discher Sicht ein erhebliches Potential besteht, die Evaluation von agrarpolitischen Instrumenten zu verbessern.

Abbildung 1.1: Aufbau der Arbeit

$\underline{\text { Kapitel } 1 \text { Einleitung }}$

Kapitel 2

Umsetzung von Agrarumweltmaßnahmen und der Ausgleichszulage

Literaturüberblick

\begin{tabular}{|c|c|c|}
\hline$\underline{\text { Kapitel } 3}$ & Kapitel 4 & Kapitel 5 \\
\hline Theorie & Empirie & Methodik \\
\hline
\end{tabular}

Daten und Methoden

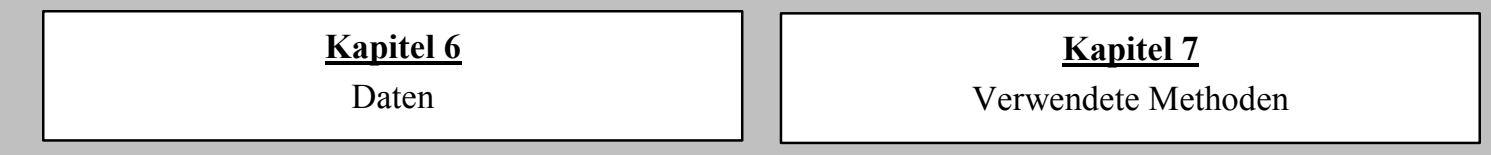

Empirische Ergebnisse

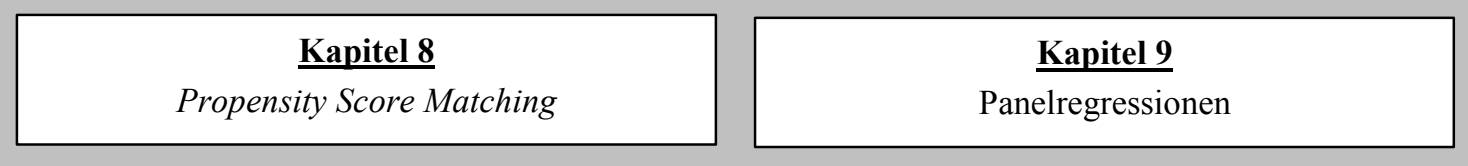

Kapitel 10

Diskussion der Ergebnisse

Quelle: eigene Darstellung.

In den Kapiteln 6 und 7 sind die in der empirischen Analyse verwendeten Datengrundlagen und Methoden beschrieben. Basis der empirischen Analyse ist ein umfangreicher, einzelbetrieblicher Datensatz buchführender Betriebe in Deutschland mit sechs Untersuchungsjahren (2000 bis 2005). Für die Quantifizierung des Effektes von Agrarumweltmaßnahmen und der Ausgleichszulage werden ein semi-parametrisches MatchingVerfahren und verschiedene Panelregressionsmodelle verwendet. Die Methoden sind in Kapitel 7 beschrieben. 
Die Ergebnisse der empirischen Analyse sind in den Kapiteln 8 (Matching-Verfahren) und 9 (Panelregression) dargestellt. Beide Methoden werden auf ein und dieselbe Fragestellung angewendet, um die Ergebnisse sowie die Vor- und Nachteile beider Ansätze vergleichen zu können. Untersucht wird der Einfluss von Agrarumweltmaßnahmen und der Ausgleichszulage auf die Änderung des betrieblichen Faktoreinsatzes (Boden, Arbeit, Kapital, Pflanzenschutz- und Düngemittel, Viehbesatz), auf die außerbetriebliche Erwerbstätigkeit des Betriebsleiterpaares sowie die Produktion (Umsatz) im Zeitraum 2000 bis 2005 in Deutschland. Die Wirkungen von Agrarumweltmaßnahmen und der Ausgleichszulage werden zunächst für die gesamtdeutsche Stichprobe ermittelt. Der Schwerpunkt in Kapitel 8 und 9 liegt jedoch auf der Analyse der Heterogenität der Programmeffekte in Abhängigkeit von betrieblichen und regionalen Merkmalen.

Die empirischen Ergebnisse, die Vor- und Nachteile der verwendeten Methoden sowie der sich aus der Arbeit ergebende Forschungsbedarf werden in Kapitel 10 diskutiert. 


\section{Agrarumweltmaßnahmen und die Ausgleichszulage für benachteiligte Gebiete in Deutschland - Ziele, Umsetzung und Bewertungsrahmen}

Im Mittelpunkt dieses Kapitels stehen die Ziele und die Umsetzung von Agrarumweltmaßnahmen (Kapitel 2.2) und der Ausgleichszulage für benachteiligte Gebiete (Kapitel 2.3) in Deutschland. Der von der EU-KOM vorgegebene Bewertungsrahmen für beide Programme wird vor dem Hintergrund methodischer und empirischer Erfordernisse von Wirkungsevaluationen beleuchtet. Zuvor erfolgt eine Einordnung beider Instrumente in den agrarpolitischen Kontext (Kapitel 2.1). Die Kernaussagen dieses Kapitels werden anschließend zusammengefasst (Kapitel 2.4).

\subsection{Finanzieller, rechtlicher und institutioneller Rahmen}

Der Ausgabenanteil der Ländlichen Entwicklungspolitik an der GAP ist mit rund $14 \%$ (6,8 Mrd. Euro) des Gesamtbudgets relativ gering (vgl. Tabelle 2.1). Etwa $6 \%$ des GAPBudgets bzw. $45 \%$ des Budgets für die Ländliche Entwicklung werden jährlich für Agrarumweltmaßnahmen (AUM) und die Ausgleichszulage (AZ) verausgabt. Diese Zahlen illustrieren, dass beide Maßnahmen zu den Kerninstrumenten der Ländlichen Entwicklungspolitik in der EU zählen.

Tabelle 2.1: $\quad$ Öffentliche Ausgaben für Agrarumweltmaßnahmen und die Ausgleichszulage im EU-Haushaltsjahr 2005

\begin{tabular}{|c|c|c|c|c|c|c|}
\hline \multirow[t]{3}{*}{ Instrument } & \multicolumn{2}{|c|}{ Europa } & \multicolumn{4}{|c|}{ Deutschland } \\
\hline & \multicolumn{2}{|c|}{ EU-Haushalt } & \multirow{2}{*}{$\begin{array}{l}\text { EU-Ausgaben } \\
\text { Mio. Euro }\end{array}$} & \multirow{2}{*}{$\begin{array}{c}\text { Nationale Ausgaben * } \\
\text { Mio. Euro }\end{array}$} & \multicolumn{2}{|c|}{ Ausgaben gesamt } \\
\hline & Mio. Euro & Anteil & & & Mio. Euro & Anteil \\
\hline Gemeinsame Agrarpolitik & 48.928 & $100 \%$ & 7.042 & 1.055 & 8.097 & $100 \%$ \\
\hline Markt- und Preispolitik & 42.101 & $86 \%$ & 5.700 & 0 & 5.700 & $70 \%$ \\
\hline Ländliche Entwicklung & 6.827 & $14 \%$ & 804 & 682 & 1.486 & $18 \%$ \\
\hline Agrarumweltprogramme $* *$ & 2.005 & $4 \%$ & 397 & 236 & 633 & $8 \%$ \\
\hline Ausgleichszulage & 1.124 & $2 \%$ & 141 & 137 & 278 & $3 \%$ \\
\hline
\end{tabular}

* Ausgaben von Bund und Ländern, ** ohne Mittel der fakultativen Modulation.

Quelle: BMELV (2006b), EU-KOM (2006b), EU-KOM (2006c).

Im Jahr 2005 beliefen sich die Ausgaben der Ländlichen Entwicklungspolitik in Deutschland auf 1,486 Mrd. Euro. Etwa 54 \% (804 Mio. Euro) dieser Mittel stellt die EU bereit; die übrigen $46 \%$ (682 Mio. Euro) wurden national, durch Bund und Länder, finanziert. Der überwiegende Anteil dieser Mittel wurde für die Förderung von Agrarumweltmaßnahmen (49\%, 633 Mio. Euro) verausgabt; ein deutlich geringerer Anteil für die Ausgleichszulage (18\%, 278 Mio. Euro). 
Abbildung 2.1 zeigt die zeitliche Entwicklung des rechtlichen und institutionellen Kontextes der Umsetzung von Agrarumweltmaßnahmen und der Ausgleichszulage in Deutschland. Die Beteiligung der EU, der nationalen Ebene und der Bundesländer an der Konzeption und Umsetzung dieser (und anderer) Maßnahmen bezeichnen MEHL und PLANKL (2001) mit dem Begriff „,doppelte Politikverflechtung“. Die Agrarstruktur- und Agrarumweltpolitik der deutschen Bundesländer zeichnete sich bis 1999 durch ein heterogenes „Nebeneinander“ verschiedener Förderprogramme aus. Die Programme basierten auf Vorgaben der EU und/oder des Bundes, umfassten aber auch landeseigene Maßnahmen.

Abbildung 2.1: Zeitliche Entwicklung des rechtlichen und institutionellen Rahmens von Agrarumweltmaßnahmen und der Ausgleichszulage in Deutschland

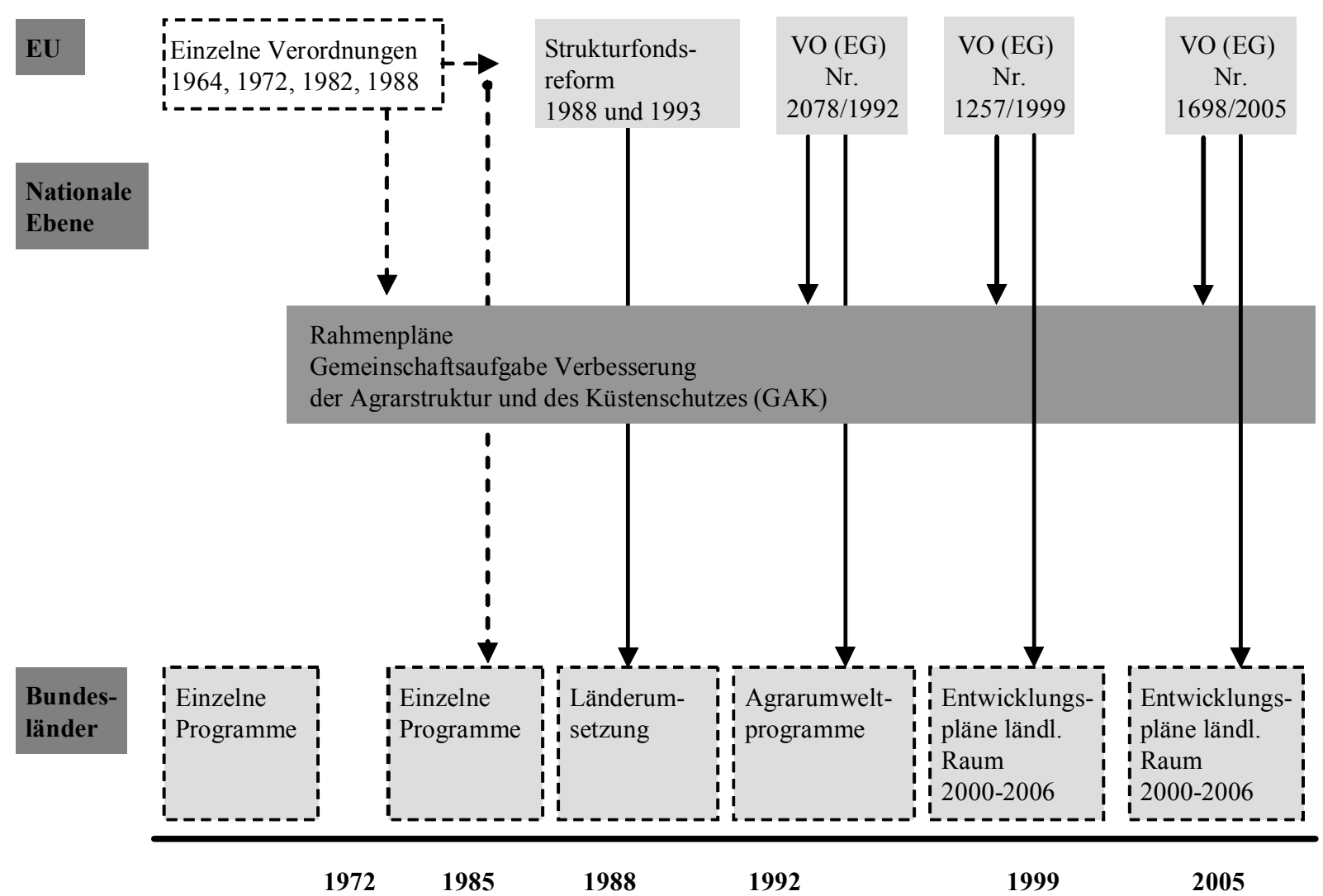

Quelle: ergänzt nach MEHL und PLANKL (2001). 
Eine Bündelungs- und Koordinierungsfunktion zwischen der EU- und der Bundeslandebene übernimmt in Deutschland die Gemeinschaftsaufgabe „Verbesserung der Agrarstruktur und des Küstenschutzes“ (GAK-Gesetz). Der zuständige Planungsausschuss für Agrarstruktur und Küstenschutz (PLANAK) setzt sich aus Vertretern des Bundes und der Länder zusammen. In Form von jährlichen Rahmenplänen legt der PLANAK die Förderinhalte und -bedingungen für die Maßnahmen fest, die deutschlandweit umgesetzt werden (BMVEL, 2000). Zu den GAK-Maßnahmen zählen unter anderem die Ausgleichszulage und ein Teil der Agrarumweltmaßnahmen (Fördergrundsatz Markt- und standortangepasste Landbewirtschaftung). Als Gegenleistung für das Mitspracherecht bei der Ausgestaltung der Förderung beteiligt sich der Bund an der Finanzierung der in den Rahmenplänen enthaltenen GAK-Maßnahmen.

Mit der Agenda 2000 wurden verschiedene Agrarstruktur- und Agrarumweltmaßnahmen in der so genannten „Zweiten Säule“ der GAP gebündelt. Die Konzeption und Umsetzung der Maßnahmen erfolgte auf Basis der Verordnung zur Entwicklung Ländlicher Räume (VO (EG) Nr. 1257/1999). Die Verordnung legte unter anderem die förderfähigen Maßnahmen, deren Ziele und Inhalte für den Planungszeitraum 2000 bis 2006 fest. Die Mitgliedsstaaten konnten aus dem „Maßnahmenmenü“ die für ihre Situation geeigneten Maßnahmen auswählen und in Form von nationalen oder regionalen Entwicklungsprogrammen für den Ländlichen Raum umsetzen.

Die letztendliche Ausgestaltung der Ländlichen Entwicklungspolitik in Deutschland obliegt den Bundesländern. Auf Basis der Vorgaben der EU-KOM und der nationalen GAKRahmenplanung werden die Ländlichen Entwicklungsprogramme für einen siebenjährigen Planungszeitraum erstellt. Die Ländlichen Entwicklungsprogramme müssen von der EUKommission genehmigt werden.

Die Bundesländer übernehmen für Maßnahmen, die nicht im Rahmen der GAK umgesetzt werden, die vollständige nationale Kofinanzierung. Die Kofinanzierungssätze (EU, Bund, Land) sind Verhandlungsgegenstand und variieren zwischen den Maßnahmen und den Bundesländern. Der nationale Kofinanzierungsanteil für Agrarumweltmaßnahmen bzw. die Ausgleichszulage liegt im gesamtdeutschen Mittel zwischen $37 \%$ und $49 \%$ (vgl. Tabelle 2.1). Beide Maßnahmen werden vollständig durch Ausgaben der öffentlichen Hand, das heißt ohne private Beteiligung, finanziert.

Die Mitwirkung der EU bzw. des Bundes an der Ausgestaltung der Ländlichen Entwicklungspolitik in den deutschen Bundesländern führt zu weitestgehend Konflikte minimierenden Verfahrensweisen, z. B. der Gleichbehandlung der Mitgliedsstaaten bzw. Bundesländer und der Orientierung am Status Quo (MenL und PlankL, 2001: 177). Soweit die Mitgliedsstaaten bzw. Bundesländer finanziell dazu in der Lage sind, nutzen sie den verbleibenden Spielraum, um landespolitische Ziele zu akzentuieren. So lag z. B. der 
finanzielle Schwerpunkt der Ländlichen Entwicklungspolitik 2000 bis 2006 in NordrheinWestfalen auf umweltrelevanten Maßnahmen (Agrarumweltmaßnahmen, Ausgleichszulage etc.), wohingegen in Niedersachsen den agrarstrukturellen Maßnahmen (Agrarinvestitionsförderung, Ländlicher Wegebau, Flurbereinigung) ein sehr hohes finanzielles Gewicht eingeräumt wurde (REITER et al., 2003a; 2003b).

\subsection{Agrarumweltmaßnahmen}

Auf europäischer Ebene wurden Agrarumweltmaßnahmen als flankierende Maßnahmen der Mac-Sharry-Reform im Jahr 1992 eingeführt. Bereits vor diesem Zeitpunkt gab es in vielen Bundesländern vergleichbare Maßnahmen (Extensivierungsprogramme), die ausschließlich national finanziert wurden.

\subsubsection{Ziele von Agrarumweltmaßnahmen}

Die Ziele von Agrarumweltmaßnahmen (Untersuchungszeitraum 2000 bis 2005) sind entsprechend Artikel 22 der VO (EG) Nr. 1257/1999 festgelegt. Hierzu gehören:

- die Sicherung einer landwirtschaftlichen Flächenbewirtschaftung, die mit dem Schutz und der Verbesserung der Umwelt ... vereinbar ist,

- die Förderung einer umweltfreundlichen Extensivierung der Landwirtschaft sowie

- der Erhalt bedrohter und wertvoller Kulturlandschaften.

Durch Agrarumweltmaßnahmen werden die bei der Umsetzung umweltfreundlicher Produktionsverfahren entstehenden Kosten kompensiert.

\subsubsection{Umsetzung und Akzeptanz von Agrarumweltmaßnahmen in Deutschland}

Die Maßnahmenteilnahme erfolgt freiwillig und ist an die Einhaltung definierter Umweltauflagen gebunden, die über den Stand der Guten Fachlichen Praxis hinausgehen müssen. Die Teilnahme ist auf landwirtschaftliche Betriebe beschränkt und umfasst zumeist einen fünfjährigen Verpflichtungszeitraum. Abbildung 2.2 stellt die Inanspruchnahme der angebotenen Agrarumweltmaßnahmen in Deutschland im Jahr 2004 dar. 
Abbildung 2.2: $\quad$ Anteil (Bruttofläche ${ }^{3}$ ) geförderter Agrarumweltmaßnahmen (2004) an der landwirtschaftlich genutzten Fläche und durchschnittliche AUMZahlungen je Betrieb und je Hektar betrieblicher LF

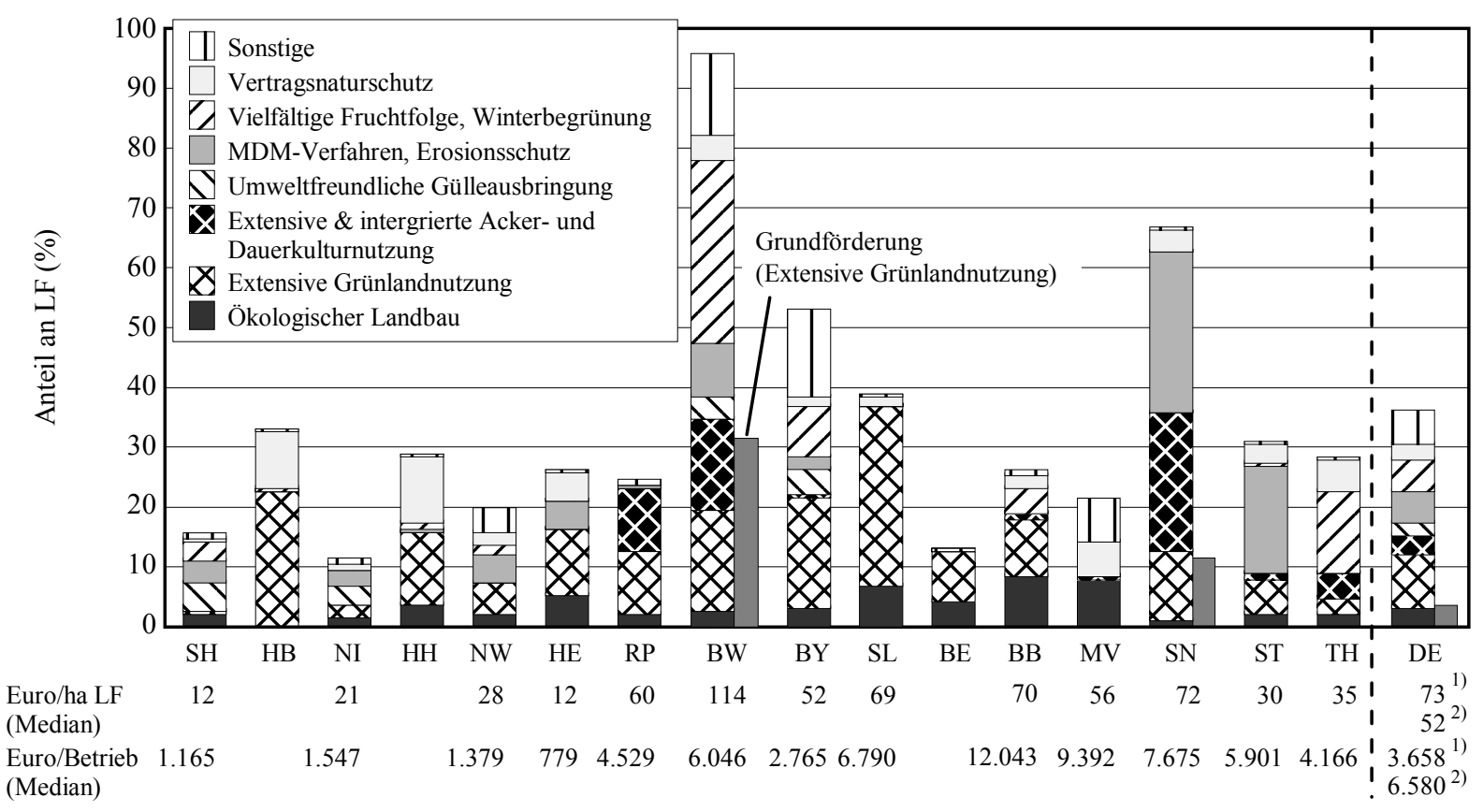

1) Westdeutschland. 2) Ostdeutschland.

$\mathrm{SH}=$ Schleswig-Holstein, $\mathrm{HB}=$ Hansestadt Bremen, NI $=$ Niedersachsen, $\mathrm{HH}=$ Hansestadt Hamburg, $\mathrm{NW}=$ Nordrhein-Westfalen, $\mathrm{HE}=$ Hessen, $\mathrm{RP}=$ Rheinland-Pfalz, $\mathrm{BW}=$ Baden-Württemberg, $\mathrm{BY}=$ Bayern, $\mathrm{SL}=$ Saarland $\mathrm{BE}=$ Berlin, $\mathrm{BB}=$ Brandenburg, $\mathrm{MV}=$ Mecklenburg-Vorpommern, $\mathrm{SN}=$ Sachsen, $\mathrm{ST}=$ Sachsen-Anhalt, $\mathrm{TH}=$ Thüringen, $\mathrm{DE}=$ Deutschland

Quellen: eigene Berechnungen auf Basis von LAND-DATA (Wirtschaftsjahr 2003/04), ART (2005), Doluschitz et al. (2006), FAL, ARUM und BFH (2006), FrEISTAAT THÜRINGEN (2008), IflS (2005), LANDGESELlSCHAFt SACHSEN-ANHALt MBH (2005a; 2005b), N. N. (2005), RAMBøll MANAGEMENT (2005), ZALF (2005a; 2005b).

Extensive und ökologische Bewirtschaftungsweisen auf Grünland und Acker, Mulchsaat-, Direktsaat- und Mulchpflanzverfahren (MDM-Verfahren) sowie die Förderung vielfältiger Fruchtfolgen und von Winterbegrünung haben im Untersuchungszeitraum flächenmäßig die größte Bedeutung in Deutschland. ${ }^{4}$ Die Maßnahmen können sich auf den gesamten Betrieb (Ökologischer Landbau), einen Betriebszweig (Grünlandextensivierung) oder auf Einzelflächen (MDM-Verfahren) beziehen. Die Bundesländer bieten darüber hinaus eine Vielzahl landesspezifischer Maßnahmen an, unter denen der einzelflächenbezogene Vertragsnaturschutz eine hohe Bedeutung hat. Ein Teil der in Abbildung 2.2 dargestellten

3 Die Bruttofläche entspricht der Summe der unter verschiedenen AUM geförderten Flächen, unabhängig davon, ob ein und dieselbe Fläche durch mehrere Maßnahmen gleichzeitig gefördert wird.

4 Die genannten Maßnahmen werden im Rahmen der GAK gefördert. 
Agrarumweltmaßnahmen wurde erst im Jahr 2003, im Zuge der fakultativen Modulation, eingeführt. $\mathrm{Zu}$ diesen so genannten Modulationsmaßnahmen zählen die Förderung der vielfältigen Fruchtfolge, der Winterbegrünung und von MDM-Verfahren sowie die umweltfreundliche Gülleausbringung.

Die durchschnittliche Höhe der Agrarumweltzahlungen ${ }^{5}$ beträgt in Westdeutschland 73 Euro/ha bzw. 4.658 Euro/Betrieb und in Ostdeutschland 52 Euro/ha bzw. 6.580 Euro/Betrieb (Berechnungen auf Basis von LAND-Data). Die in Baden-Württemberg gezahlten Prämien sind fast doppelt so hoch wie im westdeutschen Durchschnitt. Dies ist auf das vielfältige und kombinierbare Maßnahmenangebot zurückzuführen.

Informationen zur Anzahl und Struktur von AUM-Teilnehmern liegen auf Bundesebene nicht vor. ${ }^{6}$ Teilnehmer der Grünlandextensivierung in Nordrhein-Westfalen, Hessen und Niedersachsen sind überwiegend grünlandstarke Futterbaubetriebe. Die Förderung von MDM-Verfahren und der umweltfreundlichen Gülleausbringung wird eher von flächenstarken Marktfrucht- und Veredlungsbetrieben in Anspruch genommen, da sich vor allem in diesen Betrieben die hierfür notwendigen Investitionen in Human- und Sachkapital rentieren (vgl. REITER et al., 2006a; 2006b; 2006c). Aufgrund der bundesweit vergleichbaren Ausgestaltung der erwähnten Maßnahmen sind diese Ergebnisse auf andere Bundesländer übertragbar.

Ein Betrieb wird an einer oder mehreren Agrarumweltmaßnahme(n) nur teilnehmen, wenn der Nutzen der Teilnahme (Einkommen, Wertschätzung des Umweltbeitrages) höher ist als die entstehenden Kosten (Informationsbeschaffung, betriebliche Anpassung). Entscheidende Faktoren hierbei sind die strukturelle Ausgangssituation des Betriebes, das Niveau der zu erfüllenden Umweltauflagen und die Prämienhöhe. Die unter der Annahme durchschnittlicher Produktionsbedingungen kalkulierten Einheitsprämien für Agrarumweltmaßnahmen bedingen eine Konzentration der Inanspruchnahme in weniger intensiv wirtschaftenden Betrieben und Regionen (Osterburg, 2000: 8). Der Anteil der unter Agrarumweltmaßnahmen bewirtschafteten Flächen ist in Ländern mit einem hohen Anteil an Berggebieten und/oder Marginalstandorten groß (Bayern, Baden-Württemberg, Hessen, Nordrhein-Westfalen) und in Gunstregionen, wie Schleswig-Holstein, eher niedrig (Osterburg et al., 1997; SAlHOFer und Glebe, 2006:3).

5 Die Höhe der Agrarumweltzahlungen je Hektar bzw. je Betrieb in Abbildung 2.2 wurde auf Basis von LAND-Data berechnet. Die Höhe der Agrarumweltzahlungen je Hektar entspricht den Agrarumweltzahlungen je Betrieb, bezogen auf die LF des Betriebs. Informationen zur tatsächlich geförderten Fläche und zur Prämienhöhe je Hektar geförderter Fläche liegen keine Informationen vor.

6 Für ökologisch wirtschaftende und mit hoher Wahrscheinlichkeit geförderte Betriebe liegen Informationen in der Agrarstatistik vor (vgl. BICHLER et al., 2005). 
Die Ausgestaltung von Agrarumweltmaßnahmen (Umweltauflagen, Prämienhöhe) bestimmt den Grad der Entkopplung von der Produktion. Aufgrund der Einheitsprämien übersteigen die gewährten Agrarumweltzahlungen die tatsächlichen Opportunitätskosten der Programmteilnahme teilweise deutlich. Ursache hierfür ist, dass die einzuhaltenden Bewirtschaftungsauflagen für die teilnehmenden Betriebe häufig wenig restriktiv sind und ohne nennenswerte betriebliche Anpassungen eingehalten werden können. Je größer die Mitnahmeeffekte von Agrarumweltmaßnahmen, desto höher ist deren Koppelung an die Produktionsentscheidungen des Betriebes. Weiterhin setzt die Teilnahme an Agrarumweltmaßnahmen die Fortführung der Flächenbewirtschaftung voraus. Der Erhalt von Agrarumweltzahlungen ist damit an die Fortführung der Produktion gekoppelt, was der WTO-Definition von entkoppelten Direktzahlungen widerspricht (WTO, 1994).

\subsubsection{Bewertungsrahmen der EU-KOM für Agrarumweltmaßnahmen}

Der inhaltlich-methodische Rahmen für die Evaluation von Agrarumweltmaßnahmen wird durch die Gemeinsamen Bewertungsfragen der EU-Kommission gesetzt (EU-KOM, 2000). Die Bewertungsfragen decken folgende Themenbereiche ab:

- die Verbesserung der Bodenqualität (VI.1.A.), Wasserqualität (VI.1.B.) und Wasserquantität (VI.1.C.),

- den Erhalt bzw. Schutz von Flora, Fauna und Habitaten auf landwirtschaftlichen Flächen (VI.2.A., VI.2.B.) sowie von Landschaften (VI.4.),

- die Sicherung des Fortbestehens gefährdeter Tierrassen oder Pflanzensorten (VI.2.C.).

Ein inhaltlicher Schwachpunkt des Bewertungsrahmens ist die ausschließliche Fokussierung der Bewertungsfragen auf die umweltpolitischen Ziele von Agrarumweltmaßnahmen. Unerwünschte „Nebenwirkungen“" von Agrarumweltmaßnahmen werden in der Evaluation nicht systematisch untersucht und rücken so aus dem Blickfeld der politischen Entscheidungsträger.

Ein methodischer Schwachpunkt des Bewertungsrahmens zeigt sich in den definierten Bewertungsindikatoren. Die Bewertungsindikatoren basieren überwiegend auf outputorientierten Größen, z. B. dem Flächenumfang, auf dem der Einsatz landwirtschaftlicher Produktionsmittel durch Agrarumweltmaßnahmen verringert wurde. Aufgrund der Selbstselektion zur Programmteilnahme ist davon auszugehen, dass sich auf vielen geförderten Flächen der Einsatz landwirtschaftlicher Produktionsmittel durch Agrarumweltmaßnahmen tatsächlich nicht verändert hat. Der Effekt von Agrarumweltmaßnahmen wird durch den Bewertungsindikator demnach verzerrt gemessen. Darüber hinaus lässt der Indikator die Frage nach dem kausalen Zusammenhang zwischen der Verringerung des Einsatzes der 
Produktionsmittel und der Entwicklung von Flora und Fauna bzw. der Boden- und Wasserqualität offen.

Die Wirksamkeit von Agrarumweltmaßnahmen vorausgesetzt, ist deren Nutzen dort am höchsten, wo eine besondere Schutzwürdigkeit bzw. Schutzbedürftigkeit von Umweltmedien (Boden, Wasser etc.) besteht. Eine Untersuchung der „Treffsicherheit“ von Agrarumweltmaßnahmen, ob beispielsweise Erosionsschutzmaßnahmen ausschließlich auf erosionsgefährdeten Flächen gefördert werden, wird weder von der EU-KOM noch von den umsetzenden Mitgliedsstaaten konsequent eingefordert.

Die Kosten von Agrarumweltmaßnahmen werden durch den Bewertungsrahmen der EUKOM nur teilweise erfasst. Die betrieblichen Opportunitäts- und Transaktionskosten der Teilnahme an Agrarumweltmaßnahmen werden pauschal durch standardisierte, flächenbezogene Zahlung aus öffentlichen Mitteln kompensiert. ${ }^{7}$ Unberücksichtigt bleiben bisher die öffentlichen Transaktionskosten, die im Fall von Agrarumweltmaßnahmen eine nicht unbedeutende Kostenkomponente darstellen. Studien aus Nordrhein-Westfalen, Niedersachsen, Hessen und Schleswig-Holstein zeigen, dass der Anteil der öffentlichen Transaktionskosten $^{8}$ an den Gesamtkosten zwischen $10 \%$ (Grünlandextensivierung) und 30\% (Vertragsnaturschutz) liegt (FÄHRMANN UND GRAJEWSKI, 2008, 2008a, 2008b, 2008c).

Die skizzierten inhaltlichen und methodischen Schwachpunkte des Bewertungsrahmens der EU-KOM verdeutlichen den eher technokratischen Evaluationsansatz für Agrarumweltmaßnahmen. Im Vordergrund der Evaluation steht die „Aufaddierbarkeit“ von Indikatoren, über die kein kausaler Zusammenhang zu der gewünschten Wirkung hergestellt werden kann. Aufgrund der unvollständigen Erfassung der Kosten und Wirkungen kann eine Kosten-Wirksamkeits-Analyse, wie sie zur Bewertung von Agrarumweltmaßnahmen notwendig ist (HENRICHSMEYER und WITZKE, 1994), nicht erfolgen.

7 Auf die Nachteile standardisierter Prämiensätze und auf alternative Honorierungsverfahren wird an dieser Stelle nicht eingegangen. Hiefür sei auf Arbeiten von ENGEL et al. (2008), LAND USE POLICY GROUP (2008), LATACZ-LOHMANN und SCHILIZZI (2007) und OFFERMANN et al. (2008) verwiesen.

8 Öffentliche Transaktionskosten der Bundesländer. Die öffentlichen Transaktionskosten auf der Bundesund EU-Ebene wurden nicht berücksichtigt. 


\subsubsection{Zukunft von Agrarumweltmaßnahmen}

Mit der Agrarreform 2003 wurde die Entkopplung der Direktzahlungen und die Bindung der Direktzahlungen an die Einhaltung von Mindeststandards (Cross-Compliance, CC) beschlossen. Die Umsetzung der Entkopplung durch das in Deutschland implementierte Kombimodell mündet in eine regional einheitliche Flächenprämie für Acker und Grünland. Die einzuhaltenden CC-Standards erfordern eine Mindestpflege der durch Direktzahlungen prämierten LF. Damit können Agrarumweltmaßnahmen mit dem Ziel „Erhaltung der Landnutzung/Offenhaltung“" (z. B. Grünlandextensivierung) teilweise obsolet werden, da das Ziel bereits durch die Flächenprämie mit CC-Auflagen erreicht wird (Osterburg et al., 2003: 57).

Die anhaltend hohe Bedeutung von Agrarumweltmaßnahmen in Deutschland wird durch den geplanten Finanzmitteleinsatz für den Zeitraum 2007 bis 2013 untermauert: Vorgesehen sind Ausgaben in Höhe von 4.266 Mio. Euro und damit nur geringfügig weniger als in der Vorperiode. Bezüglich der Umsetzung von Agrarumweltmaßnahmen hat es nur wenige Änderungen gegeben (REITER und ROGGENDORF, 2008). Agrarumweltprogramme gelten entsprechend der WTO-Klassifizierung als green box Instrumente. Einschränkungen im Zuge der Handelsliberalisierung sind daher zunächst nicht zu erwarten.

\subsection{Ausgleichszulage für benachteiligte Gebiete ${ }^{9}$}

Die Einführung der Ausgleichszulage für Berggebiete und bestimmte benachteiligte Gebiete erfolgte im Jahr 1975 durch die Europäische Richtlinie 75/268/EWG. Erklärtes Ziel war der Erhalt der landwirtschaftlichen Erwerbstätigkeit in Gebieten mit ungünstigen Standortbedingungen (Höhenlage, Bodengüte etc.) und damit der Erhalt der landwirtschaftlichen Flächennutzung und einer Mindestbevölkerungsdichte (Artikel 1, 75/268/EWG). Hintergrund der Ausgleichszulage war, dass die landwirtschaftliche Bevölkerung in benachteiligten Gebieten nur in geringem Ausmaß von der GAP profitierte. Befürchtet wurde eine beschleunigte Aufgabe landwirtschaftlicher Betriebe, die bei mangelnden außerlandwirtschaftlichen Erwerbsmöglichkeiten zur Abwanderung der Bevölkerung führen könnte (NEANDER, 1992: 221).

In Deutschland wurde die Ausgleichszulage zunächst nur in Berg- und Kerngebieten (1,5 Mio. ha LF, $11 \%$ der LF Deutschlands) gewährt (PlAnKL und NEANDER, 1994). Im Jahr 1984 ließen Änderungen der GAP Einkommensverluste für landwirtschaftliche Be-

Die Ausgleichszahlung für Gebiete mit umweltspezifischen Einschränkungen wird in dieser Arbeit nicht betrachtet. 
triebe erwarten. Diese sollten teilweise durch die Ausgleichszulage kompensiert werden (Plankl und Neander, 1994: 236). Daraufhin erfolgte eine deutliche Ausweitung der durch die Ausgleichszulage förderfähigen Fläche auf etwa 50 \% der LF Deutschlands (vgl. Abbildung 2.3), ${ }^{10}$ eine Anhebung der Förderhöhen sowie eine Ausweitung der förderfähigen Flächennutzungen auf Ackerland. Bis 1990 verdoppelten sich in Deutschland die öffentlichen Ausgaben für die Ausgleichszulage. ${ }^{11}$ Die Ausgleichszulage wurde so zu einem primär einkommenspolitischen Instrument entwickelt, welches unter anderem in Abhängigkeit von der wirtschaftlichen Lage der landwirtschaftlichen Betriebe gezahlt wird.

Abbildung 2.3: Umsetzung der Ausgleichszulage 1975 bis 2004: Öffentliche Ausgaben (in Euro) und Anteil der geförderten Fläche an der LF Deutschlands

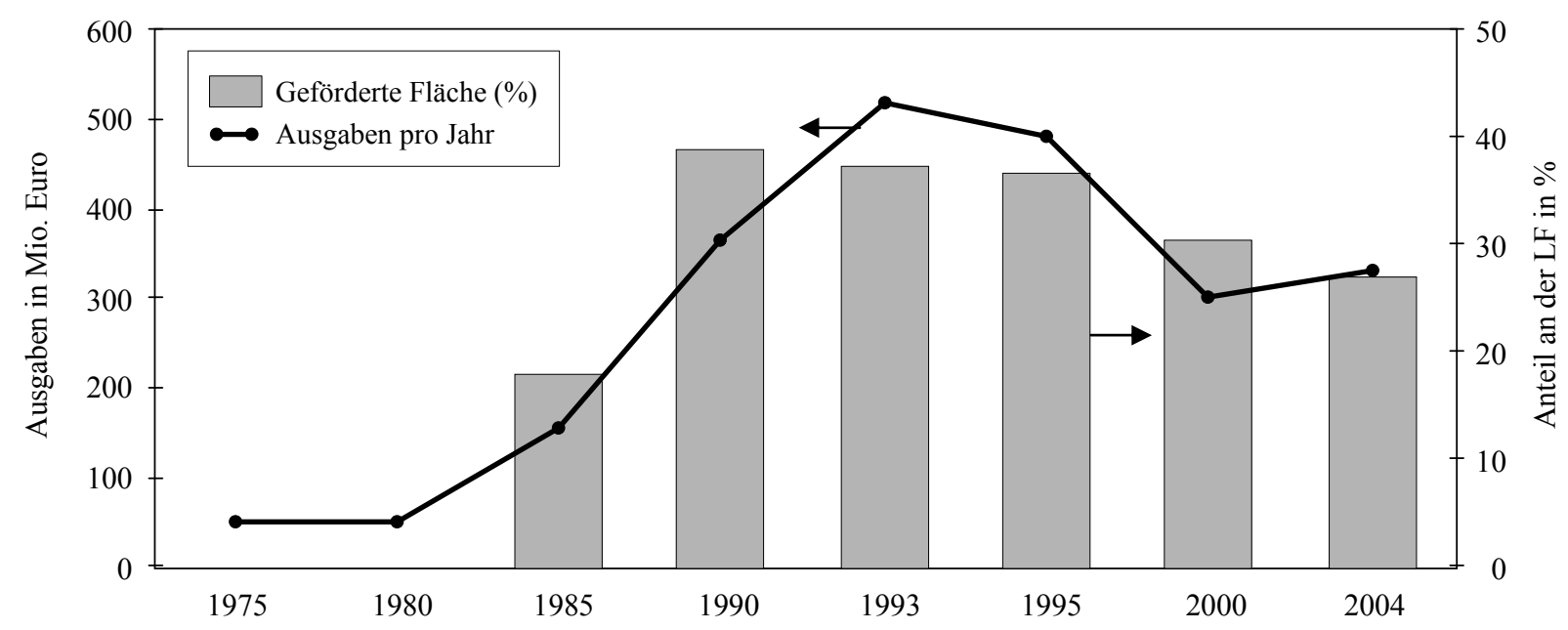

Quelle: GAK-Berichterstattung (div. Jgg.), eigene Bearbeitung.

Die Ausgleichszulage wird in Form einer direkten, an die Flächen gebundenen Zahlung an landwirtschaftliche Betriebe größer drei Hektar gewährt. Im Gegenzug verpflichten sich die Betriebe, die landwirtschaftliche Erwerbstätigkeit ab der ersten Zahlung mindestens fünf Jahre fortzuführen. Diese Verpflichtung entfällt nach Ablauf des ersten fünfjährigen Verpflichtungszeitraums und ist nach dem nunmehr 30-jährigen Bestehen der Ausgleichszulage nicht mehr relevant.

10 Seit 1975 wurde in fast allen Mitgliedsstaaten der EU die Gebietskulisse ausgeweitet (IEEP, 2006: 69). In der EU-weiten Ex-Post-Bewertung der AZ für den Zeitraum 1992 bis 1997 heißt es hierzu: ,, ... there has always been significant political pressure from the Member States to increase the area which can be designated as less favoured areas (AGRAR CEAS CONSULTING, 2003: 93).“ 


\subsubsection{Ziele der Ausgleichszulage}

Die im Untersuchungszeitraum 2000 bis 2005 geltenden Ziele der Ausgleichszulage sind in Artikel 13a der VO (EG) Nr. 1257/1999 dargelegt. Diese umfassen:

- die Sicherung des Fortbestandes der landwirtschaftlichen Bodennutzung und somit Erhaltung einer lebensfähigen Gesellschaftskultur im ländlichen Raum,

- den Erhalt des ländlichen Lebensraums sowie

- die Erhaltung und Förderung nachhaltiger Bewirtschaftungsformen, die insbesondere den Belangen des Umweltschutzes Rechnung tragen.

Gegenüber den landwirtschaftlich orientierten Zielen der Ausgleichszulage (Erhalt der landwirtschaftlichen Beschäftigung und Flächennutzung) erlangen die den ländlichen Raum betreffenden Ziele (Gesellschaftsstruktur, Lebensraum, Umweltschutz) in der VO (EG) Nr. 1257/1999 ein höheres Gewicht. Dies ist primär der Neuorientierung der GAP geschuldet (Erklärung von Cork, 1996). Der Zielerweiterung der Ausgleichszulage folgte jedoch keine veränderte Umsetzung des Instrumentes, z. B. hinsichtlich der Förderkriterien. Insofern werden sich die Wirkungen der Ausgleichszulage nur wenig geändert haben, wenngleich sich diese an geänderten Zielen messen lassen müssen.

Die Angemessenheit der Ziel-Mittel-Auswahl war im Fall der Ausgleichszulage ohnehin immer fragwürdig (vgl. HENRICHSMEYER und WITZKE, 1994). Das (frühere) Ziel des Erhalts einer Mindestbesiedlungsdichte war nur in peripheren Regionen relevant und aufgrund der breiten Streuung der verfügbaren Mittel nicht realistisch. Grundsätzlich ist die Förderung außerlandwirtschaftlicher Arbeitsplätze effizienter als der Erhalt nicht wettbewerbsfähiger Arbeitsplätze in der Landwirtschaft. Landschaftsbezogene Ziele können durch (agrar-)umweltpolitische Maßnahmen besser erreicht werden als durch die Ausgleichszulage.

\subsubsection{Umsetzung und Akzeptanz der Ausgleichszulage in Deutschland}

Die durch die Ausgleichszulage förderfähige Gebietskulisse umfasst etwa $50 \%$ der LF Deutschlands (vgl. Karte A1 im Anhang). Etwa $94 \%$ der Gebietskulisse entfallen auf die Benachteiligte Agrarzone, $4 \%$ auf Berggebiete (überwiegend in Bayern und BadenWürttemberg) und $2 \%$ auf Kleine Gebiete (z. B. die Halligen in Schleswig-Holstein, den Spreewald in Brandenburg). Der tatsächliche Förderumfang der Ausgleichszulage im Jahr 2000 (Flächen) bzw. 2003 (Betriebe) ist aus Tabelle $2.2 \mathrm{zu}$ entnehmen. 
Tabelle 2.2: $\quad$ Förderumfang der Ausgleichszulage: Geförderte LF und Betriebe sowie durchschnittliche Höhe der Ausgleichszulage je Betrieb

\begin{tabular}{|c|c|c|c|c|c|c|c|}
\hline Bundesland & $\begin{array}{c}\text { LF } \\
\text { insgesamt } \\
1999 \\
\text { in } 1.000 \text { ha }\end{array}$ & $\begin{array}{c}\text { Geförderte } \\
\text { LF* } \\
2000 \\
\text { in } 1.000 \text { ha }\end{array}$ & $\begin{array}{l}\text { Anteil } \\
\text { LF } \\
\text { in \% }\end{array}$ & $\begin{array}{c}\text { Betriebe } \\
\text { insgesamt } \\
2003 \\
\text { Anzahl }\end{array}$ & $\begin{array}{c}\text { Geförderte } \\
\text { Betriebe } \\
2003 \\
\text { Anzahl }\end{array}$ & $\begin{array}{c}\text { Anteil } \\
\text { Betriebe } \\
\text { in \% }\end{array}$ & $\begin{array}{c}\text { Ausgleichszulage } \\
\text { je Betrieb*** } \\
2000 \\
\text { Euro/Betrieb }\end{array}$ \\
\hline Schleswig-Holstein & 1.032 & 14 & 1,3 & 19.265 & 402 & 2,1 & 4.572 \\
\hline Bremen & 9 & 5 & 55,2 & 241 & 113 & 46,9 & 2.347 \\
\hline Niedersachsen & 2.661 & 0 & 0,0 & 57.588 & 0 & 0,0 & 0 \\
\hline Hamburg & 14 & 0 & 0,0 & 1.117 & 0 & 0,0 & 0 \\
\hline Nordrhein-Westfalen & 1.502 & 158 & 10,5 & 54.531 & 7.840 & 14,4 & 1.785 \\
\hline Hessen & 766 & 297 & 38,8 & 25.529 & 13.946 & 54,6 & 1.452 \\
\hline Rheinland-Pfalz & 716 & 332 & 46,3 & 29.330 & 8.415 & 28,7 & 2.684 \\
\hline Saarland & 77 & 44 & 57,8 & 1.806 & 933 & 51,7 & 2.592 \\
\hline Baden-Württemberg & 1.473 & 546 & 37,0 & 65.753 & 28.759 & 43,7 & 1.602 \\
\hline Bayern & 3.295 & 1.875 & 56,9 & 135.372 & 81.200 & 60,0 & 1.434 \\
\hline Berlin & 2 & 2 & 106,8 & 103 & 36 & 35,0 & 2.750 \\
\hline Brandenburg & 1.347 & 854 & 63,4 & 6.709 & 3.463 & 51,6 & 7.353 \\
\hline Mecklenburg-Vorpommern & 1.362 & 457 & 33,5 & 5.229 & 2.070 & 39,6 & 10.013 \\
\hline Sachsen & 918 & 281 & 30,6 & 8.132 & 933 & 11,5 & 5.848 \\
\hline Sachsen-Anhalt & 1.173 & 132 & 11,2 & 4.941 & 2.741 & 55,5 & 6.054 \\
\hline Thüringen & 805 & 283 & 35,2 & 5.071 & 865 & 17,1 & 9.869 \\
\hline Deutschland & 17.152 & 4.984 & 30,2 & 388.700 & 141.608 & 36,4 & \\
\hline
\end{tabular}

* Ermittelt nach dem Betriebssitzprinzip (außer in Mecklenburg-Vorpommern). Flächen sind den Ländern zugeschlagen, in denen der Betrieb seinen Sitz hat. ** Betriebe in der Benachteiligten Agrarzone.

Quellen: GAK-Berichterstattung (div. Jgg.), Statistisches Bundesamt (div. Jgg.).

Im Bundesdurchschnitt wird die Ausgleichszulage für etwa ein Drittel der LF Deutschlands, überwiegend für Grünland, gewährt. In Bayern, Brandenburg, Hessen, Berlin, Bremen und im Saarland liegt der Anteil der geförderten Flächen mit mehr als 50 \% der LF deutlich über dem Bundesdurchschnitt. Etwa $36 \%$ aller landwirtschaftlichen Betriebe in Deutschland erhalten für die gesamte oder einen Teil der betrieblichen LF eine Ausgleichszulage.

Der zwischen den Bundesländern variierende Anteil der durch die Ausgleichszulage förderfähigen LF (vgl. Tabelle 2.2, Spalte 3) ist primär auf den unterschiedlichen Anteil der als benachteiligt eingestuften Gebiete zurückzuführen. Darüber hinaus haben die Bundesländer landesspezifische Regelungen getroffen, die die Förderfähigkeit bestimmter Bodennutzungen bzw. Betriebe einschränkt (ausführlich hierzu PlanKL et al., 2005). In Nordrhein-Westfalen, Mecklenburg-Vorpommern und Bremen wird z. B. ausschließlich Grünland durch die Ausgleichszulage gefördert. Niedersachsen und Hamburg haben die Bewilligung der Ausgleichszulage im Jahr 1997 vollständig eingestellt. Schleswig-Holstein gewährt die Ausgleichszulage nur noch in einer reduzierten Gebietskulisse, die die Halligen und küstennahe Gebiete umfasst. 
Die durchschnittliche Ausgleichszulage je Betrieb beträgt etwa 2.000 Euro (Alte Bundesländer) und 7.000 Euro (Neue Bundesländer). Die Prämie je Hektar wird von den Bundesländern in Abhängigkeit von der Flächennutzung (Grünland, Acker), der landwirtschaftlichen Vergleichszahl (LVZ) oder anderen spezifischen Nachteilen (z. B. der Höhenlage des Betriebs) festgelegt. Die bundesdurchschnittlich gezahlte Ausgleichszulage beträgt 68 Euro/ha und variiert zwischen 28 Euro/ha (Brandenburg) und 127 Euro/ha (SchleswigHolstein) (vgl. Abbildung A1 im Anhang). Der Anteil der Ausgleichszulage am Betriebsgewinn liegt je nach Bundesland zwischen $5 \%$ und $23 \%$ (PlankL et al., 2005).

Anhaltspunkte zur Betriebsstruktur der geförderten Betriebe bietet die Agrarstrukturerhebung 1999. In Tabelle 6.2 ist die Struktur von Betrieben innerhalb und außerhalb benachteiligter Gebiete vergleichend dargestellt. In benachteiligten Gebieten sind Betriebe unter 20 ha und Futterbaubetriebe deutlich überrepräsentiert.

\subsubsection{Bewertungsrahmen der EU-KOM für die Ausgleichszulage}

Wie für Agrarumweltmaßnahmen wird der Bewertungsrahmen für die Ausgleichszulage durch die Gemeinsamen Bewertungsfragen der EU-Kommission gesetzt. Die Bewertungsfragen konzentrieren sich auf den Wirkungsbeitrag der Ausgleichszulage

- zum Ausgleich von Einkommensnachteilen (V.1),

- $\quad$ zur dauerhaften Nutzung der LF (V.2),

- zur Erhaltung einer lebensfähigen Gesellschaftsstruktur im ländlichen Raum (V.3) sowie

- $\quad$ zum Schutz der Umwelt (V.4).

Hinsichtlich der inhaltlichen Ausrichtung des Bewertungsrahmens für die Ausgleichszulage gelten die gleichen Kritikpunkte wie für Agrarumweltmaßnahmen: Die Bewertungsfragen orientieren sich an den politischen Zielen der Ausgleichszulage und lassen unerwünschte „Nebenwirkungen“ außer Acht. Dies wird insbesondere am Beispiel der Frage V.3 deutlich, die für Deutschland nicht sinnvoll zu beantworten ist. Die Kosten der Ausgleichszulage werden nur unvollständig erfasst, da die Transaktionskosten nicht berücksichtigt werden.

Ein weiterer methodischer Schwachpunkt des Bewertungsrahmens lässt sich anhand eines Indikators zur Frage V.2 skizzieren. Der Beitrag der Ausgleichszulage zur dauerhaften Nutzung der landwirtschaftlich genutzten Fläche in benachteiligten Gebieten soll anhand der „Veränderung der LF in benachteiligten Gebieten“ gemessen werden. Eine Betrachtung der Veränderung der LF in benachteiligten Gebieten lässt die Frage offen, welche Faktoren für die Änderung der LF insgesamt ausschlaggebend waren und welchen Beitrag 
die Ausgleichszulage an dieser Änderung hat. Der Indikator misst den Effekt der Ausgleichszulage auf den Erhalt der LF verzerrt, da wesentliche Faktoren der Veränderung unbeobachtet bleiben. Der Selektionseffekt wird nicht vom kausalen Effekt der Ausgleichszulage getrennt. Eine vergleichbare Kritik formulierten bereits HENNING und MiCHALEK (2008b).

\subsubsection{Zukunft der Ausgleichszulage}

Die Ausgleichszulage ist in den letzen Jahren zunehmend unter politischen Druck geraten. In Analogie zu den Agrarumweltmaßnahmen wird ein zentrales Ziel der Ausgleichszulage - die Aufrechterhaltung der Flächennutzung - bereits durch die Einführung der CCRegelung und dem damit verbundenen Gebot der Mindestnutzung erreicht. Für eine Fortführung der Ausgleichszulage würde sprechen, wenn das Landnutzungsziel nicht allein durch eine Mindestpflege zu erreichen ist (OSTERBURG et al., 2003: 60) oder alternative Ziele mit der Ausgleichszulage verwirklicht werden sollen. Der EUROPÄISCHE RECHNUNGSHOF (2003) fordert eine Neuabgrenzung benachteiligter Gebiete, die sich stärker als bisher - an der naturbedingten Benachteiligung und weniger an der sozioökonomischen Situation der Gebiete orientieren soll. Die EU-weite Neuabgrenzung der Gebietskulisse ist für 2010 vorgesehen.

Mittlerweile ist in einigen Bundesländern ein leichtes Umsteuern zu erkennen: Als Reaktion auf die EU-seitige Kürzung der Mittel für die Ländliche Entwicklungspolitik in Deutschland (Programmplanungsperiode 2007 bis 2013) wurden in allen Bundesländern die geplanten Ausgaben für die Ausgleichszulage reduziert: Zukünftig stehen jährlich etwa 225 Mio. Euro (und damit etwa 50 Mio. Euro/Jahr weniger als bisher) für die Ausgleichszulage zur Verfügung. Einige Bundesländer ziehen sich aus der Förderung der Ausgleichszulage vollständig zurück (Bremen, Saarland), andere gewähren niedrigere Prämiensätze (Nordrhein-Westfalen) oder erhöhen den landesseitigen Finanzierungsanteil (Bayern) (PoHL, 2008).

\subsection{Zusammenfassung}

Agrarumweltmaßnahmen und die Ausgleichszulage für benachteiligte Gebiete haben innerhalb der Ländlichen Entwicklungspolitik („Zweite Säule“ der GAP), einen hohen finanziellen Stellenwert: Jährlich werden etwa 45 \% (3,1 Mrd. Euro) des EU-Budgets für die Ländliche Entwicklung (ca. 6 \% der GAP), zuzüglich der nationalen Kofinanzierung in etwa der gleichen Höhe, für Agrarumweltmaßnahmen und die Ausgleichszulage verausgabt. Mit der Verordnung zur Entwicklung des Ländlichen Raumes (VO (EG) Nr. 1257/1999) wurden die bis dahin bestehenden Einzelmaßnahmen der EU, der nationalen 
und der regionalen Agrarstruktur- und Agrarumweltpolitik zur so genannten „Zweiten Säule“ der GAP gebündelt. Die VO (EG) Nr. 1257/1999 eröffnet einen weiten Rahmen für die Ausgestaltung der Ausgleichszulage und Agrarumweltmaßnahmen, der in Deutschland von Bund und Ländern ausgefüllt wird: Der Bund übernimmt in Form der GAK eine rahmengebende Kompetenz und einen Teil der nationalen Finanzierung. Den Ländern obliegt die finale Ausgestaltung und Umsetzung der Maßnahmen, entsprechend ihren speziellen Bedürfnissen.

Agrarumweltmaßnahmen wurden auf Europäischer Ebene mit der Mac-Sharry-Reform (1992) eingeführt und dienen der Verwirklichung agrarbezogener Umweltziele. Agrarumweltmaßnahmen zur Förderung extensiver und ökologischer Bewirtschaftungsweisen auf Acker und Grünland haben in Deutschland flächenmäßig die größte Bedeutung. Die durchschnittliche Höhe der Agrarumweltzahlungen je Hektar betriebliche LF bzw. je Betrieb beträgt in Westdeutschland 73 Euro/ha bzw. 4.658 Euro/Betrieb und in Ostdeutschland 52 Euro/ha bzw. 6.580 Euro/Betrieb.

Die Ausgleichszulage für Berggebiete und bestimmte benachteiligte Gebiete wurde im Jahr 1975 und seitdem beständig ausgebaut. Im Verlauf des über 30-jährigen Bestehens sind die ursprünglichen Ziele der Ausgleichszulage, der Erhalt der Flächenbewirtschaftung, der landwirtschaftlichen Erwerbstätigkeit und einer Mindestbevölkerungsdichte, zugunsten des Einkommensziels in den Hintergrund getreten. Etwa ein Drittel der landwirtschaftlich genutzten Fläche Deutschlands wird durch die Ausgleichszulage gefördert. Die Höhe der Ausgleichszulage je Betrieb beträgt durchschnittlich 2.000 Euro (West) bzw. 7.000 Euro (Ost).

Der inhaltlich-methodische Rahmen zur Quantifizierung der Wirkungen von Agrarumweltmaßnahmen und der Ausgleichszulage wird durch die Gemeinsamen Bewertungsfragen der EU-Kommission gesetzt. Anhand ausgewählter Beispiele wurden inhaltliche und methodische Schwachpunkte dieses Bewertungsrahmes aufgezeigt. Ein inhaltlicher Schwachpunkt ist die ausschließliche Orientierung der Bewertungsfragen an den politischen Zielen von Agrarumweltmaßnahmen und der Ausgleichszulage. Nicht intendierte, im politischen Kontext aber durchaus relevante Wirkungen auf die Produktion oder die Agrarstruktur werden somit nicht systematisch erhoben und bleiben bei politischen Entscheidungen unberücksichtigt. Gleiches gilt für die Transaktionskosten dieser Maßnahmen, die bisher nicht erhoben werden. Methodische Schwachpunkte des Bewertungsrahmens sind die Nichtberücksichtigung von Selektionseffekten und die fehlende Kausalität zwischen den geförderten Maßnahmen und den erreichten Wirkungen. 


\section{$3 \quad$ Wirkungsmechanismen von direkten Transferzahlungen}

In diesem Kapitel stehen die Wirkungsmechanismen, über die die Ausgleichszulage und Agrarumweltmaßnahmen die Produktion und den außerlandwirtschaftlichen Arbeitseinsatz des Haushaltes beeinflussen, im Mittelpunkt. In Kapitel 3.1 wird der konzeptionelle Rahmen für die Analyse produktionsrelevanter Effekte von direkten Transferzahlungen vorgestellt. Dieser Rahmen wird im Anschluss genutzt, um die direkten und indirekten Effekte der Ausgleichszulage bzw. von Agrarumweltmaßnahmen auf die Produktion (Kapitel 3.2) und den außerbetrieblichen Arbeitseinsatz (Kapitel 3.3) einzuordnen.

Die Förderung von Agrarumweltmaßnahmen und der Ausgleichszulage ist an bestimmte Bedingungen gebunden, die den Kreis potenzieller Transferempfänger einschränken. Die Wirkungen der Programme, insbesondere von Agrarumweltmaßnahmen, hängen davon ab, welche Betriebe sich für die Teilnahme entscheiden, und welche Faktoren für diese Entscheidung ursächlich sind. In Kapitel 3.4 wird ein Entscheidungsmodell zur Analyse der Bestimmungsgründe der Programmteilnahme vorgestellt.

\subsection{Konzeptioneller Rahmen}

Der Einfluss von agrarpolitischen Instrumenten auf die Produktion kann in direkte und indirekte Effekte unterteilt werden (ANDERSSON, 2004): Direkte Effekte entstehen als Folge der Änderung von Anreizpreisen oder durch die mengenmäßige Begrenzung der Produktion (Preis- und Substitutionseffekte). Indirekte Effekte auf die Produktion sind aufgrund geänderter Einkommen bzw. geänderter Einkommenserwartungen möglich. ANDERSSON (2004) unterscheidet hierbei zwischen Einkommenseffekten, risikobedingten und dynamischen Effekten (vgl. Tabelle 3.1).

Tabelle 3.1: Kategorien direkter Transferzahlungen und deren Wirkungsweise auf die Produktion

\begin{tabular}{|c|c|c|c|c|c|}
\hline & $\begin{array}{c}\text { Preis- } \\
\text { stützung }\end{array}$ & $\begin{array}{l}\text { Subventionen } \\
\text { für Produkte }\end{array}$ & $\begin{array}{l}\text { Subventionen für } \\
\text { variable Inputs }\end{array}$ & $\begin{array}{l}\text { Subventionen } \\
\text { für Boden }\end{array}$ & $\begin{array}{c}\text { Subventionen basierend } \\
\text { auf historischen Anprüchen }\end{array}$ \\
\hline \multicolumn{6}{|l|}{ Direkte Effekte } \\
\hline Preis- und Substitutionseffekte & $\bullet$ & $\bullet$ & $\bullet$ & $\bullet$ & \\
\hline \multicolumn{6}{|l|}{ Indirekte Effekte } \\
\hline Einkommenseffekte & $\bullet$ & $\bullet$ & $\bullet$ & $\bullet$ & $\bullet$ \\
\hline Risikobedingte Effekte & $\bullet$ & $\bullet$ & $\bullet$ & $\bullet$ & $\bullet$ \\
\hline Dynamische Effekte & $\bullet$ & $\bullet$ & $\bullet$ & $\bullet$ & $\bullet$ \\
\hline
\end{tabular}

Quelle: nach OECD (2001b) und ANDERSSON (2004). 
Für die Darstellung der theoretisch zu erwartenden Produktionswirkungen von Transferzahlungen ist die Unterscheidung in gekoppelte und entkoppelte Direktzahlungen, wie sie in Kapitel 1.2 vorgenommen wurde, zu allgemein. Weder gekoppelte noch entkoppelte Direktzahlungen wirken vollständig produktionsneutral. Die OECD (2001b) unterscheidet Transferzahlungen entsprechend ihrer Bemessungsgrundlage und damit hinsichtlich der Wirkungsmechanismen, über die die Produktionsentscheidungen von Betrieben beeinflusst werden.

In Anlehnung an die OECD sind in Tabelle 3.1 Preisstützungsmaßnahmen, Produktsubventionen, Subventionen für fixe (Boden) und variable Faktoren sowie auf historischen Prämiensprüchen basierende Zahlungen unterschieden. Einen direkten und indirekten Einfluss auf die Produktion haben Subventionen der ersten vier Kategorien, da diese an aktuelle Preise, an die aktuelle Produktion oder an den aktuellen Faktoreinsatz gebunden sind. Im Sinne der WTO (1994) sind diese Instrumente überwiegend als gekoppelte Direktzahlungen einzuordnen. Subventionen, die auf historisch erworbenen Prämienansprüchen basieren, wirken vorrangig indirekt auf die Produktion und werden von der WTO als gekoppelte Direktzahlungen betrachtet.

Agrarumweltzahlungen sowie die Ausgleichszulage gelten im Sinne der WTO ebenfalls als entkoppelte Direktzahlungen; nach der OECD (2001b) sind allerdings beide Instrumente als flächengebundene Subventionen einzuordnen, deren Erhalt an bestimmte Bedingungen geknüpft ist. Produktionsrelevante Effekte von Agrarumweltmaßnahmen und der Ausgleichszulage sind aufgrund der Kopplung der Zahlungen an den aktuellen Faktoreinsatz (Boden) theoretisch möglich und maßgeblich von den Bedingungen der Inanspruchnahme beider Programme abhängig. Unter Berücksichtigung dieser Teilnahmebedingungen werden im Folgenden die theoretisch zu erwartenden Wirkungen der Ausgleichszulage und von Agrarumweltmaßnahmen auf die Produktion und den außerbetrieblichen Arbeitseinsatz dargestellt.

\subsection{Einfluss auf den Faktoreinsatz und die Produktion}

Für mikroökonomische Analysen zum Effekt von direkten Transferzahlungen auf die Produktion wird häufig ein Modell mit Mehr-Produkt-Technologie verwendet (Moro und SckoKai, 1999; PeErlings und Poelman, 2004). Unterstellt wird ein gewinnmaximierendes Verhalten der Produzenten und die Abwesenheit von Unsicherheit. Der Gewinn ist eine Funktion der verwendeten Technologie, die durch einen Netput-Vektor mit In- und Outputs repräsentiert wird, und eines Preisvektors für die eingesetzten In- und Outputs. Der Gewinn ist darüber hinaus von der Höhe der Direktzahlungen abhängig. Unter den gegebenen Preisen und Direktzahlungen muss der Produzent eine optimale Entscheidung hinsichtlich der Menge der Netputs und der Verwendung von Land für die Produktion ein- 
zelner Güter treffen (CHAMBERS und Just, 1989). Die Direktzahlungen (und Preise) können also sowohl die produzierte Menge der einzelnen Güter als auch den Umfang der Anbaufläche für jedes produzierte Gut beeinflussen.

\subsubsection{Ausgleichszulage}

Zahlungen im Rahmen der Ausgleichszulage bzw. von Agrarumweltmaßnahmen werden je Hektar landwirtschaftlicher Fläche gewährt. Die Förderung der Ausgleichszulage ist räumlich auf benachteiligte Gebiete begrenzt, erfordert jedoch keine Einhaltung restriktiver Bewirtschaftungsauflagen. Der Erhalt der Ausgleichszulage ist für den geförderten Betrieb mit keinen zusätzlichen Kosten verbunden. Die Ausgleichszulage hat daher den Charakter einer flächengebundenen Subvention.

Flächengebundene Subventionen verringern die Kosten des im Betrieb eingesetzten Bodens. Unter der Annahme der (begrenzten) Substituierbarkeit einzelner Produktionsfaktoren wird ein gewinnmaximierender Betrieb den Einsatz eines sich verbilligenden Faktors (Boden) relativ stärker ausdehnen als den Einsatz der übrigen, nicht subventionierten Faktoren (GUYOMARD et al., 2004; GOHIN et al. 2000; OECD, 2001b). Im Zuge der Flächenausdehnung wird der Einsatz komplementärer Faktoren (Arbeit, Kapital etc.) ebenfalls zunehmen. Aufgrund des überproportionalen Flächenwachstums, wird der Faktoreinsatz je Flächeneinheit reduziert, was zu einer Verringerung der Erträge führen kann (KOESTER, 1992). Empirisch konnte diese Hypothese noch nicht ausreichend untermauert werden (OECD, 2006).

Einzelbetrieblich schaffen flächengebundene Subventionen einen Anreiz zur Ausdehnung der bewirtschafteten Fläche. Da das Bodenangebot insgesamt begrenzt ist, kann die landwirtschaftlich genutzte Fläche (einer Volkswirtschaft) nicht bzw. nur marginal ausgedehnt werden. Aus diesem Grund haben flächengebundene Subventionen einen deutlich geringeren Produktionseffekt als z. B. Subventionen für variable Inputs mit hoher Angebotselastizität (OECD, 2001b).

Der durch die Ausgleichszulage geschaffene Anreiz zur Ausdehnung der betrieblichen LF könnte theoretisch zu einer Verlagerung von Flächen von nicht geförderten hin zu geförderten Betrieben führen. Der Erhöhung der betrieblichen LF durch Pacht bzw. Kauf förderfähiger Flächen sind jedoch Grenzen gesetzt: Die Ausgleichszulage wird nur innerhalb einer definierten Gebietskulisse und hier wiederum nur für bestimmte Bodennutzungen (Grünland, andere extensive Bodennutzungen) gewährt. Diese Begrenzung hat zur Folge, dass die maximal förderfähige Fläche relativ fix ist. Eine Ausweitung der förderfähigen Fläche ist durch eine Änderung der Bodennutzung bzw. den Flächentransfer in förderfähi- 
ge Betriebe ${ }^{12}$ theoretisch möglich, hat jedoch in der Praxis (vermutlich) eine eher geringe Bedeutung.

\section{Indirekte Effekte}

Die Ausgleichszulage hat zunächst einen unmittelbaren Einkommenseffekt. Hierdurch wird ein Anreiz geschaffen im Sektor zu verbleiben. Je nach Verwendung verändert die Ausgleichszulage das Spar- bzw. Konsumverhalten des Haushaltes oder betriebliche Produktions- und Investitionsentscheidungen.

Transferzahlungen verändern auch die Erwartungshaltung von risikoaversen Produzenten gegenüber zukünftigen Einkommen. Direkte Einkommensübertragungen erhöhen das Haushaltsvermögen. In einer Situation mit Unsicherheiten steigt mit zunehmendem Vermögen die Bereitschaft, risikobehaftete und produktionssteigernde Entscheidungen zu treffen (Vermögenseffekt). Ist die Höhe der Transferzahlungen auch vom Grad der Unsicherheit (z. B. vom Preisniveau) abhängig, so tritt zusätzlich ein Versicherungseffekt auf. Der Betrieb wird risikoreichere Produktionsentscheidungen treffen, da mögliche Einkommensverluste (ganz oder teilweise) durch höhere Transferzahlungen kompensiert werden. Der Versicherungseffekt trägt zur Stabilisierung des Einkommens bei (vgl. HENNESSY, 1998).

Vermögenseffekte infolge der Ausgleichszulage sind im Durchschnitt aller Betriebe eher nicht zu erwarten, da die Höhe der Ausgleichszulage je Betrieb verhältnismäßig gering ist (vgl. Tabelle 2.2 in Kapitel 2.3.2). Eine Ausnahme stellt die Ausgleichszulage in Schleswig-Holstein dar, die mit durchschnittlich 125 Euro je Hektar LF die höchste in Deutschland ist. In Einzelfällen sind Vermögenseffekte infolge der Ausgleichszulage daher möglich. Ein Versicherungseffekt ist nicht zu erwarten, da die Höhe der Ausgleichszulage bereits im Vorfeld der Teilnahme fixiert wird.

Betriebe mit unzureichendem Zugang zum Kapitalmarkt erhalten durch Transferzahlungen finanzielle Mittel, um Investitionen zu tätigen oder ihre Kreditwürdigkeit zu verbessern. Dieser Liquiditätseffekt zählt zu den indirekten, dynamischen Effekten von Transferzahlungen (vgl. Andersson, 2004). Die Ausgleichszulage (in Deutschland) hat, im Vergleich zu den Zahlungen der Ersten Säule der GAP, einen geringeren Liquiditätseffekt. In Abhängigkeit von der Höhe je Unternehmen trägt die Ausgleichszulage zur Stabilisierung des Einkommens bei und wirkt sich entsprechend positiv auf die Kapitaldienstfähigkeit aus. Einkommensstabilisierende Transferzahlungen haben insbesondere bei volatilen Märkten (bei schwankenden Preisen unter Unsicherheit) einen positiven Einfluss auf die Liquidität

12 Betriebe, die landwirtschaftliche Flächen (mit förderfähigen Bodennutzungen) in benachteiligten Gebieten bewirtschaften, können aufgrund von Prosperitäts- oder Bagatellgrenzen von der Förderung durch die Ausgleichszulage ausgeschlossen werden. 
und Stabilität des Unternehmens. Die Kreditwürdigkeit des Unternehmens wird dadurch von Banken positiver eingeschätzt. Dieses gilt aber nur, wenn die Zahlungen als "sicher" und damit als regelmäßige Erträge eingestuft werden (BRAND-SAßEN, 2009).

Flächengebundene Subventionen, die nur für einen Teil der landwirtschaftlich genutzten Fläche gewährt werden, verschaffen Betrieben mit Transferzahlungen einen Wettbewerbsvorteil, insbesondere am Bodenmarkt. Bereits HENRICHSMEYER und WITZKE (1994) verweisen auf mögliche Wettbewerbsverzerrungen infolge der Ausgleichszulage, da die Gebietsabgrenzung als eher willkürlich anzusehen ist.

\subsubsection{Agrarumweltmaßnahmen}

Die Inanspruchnahme von Agrarumweltmaßnahmen ist an die Einhaltung von Bewirtschaftungsrestriktionen gebunden, durch die dem teilnehmenden Betrieb (mehr oder weniger hohe) Einkommenseinbußen oder zusätzliche Kosten entstehen können. Inwieweit Agrarumweltzahlungen eher den Charakter einer flächengebundenen Subventions- oder den einer Kompensationszahlung haben, ist von der Höhe der Agrarumweltzahlungen im Verhältnis $\mathrm{zu}$ den betriebsindividuellen Opportunitätskosten der AUM-Teilnahme abhängig.

Die Einhaltung der Bewirtschaftungsauflagen kann, je nach Agrarumweltmaßnahmen, zu einer Erhöhung oder zu einer Verringerung des Einsatzes einzelner Faktoren führen. So ist z. B. eine maximal zulässige Viehbesatzdichte im Rahmen der Grünlandextensivierung durch Flächenaufstockung oder Viehbestandsabstockung zu erreichen. Der Produktionseffekt je Hektar sollte für inputreduzierende Maßnahmen (Ökologischer Landbau, Grünlandextensivierung) in jedem Fall negativ sein.

Die genannten Beispiele zeigen, dass die Wirkung von Agrarumweltmaßnahmen von den Bewirtschaftungsauflagen und den notwendigen Anpassungsreaktionen der teilnehmenden Betriebe abhängt und damit theoretisch unbestimmt ist. Für verschiedene Agrarumweltmaßnahmen lassen sich jedoch zu erwartende Änderungen des Faktoreinsatzes bzw. der Produktion formulieren:

- Maßnahmen mit geringen Bewirtschaftungsauflagen, im Vergleich zur Situation vor der Teilnahme, haben eher den Charakter einer flächengebundenen Subvention. Die Bewirtschaftungsauflagen liegen nah an der ortsüblichen landwirtschaftlichen Praxis (Grünlandextensivierung) oder nah am Stand der Technik (Mulch- und Direktsaatverfahren). Die gewährten Agrarumweltzahlungen übersteigen die tatsächlichen Opportunitätskosten der Programmteilnahme deutlich. Die Agrarumweltzahlungen wirken wie flächengebundene Subvention und führen ceteris paribus zu einer Ausweitung des Bodeneinsatzes (siehe Kapitel 3.1.1). 
- Agrarumweltmaßnahmen mit hohen Bewirtschaftungsauflagen, im Vergleich zur Situation vor der Teilnahme, sind eher mit vergleichsweise hohen Opportunitätskosten verbunden. Diese Kosten werden durch Agrarumweltzahlungen kompensiert. Der betriebliche Faktoreinsatz wird sich entsprechend den Bewirtschaftungsauflagen ändern. Allerdings wird häufig nur ein geringer Anteil der betrieblichen Fläche unter den Auflagen des Vertragsnaturschutzes und vergleichbarer Maßnahmen bewirtschaftet, so dass die gesamtbetrieblichen Effekte im Durchschnitt aller Teilnehmer zu vernachlässigen sein dürften.

Aus Abbildung 2.2 in Kapitel 2.2.2 geht hervor, dass die Agrarumweltförderung in Deutschland flächenmäßig von Maßnahmen mit weniger restriktiven Bewirtschaftungsauflagen (Grünlandextensivierung, vielfältige Fruchtfolge, Winterbegrünung, MDMVerfahren) dominiert wird. Aus diesem Grund ist zu vermuten, dass der mittlere Effekt von Agrarumweltmaßnahmen eher dem Effekt einer flächengebundenen Subvention entspricht. Dass heißt, es ist eine Ausweitung der betrieblichen LF in Teilnehmerbetrieben zu erwarten. Der Produktionseffekt von Agrarumweltmaßnahmen ist davon abhängig, inwieweit die Nutzungsintensität je Hektar - entweder angestoßen durch die einzuhaltenden Bewirtschaftungsauflagen oder durch die Substitution von anderen Faktoren durch Boden - reduziert wird. Eine konstante Nutzungsintensität hat, bei zunehmender Betriebsgröße, (einzelbetrieblich) einen positiven Produktionseffekt zur Folge.

\section{Indirekte Effekte}

Rein kompensatorisch angelegte Agrarumweltzahlungen haben theoretisch keinen Einfluss auf das Einkommen und sollten demzufolge weder einen Versicherungs- noch einen Vermögenseffekt haben. In der Praxis haben Agrarumweltmaßnahmen einen (geringen) positiven Einkommenseffekt (vgl. NieBerg und OfFERMAnN, 2006; Osterburg und StratMANN, 2002), so dass ein Vermögenseffekt zumindest theoretisch möglich. Ein Liquiditätseffekt erscheint aufgrund der geringen zeitlichen Konstanz der angebotenen Agrarumweltmaßnahmen (und deren Förderbedingungen) weniger relevant als im Fall der Ausgleichszulage.

Agrarumweltzahlungen, die eher den Charakter einer flächengebundenen Subvention haben, können den begünstigten Betrieben einen Wettbewerbsvorteil verschaffen. Einzelbeobachtungen aus Hessen belegen, dass Betriebe ohne AUM-Teilnahme in ihrem betrieblichen Wachstum behindert wurden, da Betriebe mit Agrarumweltmaßnahmen zur Zahlung höherer Pachtpreise in der Lage waren (REITER et al. 2003c). Das Argument der Wettbewerbsverzerrung erscheint für Agrarumweltmaßnahmen noch relevanter als für die Ausgleichszulage. Agrarumweltmaßnahmen können in der Regel ohne räumliche Beschränkungen in Anspruch genommen werden. Die förderfähige Fläche kann - anders als bei der Ausgleichszulage - fast uneingeschränkt mit der Flächennachfrage seitens der teilnehmenden Betriebe wachsen. 


\subsection{Einfluss auf das außerlandwirtschaftliche Arbeitsangebot}

Um den Einfluss von Agrarumweltmaßnahmen und der Ausgleichszulage auf den außerbetrieblichen Arbeitseinsatz zu analysieren, sind die betrieblichen Produktionsentscheidungen im Kontext des Einkommens und der Arbeitszeitallokation des landwirtschaftlichen Haushaltes $\mathrm{zu}$ betrachten. In Unternehmens-Haushalts-Modellen (UHM) können Haushaltsentscheidungen bezüglich der Produktion und der Bereitstellung von Arbeit theoretisch konsistent abgebildet werden (NAKAJIMA, 1986; SingH et al., 1986). Zur Analyse des Effektes von Transferzahlungen auf das Arbeitsangebot des Haushaltes wird in der Regel eine reduzierte Form des UHM verwendet (AHEARN et al., 2005; GoOdwIN et al., 2007).

Um den Haushaltsnutzen zu maximieren, ist ein optimales Verhältnis zwischen Haushaltseinkommen und Freizeit zu wählen. Ein hohes Einkommen kann durch einen hohen Arbeitseinsatz innerhalb und außerhalb des landwirtschaftlichen Betriebes erwirtschaftet werden. Mit steigendem Arbeitseinsatz verringert sich die Freizeit. Der Nutzen des Haushaltes nimmt mit steigender Freizeit und steigendem Einkommen zu. Eine optimale Zeitallokation des Unternehmerhaushaltes ist erreicht, wenn das Wertgrenzprodukt der betrieblichen Arbeit, der außerbetrieblichen Arbeit und der Freizeit gleich sind.

Im separablen UHM sind die Produktions- und Konsumentscheidungen des Unternehmerhaushaltes nur von den exogen determinierten Preisen abhängig, da diese die Opportunitätskosten des Faktoreinsatz im Betrieb widerspiegeln (SADOULET und DE JANVRY, 1995b: 145). Die Annahme exogener Preise ermöglicht eine getrennte (separable) Lösung des UHM: Im ersten Schritt wird die Produktionsentscheidung des Unternehmens optimiert, im zweiten Schritt die Arbeitsangebots- und Konsumentscheidung des Haushaltes (HUFFMAN, 1991: 98).

\subsubsection{Ausgleichszulage}

Der Anreiz flächengebundener Subventionen zur Ausweitung der bewirtschafteten Fläche kann eine Erhöhung des betrieblichen Arbeitseinsatzes nach sich ziehen (vgl. Kapitel 3.1.1). Unter der Annahme, dass flächengebundene Subventionen das Wertgrenzprodukt der betrieblichen Arbeit erhöhen, wird außerbetriebliche Arbeit zu Gunsten betrieblicher Arbeit substituiert (Substitutionseffekt). Das betriebliche Arbeitsangebot nimmt zu, und das außerbetriebliche Arbeitsangebot des Haushaltes geht zurück. Der gesamte Arbeitseinsatz des Haushaltes wird hierdurch nicht berührt, da der relevante Lohnsatz unverändert bleibt (WITZKE, 1993). 
Darüber hinaus kann die Ausgleichszulage den außerbetrieblichen Arbeitseinsatz beeinflussen, wenn durch die Zahlungen der Betriebsgewinn und damit das Haushaltseinkommen insgesamt steigen. In diesem Fall ist theoretisch eine Verringerung des gesamten Arbeitseinsatzes des Haushaltes zu erwarten, da die Nachfrage nach Freizeit (normales Gut) zunimmt, wenn das Haushaltseinkommen steigt (Einkommenseffekt) (WITZKE, 1993).

Die dargelegten Überlegungen zum Effekt der Ausgleichszulage auf das außerbetriebliche Arbeitsangebot beruhen auf der Annahme perfekter Arbeitsmärkte. Diese impliziert, dass bereits kleine Veränderungen des innerbetrieblichen Lohnsatzes zu einer Änderung des außerbetrieblichen Arbeitseinsatzes führen müssten. Die Mobilität von Arbeit wird jedoch durch die vorhandenen Transaktionskosten und fehlende außerbetriebliche Erwerbsmöglichkeiten erschwert (GASSON et al., 1995; SCHMITT et al., 1995; SCHULZ-GREVE, 1994). Die durch die Ausgleichszulage induzierte Änderung der Entlohnung von betrieblicher Arbeit ist im Mittel aller Betriebe vermutlich zu gering, um eine messbare Anpassung des außerbetrieblichen Arbeitseinsatzes zu bewirken.

\subsubsection{Agrarumweltmaßnahmen}

Kompensatorisch angelegte Agrarumweltmaßnahmen (ohne positive Einkommenseffekte) können das außerbetriebliche Arbeitsangebot nur als Folge des Substitutionseffektes beeinflussen. Empirische Ergebnisse zeigen, dass die Teilnahme an Agrarumweltmaßnahmen durchaus mit positiven Einkommenswirkungen verbunden sein kann (NIEBERG und Offermann, 2006; Osterburg und Stratmann, 2002) und eine Reduzierung des Arbeitseinsatzes des Haushaltes theoretisch möglich ist. Aufgrund des (im Durchschnitt) geringen Niveaus der bisher beobachteten Einkommenseffekte, der Unvollkommenheit der Arbeitsmärkte und der geringen Konstanz der Agrarumweltförderung sind messbare Änderungen des außerbetrieblichen Arbeitseinsatzes als Folge von Agrarumweltmaßnahmen eher nicht zu erwarten.

\subsection{Entscheidungsmodell zur Programmteilnahme}

Im Gegensatz zu den meisten Transferzahlungen ist die Förderung von Agrarumweltmaßnahmen und der Ausgleichszulage an Bedingungen gebunden, die den Kreis potenzieller Transferempfänger erheblich einschränken. Die Wirkungen, insbesondere von Agrarumweltmaßnahmen, sind davon abhängig, welche Betriebe sich für die Programmteilnahme entscheiden und welche Faktoren diese Entscheidung positiv oder negativ beeinflussen. 
Die „Entweder-Oder“-Entscheidung der Programmteilnahme lässt sich in einem binären Entscheidungsmodell abbilden. Betriebe, die sich für die Teilnahme an Agrarumweltmaßnahmen entscheiden bzw. eine Ausgleichszulage erhalten, müssen bestimmte Bedingungen erfüllen. Im Gegenzug erhalten diese Betriebe einen positiven Betrag aus den Agrarumweltprämien bzw. die Ausgleichszulage (im folgenden Programmzahlungen). Ein Betrieb ohne Programmteilnahme muss diese Bedingungen nicht erfüllen und erhält keine Programmzahlungen. Die Entscheidung für oder gegen die Programmteilnahme hängt vom individuellen Nutzen ab, den der Betrieb aus der (Nicht-)Teilnahme zieht. Der Nutzen eines Betriebes mit Programmteilnahme $\left(u^{1}\right)$ kann beschrieben werden als (vgl. HYNES und GARVEY, 2009)

$$
u^{1}(\pi+d-c ; n, r, z)
$$

und der Nutzen eines Betriebes ohne Programmteilnahme $\left(u^{0}\right)$ als

$$
u^{0}(\pi ; r, z)
$$

wobei $d$ die Höhe der Programmzahlungen je Betrieb, $\pi$ den Gewinn des landwirtschaftlichen Betriebes und $c$ die Opportunitätskosten der Programmteilnahme repräsentieren. Die Opportunitätskosten umfassen den entgangenen Gewinn im Fall einer alternativen Nutzung der Fläche, die Anpassungskosten zur Einhaltung der Bewirtschaftungsauflagen sowie das mit der Programmteilnahme verbundene Risiko (z. B. erhöhte Kontrollwahrscheinlichkeit). Die Vektoren $n, r$ und $z$ repräsentieren programm-, regions- und betriebsspezifische Merkmale, die den Nutzen der (Nicht-)Teilnahme beeinflussen. Die Entscheidungsfunktion hat entsprechend CHAMBERS und FOSTER (1983) die Form

$$
\begin{aligned}
& y=u^{0}(\pi ; r, z)-u^{1}(\pi+d-c ; n, r, z) \text { mit } \\
& y^{*}=\left\{\begin{array}{lll}
0, & \text { wenn } y \geq 0 & \left(u^{0} \geq u^{1}\right) \\
1, & \text { wenn } y<0 & \left(u^{0}<u^{1}\right)
\end{array}\right.
\end{aligned}
$$

Je negativer $y$ ist, desto größer ist der Nutzen der Programmteilnahme für einen Betrieb. Statt des wahren Wertes für $y$ wird nur ein binär skalierter Indikator der Programmteilnahme beobachtet. Ein Betrieb entscheidet sich für die Programmteilnahme $\left(y^{*}=1\right)$, wenn der Nutzen der Teilnahme den der Nichtteilnahme übersteigt. Ein Betrieb nimmt nicht am Programm teil $\left(y^{*}=0\right)$, wenn der betriebsindividuelle Nutzen ohne das Programm größer oder gleich ist als in der Situation mit dem Programm. 
Die Faktoren, die den Nutzen der Teilnahme an Agrarumweltmaßnahmen bzw. an der Ausgleichszulage bestimmen $(n, r, z)$, sind je nach Maßnahme verschieden, da sich beide Programme hinsichtlich ihrer Teilnahmebedingungen unterscheiden.

Die Teilnahme an der Ausgleichszulage ist auf Betriebe beschränkt, die mindestens 3 ha LF in benachteiligten Gebieten bewirtschaften. Die AZ-Teilnahme ist mit keinen zusätzlichen Kosten verbunden, da die Zuwendungsvoraussetzungen ohnehin einzuhalten sind (z. B. Gute Fachliche Praxis) bzw. deutlich oberhalb der ortsüblichen Flächenbewirtschaftung liegen (z. B. 2,0 Großvieheinheiten je Betrieb). Die AZ-Teilnahme wird daher maßgeblich durch die Lage des Betriebes im benachteiligten Gebiet bestimmt. Die Abgrenzung benachteiligter Gebiete erfolgte auf Basis standörtlicher (Höhenlage, Bodengüte), agrarstruktureller (Anteil Grünland) und ökonomischer Faktoren (Abwanderung) (PETERS, 1980), die im Modell zu regionalen Faktoren $(r)$ zusammengefasst sind. Die Entscheidung eines Betriebes für die AZ-Teilnahme ist damit streng genommen keine Selbstselektion, sondern eine durch administrative Kriterien gesteuerte externe Selektion. Alle förderfähigen Betriebe werden die Ausgleichszulage in Anspruch nehmen, da hiermit ein positiver Nutzen verbunden ist.

Die Teilnahme an Agrarumweltmaßnahmen ist ebenfalls freiwillig und an die Einhaltung der Bewirtschaftungsauflagen gebunden. Der Nutzen der Teilnahme ergibt sich aus der Höhe der Agrarumweltzahlungen im Verhältnis zu den entstehenden Opportunitätskosten. Je stärker die aktuelle Flächenbewirtschaftung von den einzuhaltenden Bewirtschaftungsauflagen abweicht, desto höher sind in der Regel die Opportunitätskosten. Für die AUMTeilnahme werden sich nur jene Betriebe entscheiden, deren Opportunitätskosten vollständig durch die Programmzahlungen kompensiert werden. Da sich die Höhe der Agrarumweltzahlungen an den durchschnittlichen Opportunitätskosten orientiert, werden nur Betriebe mit geringen Opportunitätskosten, d. h. mit einem geringen Anpassungsbedarf, an Agrarumweltmaßnahmen teilnehmen.

Die Bestimmungsgründe der Inanspruchnahme von Agrarumweltmaßnahmen werden in der Literatur in programmspezifische $(n)$ und betriebspezifische $(z)$ Faktoren unterteilt (vgl. Brotherton, 1991; Wilson, 1997). Zusätzlich werden in dieser Arbeit regionale Faktoren $(r)$ berücksichtigt, da z. B. die Umsetzung von Agrarumweltmaßnahmen in Deutschland zwischen den Bundesländern (angebotene Maßnahmen, Höhe der Prämiensätze) stark variiert. Zu den programmspezifischen Faktoren zählen die Höhe der Programmzahlungen, die mit der Teilnahme verbundenen Bewirtschaftungsrestriktionen und die Dauer der Programmteilnahme. Die Akzeptanz von Agrarumweltmaßnahmen nimmt mit steigenden Bewirtschaftungsrestriktionen und mit zunehmender Dauer des Verpflichtungszeitraumes ab (VANSELMBROUCK et al., 2002; REITER et al., 2006a, 2006c). 
Unter den betriebsspezifischen Faktoren beeinflussen vor allem jene die Programmteilnahme, die die Höhe der Opportunitätskosten widerspiegeln. Durch Agrarumweltmaßnahmen werden vor allem extensive Bewirtschaftungsformen gefördert. Eine geringe Nutzungsintensität (Viehbesatzdichte, geringer Einsatz von Pflanzenschutz- und Düngemitteln), ein hoher Grünlandanteil und geringe tierische Leistungen bzw. pflanzliche Erträge senken die Opportunitätskosten der Teilnahme und begünstigen die Programmteilnahme (WYNN et al., 2001). Eine niedrige Nutzungsintensität ist häufig auf ungünstige Standortbedingungen (Höhenlage, Bodengüte, kleine Parzellierung) zurückzuführen, weshalb sich die Inanspruchnahme von Agrarumweltmaßnahmen auf benachteiligte Gebiete (CRABTREE et al., 1998) und Regionen mit ungünstigen Standortbedingungen konzentriert (OsterburG et al., 1997).

In vielen Studien wurde auch der Einfluss betriebsleiterspezifischer Merkmale (Alter, Bildung) auf die Teilnahmeentscheidung des Betriebes untersucht (WYNN et al., 2001; VANSLEMBrouck et al. 2002; Defrancesco et al., 2008, Wossink und V. Wenum, 2003). Im Vergleich zu den betriebsspezifischen Faktoren, dürften das Alter und der Bildungsstand des Betriebsleiters eher eine geringere Bedeutung haben.

\subsection{Zusammenfassung}

Der Einfluss von Transferzahlungen auf die Produktion kann in direkte und indirekte Effekte unterteilt werden: Direkte Effekte entstehen als Folge der Änderung von Anreizpreisen oder durch die mengenmäßige Begrenzung der Produktion (Preis- und Substitutionseffekte). Indirekte Effekte auf die Produktion sind aufgrund geänderter (Einkommens)Erwartungen der Produzenten zu erwarten.

Die OECD (2001b) unterscheidet fünf Arten direkter Transferzahlungen: Marktpreisstützungen, Subventionen für Produkte, für variable Inputs und für Boden sowie auf historischen Prämienansprüchen basierende Subventionen. Die Ausgleichszulage und Agrarumweltmaßnahmen haben den Charakter einer flächengebundenen Subvention, deren Erhalt an bestimmte Bedingungen geknüpft ist. Unter der Annahme der (begrenzten) Substituierbarkeit einzelner Produktionsfaktoren wird ein gewinnmaximierender Betrieb den Einsatz eines sich verbilligenden Faktors (Boden) relativ stärker ausdehnen als den Einsatz der übrigen, nicht subventionierten Faktoren. Der Einsatz komplementärer Faktoren (Arbeit, Kapital etc.) sollte absolut betrachtet ebenfalls zunehmen, während die Faktorintensität sinkt. Theoretisch lässt dies eine Verringerung der Erträge erwarten. Produktionsrelevante Effekte von Agrarumweltmaßnahmen und der Ausgleichszulage sind aufgrund der Kopplung der Zahlungen an den aktuellen Bodeneinsatz theoretisch möglich und maßgeblich von den Bedingungen der Inanspruchnahme beider Programme abhängig. 
Die Ausgleichszulage ist eine zu $100 \%$ einkommenswirksame Subvention, wird jedoch nur innerhalb einer definierten Gebietskulisse (benachteiligte Gebiete) gefördert. Da die insgesamt förderfähige Fläche relativ fix ist, sind dem betrieblichen Flächenwachstum infolge der Ausgleichszulage Grenzen gesetzt. Durch die Ausgleichszulage wird ein Anreiz zum Verbleib im Sektor geschaffen.

Die Teilnahme an Agrarumweltprogrammen ist an die Einhaltung (mehr oder weniger) restriktiver Bewirtschaftungsauflagen gebunden. Der Produktionseffekt je Hektar sollte für inputreduzierende Maßnahmen (Ökologischer Landbau, Grünlandextensivierung) in jedem Fall negativ sein. Dies kann, je nach Agrarumweltmaßnahme, zu einer Erhöhung oder zu einer Verringerung des Einsatzes einzelner Faktoren führen und ist damit theoretisch unbestimmt.

Agrarumweltzahlungen kompensieren den durch die Teilnahme entgangenen Gewinn bzw. die zusätzlich entstandenen Kosten. Für die Programmteilnahme werden sich nur Betriebe entscheiden, deren Nutzen der Teilnahme höher ist als die entstehenden Kosten. Das Niveau und der Umfang von Mitnahmeeffekten hat einen Einfluss auf die Produktionseffekte von Agrarumweltmaßnahmen: Maßnahmen mit geringen Bewirtschaftungsauflagen (z. B. Grünlandextensivierung, Mulch- und Direktsaatverfahren) dürften eher den Charakter einer flächengebundenen Subvention haben und einen Anreiz zur Ausweitung der LF schaffen. Zahlungen für Maßnahmen mit hohen Bewirtschaftungsauflagen dienen eher der Kompensation des Mehraufwandes bzw. des Minderertrages. Der Effekt der AUMTeilnahme auf den betrieblichen Faktoreinsatz hängt in diesen Fällen von den Bewirtschaftungsauflagen ab.

Die Ausgleichszulage und Agrarumweltmaßnahmen haben vermutlich einen zu vernachlässigenden Effekt auf den außerbetrieblichen Arbeitseinsatz des Haushaltes. Das Niveau der zu erwartenden Einkommens- und Substitutionseffekte ist insgesamt zu gering, um eine Veränderung der Arbeitszeitallokation des Haushaltes zu bewirken. 


\section{Wirkungen direkter Transferzahlungen auf den Faktoreinsatz, die Produktion und den außerbetrieblichen Arbeitseinsatz - Stand der Forschung}

In diesem Kapitel stehen die Wirkungen direkter Transferzahlungen auf den betrieblichen Faktoreinsatz (Kapitel 4.1 bis 4.4), die Produktion (Kapitel 4.5) und den außerbetrieblichen Arbeitseinsatz landwirtschaftlicher Haushalte (Kapitel 4.3) im Mittelpunkt. Tabelle 4.1 zeigt eine Zusammenstellung empirischer Arbeiten, in denen die Wirkung(en) von geund entkoppelten Direktzahlungen sowie von agrarstruktur- und agrarumweltpolitischen Instrumenten untersucht wird.

Analysen zum Effekt von ge- und entkoppelten Direktzahlungen liegen fast ausschließlich aus den USA vor. Die Politikeffekte werden sowohl auf einzelbetrieblicher (GoODWIN et al., 2007; KEY et al., 2005; SHAIK und HELMERS, 2006) als auch auf regionaler Ebene analysiert (Evenson und Huffman, 1997; Goetz und Debertin, 2001; Kim et al., 2005). HOFER (2002) und RÖSTI (1997) führten vergleichbare Analysen für die Schweiz, GlaubEN et al. (2005) für Deutschland durch. Im Mittelpunkt dieser Studien stehen die Wirkungen von Direktzahlungen auf die Agrarstruktur (Anzahl bzw. Fortbestand von Betrieben), den betrieblichen Faktoreinsatz (Boden, Arbeit etc.) sowie auf die Arbeitsallokation zwischen betrieblichen und außerbetrieblichen Tätigkeiten.

In Europa wurden vor allem empirische Untersuchungen zu den Wirkungen struktur- und umweltpolitischer Programme, z. B. für Agrarumweltmaßnahmen (NIEBERG et al., 2005; Osterburg, 2005; Pufahl und Weiss, 2009), die Ausgleichszulage für benachteiligte Gebiete (IEEP, 2006; PLANKL et al., 2005), die Vorruhestandsregelung für Landwirte (PIETOla et al., 2003) und das Agrarinvestitionsprogramm (BERGSCHMIDT et al., 2006; BRÜMMER und LOY, 2000) durchgeführt. Die Wirkungsanalyse dieser Maßnahmen erfolgt fast ausschließlich einzelbetrieblich und vor dem Hintergrund maßnahmenspezifischer Zielsetzungen (z. B. Umwelt, Einkommen).

\subsection{Betriebsaufgabe und Anzahl von Betrieben}

Aus den 80er- und 90er-Jahren liegen zahlreiche Untersuchungen zu den Bestimmungsgründen des Ausstiegs bzw. des Rückgangs der Anzahl landwirtschaftlicher Betriebe vor (BAur, 1997; Hallam, 1993; Sumner und Leiby, 1987; Weiss, 1999). Die wesentlichen Erkenntnisse hieraus sind, dass ausscheidende Betriebe in der Regel eine unterdurchschnittliche Betriebsgröße (LF, Viehbesatz) aufweisen. Der Zeitpunkt der Betriebsaufgabe fällt, bei fehlendem Hofnachfolger, meist mit dem Renteneintritt des Betriebsleiters zusammen. 
Tabelle 4.1: $\quad$ Literaturüberblick - Wirkungen direkter Transferzahlungen auf den betrieblichen Faktoreinsatz, die Produktion und den außerbetrieblichen Arbeitseinsatz

\begin{tabular}{|c|c|c|c|c|c|c|c|c|}
\hline Autor(en) & $\begin{array}{l}\text { Art der direkten } \\
\text { Transferzahlung }\end{array}$ & $\begin{array}{l}\text { Unter- } \\
\text { suchungs- } \\
\text { ebene, } \\
\text { Land }\end{array}$ & 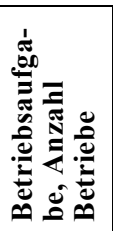 & 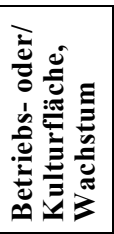 & 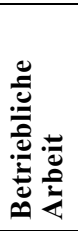 & 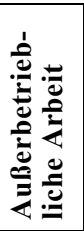 & 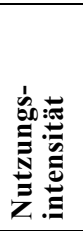 & 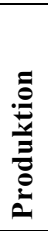 \\
\hline KEY \& ROBERTS (2006) & DZ* & E, US & - & & & & & \\
\hline GOETZ \& DEBERTIN (2001) & DZ* & A, US & + & & & & & \\
\hline GLAUBEN et al. (2005) & $\mathrm{DZ} *$ & $\mathrm{~A}, \mathrm{D}$ & 0 & & & & & \\
\hline KIM et al. (2005) & DZ* & $\mathrm{A}, \mathrm{US}$ & $+/-$ & & & & & \\
\hline PIETOLA et al. (2003) & Gekoppelte DZ & E, FIN & 0 & & & & & \\
\hline SINABELL \& STREICHER (2005) & $\begin{array}{l}\text { Struktur (AFP) } \\
\text { Umwelt (Öko) }\end{array}$ & $\mathrm{E}, \mathrm{AUT}$ & $\begin{array}{l}+ \\
0\end{array}$ & & & & & \\
\hline RÖSTI (1997) & Entkoppelte DZ & $\mathrm{A}, \mathrm{CH}$ & + & & & & & \\
\hline KEY \& ROBERTS (2003) & DZ* & E, US & - & $+/ 0$ & & & & \\
\hline AHEARN et al. (2002) & $\begin{array}{l}\text { Gekoppelte DZ } \\
\text { Umwelt (CRP) }\end{array}$ & A, US & + & + & & $\overline{0}$ & & \\
\hline MANN (2003) & Entkoppelte DZ & $\mathrm{A}, \mathrm{CH}$ & + & & 0 & & & \\
\hline WEISS (2006b; 2006a) & $\begin{array}{l}\text { Umwelt (AUM) } \\
\text { Struktur (AZ) } \\
\text { DZ, AUM, AZ }\end{array}$ & $\mathrm{E}, \mathrm{AUT}$ & $\begin{array}{l}- \\
0 \\
-\end{array}$ & & + & & & \\
\hline HOFER (2002) & Entkoppelte DZ & $\mathrm{E}, \mathrm{CH}$ & - & & & - & & \\
\hline AHEARN et al. (2005) & $\begin{array}{l}\text { Gekoppelte DZ } \\
\text { Umwelt (CRP) }\end{array}$ & A, US & $\begin{array}{l}0 /+ \\
-/+\end{array}$ & & & $\begin{array}{l}0 \\
0\end{array}$ & & \\
\hline SHAIK \& HELMERS (2006) & $\mathrm{DZ} *$ & A, US & & + & 0 & & & \\
\hline LAMBERT \& GRIFFIN (2004) & DZ* & E, US & & + & + & & - & \\
\hline BECK \& DOGOT (2006) & Struktur(AFP) & E, BEL & & + & + & & & \\
\hline PFEFFERLI (2006) & Struktur (AFP) & $\mathrm{E}, \mathrm{CH}$ & & + & - & & & \\
\hline PUFAHL \& WEISS (2009) & $\begin{array}{l}\text { Umwelt (AUM) } \\
\text { Struktur (AZ) }\end{array}$ & E, D & & $\begin{array}{l}+ \\
+ \\
+\end{array}$ & $\begin{array}{l}+ \\
0\end{array}$ & $\begin{array}{l}0 \\
0\end{array}$ & $\overline{0}$ & $\begin{array}{l}++ \\
+\end{array}$ \\
\hline GOODWIN et al. (2007) & $\begin{array}{l}\text { Entkoppelte DZ } \\
\text { Gekoppelte DZ } \\
\text { Sonstige DZ } \\
\text { Umwelt (CRP) } \\
\end{array}$ & E, US & & $\begin{array}{l}- \\
+ \\
+\end{array}$ & & $\begin{array}{l}0 /- \\
0 /+ \\
0 / 0 \\
0 / 0\end{array}$ & & \\
\hline HUFFMAN \& EVANSON (2001) & Gekoppelte DZ & A, US & & + & & - & & \\
\hline KEY et al. (2005) & DZ* & E, US & & + & & & & + \\
\hline $\begin{array}{l}\text { OSTERBURG \& STRATMANN (2002), } \\
\text { OSTERBURG (2005) }\end{array}$ & $\begin{array}{l}\text { Umwelt (AUM) } \\
\text { Umwelt (Öko) }\end{array}$ & E, D & & $\begin{array}{l}+ \\
+ \\
+\end{array}$ & & & $\begin{array}{l}- \\
-\end{array}$ & \\
\hline EL-OSTA et al. (2004) & $\begin{array}{l}\text { Entkoppelte DZ } \\
\text { Gekoppelte DZ } \\
\text { Sonstige }\end{array}$ & $\mathrm{E}, \mathrm{US}$ & & & $\begin{array}{l}+ \\
0 \\
+\end{array}$ & $\begin{array}{l}- \\
- \\
0\end{array}$ & & \\
\hline OFFERMANN \& NIEBERG $(2001 ; 2003)$ & Umwelt (Öko) & E, EU, D & & & + & & & \\
\hline $\begin{array}{l}\text { NIEBERG (1996), NIEBERG \& OFFERMANN } \\
(2006)\end{array}$ & Umwelt (Öko) & E, D & & & + & & - & - \\
\hline CHANG \& BOISVERT (2005) & DZ* & E, US & & & & - & & \\
\hline GOODWIN \& MISHRA (2004) & Entkoppelte DZ & E, US & & & & - & & \\
\hline MISHRA \& GOODWIN (1997) & Entkoppelte DZ & E, US & & & & $-/-$ & & \\
\hline SERRA et al. (2005) & $\begin{array}{l}\text { Entkoppelte DZ } \\
\text { Gekoppelte DZ }\end{array}$ & $\mathrm{E}, \mathrm{US}$ & & & & $\overline{0}$ & & \\
\hline AHEARN et al. (2006) & $\begin{array}{l}\text { Entkoppelte DZ } \\
\text { Gekoppelte DZ } \\
\text { Umwelt (CRP) }\end{array}$ & $\mathrm{E}, \mathrm{US}$ & & & & $\begin{array}{l}-/- \\
-/ 0 \\
0 /-\end{array}$ & & \\
\hline ADAMS et al. (2001) & Entkoppelte DZ & $\mathrm{A}, \mathrm{US}$ & & & & & & + \\
\hline GIRANTE et al. (2008), ROBERTS (2008) & Entkoppelte DZ & E, US & & & & & & + \\
\hline SALHOFER \& STREICHER (2005) & $\begin{array}{l}\text { Umwelt (AUM) } \\
\text { Umwelt (Öko) }\end{array}$ & $\mathrm{E}, \mathrm{AUT}$ & & & & & & $\begin{array}{ll}0 \\
-\end{array}$ \\
\hline MORO \& SCKOKAI (1999) & Gekoppelte DZ & E, ITA & & + & & & $+/-/ 0$ & + \\
\hline
\end{tabular}

$\mathrm{DZ}=$ Direktzahlungen, Struktur $=$ Strukturprogramme, Umwelt $=$ Umweltprogramme, $\mathrm{E}=$ Einzelbetriebliche Analyse, $\mathrm{A}=$ Aggregierte Analyse (Nuts I/II/III), CRP = Conservation Reserve Programme, AFP = Agrarinvestitionsförderung, AUM = Agrarumweltmaßnahmen (ohne Öko), Öko = AUM Ökologischer Landbau, AZ = Ausgleichszulage für benachteiligte Gebiete. $0=$ keine Wirkung, + = positive Wirkung, - = negative Wirkung, / = Ergebnisse für verschiedene Betriebs-/Personengruppen, ${ }^{*}$ ohne Differenzierung zwischen ge- und entkoppelten Direktzahlungen.

Quelle: eigene Zusammenstellung nach angegebenen Quellen. Ausführlich in Tabelle A1. 
Erst mit zunehmender Datenverfügbarkeit konnte auch der Einfluss von direkten Transferzahlungen auf die Betriebsaufgabe analysiert werden: Arbeiten auf einzelbetrieblicher Ebene liegen für die USA von KEY und RoBERTS (2006; 2003) und GoEtZ und DeBERTIN (2001), für die Schweiz von Hofer (2002), für Deutschland von Glauben et al. (2005) und von Pietola et al. (2003) für Finnland vor. Der Einfluss von direkten Transferzahlungen auf die Anzahl der landwirtschaftlichen Betriebe wurde von AHEARN et al. (2005) und KIM et al. (2005) für die USA sowie von RÖSTI (1997) und MANN (2003) für die Schweiz untersucht.

Die Arbeiten zeigen fast ausnahmslos eine strukturkonservierende Wirkung von (ge- und entkoppelten) Direktzahlungen und umweltorientierten Zahlungen: je höher die Transferzahlungen, desto geringer ist die Ausstiegswahrscheinlichkeit bzw. desto höher die Wahrscheinlichkeit des Fortbestandes von Betrieben. Mit zunehmender Höhe der Direktzahlungen schwächt sich deren strukturhemmende Wirkung ab (HOFER, 2002).

Goetz und DeBertin (2001) zeigen, dass die Wirkung von Direktzahlungen auf den Erhalt landwirtschaftlicher Betriebe zwischen Regionen variieren kann: Bei der Betrachtung aller US-Bundesstaaten reduzieren Direktzahlungen die Ausstiegsrate von Betrieben. Eine den Ausstieg von Betrieben beschleunigende Wirkung haben Direktzahlungen in den Bundesstaaten, in denen die Anzahl landwirtschaftlicher Betriebe insgesamt zurückgeht. Goetz und Debertin begründen dies damit, dass die im Sektor verbleibenden Betriebe durch die Direktzahlungen in die Lage versetzt werden, die ausstiegswilligen Betriebe aufzukaufen (vgl. Key und RoBerts, 2006). Die Ergebnisse von GoEtz und DebertiN unterstreichen die Bedeutung der regionalen Bedingungen für den Verlauf des Strukturwandels. Wechselwirkungen zwischen landwirtschaftlichen Betrieben bestehen über den Bodenmarkt. Demzufolge dürften sich auch die Wirkungen von Transferzahlungen auf den landwirtschaftlichen Strukturwandel zwischen Regionen bzw. Betrieben unterscheiden.

Pietola et al. (2003) untersuchen nur Betriebe, deren Leiter aus Altersgründen vor der Entscheidung stehen, den Betrieb aufzugeben oder diesen an einen Nachfolger zu übergeben. Während kein Einfluss von gekoppelten Direktzahlungen auf die Betriebsaufgabe zu beobachten ist, steigt die Wahrscheinlichkeit der Betriebsübernahme durch einen Nachfolger mit steigender Höhe gekoppelter Direktzahlungen.

Welche Betriebsgruppen (Größenklassen, Betriebsformen) von der strukturkonservierenden Wirkung direkter Transferzahlungen überproportional profitieren, scheint noch offen zu sein. KIM et al. (2005) zeigen, dass (ge- und entkoppelte) Direktzahlungen die Anzahl kleiner Betriebe erhöhen und die Anzahl großer Betriebe verringern. Als Profiteure der 
strukturkonservierenden Wirkung werden also hauptsächlich kleine Betriebe ${ }^{13}$ identifiziert: Direktzahlungen haben in kleinen Betrieben häufig einen hohen Anteil am Betriebseinkommen und sind für den Fortbestand des Betriebes bedeutsam. KEY und RoBERTS (2006) kommen zu einem gegenteiligen Ergebnis. Die Autoren zeigen, dass der positive Einfluss von (ge- und entkoppelten) Direktzahlungen auf den Fortbestand von Betrieben mit steigender Betriebsgröße zunimmt, also große Betriebe überproportional vom verminderten Ausstiegsrisiko profitieren. Allerdings berücksichtigen KEY und ROBERTS (2006) in ihrer Analyse ausschließlich Betriebe mit Direktzahlungen. Die Ergebnisse könnten durch den Ausschluss von großen Betrieben ohne Direktzahlungen verzerrt sein.

Zum Einfluss umweltorientierter Programme auf die Ausstiegswahrscheinlichkeit von landwirtschaftlichen Betrieben liegen zwei Untersuchungen vor. Die Teilnahme an Agrarumweltprogrammen in Österreich ist mit einer reduzierten Wahrscheinlichkeit der Betriebsaufgabe verbunden, während für die Ausgleichszulage kein Einfluss auf die Ausstiegsentscheidung zu beobachten ist (WEISS, 2006a). Diese Ergebnisse sind jedoch nicht auf Deutschland übertragbar, da die je Hektar gewährten Agrarumweltzahlungen in Österreich zu den höchsten in Europa zählen und deutlich über dem deutschen Niveau liegen (Hovorka, 2006). AHEARN et al. (2005) beobachten für die USA ebenfalls ein reduziertes Ausstiegsrisiko für Betriebe mit Teilnahme am Conservation Reserve Program.

\subsection{Betriebliche Flächenausstattung und Flächenwachstum}

Zum Einfluss von direkten Transferzahlungen auf die landwirtschaftlich genutzte Fläche eines Betriebes bzw. den Anbauumfang bestimmter Kulturen liegen zahlreiche Untersuchungen auf einzelbetrieblicher (KEY et al., 2005; LAMBERT und GRIFFIN, 2004; Osterburg und Stratmann, 2002; Pufahl und Weiss, 2009) und aggregierter Ebene (AHEARn et al., 2002; SHAik und HELMERS, 2006) vor. Die in Tabelle 4.1 aufgeführten Arbeiten weisen fast ausnahmslos einen positiven Zusammenhang zwischen direkten Transferzahlungen und der betrieblichen Flächenausstattung (insgesamt und kulturartenspezifisch) bzw. dem betrieblichen Flächenwachstum aus.

GoODWIN et al. (2007) beobachten einen negativen Zusammenhang zwischen entkoppelten Direktzahlungen und der betrieblichen Flächenausstattung. Sie begründen ihre Beobachtung mit dem Gewinneffekt entkoppelter Direktzahlungen: Landwirtschaftliche Arbeit und der Umfang der bewirtschafteten Fläche wird zu Gunsten von Freizeit reduziert. Für gekoppelte Zahlungen stellen GoODWIN et al. (2007) einen signifikant positiven Einfluss auf

13 Zu den kleinen Betrieben zählen etwa $80 \%$ der US-Betriebe (Nebenerwerbsbetriebe mit negativem Betriebseinkommen). 
die betriebliche LF fest, da durch Flächenwachstum der Gewinn des Betriebes (und die Summe der Direktzahlungen) erhöht werden kann.

KEY und RoBerts (2003) analysieren US-amerikanische Ackerbaubetriebe mit Direktzahlungen, differenziert nach Hauptanbaukulturen und Betriebsgrößenklassen. Ein systematischer Zusammenhang zwischen der Betriebsgröße und der Höhe von Direktzahlungen ist nicht zu beobachten. Betriebe mit Mais- und Marktfruchtanbau dehnen unter dem Einfluss von Direktzahlungen die Anbaufläche für diese Kulturen signifikant aus, während für andere Produktionsrichtungen (Soja, Weizen, Baumwolle und Reis) kein solcher Effekt festzustellen ist.

HUFFMAN und EVENSON (2001) untersuchen neben anderen agrarpolitischen Maßnahmen ${ }^{14}$ den Effekt von gekoppelten Direktzahlungen auf die betriebliche Flächenausstattung. Für den Zeitraum 1953 bis 1982 beobachten die Autoren eine signifikante Erhöhung der betrieblichen LF als Folge gekoppelter Direktzahlungen. Öffentliche Ausgaben für Beratungsmaßnahmen wirkten positiv auf die Betriebsgröße.

Der empirische Befund zur Wirkung agrarstruktur- und umweltpolitischer Instrumente auf die betriebliche Flächenausstattung deutet ebenfalls auf einen positiven Zusammenhang hin: Ergebnisse von Osterburg und Stratmann (2002) sowie Pufahl und Weiss (2009) zeigen, dass Betriebe mit Teilnahme an Agrarumweltmaßnahmen oder der Ausgleichszulage ein deutlich höheres Flächenwachstum, insbesondere von Grünland, aufweisen als Betriebe ohne Programmteilnahme. Das Ausmaß des programminduzierten Flächenwachstums ist unter anderem abhängig von der Art der Agrarumweltmaßnahme (Ökologischer Landbau und andere), vom Bundesland (Osterburg, 2005) und der Dauer der Programmteilnahme (PUFAHL und WEISS, 2007).

Die Agrarinvestitionsförderung (AFP) könnte ebenfalls einen positiven Einfluss auf das betriebliche Flächenwachstum haben, da primär Wachstumsinvestitionen in landwirtschaftliche Betriebe bezuschusst werden (BECK und DogOT, 2006; PfEFfERLI, 2006). Grundsätzlich ist die Kausalität zwischen der AFP-Förderung und den beobachteten Veränderungen des betrieblichen Faktoreinsatzes zu hinterfragen. Vermutlich ist die beobachtete Veränderung primär auf die Investition selbst und nicht auf den Investitionszuschuss zurückzuführen, da in der Praxis nahezu jeder Betrieb gefördert wird, der eine AFPFörderung beantragt. 


\subsection{Betriebliches und außerbetriebliches Arbeitsangebot}

Die empirischen Ergebnisse zum Einfluss von direkten Transferzahlungen auf die Zeitallokation zwischen betrieblicher Tätigkeit und außerbetrieblicher Erwerbsarbeit ergeben ein sehr heterogenes Bild.

\section{Betrieblicher Arbeitseinsatz}

Der Einfluss von direkten Transferzahlungen auf den betrieblichen Arbeitseinsatz scheint in der Tendenz neutral bis positiv zu sein (vgl. Tabelle 4.1). Dies gilt gleichermaßen für ge- und entkoppelte Direktzahlungen sowie für agrarstruktur- und agrarumweltpolitische Programme.

Den Einfluss von Direktzahlungen auf den Arbeitseinsatz in landwirtschaftlichen Betrieben untersuchen MANN (2003) für die Schweiz (entkoppelte Direktzahlungen) sowie SHAIK und Helmers (2006) für Nebraska, USA (ge- und entkoppelte Direktzahlungen). Beide Studien weisen einen neutralen Effekt von Direktzahlungen auf die Anzahl der betrieblichen Arbeitskrafteinheiten aus.

Einzelbetriebliche Analysen zum Zusammenhang zwischen Direktzahlungen und dem betrieblichen Arbeitseinsatz liegen für die USA von LAMBERT und GRIFFIN (2004) sowie von EL-OstA et al. (2004) vor. LAMBERT und GRIFFIN (2004) differenzieren nicht zwischen geund entkoppelten Direktzahlungen, die insgesamt einen positiven Einfluss auf die Anzahl der betrieblichen Arbeitskrafteinheiten haben. EL-OsTA et al. (2004) untersuchen das betriebliche Arbeitsangebot des Betriebsleiters in Abhängigkeit von gekoppelten, entkoppelten sowie sonstigen Direktzahlungen. Für gekoppelte Direktzahlungen ist kein Einfluss auf den betrieblichen Arbeitseinsatz des Betriebsleiters zu beobachten. Entkoppelte und sonstige Direktzahlungen erhöhen dagegen die betriebliche Arbeitszeit des Betriebsleiters statistisch signifikant, allerdings nur in einem ökonomisch wenig bedeutsamen Umfang. Dieses Ergebnis ist zunächst überraschend, da für gekoppelte Direktzahlungen theoretisch ein positiver Effekt und für entkoppelte Direktzahlungen ein neutraler Effekt auf den betrieblichen Arbeitseinsatz zu erwarten gewesen wäre. EL-OsTA et al. (2004) folgern hieraus, dass Direktzahlungen dem allgemeinen Trend einer zunehmenden außerlandwirtschaftlichen Erwerbsbeteiligung von Haushaltsmitgliedern entgegengewirkt haben, diesen aber nicht umkehren konnten.

Der im Rahmen von Agrarumweltmaßnahmen geförderte ökologische Landbau hat im bundesdeutschen Durchschnitt einen positiven Einfluss auf den betrieblichen Arbeitskrafteinsatz (Offermann und Nieberg, 2001). Pufahl und Weiss (2009) zeigen für Deutschland, dass die Teilnahme an Agrarumweltmaßnahmen (den ökologischen Landbau ausgenommen) einen ökonomisch geringen, aber statistisch signifikant positiven Effekt auf den 
betrieblichen Arbeitseinsatz hat. Für die Ausgleichszulage ist kein vergleichbarer Effekt zu beobachten (vgl. auch BURGATH et al., 2001; IEEP, 2006).

WEISS (2006b) untersucht für Österreich den Einfluss von Agrarumweltmaßnahmen, der Ausgleichszulage und nationalen Beihilfen auf die Veränderung des Erwerbscharakters des landwirtschaftlichen Betriebes. ${ }^{15}$ Mit steigenden Programmzahlungen verringert sich die Wahrscheinlichkeit des Übergangs vom Haupt- in den Nebenerwerb bzw. die Wahrscheinlichkeit, dass der betriebliche Arbeitseinsatz zu Gunsten der außerbetrieblichen Erwerbsbeteiligung reduziert wird. Die Programmzahlungen haben, bei gleicher Höhe, in kleinen Betrieben eine höhere beschäftigungserhaltende Wirkung als in großen Betrieben.

Einen kausalen Zusammenhang zwischen der AFP-Förderung und dem betrieblichen Arbeitseinsatz vorausgesetzt, ist die Richtung des Beschäftigungseffektes primär von der Art der Investitionen abhängig: Rationalisierungsinvestitionen führen eher zu negativen $\mathrm{Be}-$ schäftigungseffekten (vgl. PfEFfERLI, 2006, für die Schweiz). Werden schwerpunktmäßig Wachstumsinvestitionen gefördert, so sind einzelbetrieblich auch positive Beschäftigungseffekte möglich (vgl. BECK und DOGOT, 2006, für Belgien).

\section{Außerbetriebliches Arbeitsangebot}

Die meisten empirischen Studien analysieren den Zusammenhang zwischen direkten Transferzahlungen und der außerbetrieblichen Erwerbsbeteiligung, der eher aus der Sicht nationaler Politik von Relevanz ist (AHEARN et al., 2006). Das außerbetriebliche Arbeitsangebot wird in den untersuchten Studien durch die Partizipationsentscheidung des Betriebsleiters bzw. der Partnerin am Arbeitsmarkt (AHEARn et al., 2006; Hofer, 2002; HuFFMAN und EVENSON, 2001) oder durch den Umfang der außerbetrieblichen Erwerbsarbeit (El-Osta et al., 2004; Goodwin und Mishra, 2004; Mishra und Goodwin, 1997) abgebildet.

Die empirischen Ergebnisse in Tabelle 4.1 zeigen in der Tendenz einen negativen Einfluss ge- und entkoppelter Direktzahlungen auf das außerbetriebliche Arbeitsangebot (AHEARN et al., 2006; El-Osta et al., 2004; GoODWIN und Mishra, 2004; HofER, 2002; HufFMAN und Evenson, 2001). Lediglich Goodwin et al. (2007) beobachten einen positiven Einfluss gekoppelter Direktzahlungen auf den Umfang des außerbetrieblichen Arbeitsangebotes der Partnerin (vgl. auch EL-OstA et al., 2008).

15 Der Erwerbscharakter landwirtschaftlicher Betriebe in Österreich wird auf Basis der Zeitallokation zwischen betrieblicher und außerbetrieblicher Arbeit bestimmt. Der Übergang vom Haupt- zum Nebenerwerb geht daher mit der Reduktion betrieblicher zu Gunsten außerbetrieblicher Arbeit einher. 
$\mathrm{Ob}$ die durch Direktzahlungen induzierte Verringerung der außerbetrieblichen Erwerbsarbeit eine Folge des Gewinn- oder des Substitutionseffektes ist, kann nur beurteilt werden, wenn Informationen zum betrieblichen und außerbetrieblichen Arbeitseinsatz vorliegen. Nur drei der in Tabelle 4.1 aufgeführten Studien betrachten beide Aspekte gleichzeitig (El-Osta et al., 2004; Pufahl und Weiss, 2009; Shaik und Helmers, 2006).

Einen Gewinneffekt beobachten SHAIK und Helmers (2006) für Direktzahlungen allgemein sowie EL-OstA et al. (2004) für gekoppelte Direktzahlungen: Außerbetriebliche Erwerbsarbeit wird, bei gleich bleibendem betrieblichen Arbeitseinsatz, zu Gunsten von Freizeit reduziert. Entgegen den theoretischen Erwartungen ist eine Substitution von außerbetrieblicher durch betriebliche Arbeit bisher nur für entkoppelte Direktzahlungen belegt (EL-OstA et al., 2004).

Umwelt- und strukturpolitische Programme scheinen in der Tendenz keinen Einfluss auf die außerbetriebliche Erwerbsbeteiligung des Betriebsleiter(paare)s zu haben (AHEARN et al., 2005; GooDwIN et al., 2007). Dies kann darauf zurückzuführen sein, dass die Höhe der Programmzahlungen verhältnismäßig gering ist.

\subsection{Umweltrelevante Änderungen des Faktoreinsatzes}

Der umweltrelevante Faktoreinsatz bezieht sich auf die Nutzungsintensität der LF (Viehbesatzdichte, Pflanzenschutz- und Düngemittelaufwand je Hektar LF). Die Änderung des umweltrelevanten Faktoreinsatzes ist primär für Programme mit umweltrelevanten Zielen von Bedeutung und folgt zumeist direkt aus den Bedingungen der Programmteilnahme. ${ }^{16}$ Eine Verringerung der Nutzungsintensität infolge ge- und entkoppelter Direktzahlungen ist nicht zu beobachten (LAMBERT et al., 2006) bzw. wird aufgrund der fehlenden Zielsetzung nur selten untersucht.

Infolge der Teilnahme an Agrarumweltmaßnahmen ist eine statistisch signifikante Verringerung der Nutzungsintensität der bewirtschaften Fläche zu beobachten (OSTERBURG und Stratmann, 2002; Pufahl und Weiss, 2009). Gleichzeitig steigt in Teilnehmerbetrieben, im Vergleich zu den Nichtteilnehmern, der Anteil extensiver Bodennutzungen (z. B. Grünland) bzw. verringert sich der Anteil intensiver Kulturen (z. B. Mais) (OsterbuRG, 2005; LAMBERT et al., 2006). Die im Mittel aller teilnehmenden Betriebe vorhandenen positiven Wirkungen von Agrarumweltmaßnahmen müssen allerdings relativiert werden: So zeigen

16 Zur Beurteilung der ökologischen Wirksamkeit von Agrarumweltmaßnahmen ist, neben der Veränderung bzw. Erhaltung einer bestimmten Nutzungsintensität, vor allem die Treffsicherheit von Agrarumweltmaßnahmen in Zielgebieten (Gebiete mit hoher Schutzwürdigkeit oder Schutzbedürftigkeit) relevant. Diese Fragestellung wird hier nicht betrachtet. 
REITER et al. (2006a), dass Agrarumweltmaßnahmen häufig auf bereits extensiv bewirtschafteten Teilflächen, insbesondere auf Grünland, angewendet werden. Die hierdurch induzierte Änderung der Nutzungsintensität ist in vielen Fällen gering bzw. geht gegen Null.

Da die Zahlung der Ausgleichszulage in Deutschland an keine limitierenden Bewirtschaftungsauflagen gebunden ist, sind auch keine über die „Gute fachliche Praxis“ hinausgehenden positiven Umweltwirkungen zu erwarten (PLANKL et al., 2005). Ein zentrales Ziel der Ausgleichszulage ist der Erhalt der landwirtschaftlichen Nutzung (vgl. Kapitel 2.3.1). Das befürchtete großflächige Brachfallen landwirtschaftlich genutzter Flächen in benachteiligten Gebieten ist in Deutschland und in anderen Mitgliedsstaaten der Europäischen Union nicht eingetreten. Die LF in benachteiligten Gebieten ist seit 1990 in etwa konstant geblieben, sieht man von den eher marginalen Änderungen ab, die auf nichtlandwirtschaftliche Faktoren (z. B. Siedlungserweiterungen) zurückzuführen sind (IEEP, 2006: 170).

Die Bedeutung der Ausgleichszulage zum Erhalt der Flächennutzung wird meist aus dem Anteil der Ausgleichszulage am landwirtschaftlichen Einkommen abgeleitet: je höher der Anteil der Ausgleichszulage am betrieblichen Einkommen, desto größer die Bedeutung für den Erhalt der landwirtschaftlichen Flächennutzung (IEEP, 2006: 178). Es wird unterstellt, dass Betriebe ohne die Ausgleichszulage die Bewirtschaftung eingestellt hätten und die Flächen aus Mangel an potenziellen Bewirtschaftern brachgefallen wären. Der empirische Befund für diesen Zusammenhang (Einstellung der Ausgleichszulage $\rightarrow$ Ausstieg von Betrieben $\rightarrow$ Brachfallen von LF) steht noch aus. WEISS (2006a) zeigt für Österreich, dass die Ausgleichszulage keinen Einfluss auf den Fortbestand landwirtschaftlicher Betriebe hat und damit bereits das erste Glied der aufgezeigten Wirkungskette hinfällig ist.

\subsection{Produktionseffekte}

Für die internationale Handelsdebatte sind vor allem die Produktionseffekte von Transferzahlungen von Interesse, da hierdurch das Volumen der am Weltmarkt gehandelten Güter beeinflusst wird. Direkte Transferzahlungen beeinflussen das Produktionsvolumen durch die Veränderung des betrieblichen Faktoreinsatzes (vgl. Kapitel 4.1 bis 4.4) sowie durch verschiedene indirekte Wirkungsmechanismen. Während die einzelnen Wirkungsmechanismen theoretisch und empirisch gut belegt sind, stellt die vergleichbare Quantifizierung von Produktionseffekten verschiedener Arten von Direktzahlungen sowie deren Differenzierung in direkte und indirekte Effekte gegenwärtig noch eine Herausforderung dar. 
An das Produktionsvolumen bzw. den betrieblichen Faktoreinsatz gekoppelte Direktzahlungen schaffen Anreize zur Steigerung der Produktion (OECD, 2001b; WestscotT und Price, 2001, Moro und ScKoKAI, 1999). Das Niveau des Produktionseffektes variiert in Abhängigkeit von der absoluten Höhe und der Art der Subvention (OECD, 2006). Die OECD (2001b) zeigt in einer Simulationsstudie, dass flächengebundene Subventionen den geringsten Einfluss auf die Produktion haben, gefolgt von Marktpreisstützungen und Produktsubventionen. Die mit Abstand höchsten Produktionseffekte haben Subventionen für variable Inputs. Die Ergebnisse dieser Simulationsstudie lassen sich jedoch nur teilweise durch empirische ex-post Analysen bestätigen (vgl. OECD, 2006).

Die Produktionseffekte von entkoppelten Direktzahlungen sind ebenfalls positiv, aber deutlich geringer als die von gekoppelten Direktzahlungen. Dies belegen die Ergebnisse der OECD (2001b) sowie die ökonometrischen Analysen von ADAMS et al. (2001), GIRANTE et al. (2008) und RoBERTs (2008). Im Gegensatz zu gekoppelten Direktzahlungen beeinflussen entkoppelte Direktzahlungen die Produktion eher über indirekte Wirkungsmechanismen.

Ein besonderes Interesse gilt dabei dem Versicherungs- und dem Vermögenseffekt, deren Niveau von der Risikoaversion der Produzenten abhängt (vgl. Kapitel 3.2.1). Der Anteil des Versicherungs- und Vermögenseffektes am Gesamteffekt variiert in Abhängigkeit von der Art der Direktzahlungen. Maßnahmen, die die Variabilität des Einkommens reduzieren, haben vor allem einen Versicherungseffekt. Der Vermögenseffekt dominiert, wenn die Politik besonders effizient Einkommen an landwirtschaftliche Betriebe transferiert (CAhill, 2004). Hennessy (1998) und Mullen et al. (2001, zitiert in CAhill, 2004) zeigen, dass der Gesamteffekt von US deficiency payments durch den Versicherungseffekt dominiert wird, da die Höhe dieser Zahlungen vom aktuellen Preisniveau abhängt.

Betriebe mit einem schweren bzw. unzureichenden Zugang zum Kapitalmarkt erhalten durch entkoppelte Transferzahlungen finanzielle Mittel, um Investitionen durchzuführen. Untersuchungen aus den USA zeigen, dass dieser Effekt bisher nicht empirisch nachzuweisen ist (BURFISHER und Hopkins, 2003). Eine Ursache für das Fehlen dieses theoretisch möglichen Effektes könnte das weitgehende Funktionieren des Kapitalmarktes in entwickelten Volkswirtschaften sein. In einer Situation mit ineffizienten Kapitalmärkten und einem unzureichenden Zugang zu Krediten würden die entkoppelten Direktzahlungen vermutlich zu einer Erhöhung der Investitionen in den landwirtschaftlichen Betrieb führen (ebenda: 23).

Agrarpolitisch motivierte Transferzahlungen beeinflussen die Erwartungshaltung von Produzenten gegenüber ihrem zukünftigen Einkommen. BURFISHER und HOPKINS (2003) unterscheiden zwischen Erwartungen hinsichtlich der zukünftigen Förderfähigkeit und der Höhe der Transferzahlungen. Erwartungen hinsichtlich der Förderfähigkeit von Betrieben 
haben einen direkten Einfluss auf die Produktionsentscheidungen und auf die Entscheidung zum Verbleib im Sektor. Betriebe, die die aktive Landwirtschaft einstellen, können nicht mehr in den direkten Genuss von Transferzahlungen gelangen. In der Vergangenheit wurden Transferzahlungen häufig in Abhängigkeit vom Produktionsumfang förderfähiger Kulturen bzw. Produkte festgelegt. Selbst unter einem entkoppelten Direktzahlungsregime werden weniger übliche Kulturen kaum angebaut, da hiermit ein Risiko des Verlustes der Förderfähigkeit verbunden ist (GOODWIN und MISHRA, 2002). Erwartungen hinsichtlich der Höhe von Direktzahlungen schlagen sich unter anderem in Bodenpreisen nieder. Für Betriebe mit eigenem Boden führt dies zu einer Erhöhung des Vermögens, was v. a. die Konsum- und Sparquote sowie den Umfang der außerbetrieblichen Erwerbsarbeit beeinflussen dürfte.

Auch unregelmäßig und ex-post gewährte Transferzahlungen, z. B. zum Ausgleich von Hochwasser- und Trockenschäden, haben indirekt einen Einfluss auf die Produktion. Jedem größeren Hochwasser- und Trockenheitsereignis folgten in der Vergangenheit staatliche Kompensationszahlungen zum Ausgleich der hierdurch entstandenen Schäden. Es gibt keinen Grund anzunehmen, dass dies in der Zukunft nicht genauso sein wird (YounG und Westscott, 2000). Ohne diese Zahlungen würden risikoaverse Produzenten besonders hochwasser- oder trockenheitsgefährdete Flächen nicht mehr oder anders nutzen oder sich gegen das Ausfallrisiko durch eine private Versicherung schützen.

Theoretisch sollten Agrarumweltmaßnahmen einen neutralen oder negativen Effekt auf die Produktion haben, da in erster Linie extensive Bewirtschaftungsformen gefördert werden. Für ausgewählte Agrarumweltmaßnahmen, wie dem Ökologischen Landbau, sind die überwiegend negativen Produktionseffekte bereits gut dokumentiert (NIEBERG, 1996; Nieberg und Offermann, 2006; SAlhofer und Streicher, 2005). Studien zur Produktionswirkung anderer Agrarumweltmaßnahmen (den Ökologischen Landbau ausgenommen) liegen bisher kaum vor. Theoretisch ist ein produktionsfördernder Effekt von Agrarumweltmaßnahmen möglich, wenn die kompensatorisch angelegten AUM-Zahlungen die tatsächlichen Opportunitätskosten der Teilnahme deutlich übersteigen (vgl. Kapitel 3.2.2). PufAhl und WeIss (2009) zeigen, dass die Teilnahme an Agrarumweltmaßnahmen in Deutschland einen positiven, wenn auch geringen Einfluss auf die Produktion hat.

\subsection{Zusammenfassung}

Kapitel 4 gibt einen Überblick über die empirischen Befunde zu den Wirkungen von direkten Transferzahlungen (ge- und entkoppelte Direktzahlungen, struktur- und umweltpolitische Programme) auf den betrieblichen Faktoreinsatz, die Produktion und die außerbetriebliche Erwerbsarbeit. 
Direkte Transferzahlungen haben in der Tendenz einen strukturkonservierenden Effekt: je höher die Transferzahlungen, desto niedriger ist Wahrscheinlichkeit der Aufgabe eines Betriebes bzw. desto geringer ist der Rückgang der Anzahl landwirtschaftlicher Betriebe. Dieser Zusammenhang gilt vor allem für ge- und entkoppelte Direktzahlungen, aber auch für umweltorientierte Programme. Die empirischen Befunde lassen insgesamt auf einen positiven Zusammenhang zwischen direkten Transferzahlungen und der Betriebsfläche, dem Anbauumfang geförderter Kulturen bzw. dem betrieblichen Größenwachstum schließen.

Die empirischen Ergebnisse zum Einfluss von (ge- und entkoppelten) Direktzahlungen auf die Allokation der Arbeitszeit zwischen betrieblicher und außerbetrieblicher Tätigkeit ergeben ein heterogenes Bild. Der Einfluss von Direktzahlungen auf den betrieblichen Arbeitseinsatz scheint in der Tendenz neutral bis positiv zu sein. Dagegen nimmt die Wahrscheinlichkeit bzw. der Umfang der außerbetrieblichen Arbeit mit steigenden Direktzahlungen zu. Inwieweit es sich hierbei um einen Gewinn- oder Substitutionseffekt zwischen außerbetrieblicher und betrieblicher Tätigkeit handelt, bleibt in der Regel unklar, da nur wenige Studien beide Aspekte (betriebliche und außerbetriebliche Arbeit) gleichzeitig untersuchen. Umwelt- und strukturpolitische Programme scheinen kaum einen Einfluss auf die betriebliche oder außerbetriebliche Erwerbstätigkeit des Betriebsleiterpaares zu haben. EL-OsTA et al. (2004: 383) schlussfolgern, dass Direktzahlungen ,...generally worked against the major farm and off-farm labor trends, though their impacts have been dwarfed by the main trends".

Die Veränderung des umweltrelevanten Faktoreinsatzes (Viehdichte, Pflanzenschutz- und Düngemittelaufwand je Hektar LF) ist primär für Programme mit umweltrelevanten Zielen, wie z. B. für Agrarumweltmaßnahmen und das Conservation Reserve Program, von Bedeutung. Die wenigen vorliegenden quantitativen Studien zeigen, dass die Nutzungsintensität infolge der Programmteilnahme abnimmt und der Anteil umweltfreundlicher Bodennutzungsformen zunimmt. Die im Mittel aller teilnehmenden Betriebe vorhandenen positiven Wirkungen von Agrarumweltmaßnahmen in Deutschland sind allerdings zu relativieren. Agrarumweltmaßnahmen werden häufig auf bereits extensiv bewirtschafteten Flächen angewendet. Die hierdurch induzierte Änderung der Nutzungsintensität ist in vielen Fällen gering bzw. geht gegen Null.

Einen positiven Effekt auf das Produktionsvolumen haben vor allem gekoppelte Direktzahlungen. Empirische Studien zeigen, dass auch entkoppelte Direktzahlungen und agrarumweltpolitisch motivierte Zahlungen in der Praxis durchaus mit positiven, wenn auch geringen Produktionseffekten verbunden sein können. Im Vergleich zu gekoppelten Direktzahlungen ist der Einfluss entkoppelter Direktzahlungen auf die Produktion eher indirekter Natur (Versicherungs- und Liquiditätseffekt). 


\section{Methoden für die Evaluation von politischen Programmen}

"An observational study differs from an experiment in that the random assignment of treatments (i. e. agents, programs, procedures) to units is absent. ... [T] his randomization is a powerful tool in that many systematic sources of bias are made random " (COCHRAN und RUBIN, 1973: 417).

Anhand des Zitates von COCHRAN und RUBIN (1973) lassen sich die Vorteile experimenteller Ansätze für die Evaluation von Programmen verdeutlichen: Die zufällige Auswahl von Individuen (Personen, Haushalten etc.) zur Programmteilnahme sichert die Gleichverteilung der Merkmale in der Gruppe der Teilnehmer und Nichtteilnehmer. Beide Gruppen unterscheiden sich im Mittel nur hinsichtlich ihres Teilnahmestatus, nicht jedoch hinsichtlich ihrer anderen (un-)beobachteten Merkmale. Der Effekt der Programmteilnahme kann im Experiment als Mittelwertdifferenz beider Gruppen gebildet werden (HECKMAN, 1992).

Experimente zur Evaluation direkter Transferzahlungen an landwirtschaftliche Betriebe wurden bisher nicht durchgeführt und sind aus politischen Gründen vermutlich auch zukünftig nicht durchführbar. Auf die Literatur zu experimentellen Verfahren wird in dieser Arbeit daher nicht eingegangen. Retrospektiv angelegte agrarökonomische Wirkungsanalysen besitzen daher immer den Charakter einer observational study oder nichtexperimentelle Studie und müssen sich mit der Kernproblematik von Evaluationen auseinandersetzen: Da die Programmteilnahme nicht das Ergebnis eines zufälligen Auswahlprozesses ist, unterscheiden sich Teilnehmer und Nichtteilnehmer bereits vor der Programmdurchführung systematisch hinsichtlich ihrer (un-)beobachteten Merkmale voneinander. Es liegt ein Selektionseffekt (selection bias) vor, der bei der Quantifizierung des Programmeffektes zu berücksichtigen ist.

Eine formale Beschreibung der Theorie kausaler Effekte und des Selektionseffektes ${ }^{17}$ erfolgt in Kapitel 5.1. Kapitel 5.2 stellt die in der Literatur diskutierten nichtexperimentellen Schätzer in Grundzügen vor. Daran gespiegelt werden die in der agrarökonomischen Evaluationspraxis eingesetzten Verfahren (Kapitel 5.3). Kapitel 5.4 fasst die Ergebnisse des methodisch orientierten Literaturüberblicks zusammen.

17

Weitere Ursachen für Selektionseffekte sind (neben der Selbstselektion zur Programmteilnahme): Die nicht-zufällige Auswahl einer Stichprobe aus der Grundgesamtheit sowie systematische Antwortausfälle für einzelne Variablen (item attrition) und Untersuchungszeitpunkte (panel attrition) (vgl. HSIAO, 2003). Methoden zur Kontrolle dieser Selektionseffekte sind nicht Gegenstand dieser Arbeit. Hierfür sei auf die Arbeiten von HECKMAN (1990), VERBEEK und NiJMAN (1992), WoOldRIDGE (1995), HSIAO (2003) sowie CAMERON und TRIVEDI (2005) verwiesen. 
Für die Evaluation von Programmen werden am häufigsten der mittlere Effekt der Programmteilnahme (ATE: Average Treatment Effect) und der mittlere Effekt der Programmteilnahme auf die Teilnehmer (ATT: Average Treatment Effect on the Treated) genutzt. Der ATE geht zurück auf RosENBAUM und RUBIN (1983) und beschreibt den über alle Teilnehmer und Nichtteilnehmer gemittelten Effekt der Programmteilnahme. Da die meisten Programme auf spezifische Teilnehmergruppen abzielen, ist der ATE nur bedingt zur Beurteilung von Programmen geeignet (WoOLDRIDGE, 2002: 604 f.). Standardmäßig wird daher der ATT verwendet, der als mittlerer Effekt der Programmteilnahme in der Gruppe der Teilnehmer definiert ist und auch in dieser Arbeit im Mittelpunkt steht. ${ }^{18}$

\subsection{Theorie kausaler Effekte}

Die grundlegenden theoretischen Arbeiten zur Quantifizierung kausaler Effekte in nichtexperimentellen Studien stammen von NEYMAN (1923) und FISHER (1951), deren Arbeiten von RUBIN (1974) und Holland (1986) wieder aufgegriffen wurden. Als theoretische Basis der Evaluation von Programmeffekten dient in dieser Arbeit die Theorie kausaler Effekte, auch bekannt unter dem Namen potential outcome framework (RUBIN, 1974).

Der Effekt der Programmteilnahme wird im Vergleich zu einer Referenzsituation ohne Teilnahme gemessen. Hierfür ist die Frage zu beantworten, welches potenzielle Ergebnis ein Teilnehmer ohne die Programmteilnahme erreicht hätte. Das Ergebnis der Programmteilnahme ist mit $Y^{1}$, das Ergebnis im Fall einer Nichtteilnahme mit $Y^{0}$ bezeichnet. Der Indikator der Programmteilnahme, $D$, nimmt für Teilnehmer den Wert $D=1$ und für Betriebe ohne Teilnahme den Wert $D=0$ an. Der mittlere Effekt der Programmteilnahme auf einen Teilnehmer (ATT) entspricht der Differenz zwischen dem Ergebnis eines Teilnehmers mit und ohne Teilnahme:

$$
A T T=E\left(Y^{1}-Y^{0} \mid D=1\right)=E\left(Y^{1} \mid D=1\right)-E\left(Y^{0} \mid D=1\right) .
$$

Das potenzielle Ergebnis eines Teilnehmers im Fall der Nichtteilnahme $\left(Y^{0} \mid D=1\right)$ kann nicht beobachtet werden. Diese fehlende Information ist durch einen geeigneten alternativen Parameter zu ersetzen. Ein möglicher Kandidat hierfür ist das beobachtete Ergebnis eines Nichtteilnehmers $\left(Y^{0} \mid D=0\right)$.

18 Alternative Parameter sind der lokale, mittlere Effekt der Programmteilnahme (LATE: Local Average Treatment Effect) nach IMBENS und ANGRIST (1994) und der marginale Effekt der Programmteilnahme (MTE: Marginal Treatment Effect) nach HECKMAN und NAVARRO-LOZANO (2004). 
Die Entscheidung zur Teilnahme an agrarpolitischen Programmen erfolgt nicht zufällig (non random treatment assignment). Da die Programmteilnahme freiwillig ist und teilweise an Zugangsvoraussetzungen gebunden ist, wird sich ein Betriebsleiter nur dann für die Programmteilnahme entscheiden, wenn die Kosten der Teilnahme niedriger sind als der in Aussicht stehende Nutzen. Die Kosten und der Nutzen der Teilnahme hängen wiederum von Merkmalen des Betriebes und des Haushaltes ab. Teilnehmer und Nichtteilnahme unterscheiden sich hinsichtlich dieser (un-)beobachteten Merkmale schon bevor das Programm durchgeführt wird. Aus diesem Grund ist nicht anzunehmen, dass das Ergebnis von Teilnehmern und Nichtteilnehmern in der Referenzsituation ohne das agrarpolitische Programm gleich wäre $\left(E\left(Y^{0} \mid D=1\right) \neq E\left(Y^{0} \mid D=0\right)\right)$. Die Differenz beider Terme entspricht dem Selektionseffekt, der für eine unverzerrte Schätzung des Programmeffektes zu kontrollieren ist.

\subsection{Nicht-experimentelle Schätzer für die Programmevaluation}

Im Folgenden wird ein Überblick über die am häufigsten verwendeten nichtexperimentellen Schätzer gegeben. Primäres Anliegen ist es, das Prinzip der Verfahren sowie ihre Vor- bzw. Nachteile (Annahmen, Datenbedarf etc.) darzustellen. Für eine formale und vollständige Beschreibung der Methoden, ihrer Varianten sowie der Annahmen sei auf Wooldridge (2002), Heckman und Navarro-Lozano (2004), Heckman et al. (1989), Heckman und Navarro (2007), Heckman und Urzúa (2009) und CAmeron und TRIVEDI (2005) verwiesen.

Die Pionierarbeit in der Entwicklung nicht-experimenteller Schätzer wurde in der Arbeitsmarkt- und Bildungsforschung geleistet. Eine zusammenfassende Darstellung und Systematisierung verfügbarer Schätzer ist z. B. bei HECKMAN und RoBB (1985) zu finden.

Nicht-experimentelle Schätzer können nach verschiedenen Kriterien systematisiert werden. Für einen einführenden Überblick ist eine Unterscheidung in parametrische und nichtbzw. semi-parametrische Verfahren ausreichend (vgl. Abbildung 5.1). In parametrischen Verfahren wird der Einfluss der Programmteilnahme auf die Ergebnisvariable (z. B. die Betriebsgröße) modelliert, wobei der Einfluss (un-)beobachteter Faktoren kontrolliert wird. Der Effekt der Programmteilnahme auf das interessierende Ergebnis wird von den übrigen Einflussfaktoren getrennt (REINOWSKI, 2006). Zu den am häufigsten verwendeten parametrischen Verfahren in der Programmevaluation gehören Regressionsverfahren mit Kontrollvariablen, Kontrollfunktion, Instrumentvariablen und Fixed-Effekt-Modelle. 
Abbildung 5.1: $\quad$ Nicht-experimentelle Schätzer in der Programmevaluation

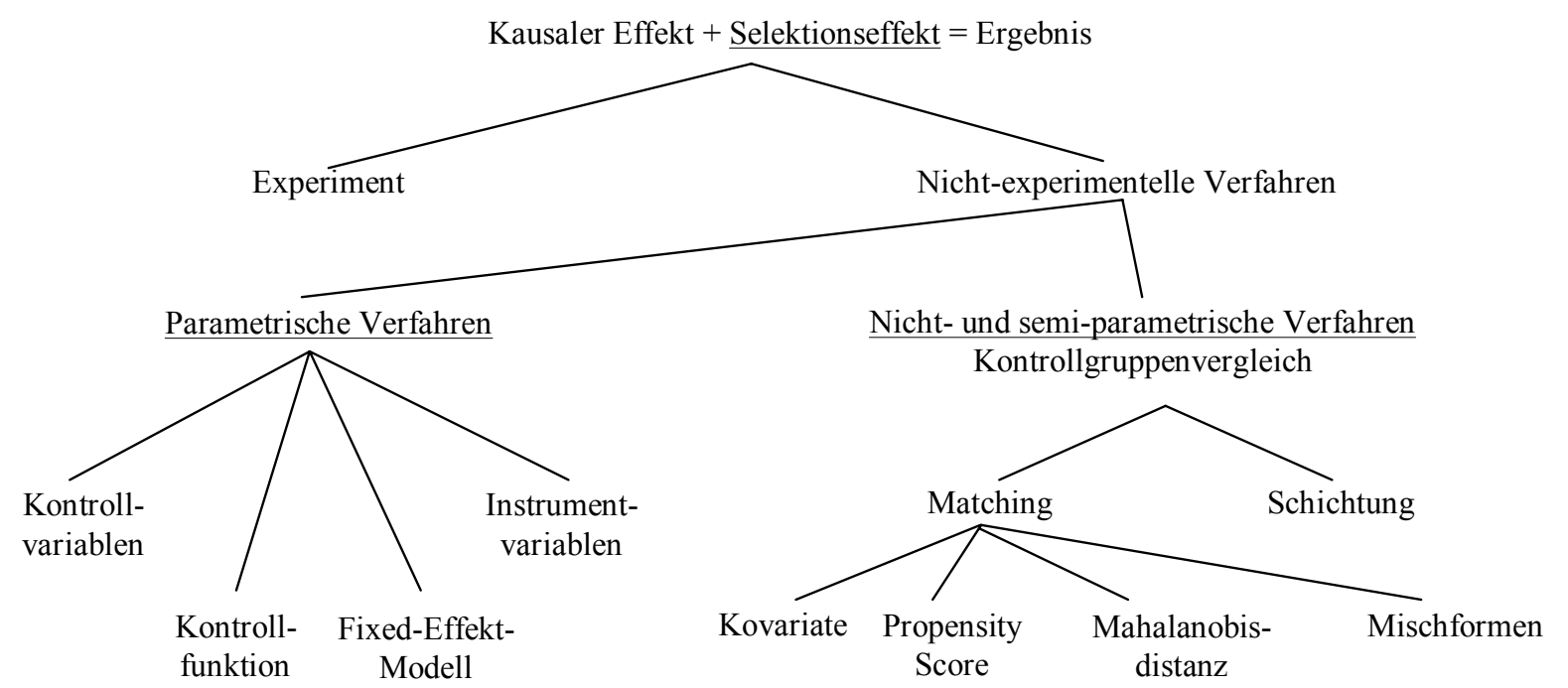

Quelle: nach CHRISTENSEN et al. (2005), geändert.

Nicht-parametrische Verfahren umfassen im Wesentlichen Kontrollgruppenvergleiche. ${ }^{19}$ Zur Konstruktion ähnlicher Kontrollgruppen können Schichtungs- und MatchingVerfahren eingesetzt werden. Im Matching werden einem (oder mehreren) Teilnehmer(n) ein (oder mehrere) ähnliche(r) Kontrollbetrieb(e) zugeordnet. Die Ähnlichkeit von Betrieben wird auf Basis einzelner Merkmale oder aggregierter Ähnlichkeitsmaße bestimmt. Ein besonders häufig verwendetes Ähnlichkeitsmaß ist der Propensity Score (RosENBAUM und RUBIN, 1983). Schichtungsverfahren sind vor allem dann geeignet, wenn die zu schichtenden Merkmale eine diskrete (nominale, ordinale) Skalierung aufweisen.

Die in Abbildung 5.1 dargestellten Verfahren können im Kontext von Querschnittsdaten (mit Beobachtungen für $i$ Betriebe zum Zeitpunkt $t$ ) und Paneldaten (mit Beobachtungen für $i$ Betriebe für $t$ Zeitpunkte mit $t \geq 2$ ) eingesetzt werden. Eine Ausnahme sind FixedEffekt-Modelle, für deren Schätzung Paneldaten notwendig sind.

Tabelle 5.1 stellt die Vor- und Nachteile von parametrischen und nicht-/semiparametrischen Verfahren dar. Zu den Vor- und Nachteilen parametrischer und nicht- bzw. semi-parametrischer Verfahren in der agrarökonomischen Politikevaluation hat sich jüngst eine Diskussion entwickelt (HenNing und MichaleK, 2008a, 2008b; MARgarian, 2008), auf die im abschließenden Kapitel 10 nochmals Bezug genommen wird.

19 Alternative nicht- und semi-parametrische Verfahren, z. B. nicht-parametrische Regressionsschätzungen, wurden in dieser Arbeit nicht explizit behandelt. Einen Überblick über neuere Entwicklungen in diesem Bereich geben ICHIMURA und TODD (2007). 
Tabelle 5.1: Vor- und Nachteile von parametrischen Verfahren und MatchingVerfahren

\begin{tabular}{lcc}
\hline Kriterien & Parametrische Verfahren & Matching-Verfahren \\
\hline Datenbedarf & mittel & hoch \\
Annahmen & hoch & gering \\
Flexibilität & hoch & mittel \\
Kommunizierbarkeit der Ergebnisse & schwer & einfach \\
\hline
\end{tabular}

Quelle: eigene Darstellung.

Datenbedarf: Nicht- und semi-parametrische Verfahren nutzen die zur Verfügung stehenden Informationen weniger effizient als parametrische Verfahren. Hieraus ergibt sich ein hoher Datenbedarf bei der Verwendung von nicht- und semi-parametrischen Verfahren.

Annahmen: Parametrische Verfahren beruhen auf restriktiven Annahmen hinsichtlich der Fehler- und Merkmalsverteilung und der korrekten funktionalen Spezifikation des empirischen Modells. In nicht- bzw. semi-parametrischen Verfahren bleibt der funktionale $\mathrm{Zu}-$ sammenhang zwischen den erklärenden Faktoren und der Ergebnisvariable weitgehend unspezifiziert; Verteilungsannahmen entfallen weitgehend. Weiterhin kann der Effekt der Programmteilnahme in nicht- bzw. semi-parametrischen Verfahren von Teilnehmer zu Teilnehmer variieren. In Anbetracht der Heterogenität landwirtschaftlicher Betriebe und unterschiedlicher Anpassungsstrategien an die Programmteilnahme, spiegelt ein individueller Programmeffekt die Realität am besten wider. In parametrischen Standardverfahren gilt die Annahme konstanter Programmeffekte, die jedoch durch eine geeignete Modellspezifikation aufgeweicht werden kann.

Flexibilität: In parametrischen Verfahren kann die interessierende Politikvariable jede beliebige Skalierung annehmen (diskret, stetig), während in Matching-Verfahren meist eine binäre Politikvariable (Teilnahme ja/nein) verwendet wird. Die Verwendung stetiger oder ordinal skalierter Politikvariablen in nicht- und semi-parametrischen Verfahren ist methodisch möglich, in der Regel aber aufwändig zu implementieren, da diese Option nicht in der verfügbaren Standardsoftware vorgesehen ist. Hypothesentests sind in parametrischen Verfahren (aufgrund der verfügbaren Software) häufig zeitsparender und flexibler durchzuführen als bei der Verwendung von Matching-Verfahren.

Kommunizierbarkeit der Ergebnisse: Ein Vorteil von nicht- und semi-parametrischen Verfahren ist die gute Kommunizierbarkeit der Ergebnisse an ein nicht-wissenschaftliches Publikum, da Mittelwertvergleiche einfacher zu verstehen sind als durch parametrische Verfahren geschätzte Koeffizienten. 
Darüber hinaus weisen die diskutierten Verfahren spezifische Vor- und Nachteile in Abhängigkeit von der Art der verfügbaren Daten auf. Zum Beispiel sind nicht- und semiparametrische Verfahren insbesondere dann geeignet, wenn die Gruppe der Teilnehmer im Vergleich zur Kontrollgruppe sehr klein ist. Parametrische Verfahren sind vorteilhafter, wenn die Anzahl der Teilnehmer- und Kontrollbetriebe in etwa gleich ist.

\subsubsection{Nicht- und semi-parametrische Schätzer}

Unter den nicht- und semi-parametrischen Verfahren besitzt das Matching die höchste praktische Bedeutung (CALIENDO, 2006; HeCKMAN et al., 1999; LECHNER, 2002). Die Identifikation des ATT beruht auf der Annahme, dass bei gegebenen, beobachteten Merkmalsausprägungen $X$, das potenzielle Ergebnis eines Betriebes unabhängig von seinem Teilnahmestatus ist: $Y^{0}, Y^{1} \perp D \mid X$. Diese Annahme ist unter dem Begriff Ignorability of Treatment Assumption (Rosenbaum und RUBIN, 1983) und Conditional Independence Assumption, CIA (LECHNER, 2002) bekannt. Hinter der CIA steht die Idee, dass das interessierende Ergebnis $(Y)$ als unabhängig vom Teilnahmestatus $(D)$ angesehen werden kann, wenn genügend Informationen in den beobachteten Merkmalen $(X)$ enthalten sind, um den Teilnahmestatus vorherzusagen (WoOLDRIDGE, 2002: 607).

Entsprechend der CIA ist nach der Berücksichtigung der beobachteten Unterschiede das mittlere potenzielle Ergebnis von Betrieben mit und ohne Teilnahme gleich $E\left(Y^{0} \mid D=1, X\right)=E\left(Y^{0} \mid D=0, X\right)$. Der mittlere Effekt des Programms auf die Teilnehmer entspricht dann

$$
A T T=E\left(Y^{1}-Y^{0} \mid D=1, X\right)=E\left(Y^{1} \mid D=1, X\right)-E\left(Y^{0} \mid D=0, X\right)
$$

Um das potenzielle Ergebnis eines Teilnehmers ohne Teilnahme zu approximieren, wird ein vergleichbarer Betrieb ohne Teilnahme mit gleichen beobachteten Merkmalen als Referenzgröße verwendet. Da $X$ ein Vektor beobachteter Merkmale ist, wird diese Methode auch als selection on observables ${ }^{20}$ bezeichnet (HECKMAN et al., 1989).

Die beschriebene Situation entspricht der eines Kreuzvergleiches: Teilnehmer und Kontrollbetriebe werden unter Berücksichtigung ihrer beobachteten Merkmale $X$ hinsichtlich ihres Ergebnisses $Y$ zum Zeitpunkt $t$ miteinander verglichen. Der Selektionseffekt wird nicht vollständig kontrolliert, wenn die Entscheidung zur Programmteilnahme auch auf

20

WOOLDRIDGE (2002: 608) verweist darauf, dass durch die Kontrolle beobachteter Unterschiede auch unbeobachtete Faktoren kontrolliert werden, sofern diese mit beobachteten Faktoren korrelieren. 
unbeobachtete Faktoren zurückzuführen ist und es Unterschiede im Ergebnisniveau zwischen Teilnehmern und Kontrollbetrieben gibt (SMITH und TODD, 2005).

Stehen Paneldaten zur Verfügung, so kann der Einfluss unbeobachteter Faktoren auf das Ergebnis teilweise durch einen Differenz-in-Differenz-Vergleich (DiD-Vergleich) kontrolliert werden (selection on observables). ${ }^{21}$ Im DiD-Vergleich wird nicht der absolute Wert des Ergebnisses $Y$ zwischen Teilnehmer- und Kontrollbetrieben verglichen, sondern die Ergebnisänderung $(\Delta Y)$ zwischen zwei Zeitpunkten. Durch die erste (betriebsspezifische) Differenzbildung wird der Einfluss unbeobachteter, zeitkonstanter betrieblicher Merkmale (z. B. Motivation und Managementfähigkeit des Betriebsleiters) auf das Ergebnis kontrolliert, da ein Betrieb quasi mit sich selbst verglichen wird. Durch die zweite Differenzbildung zwischen der Ergebnisänderung von Teilnehmer- und Kontrollbetrieben wird der Einfluss unbeobachteter, zeitvariabler Faktoren, die alle Betriebe gleichermaßen beeinflussen (z. B. Zinsentwicklung, gesamtwirtschaftliche Entwicklungen) kontrolliert. SMITH und TodD (2005) zeigen, dass der DiD-Vergleich in Verbindung mit Matching-Verfahren zu weniger verzerrten Schätzergebnissen führt als der Kreuzvergleich.

Unterscheiden sich Teilnehmer- und Kontrollbetriebe nur in wenigen (diskreten) Merkmalen, wie z. B. der Betriebsform und der Region, in der der Betrieb ansässig ist, so können Schichtungsverfahren zur Kontrolle des Selektionseffektes eingesetzt werden. Landwirtschaftliche Betriebe unterscheiden sich jedoch häufig in sehr vielen diskret und stetig skalierten Merkmalen. In diesem Fall ist es schwierig, genügend große Unterstichproben mit vergleichbaren Teilnehmer- und Kontrollbetrieben $\mathrm{zu}$ finden. ROSENBAUM and RUBIN (1983) zeigen, dass das Matching auf Basis des Propensity Scores $p(X)$ ausreichend ist, um eine Gleichverteilung der Merkmale zwischen beiden Gruppen zu erreichen. Der Propensity Score ist definiert als die aufgrund der beobachteten Merkmale $X$ bedingten Wahrscheinlichkeit eines Betriebes, als Teilnehmer klassifiziert $\mathrm{zu}$ werden: $p(X)=\operatorname{Pr}(D=1 \mid X)^{22}$

Eine weitere Voraussetzung für die Anwendung von Matching- und Schichtungsverfahren ist eine genügend große Anzahl von Teilnehmer- und Kontrollbeobachtungen mit gleichen beobachteten Merkmalen bzw. identischen Propensity Scores (Common Support Condition) (LECHNER, 2002). Sind die Merkmalsunterschiede zwischen Teilnehmer- und Kontrollbetrieben sehr hoch und/oder das Verhältnis zwischen der Anzahl der Teilnehmer- und

Der Einfluss unbeobachteter Effekte wird durch Differenzierung kontrolliert, sofern es sich um einen additiv separablen Effekt handelt. Additive Separabilität unterstellt, dass der Einfluss des unbeobachteten Effektes auf das interessierende Ergebnis nicht vom Niveau anderer Faktoren abhängt.

22 Schichtungs- und Matching-Verfahren können auch kombiniert werden, z. B. indem Betriebe zuerst nach betrieblichen Merkmalen geschichtet werden (Betriebsform, Größenklassen) und in jeder Schicht identische Teilnehmer- und Nichtteilnehmer einander zugeordnet werden. 
Kontrollbetriebe sehr eng, so kann nicht für jeden Teilnehmer ein Kontrollbetrieb gefunden werden. In der Konsequenz kann der Effekt der Programmteilnahme (ATT) nur für einen Teil der Teilnehmer geschätzt werden, was zu verzerrten Schätzergebnisse führen kann. Die Anzahl ähnlicher Teilnehmer- und Kontrollbetriebe sollte so groß sein, dass die Unterschiede zwischen beiden Gruppen durch statistische Tests abgesichert werden können.

\subsubsection{Parametrische Schätzer}

Alle vorgestellten parametrischen Verfahren erfordern die Spezifikation eines üblicherweise linearen Regressionsmodells. Die Ursache des Selektionseffektes kann in Regressionsmodellen als ein Problem einer unvollständigen Modellspezifikation interpretiert werden: Nicht alle Faktoren, die die Programmteilnahme und das interessierende Ergebnis beeinflussen, sind im Modell berücksichtigt, da diese unbeobachtet, unbekannt, nicht oder schwer messbar sind.

Ein lineares, univariates Regressionsmodell mit Programmeffekten ist beschrieben als

$$
y_{i}=d_{i} \delta+\varepsilon_{i} \quad i=1, \ldots, N,
$$

wobei $y_{i}$ das interessierende Ergebnis des iten Betriebes, $d_{i}$ der Indikator der Programmteilnahme und $\delta$ der interessierende Effekt der Programmteilnahme ist. Der zufällige Fehlerterm $\varepsilon$ hat eine unabhängige Verteilung mit konstanter Varianz und einem Erwartungswert von Null (identically and independently distributed, im Folgenden iid-verteilt). ${ }^{23}$ Der Indikator der Programmteilnahme kann stetig oder diskret skaliert sein. Da es nur einen Schätzer $\delta$ für den Effekt der Programmteilnahme gibt, ist der Effekt der Programmteilnahme für alle Betriebe gleich.

Der Effekt des Programms wird durch Gleichung (5.3) verzerrt gemessen, da es neben der Programmteilnahme weitere Einflussfaktoren gibt, die das interessierende Ergebnis oder die Programmteilnahme selbst beeinflussen. Diese in Gleichung (5.3) unberücksichtigten Faktoren verursachen eine Korrelation zwischen dem Fehlerterm und dem Indikator der Programmteilnahme; die Annahme iid-verteilter Fehler ist verletzt.

\footnotetext{
23 Zu weiteren Annahmen von linearen Regressionsmodellen vgl. V. AUER (2003) und WOOLDRIDGE
} (2002). 
Um einen unverzerrten Schätzer für die Programmteilnahme zu erhalten, müssen alle relevanten Faktoren mit Einfluss auf das interessierende Ergebnis und die Programmteilnahme kontrolliert werden, unabhängig davon, ob diese Faktoren beobachtet oder unbeobachtet sind. Hierfür werden üblicherweise Kontrollvariablen, Kontrollfunktionen, Instrumentvariablen und Fixed-Effekt-Modelle verwendet.

\section{Kontrollvariablen}

Das Regressionsmodell mit Kontrollvariablen ist das parametrische Pendant zum Kreuzvergleich beim Matching mit Kovariaten (selection on observables). Ein lineares, multivariates Regressionsmodell mit Programmeffekten ist beschrieben als

$$
y_{i}=x_{i} \beta+d_{i} \delta+\varepsilon_{i} \quad i=1, \ldots, N,
$$

wobei $y_{i}$ das interessierende Ergebnis, $x_{i}$ einen Vektor beobachteter Merkmale des Betriebes, $d_{i}$ die Programmteilnahme und $\varepsilon_{i}$ den iid-verteilten Fehlerterm repräsentieren. $\beta$ und $\delta$ sind Vektoren der zu schätzenden Parameter. Soweit nicht anders vermerkt, gilt für Gleichung (5.4) und alle folgenden Modelle die Annahme additiver Separabilität zwischen $y_{i}$ und $x_{i}$ bzw. $d_{i}$ und die Konstanz der Schätzer $\beta$ und $\delta$ über alle Beobachtungen.

Der Effekt der Programmteilnahme $\delta$ wird unverzerrt geschätzt, wenn $x_{i}$ alle relevanten Faktoren, die das interessierende Ergebnis und die Entscheidung zur Programmteilnahme beeinflussen, enthält. Sowohl das interessierende Ergebnis als auch die Programmteilnahme wird auch durch unbeobachtete (nicht messbare, unbekannte) Faktoren beeinflusst. Unbeobachtete Faktoren können wiederum eine Korrelation zwischen einem (mehreren) Regressor(en) und dem Fehlerterm verursachen.

\section{Kontrollfunktion}

Die Idee des Ansatzes der Kontrollfunktion ist es, die Korrelation zwischen dem endogenen Indikator der Programmteilnahme $d_{i}$ und dem Fehlerterm explizit zu modellieren und damit zu kontrollieren (HECKMAN und NAVARRo-LOZANO, 2004). Hierfür werden eine vollständige und eine reduzierte Ergebnisgleichung geschätzt:

$$
\begin{aligned}
& y_{i}=x_{i} \beta+d_{i} \delta+p \hat{v}_{i}+u_{i} \\
& d_{i}=z_{i} \xi+v_{i}
\end{aligned} \quad i=1, \ldots, N
$$

In der reduzierten Ergebnisgleichung wird die Programmteilnahme als Funktion der beobachteten Determinanten der Programmteilnahme $z_{i}$ geschätzt, wobei $z_{i}$ alle Elemente von $x_{i}$ enthalten kann (selection on observables) (HECKMAN et al., 1989). $\xi$ ist ein Vektor der zu schätzenden Parameter. Unbeobachtete Faktoren, die die Entscheidung zur Programmteilnahme beeinflussen, aber nicht in $z_{i}$ enthalten sind, schlagen sich im Fehler- 
term $v_{i}$ nieder. Der in der reduzierten Ergebnisgleichung geschätzte Fehler $\left(\hat{v}_{i}\right)$ wird als zusätzlicher Regressor in die Ergebnisgleichung eingesetzt und übernimmt die Rolle einer Kontrollfunktion. Unbeobachtete Faktoren der Programmteilnahme, die zuvor nicht kontrolliert wurden, werden durch $\hat{v}_{i}$ approximiert und damit kontrolliert.

Die vollständige Ergebnisgleichung (5.5) unterscheidet sich von Gleichung (5.4) nur hinsichtlich des zusätzlichen Regressors $\hat{v}_{i}$ und des hierfür zu schätzenden Parameters $p$. Hierdurch wird der Fehlerterm der Ergebnisgleichung in zwei Komponenten zerlegt $\left(\varepsilon_{i} \equiv p \hat{v}_{i}+u_{i}\right)$, wobei $p \hat{v}_{i}$ die Korrelation zwischen der Programmteilnahme und dem aggregierten Fehler $\varepsilon_{i}$ kontrolliert und $u_{i}$ den iid-verteilten Fehler der Ergebnisgleichung darstellt.

In Abhängigkeit von der Skalierung des Indikators der Programmteilnahme wird die reduzierte Ergebnisgleichung durch ein lineares Modell (stetige Skalierung), ein Tobit-Modell (stetig gestauchte Skalierung) oder durch ein Probit- oder Logit-Model1 ${ }^{24}$ (diskrete Skalierung) geschätzt.

\section{Instrumentvariablen}

Modelle mit Instrumentvariablen (IV-Modelle) können eingesetzt werden, wenn die Conditional Independence Assumption nicht gilt: In diesem Fall ist nicht anzunehmen, dass das Ergebnis von Teilnehmern und Nichtteilnehmern, nach Kontrolle der beobachteten Unterschiede, im Mittel gleich verteilt und damit unabhängig von der Programmteilnahme ist (WOOLDRIDGE, 2002: 621).

Vereinfachend wird angenommen, dass nur der Indikator der Programmteilnahme endogen, das heißt mit dem Fehlerterm korreliert ist. Ziel der Instrumentierung ist es, die Programmteilnahme vorauszuschätzen, nachdem der Einfluss weiterer beobachteter Kontrollvariablen eliminiert wurde. Hierfür ist mindestens ein Instrument $\left(z_{i}\right)$ notwendig, das i) mit der Programmteilnahme korreliert ist, ii) mit dem interessierenden Ergebnis unkorreliert ist und iii) nicht in den beobachteten Merkmalen $x_{i}$ enthalten ist (WoOLDRIDGE, 2002: 84).

Ein IV-Modell mit Kontrollvariablen und Programmeffekten ist beschrieben als

$$
\begin{aligned}
& y_{i}=x_{i} \beta+\hat{d}_{i} \delta+\varepsilon_{i} \\
& d_{i}=z_{i} \xi+v_{i}
\end{aligned} \quad i=1, \ldots, N
$$

${ }^{24}$ Ist die Programmteilnahme binär skaliert, so wird statt des Fehlers $\hat{v}_{i}$ das Inverse von Mills Ratio als Kontrollfunktion verwendet (HECKMAN, 1976). 
wobei $y_{i}$ das interessierende Ergebnis, $x_{i}$ die beobachteten Merkmale des Betriebes $i, \hat{d}_{i}$ die Vorausschätzung der Programmteilnahme und $\varepsilon_{i}$ den iid-verteilten Fehlerterm repräsentieren. Die beobachtete Programmteilnahme $\left(d_{i}\right)$ wird als Funktion der exogenen Instrumente $z_{i}$ geschätzt, wobei $z_{i}$ alle Elemente von $x_{i}$ und mindestens eine weitere exogene Variable enthalten muss. ${ }^{25}$ Auf Basis dieser Gleichung wird die Vorausschätzung der Programmteilnahme $\left(\hat{d}_{i}\right)$ ermittelt und anstelle von $d_{i}$ in die Ergebnisgleichung eingesetzt.

Für die Schätzung von IV-Modellen wird meist ein zweistufiges Verfahren mit einem Two Stage Least Squares (2SLS) Schätzer verwendet. 2SLS ist ein konsistenter Schätzer für den Effekt der Programmteilnahme und zwar unabhängig davon, ob $d_{i}$ binär, stetig oder gestaucht skaliert ist (ANGRIST, 1999: 3; WoOLDRIDGE, 2002: 92). Für den Fall einer binär skalierten, abhängigen Variablen kann alternativ das Dummy Endogenous Variable Modell (HECKMAN, 1978) verwendet werden.

\section{Fixed-Effekt-Modelle}

Eine Voraussetzung für die Anwendung von Fixed-Effekt-Modellen (FE-Modelle) ist die Verfügbarkeit von Paneldaten. FE-Modelle sind das parametrische Pendant zum Differenz-in-Differenz-Vergleich in Verbindung mit Matching-Ansätzen. Ein FE-Modell mit Kontrollvariablen und Programmeffekten ist beschrieben als (vgl. WoOLDRIDGE, 2002: 278)

$$
\begin{aligned}
& y_{i t}=x_{i t} \beta+d_{i t} \delta+\eta_{i}+u_{i t}, \quad i=1, \ldots, N, t=1, \ldots, T \\
& \varepsilon_{i t} \equiv \eta_{i}+u_{i t}
\end{aligned}
$$

wobei $y_{i t}$ das Ergebnis des $i$ ten Betriebes zum Zeitpunkt $t, x_{i t}$ ein Vektor der beobachteten Merkmale und $d_{i t}$ die Programmteilnahme repräsentieren. Die Vektoren der zu schätzenden Parameter sind durch $\beta$ und $\delta$ gekennzeichnet. Der Fehlerterm $\varepsilon_{i t}$ enthält den betriebsspezifischen (unbeobachteten) Effekt $\eta_{i}$ und den zufälligen, iid-verteilten Fehler $u_{i t}$. Der betriebsspezifische Effekt kann mit einem oder mehreren Elementen in $x_{i t}$ korrelieren (GREENE, 2003: 293); der Indikator der Programmteilnahme kann binär oder stetig skaliert sein. Elemente in $x_{i t}$ und $d_{i t}$ dürfen ausschließlich zeitvariable Faktoren, einschließlich jahresspezifischer Dummyvariablen, enthalten.

Das Prinzip der FE-Schätzung ist anhand der Gleichung (5.8) dargestellt:

$$
\left(y_{i t}-\bar{y}_{i .}\right)=\left(x_{i t}-\bar{x}_{i .}\right) \beta+\left(d_{i t}-\bar{d}_{i .}\right) \delta+\left(\eta_{i}-\bar{\eta}_{i .}\right)+\left(u_{i t}-\bar{u}_{i .}\right)
$$

25

Die Anzahl der zusätzlichen nicht in $x_{i}$ enthaltenen Instrumente $z_{i}$ muss mindestens der Anzahl der endogenen Variablen entsprechen. 
Die Schätzung erfolgt auf Basis der jährlichen Abweichungen $\left(y_{i t}, x_{i t}, d_{i t}, \eta_{i t}\right)$ vom über alle Untersuchungsjahre $t$ gebildeten, betriebsspezifischen Mittelwert $\left(\bar{y}_{i}, \bar{x}_{i}, \bar{d}_{i}, \bar{\eta}_{i}\right)$. Aus diesem Grund wird der FE-Schätzer auch als within-Schätzer ${ }^{26}$ bezeichnet. Durch die Differenzierung des betriebsspezifischen Effektes $\left(\eta_{i}-\bar{\eta}_{i}=0\right)$ wird der Einfluss zeitkonstanter, unbeobachteter Faktoren kontrolliert (selection on unobservables), sofern diese zeitkonstant und additiv separabel sind (WoOLDRIDGE, 2002: 638). Der Einfluss unbeobachteter, zeitvariabler Faktoren, die auf alle Betriebe gleichermaßen wirken, kann durch jahresspezifische Dummyvariablen kontrolliert werden.

Im FE-Modell wird der betriebesspezifische Effekt durch die within-Transformation der Daten kontrolliert. Ein alternativer Ansatz zur Kontrolle unbeobachteter, betriebspezifischer Effekte in Paneldaten ist das Einfügen von Dummyvariablen für jeden beobachteten Betrieb $i$. Dieses so genannte Least Square Dummy Variable Model (LSDV) führt zu identischen Schätzergebnisse wie das FE-Modell (WoOLDRIDGE, 2002: 272).

\subsection{Status quo der Wirkungsanalyse direkter Transferzahlungen}

In Tabelle 5.2 ist eine Auswahl von Methoden zusammengestellt, die für die (Ex-post-) Evaluation von direkten Transferzahlungen (ge- und entkoppelten Direktzahlungen, Umwelt- und Strukturprogrammen) verwendet werden. Tabelle 5.2 umfasst vorrangig Untersuchungen, die den Einfluss von direkten Transferzahlungen auf den Faktoreinsatz (einzelbetrieblich) bzw. die Faktorallokation (aggregierte Ebene) und die Produktion (Umsatz, Ertrag) untersuchen. Unter der Annahme, dass sich die ältere Literatur zu diesem Thema in der aktuellen Literatur widerspiegelt, sind vor allem Arbeiten der letzten zehn Jahre aufgeführt. Aufgrund der Vielzahl existierender Studien kann dieser Überblick nur unvollständig sein. von orthogonalen Abweichungen (orthogonal deviations) (ARELLANO und BOVER, 1995). 
Tabelle 5.2: $\quad$ Literaturüberblick - Zur Wirkungsanalyse direkter Transferzahlungen verwendete Methoden

\begin{tabular}{|c|c|c|c|c|c|}
\hline \multirow[t]{2}{*}{ Autor(en) } & \multicolumn{2}{|c|}{ Politik } & \multirow[t]{2}{*}{ Methode } & \multirow{2}{*}{$\begin{array}{l}\text { Kontrolle } \\
\text { Selektionseffekt }\end{array}$} & \multirow[t]{2}{*}{ Ergebnisvariablen } \\
\hline & $\mathbf{I}$ & II & & & \\
\hline \multicolumn{6}{|c|}{ Nicht- und semi-parametrische Verfahren } \\
\hline $\begin{array}{l}\text { BECK \& DOGOT (2006), BURGATH } \\
\text { et al. (2001), PLANKL et al. (2005) }\end{array}$ & & - & Kreuzvergleich & Schichtung & $\begin{array}{l}\text { Fläche (LF), Arbeit, } \\
\text { Einkommen }\end{array}$ \\
\hline $\begin{array}{l}\text { NIEBERG (1996), NIEBERG \& OFFER- } \\
\text { MANN }(2006), \text { OFFERMANN \& } \\
\text { NiEBERG }(2001 ; 2003)\end{array}$ & & - & Kreuzvergleich & $\begin{array}{l}\text { Kovariate } \\
\text { Matching }\end{array}$ & $\begin{array}{l}\text { Gewinn, Arbeit, Er- } \\
\text { trag, Produktion }\end{array}$ \\
\hline LYNCH et al. (2007a) & & $\mathbf{\square}$ & Kreuzvergleich & \begin{tabular}{|l|} 
Propensity Score \\
Matching \\
\end{tabular} & Pacht-/Bodenpreis \\
\hline SINABELL \& STREICHER (2005) & & - & DiD-Vergleich & Differenzierung & Ertrag \\
\hline $\begin{array}{l}\text { OSTERBURG \& STRATMANN (2002), } \\
\text { OSTERBURG (2005) }\end{array}$ & & - & DiD-Vergleich & $\begin{array}{l}\text { Differenzierung, } \\
\text { Euklidische } \\
\text { Distanz Matching }\end{array}$ & $\begin{array}{l}\text { Fläche (LF), Arbeit, } \\
\text { Viehbesatz, Einkom- } \\
\text { men }\end{array}$ \\
\hline PUFAHL \& WEISS $(2007 ; 2009)$ & & - & DiD-Vergleich & $\begin{array}{l}\text { Differenzierung, } \\
\text { Propensity Score } \\
\text { Matching } \\
\end{array}$ & $\begin{array}{l}\text { Fläche (LF), Arbeit, } \\
\text { Kapital, Viehbesatz, } \\
\text { Düngemitteleinsatz }\end{array}$ \\
\hline ROBERTS (2008) & घ & & DiD-Vergleich & $\begin{array}{l}\text { Differenzierung, } \\
\text { Propensity Score } \\
\text { Matching }\end{array}$ & $\begin{array}{l}\text { Fläche (einzelne } \\
\text { Kulturen) }\end{array}$ \\
\hline \multicolumn{6}{|l|}{ Parametrische Verfahren } \\
\hline MANN (2003), GIRANTE et al. (2008) & - & & OLS & Kontrollvariablen & \multirow{2}{*}{$\begin{array}{l}\text { Änderung der Fläche } \\
\text { (Betrieb, einzelne } \\
\text { Kulturen) \& Umsatz }\end{array}$} \\
\hline $\begin{array}{l}\text { KEY et al. (2005), LAMBERT \& GRIF- } \\
\text { FIN (2004), SALHOFER \& STREICHER } \\
\text { (2005), ADAMS et al. (2001), } \\
\text { GIRANTE et al. (2008) }\end{array}$ & घ & - & FE-Modell & $\begin{array}{l}\text { Differenzierung, } \\
\text { Kontrollvariablen }\end{array}$ & \\
\hline $\begin{array}{l}\text { PIETOLA et al. (2003), AHEARN et al. } \\
\text { (2006), CHANG \& BOISVERT (2005) }\end{array}$ & घ & & $\begin{array}{l}\text { Bivariates/ } \\
\text { Multinominales } \\
\text { Probit }\end{array}$ & Kontrollvariablen & $\begin{array}{l}\text { Außerbetriebliche } \\
\text { Arbeit, } \\
\text { Betriebsaufgabe } \\
\end{array}$ \\
\hline $\begin{array}{l}\text { AHEARN et al. (2002), } \\
\text { AHEARN et al. (2005) }\end{array}$ & घ & a & 3SLS, OLS & Kontrollvariablen & $\begin{array}{l}\text { Anzahl Betriebe, } \\
\text { Arbeit, Kapital etc. }\end{array}$ \\
\hline $\begin{array}{l}\text { GOODWIN et al. (2007), } \\
\text { EL-OSTA et al. (2004) }\end{array}$ & घ & - & Tobit \& OLS & IV-Modell & $\begin{array}{l}\text { Außerbetr. Arbeit, } \\
\text { Fläche (Betrieb, ein- } \\
\text { zelne Kulturen) }\end{array}$ \\
\hline $\begin{array}{l}\text { KEY \& ROBERTS (2006; 2003), } \\
\text { SERRA et al. (2005), HOFER (2002), } \\
\text { WEISS (2006b; 2006a) }\end{array}$ & घ & घ & $\begin{array}{l}\text { Logit-, Probit- } \\
\text { und Cox-Modell }\end{array}$ & Kontrollvariablen & $\begin{array}{l}\text { Außerbetr. Arbeit, } \\
\text { Betriebsaufgabe }\end{array}$ \\
\hline CHANG et al. (2008) & & - & Probit \& OLS & Kontrollfunktion & Einkommen \\
\hline LENCE \& MiSHRA (2003) & घ & & $\begin{array}{l}\text { Spatial Lag Mo- } \\
\text { dell }\end{array}$ & $\begin{array}{l}\text { Kontrollvariablen } \\
\text { Spatial Lags } \\
\end{array}$ & Pacht-/Bodenpreise \\
\hline BRÜMMER \& LOY (2000) & & & $\begin{array}{l}\text { Stochastic Fron- } \\
\text { tier Model }\end{array}$ & Kontrollvariablen & Effizienz \\
\hline ESPOSTI (2007) & घ & & GMM-Modell & $\begin{array}{l}\text { IV-Modell. Diffe- } \\
\text { renzierung, Kon- } \\
\text { trollvariablen }\end{array}$ & Änderung BWS \\
\hline MORO \& SCKOKAI (1999) & घ & & SEM & Kontrollvariablen & Produktion \\
\hline
\end{tabular}

Politik I: Gekoppelte und/oder entkoppelte Direktzahlungen, Politik II: Umwelt- und Strukturmaßnahmen (detailliert im Anhang, Tabelle A1), OLS: Ordinary Least Squares, 3SLS = Three Stage Least Squares, SEM: Simultanes Gleichungsmodell, FE-Modell: Fixed-Effekt-Modell, GMM-Modell: Modell mit General Methods of Moments-Schätzer, SEM: Simultanes Gleichungsmodell, DiD-Vergleich: Differenz-inDifferenz-Vergleich, BWS = Bruttowertschöpfung je Erwerbstätigen.

Quelle: eigene Zusammenstellung nach angegebenen Quellen. 
Aus Tabelle 5.2 ergibt sich folgendes Bild:

- Selektionseffekte aufgrund von Selbstselektion werden nicht immer explizit berücksichtigt oder, aufgrund der verwendeten Methoden, nur unzureichend kontrolliert. Der Einfluss der Politik auf ein interessierendes Ergebnis wird daher vermutlich häufig über- oder unterschätzt. Dies unterstreicht die bestehenden Potenziale bei der Weiterentwicklung der empirischen Methoden für agrarökonomische Wirkungsanalysen.

- Die Effekte von Direktzahlungen werden vorrangig durch parametrische Ansätze, die von Umwelt- und Strukturprogrammen eher durch nicht- und semi-parametrische Ansätze quantifiziert.

- Das Anliegen von Studien mit parametrischen Ansätzen ist häufig die Untersuchung einer Vielzahl möglicher Einflussfaktoren auf ein interessierendes Ergebnis unter denen Direktzahlungen ein Faktor sind. Der durch Selbstselektion verursachte Selektionseffekt wird in diesen Studien nur selten thematisiert oder explizit berücksichtigt.

- Die Selektivität von agrarstruktur- und agrarumweltpolitischen Instrumenten ist verhältnismäßig hoch, da die Programme auf bestimmte Ziele, Betriebe und Regionen ausgerichtet sind. Demzufolge ist die Notwendigkeit, den Selektionseffekt zu kontrollieren, hier am größten. Dies erfolgt meist explizit, durch die Verwendung nichtparametrischer (Schichtung, Kovariate Matching) oder semi-parametrischer Verfahren (Propensity Score Matching). Ein Schwachpunkt vieler nicht- und semiparametrischer Studien ist, dass die Schätzergebnisse nicht statistisch abgesichert werden.

- Die aufgeführten Untersuchungen basieren sowohl auf Querschnitts- als auch auf Paneldaten. Häufig erfolgt auch dann eine Querschnittsanalyse, wenn Paneldaten zur Verfügung stehen. Hierdurch wird die Möglichkeit vergeben, den Einfluss unbeobachteter Faktoren zumindest teilweise zu kontrollieren.

\subsubsection{Nicht- und semi-parametrische Ansätze}

Nicht- und semi-parametrischer Ansätze wurden bisher vor allem für die Evaluation von agrarumwelt- und strukturpolitischen Programmen (Agrarumweltmaßnahmen, Ausgleichszulage, Agrarinvestitionsförderung) verwendet (vgl. oberer Teil in Tabelle 5.2). Von RoBERTs (2008) liegt ein Anwendungsbeispiel des Matching-Ansatzes zur Evaluation von entkoppelten Direktzahlungen vor.

Am häufigsten angewendet wird der Kreuzvergleich, das heißt ein Vergleich zwischen Teilnehmern und Kontrollbetrieben $\mathrm{zu}$ einem festen Zeitpunkt (NIEBERG und OfFERMAnN, 2006; PlanKL et al., 2005). Der Kreuzvergleich ermöglicht die Kontrolle des Selektionseffektes durch beobachtete Faktoren (selection on observables). Unterschiede zwischen Teilnehmern- und Kontrollbetrieben aufgrund unbeobachteter Faktoren werden 
im Kreuzvergleich nur unzureichend kontrolliert. Eine methodische Verbesserung stellt die Verwendung eines Differenz-in-Differenz-Schätzers dar, da hierdurch teilweise der Einfluss unbeobachteter Faktoren auf das interessierende Ergebnis kontrolliert werden kann (selection on unobservables) (OSTERburg und Stratmann, 2002; Pufahl und WeISS, 2009; SinABELL und STREICHER, 2005).

Zur Evaluation der Agrarinvestitionsförderung werden darüber hinaus auch VorherNachher-Vergleiche eingesetzt (ORTNer, 2005; Pfefferli, 2006). Da ein und derselbe Betrieb an zwei verschiedenen Zeitpunkten verglichen wird, tritt im eigentlichen Sinn kein Selektionseffekt auf. Die kontrafaktische Situation, die ohne das Programm eingetreten wäre, bleibt jedoch unberücksichtigt, da der Einfluss exogener Faktoren (Preisentwicklungen, technologischer Fortschritt) nicht berücksichtigt wird.

Die zentrale methodische Frage des Kontrollgruppenvergleichs ist die Auswahl einer Kontrollgruppe, die der Teilnehmergruppe in vielen Merkmalen möglichst ähnlich ist. Die Bandbreite der hierfür verwendeten Methoden ist groß: PLANKL et al. (2005) nehmen für die Evaluation der Ausgleichszulage eine Schichtung von Teilnehmern und Nichtteilnehmern anhand mehrerer Kriterien ${ }^{27}$ vor. NIEBERG und OFFERMANN (2006) verwenden für die Auswahl konventioneller Vergleichsbetriebe sechs Merkmale ${ }^{28}$, die als unabhängig vom System „Ökologischer Landbau“ anzusehen sind. Jedem ökologisch wirtschaftenden Betrieb werden alle konventionellen Betriebe mit gleichen bzw. im definierten Rahmen abweichenden Merkmalsausprägungen zugeordnet. Trifft dies auf mehrere konventionelle Betriebe zu, so wird ein hypothetischer Kontrollbetrieb aus dem ungewichteten Durchschnitt aller verfügbaren konventionellen Vergleichsbetriebe gebildet. Die von NIEBERG und OfFERMAnN (2006) sowie Nieberg et al. (2007) verwendete Methode entspricht dem Matching auf Basis von Kovariaten (vgl. Abbildung 5.1).

Unterscheiden sich Teilnehmer und Nichtteilnehmer an einem Programm hinsichtlich vieler beobachteter Merkmale, so ist das Auffinden eines vergleichbaren Kontrollbetriebes ein mehrdimensionales Optimierungsproblem. Dieses Problem kann durch die Verwendung eines eindimensionalen Ähnlichkeits- oder Distanzmaßes vereinfacht werden. Osterburg und Stratmann (2002) setzen für die Auswahl von Kontrollbetrieben die Euklidische Distanz ein. In der Evaluationsliteratur wird hierfür standardmäßig der Propensity Score verwendet (vgl. HeCKMAN et al., 1999). Der Propensity Score ist definiert als die bedingte Wahrscheinlichkeit eines Betriebes, als Teilnehmer klassifiziert zu

27 Bundesland, Betriebsform, LF Betrieb, Landwirtschaftliche Vergleichszahl (LVZ), Art des benachteiligten Gebietes (Kleines Gebiet, Benachteiligte Agrarzone, Berggebiet).

28 
werden. Anwendungen des Propensity Score Matching in der agrarökonomischen Wirkungsanalyse sind z. B. in den Arbeiten von HeNNing und MiCHALEK (2008b), LyNCH et al. (2007a), Pufahl und Weiss (2009) und XiANPING und LyNCH (2006) zu finden.

Ein Schwachpunkt vieler empirischer Anwendungen von nicht- und semi-parametrischen Verfahren ist, dass weder die Vergleichbarkeit von Teilnehmern und Kontrollbetrieben (die Gleichverteilung der relevanten Merkmale), noch die geschätzten Programmeffekte statistisch abgesichert werden. Häufig ist die Stichprobe der Teilnehmer- und Kontrollbetriebe zu klein, um geeignete Testverfahren (T-Test, U-Test etc.) anzuwenden. In diesem Fall stellt sich auch die Frage nach der Belastbarkeit der geschätzten Ergebnisse.

\subsubsection{Parametrische Ansätze}

Am häufigsten für die Politikanalyse eingesetzt werden Fixed-Effekt-Modelle, mit deren Hilfe der Einfluss beobachteter und teilweise unbeobachteter (zeitkonstanter, betriebsindividueller) Faktoren auf das interessierende Ergebnis kontrolliert werden kann (selection on unobservables). Anwendungsbeispiele liegen vorrangig auf einzelbetrieblicher (GIRANTE et al., 2008; KEY et al., 2005), aber auch auf aggregierter Ebene (ADAMS et al., 2001) vor. Querschnittsmodelle werden nur noch selten als eigenständige Untersuchungsmethode verwendet (vgl. MANN, 2003), da hierdurch lediglich der Einfluss beobachteter Faktoren auf das interessierende Ergebnis kontrolliert werden kann (selection on observables). Häufig werden OLS-Modelle als Referenzmodelle für FEModelle und GMM-Modelle ${ }^{29}$ eingesetzt (vgl. BOND, 2002; GIRANTE et al., 2008). MORO und ScKOKAI (1999) bzw. SHAik und HELMERs (2006) verwenden simultane Gleichungsmodelle basierend auf Querschnittsdaten bzw. Längsschnittdaten.

Zur Schätzung diskreter Entscheidungen wie der außerbetrieblichen Erwerbsbeteiligung (SERRA et al., 2005; WeISS, 2006b) und der Betriebsaufgabe (HOFER, 2002; WEISS, 2006a) werden Probit- oder logistische Regressionsmodelle verwendet. Ist das interessierende Ergebnis zensiert oder gestaucht skaliert, so kommen Tobit-Modelle zum Einsatz (GooDWIN et al., 2007; MisHRA und GoODwIN, 1997). In Abhängigkeit von der jeweiligen Fragestellung werden diese Grundmodelle modifiziert und/oder mit anderen Modellen kombiniert (vgl. Tabelle 5.2). 
Die Möglichkeit des Vorhandenseins eines Selektionseffektes wird häufig nicht überprüft und nicht explizit kontrolliert; selbst dann nicht, wenn das originäre Anliegen der Studie die Schätzung des Politikeffektes ist (AHEARN et al., 2006, 2005; GoODWIN et al., 2007). Insbesondere in der letztgenannten US-Studie ist ein unberücksichtigter Selektionseffekt zu vermuten, da die Analyse auf einzelbetrieblicher Ebene ansetzt und, neben ge- und entkoppelten Direktzahlungen, auch die wesentlich selektiveren Umweltprogramme berücksichtigt.

SALHOFER und StREICHER (2005) illustrieren das Vorliegen eines Selektionseffektes durch einen Mittelwertvergleich zwischen den Teilnehmern und Nichtteilnehmern an Agrarumweltmaßnahmen. Der Selektionseffekt und der Einfluss weiterer Einflussgrößen auf das interessierende Ergebnis (Ertrag) wird durch die Schätzung eines klassischen FEModells kontrolliert. Eine explizite Kontrolle des Selektionseffektes erfolgt bei LAMBERT et al. (2006), die die Teilnahmeentscheidung von Betrieben am Conservation Reserve Program durch exogene Variablen instrumentieren.

LAMBERT und GRIFFIN (2004) nutzen ein FE-Modell mit räumlichen Effekten zur Erklärung des Pachtpreises. Die Politikvariablen werden als zeitliche Lags (frühere Werte der Politikvariablen) im Modell berücksichtigt, da der Effekt von Direktzahlungen vermutlich zeitverzögert auf den Pachtpreis wirkt. Der Einfluss unbeobachteter räumlicher Heterogenität auf den Pachtpreis wird durch die berücksichtigten räumlichen Effekte approximiert.

Eine über die Fragestellung dieser Arbeit hinausreichende Anwendung findet sich bei ESPOSTI (2007). Er verwendet ein dynamisches GMM-Model13 ${ }^{30}$, um den Effekt der Europäischen Strukturpolitik und der Gemeinsamen Agrarpolitik auf die Konvergenz europäischer Regionen zu untersuchen. GMM-Modelle verbinden die Vorteile von FE-Modellen und IV-Modellen: Zum einen werden endogene, erklärende Regressoren (Lags der abhängigen Variable, Politikvariable etc.) durch frühere Werte dieser Variablen instrumentiert. Zum anderen basiert die GMM-Schätzung vollständig bzw. teilweise auf differenzierten Daten, wodurch der Einfluss unbeobachteter Faktoren auf das interessierende Ergebnis kontrolliert wird. Darüber hinaus bilden dynamische Modelle wirtschaftliche Entwicklungen besser ab als statische Modelle, da Erstere die Historie der zu erklärenden Variable (z. B. das Ausgangsniveau der wirtschaftlichen Entwicklung) als erklärenden Faktor im Modell berücksichtigen. Dynamische Modelle werden bisher kaum zur Analyse agrarstruktureller Entwicklungen eingesetzt. 


\subsection{Zusammenfassung}

Der primär interessierende Parameter in Evaluationsstudien ist der mittlere Effekt der Programmteilnahme auf die Gruppe der Teilnehmer (ATT). Ist die Programmteilnahme nicht das Ergebnis eines zufälligen Auswahlprozesses, so unterscheiden sich Teilnehmer und Nichtteilnehmer bereits vor der Programmdurchführung systematisch voneinander (nonrandom treatment assignment). Es liegt ein Selektionseffekt aufgrund von Selbstselektion zur Programmteilnahme vor. Ohne die Berücksichtigung des Selektionseffektes wird der interessierende Politikeffekt verzerrt geschätzt. Theoretischen Überlegungen folgend ist der Selektionseffekt bei den agrarpolitischen Programmen am größten, die auf spezielle Ziele, Betriebe oder Regionen ausgerichtet sind. Dies betrifft vor allem agrarpolitische Struktur- und Umweltprogramme. Im Gegensatz dazu ist die Selektivität von ge- und entkoppelten Direktzahlungen deutlich geringer, da die meisten landwirtschaftlichen Betriebe - zumindest in der EU - Direktzahlungen erhalten.

Für viele empirische Fragestellungen können keine Experimente durchgeführt werden. Zur Quantifizierung von Programmeffekten werden daher parametrische und nicht- bzw. semiparametrische Verfahren eingesetzt, mit deren Hilfe der Selektionseffekt kontrolliert werden kann. In parametrischen Verfahren wird ein funktionaler Zusammenhang zwischen der Programmteilnahme und dem interessierenden Ergebnis geschätzt. Hierfür stehen verschiedene Verfahren (Kontrollvariablen, Kontrollfunktion, Instrumentvariablen, Differenzierung) zur Verfügung. Zu den nicht- bzw. semi-parametrischen Verfahren zählen alle Kontrollgruppenvergleiche: Ähnliche Teilnehmer und Nichtteilnehmer werden auf Basis von Schichtungs- und Matching-Verfahren ausgewählt und hinsichtlich ihrer Ergebnisse miteinander verglichen. Häufig stehen für empirische Wirkungsanalysen lediglich Querschnittsdaten zur Verfügung. Diese erlauben primär die Kontrolle des Selektionseffektes aufgrund beobachteter Faktoren (selection on observables). Das Prinzip der Differenzierung ermöglicht teilweise die Kontrolle unbeobachteter Faktoren (selection on unobservables), setzt allerdings die Verfügbarkeit von Paneldaten voraus.

Für die Evaluation agrarpolitischer Struktur- und Umweltprogramme kommen vorwiegend Kontrollgruppenvergleiche zum Einsatz, insbesondere der Kreuzvergleich in Verbindung mit Schichtungs- und Matching-Verfahren. Der zur Kontrolle unbeobachteter Faktoren notwendige Differenz-in-Differenz-Vergleich wird bisher nur selten genutzt. Ein Schwachpunkt vieler nicht- bzw. semi-parametrischer Anwendungen ist, dass die Ergebnisse nicht statistisch abgesichert werden. Für die Analyse des Einflusses von Direktzahlungen auf die Faktorallokation landwirtschaftlicher Betriebe werden primär parametrische Verfahren eingesetzt. Als Standardmethoden werden lineare Panelmodelle mit fixen Effekten (FE-Modelle) und Kontrollvariablen sowie diskrete, nicht-lineare Entscheidungsmodelle (Logit-, Probit- und Tobit-Modelle) mit Kontrollvariablen verwendet. 


\section{Datengrundlagen}

Für die empirische Analyse wird ein umfangreicher Datensatz einzelbetrieblicher Buchführungsdaten (Kapitel 6.1) verwendet, ergänzt um Daten der regionalen Agrar- und Wirtschaftsstatistik (Kapitel 6.2). Einen zusammenfassenden Überblick über die verwendeten Datengrundlagen gibt Kapitel 6.3.

\subsection{Buchführungsdaten}

Die Buchführungsdaten der Firma LAND-Data GmbH entsprechen inhaltlich denen des Testbetriebsnetzes des BMVEL (BMVEL, 2005). Der Datensatz enthält Informationen zu betrieblichen Merkmalen (Boden, Arbeit, Kapital, Viehbestand, Ausgaben für Dünge- und Pflanzenschutzmittel), zur Höhe der Programmzahlungen sowie zu den Charakteristika des Betriebsleiterpaares (Alter, Geschlecht, außerbetriebliche Arbeit). Tabelle 6.1 fasst die wichtigsten Merkmale der Ausgangsdaten (vollständige Stichprobe) und der für die empirische Analyse verwendeten Stichprobe zusammen.

Tabelle 6.1: $\quad$ Merkmale der verwendeten Stichprobe der Buchführungsdaten

\begin{tabular}{lrrr}
\hline Merkmal & Einheit & Vollständige Stichprobe & Verwendete Stichprobe \\
\hline Landwirtschaftliche Betriebe $(i)$ & $\mathrm{n}$ & 47.728 & 23.120 \\
Zeitpunkte $(T)$ & $\mathrm{n}$ & 1 bis 6 & 6 \\
Beobachtungen $(N)$ & $\mathrm{n}$ & 276.252 & 138.720 \\
Art des Panels & & unbalanciert & balanciert \\
Untersuchungszeitraum & & 2000 bis 2005 & 2000 bis 2005 \\
Zeitliche Erhebungseinheit & Wirtschaftsjahr & Wirtschaftsjahr \\
Räumlicher Bezug & Deutschland & Deutschland \\
Anteil an der Grundgesamtheit & & ca. $9 \%$ & ca. $5 \%$ \\
Repräsentativität & nein & nein \\
\hline
\end{tabular}

Quelle: eigene Darstellung.

Der Ausgangsdatensatz enthält ca. 276.000 Beobachtungen für 47.728 landwirtschaftliche Betriebe in Deutschland. Beobachtungen liegen für die Wirtschaftsjahre 1999/2000 bis 2004/2005 vor. Der Ausgangsdatensatz wurde um folgende Beobachtungen bzw. Betriebe bereinigt:

- Betriebe mit weniger als sechs Untersuchungsjahren (unvollständige Panels),

- Beobachtungen mit fehlenden Werten für einzelne Variablen, 
- Betriebe mit einer Betriebsgröße ab 2 ha LF, da nur Betriebe ab dieser Größe systematisch von der Agrarstatistik erfasst werden und die Agrarstatistik zur Beurteilung der Repräsentativität der Daten herangezogen wurde,

- Dauerkulturbetriebe, da diese für die Beurteilung von Agrarumweltmaßnahmen und der Ausgleichszulage eine geringe Relevanz haben,

- Ökologisch wirtschaftende Betriebe, da die Wirkungen des ökologischen Landbaus im Vergleich zu den anderen Agrarumweltmaßnahmen vermutlich sehr unterschiedlich $\operatorname{sind}^{31}$,

- Betriebe mit unplausiblen Werten (z. B. Milchproduktion ohne Milchkühe, negativer Faktoreinsatz bzw. Umsatz),

- Beobachtungen, für die aufgrund von Gebietsreformen und einer nicht aktualisierten Kreiskennung in den Buchführungsdaten eine Zuordnung zu einem Kreis nicht möglich war. Dies betrifft vorrangig Betriebe in Ostdeutschland und alle Betriebe des Bundeslandes Sachsen.

Für die empirische Analyse wird ein balanciertes Panel mit 23.120 Betrieben verwendet. Für jeden Betrieb liegen Beobachtungen für die Wirtschaftsjahre 1999/2000 bis 2004/2005 (2000 bis 2005), also für sechs Untersuchungsjahre, vor. Die Buchführungsdaten wurden entsprechend der Ausführungsanweisung des BMELV-Jahresabschlusses (BMELV 2006a) und der Deutschen Landwirtschaftsgesellschaft (DLG 2006) ausgewertet. Die verwendeten Variablen und deren Definition sind Tabelle A2 zu entnehmen.

Tabelle 6.2 gibt einen Überblick über die Repräsentativität der verwendeten Stichprobe, im Vergleich zur Grundgesamtheit aller landwirtschaftlichen Betriebe in Deutschland. Die Stichprobe umfasst etwa $5 \%$ aller landwirtschaftlichen Betriebe in Deutschland. Die Stichprobe ist nicht repräsentativ, da große Haupterwerbsbetriebe in Norddeutschland, insbesondere Veredlungsbetriebe, überrepräsentiert sind. Nebenerwerbsbetriebe, Betriebe unter 20 ha LF sowie Betriebe in Ostdeutschland sind in der Stichprobe kaum enthalten. Die verwendeten Buchführungsdaten repräsentieren etwa $4 \%$ aller landwirtschaftlichen Betriebe in benachteiligten Gebieten.

Die fehlende Repräsentativität ist vor allem auf den Selektionsmechanismus der Datenerhebung zurückzuführen: Die Buchführungsdaten der LAND-Data GmbH umfassen die Kunden der Buchführungsgesellschaft und stellen eine positive Selektion größerer und vergleichsweise gut aufgestellter Betriebe dar. Eine Übertragung der auf Basis dieser 
Stichprobe gewonnenen Ergebnisse auf die Grundgesamtheit der landwirtschaftlichen Betriebe in Deutschland ist nicht möglich.

Tabelle 6.2: Durch LAND-Data (2000) abgebildete Betriebe im Vergleich zur Grundgesamtheit (GG) landwirtschaftlicher Betriebe (1999)

\begin{tabular}{|c|c|c|c|c|}
\hline \multirow[t]{2}{*}{ Merkmal } & \multicolumn{2}{|c|}{ Betriebe ingesamt } & \multicolumn{2}{|c|}{ Betriebe in benachteiligten Gebieten } \\
\hline & $\begin{array}{c}\text { LAND-Data } \\
\text { Anzahl }\end{array}$ & $\begin{array}{c}\text { Anteil an GG } \\
\%\end{array}$ & $\begin{array}{c}\text { LAND-Data } \\
\text { Anzahl }\end{array}$ & $\begin{array}{c}\text { Anteil an GG } \\
\%\end{array}$ \\
\hline \multicolumn{5}{|l|}{ Region } \\
\hline Nord & 11.175 & 14,3 & 3.105 & 6,9 \\
\hline West & 1.623 & 1,7 & 929 & 2,2 \\
\hline Süd & 9.877 & 4,2 & 5.548 & 3,7 \\
\hline Ost & 445 & 1,8 & 218 & 1,7 \\
\hline \multicolumn{5}{|l|}{ Betriebsform } \\
\hline Marktfrucht & 4.835 & 3,3 & 1.182 & 1,9 \\
\hline Futterbau & 10.598 & 4,6 & 5.950 & 3,8 \\
\hline Veredlung & 3.076 & 10,7 & 819 & 6,1 \\
\hline Gemischtbetriebe & 1.698 & 5,7 & 528 & 2,8 \\
\hline \multicolumn{5}{|l|}{ Erwerbscharakter } \\
\hline Haupterwerbsbetriebe & 19.292 & 10,4 & 8.137 & 8,2 \\
\hline Nebenerwerbsbetriebe & 1.821 & 0,8 & 503 & 0,4 \\
\hline Kein Einzelunternehmen & 2.007 & 11,1 & 1.160 & 12,3 \\
\hline \multicolumn{5}{|l|}{ Betriebsgrößenklasse } \\
\hline unter 20 ha & 3.668 & 4,1 & 947 & 0,7 \\
\hline 20 bis 50 ha & 7.535 & 6,1 & 2.874 & 4,2 \\
\hline 50 bis 100 ha & 9.094 & 15,4 & 4.451 & 14,8 \\
\hline über 100 ha & 2.823 & 11,1 & 1.528 & 13,0 \\
\hline Gesamt & 23.120 & 5,4 & 9.800 & 3,9 \\
\hline
\end{tabular}

1. Landwirtschaftliche Betriebe ab 2 ha LF, ohne Dauerkulturbetriebe, ohne Öko-Betriebe.

2. Definition der Variablen vgl. Tabelle A2 und A3.

Quellen: eigene Auswertungen auf Basis der Mikrodaten der Agrarstatistik 1999 (STATISTISCHE ÄMTER, 2007) und LAND-DATA GMBH (2006).

Für die empirische Analyse werden ein Matching-Ansatz (differenziert für AUM und AZ) und verschiedene Panelmodelle verwendet. Die Definition des Teilnahmestatus und damit die Anzahl der analysierten Betriebe variiert in Abhängigkeit von der Analysemethode. Tabelle 6.3 zeigt die je Analysemethode verwendete Stichprobe und deren Unterschiede hinsichtlich der Definition des Teilnahmestatus von Betrieben. Die deskriptive Statistik der einzelnen Stichproben ist in den Tabellen A4 (Matching) und 9.1 (Panelregression) dargestellt. 
Tabelle 6.3: Verwendete Stichproben der Buchführungsdaten und deren Unterschiede hinsichtlich des Status der Programmteilnahme

\begin{tabular}{lcrrr}
\hline Merkmal & Einheit & Matching (AUM) & Matching (AZ) & Panelregression \\
\hline Betriebe $(i)$ & $\mathrm{n}$ & 19.337 & 23.120 & 22.551 \\
Zeitpunkte $(T)$ & $\mathrm{n}$ & 6 & 6 & 6 \\
Beobachtungen $(N)$ & $\mathrm{n}$ & 116.022 & 138.720 & 135.306 \\
Status der Programmteilnahme ist zeitlich ... & konstant & konstant & variabel \\
Teilnehmer (AUM=1, AZ=1) & $\mathrm{n}$ & 10.013 & 7.462 & variabel \\
Nichtteilnehmer (AUM=0, AZ=0) & $\mathrm{n}$ & 9.324 & 15.658 & variabel \\
\hline
\end{tabular}

Quelle: eigene Darstellung.

\section{Definition der Teilnahme an Agrarumweltmaßnahmen und an der Ausgleichszulage}

Der definierte Teilnahmestatus eines Betriebs wird in der Matching-Analyse (Kapitel 8) für den gesamten Untersuchungszeitraum (2000 bis 2005) konstant gehalten. Die Definition des Teilnahmestatus erfolgt auf Basis der Programmzahlungen. AUMTeilnehmer (AUM=1) haben im Referenzjahr 2000 keine und zwischen 2001 bis 2005 kontinuierlich positive Programmzahlungen erhalten. AZ-Teilnehmer $(A Z=1)$ haben in allen Jahren zwischen 2000 und 2005 positive Programmzahlungen (die Ausgleichszulage) erhalten. Nichtteilnehmer an Agrarumweltmaßnahmen $(A U M=0)$ bzw. an der Ausgleichszulage ( $A Z=0$ ) haben in keinem Jahr zwischen 2000 und 2005 positive Programmzahlungen erhalten. Der Teilnahmestatus vor 2000 bleibt bei der Auswahl von Teilnehmern und Nichtteilnehmern unberücksichtigt.

In der Panelregression (Kapitel 9) darf der Teilnahmestatus eines Betriebes willkürlich zwischen den Untersuchungsjahren variieren. Ein Betrieb ist ein Teilnehmer (AUM=1, $\mathrm{AZ}=1$ ), wenn dieser im Jahr $t$ positive Programmzahlungen bekommt; Nichtteilnehmer (AUM=0, AZ=0) erhalten im Jahr $t$ keine Programmzahlungen. Die geringe Beobachtungszahl in der Unterstichprobe „Panelregression“, im Vergleich zur Unterstichprobe „Matching (AZ)“, ist auf fehlende Werte von ausschließlich in der Panelregression verwendeten Variablen zurückzuführen.

Die gewählte Definition des Teilnahmestatus ( $1=$ Teilnahme, $0=$ Nichtteilnahme $)$ ist sehr restriktiv und berücksichtigt nicht, dass sich die Gruppe der Teilnehmer durch eine unterschiedliche Höhe der Programmzahlungen unterscheidet. Abbildung 6.1 zeigt die Verteilung der Programmzahlungen (AUM-Zahlungen, AZ-Zahlungen) in den teilnehmenden Betrieben für das Jahr 2005. AUM- bzw. AZ-Teilnehmer erhalten positive Programmzahlungen (größer Null). Für beide Programme ist eine vergleichbare, bei Null gestauchte, 
linksschiefe Verteilung der Programmzahlungen zu erkennen. Der Einfluss der Höhe der Programmzahlungen auf den betrieblichen Faktoreinsatz wird im Rahmen der Analyse heterogener Programmeffekte (Kapitel 8.4 und 9.3) untersucht.

Abbildung 6.1: $\quad$ Verteilung der AUM- und AZ-Zahlungen (2005) in Euro/Hektar LF*
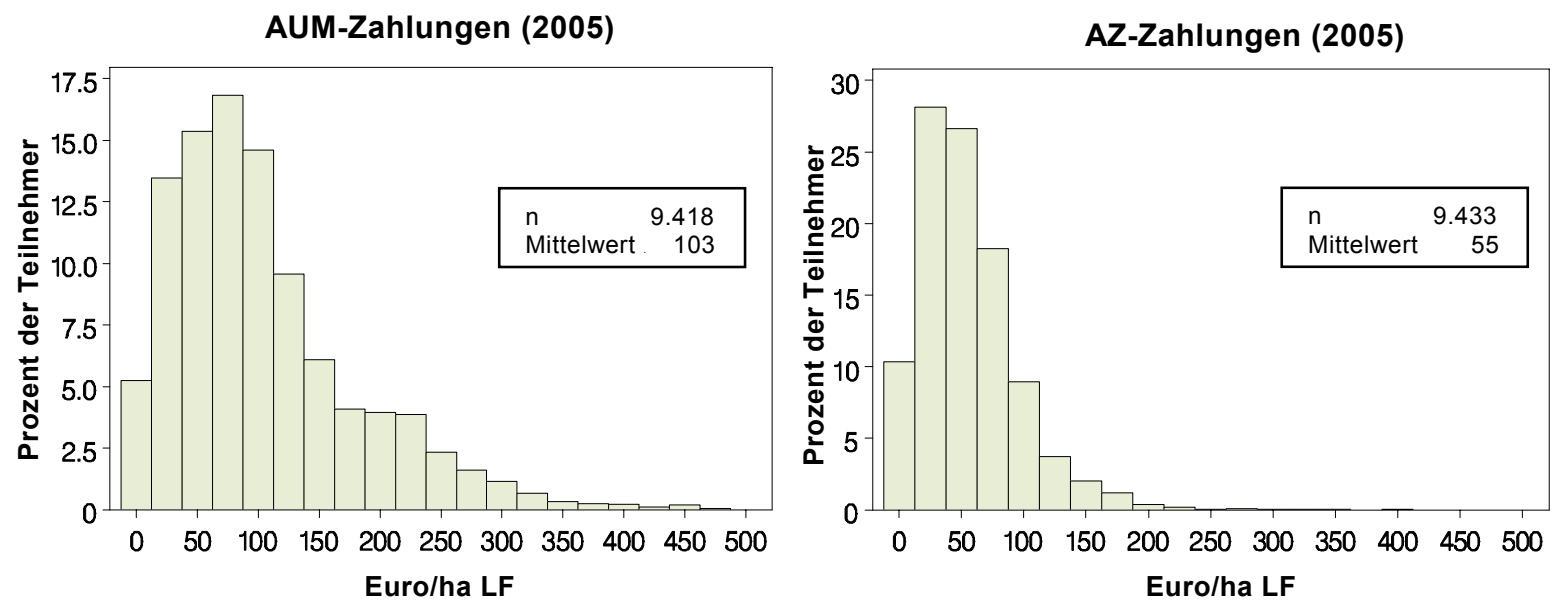

1. AUM-Zahlungen wurden bei 500 Euro/ha LF abgeschnitten.

2. * AUM- und AZ-Zahlungen/ha LF = Summe der vom Betrieb erhaltenen Zahlung/LF des Betriebs.

Quelle: eigene Berechnungen auf Basis von LAND-Data (2005).

\section{Grenzen der Buchführungsdaten hinsichtlich der Abbildung der Programmteilnahme}

Eine Unterscheidung verschiedener Agrarumweltmaßnahmen (Grünlandextensivierung, Vertragsnaturschutz etc.) ist auf Basis der Buchführungsdaten nicht möglich. ${ }^{32}$ Ebenso fehlen Informationen zum Umfang der tatsächlich geförderten Flächen, zur Prämienhöhe der geförderten Flächen sowie zur Art des benachteiligten Gebietes (Benachteiligte Agrarzone, Berggebiet, Kleines Gebiet). Aus diesem Grund ist die in Abbildung 6.1 dargestellte Höhe der Programmzahlung je Hektar LF eine berechnete Größe, die weder einen Rückschluss auf den Umfang der geförderten LF noch auf die gewährten Prämienzahlungen je Hektar erlaubt. Die berechneten Programmzahlungen je Hektar liegen deutlich unter den für AUM und AZ gewährten hektarbezogenen Prämiensätzen, da in der Regel nicht die gesamte LF des Betriebes durch AUM oder AZ gefördert wird.

Weiterhin kann auf Basis der Buchführungsdaten nur die Verringerung der (stofflichen) Faktorintensität nachvollzogen werden. Agrarumweltmaßnahmen honorieren jedoch auch die Verwendung mechanischer Verfahren (Mahd, Unkrautbekämpfung, Erosionsschutzver- 
fahren), die an bestimmte zeitliche Restriktionen gebunden sein können (z. B. keine Mahd vor dem 15. Juli). Die umweltrelevanten Effekte mechanischer Verfahren lassen sich durch Buchführungsdaten nicht abbilden.

\section{Verlässlichkeit und Konsistenz der Daten}

Aufgrund der großen Anzahl an Variablen ist grundsätzlich mit Mess-, Übertragungs- und Auswertungsfehlern zu rechnen. Die landwirtschaftliche Buchführung wird primär für steuerliche Zwecke erstellt, weshalb in einigen Punkten von einem strategischen Antwortverhalten auszugehen ist. Eine Einschätzung der Reliabilität der Buchführungsdaten gestaltet sich aufgrund der fehlenden Referenz schwierig. Standardmäßige Plausibilitätsprüfungen von Buchführungsdaten umfassen vor allem die Angaben zur Bilanz, die in dieser Arbeit jedoch eine sehr untergeordnete Bedeutung spielen. Im Rahmen eigener Plausibilitätsprüfungen wurden Betriebe mit unplausiblen Angaben (Milchproduktion ohne Milchkühe, negativer Faktoreinsatz bzw. Umsatz) von der Analyse ausgeschlossen.

Viele Betriebe wurden aufgrund fehlender Werte von der Analyse ausgeschlossen (vgl. Tabelle 6.1). Fehlende Werte traten insbesondere bei Variablen auf, die keine direkte Relevanz für die steuerliche Buchführung haben, aber Bestandteil des Erhebungsprogramms des Testbetriebsnetzes des BMVEL sind (z. B. (außer-)betrieblicher Arbeitsaufwand, Erzeugerpreise etc.). Dies könnte ein Hinweis darauf sein, dass auch die vorhandenen Angaben zu diesen Variablen die aktuelle Situation in den Betrieben nicht immer genau widerspiegeln.

\subsection{Sekundärdaten}

Zur Abbildung des sozioökonomischen, raum- und agrarstrukturellen Umfeldes landwirtschaftlicher Betriebe werden Daten der Agrar- und Regionalstatistik auf Ebene der Kreise und kreisfreien Städte verwendet. Tabelle 6.4 zeigt die wesentlichen Merkmale der verwendeten Sekundärdaten.

Eine Übersicht über die verwendeten Variablen und deren Definition ist in Tabelle A3 im Anhang zu finden. Die sozioökonomischen Daten umfassen z. B. die Bruttowertschöpfung, die Arbeitslosenquote, den durchschnittlich in der Industrie gezahlten Stundenlohn sowie die Pacht- und Bodenpreise einer Region. Daten zur Raumstruktur umfassen Angaben zur Besiedlungsdichte, zur Erreichbarkeit und Zentralität von Orten (BBR, 2003). Die agrarstrukturelle Situation wird durch die Daten der Agrarstatistik abgebildet.

Die Sekundärdaten werden über die fünfstellige Kreiskennung mit den Buchführungsdaten verknüpft. Aufgrund zahlreicher Gebietsreformen war dies in Ostdeutschland nur teilweise möglich und führte zum Verlust zahlreicher Beobachtungen. 
Tabelle 6.4: $\quad$ Merkmale der verwendeten Stichprobe der Sekundärdaten

\begin{tabular}{lcr}
\hline Merkmal & Einheit & Kontextdaten \\
\hline Kreise $(i)$ & $\mathrm{n}$ & 272 \\
Zeitpunkte $(T)$ & $\mathrm{n}$ & 6 \\
Beobachtungen $(N)$ & $\mathrm{n}$ & 1.632 \\
Art des Panels & & balanciert \\
Zeitlicher Bezug & & 2000 bis 2005 \\
Räumlicher Bezug & & Deutschland \\
Räumliche Erhebungseinheit & & Nuts III (Kreis) \\
Zeitliche Erhebungseinheit & & Kalenderjahr \\
Anteil an der Grundgesamtheit & & 80 \\
\hline
\end{tabular}

Quelle: eigene Darstellung.

\subsection{Zusammenfassung}

Für die empirische Analyse werden Buchführungsdaten (LAND-Data) von etwa 23.000 Betrieben verwendet, für die Beobachtungen für sechs Untersuchungsjahre (2000 bis 2005) vorliegen. Betriebe mit weniger als sechs Untersuchungsjahren wurden von der Analyse ausgeschlossen (balanciertes Panel). Die Stichprobe umfasst $5 \%$ aller landwirtschaftlichen Betriebe in Deutschland, ist jedoch nicht repräsentativ, da große Haupterwerbsbetriebe und Veredlungsbetriebe überrepräsentiert sind. Die Buchführungsdaten wurden um kreisbezogene Sekundärdaten (Wirtschafts- und Agrarstatistik) ergänzt.

Der Teilnahmestatus eines Betriebes an Agrarumweltmaßnahmen bzw. an der Ausgleichszulage (AZ) wird auf Basis der vom Betrieb erhaltenen Programmzahlungen bestimmt: Teilnehmer haben positive Programmzahlungen größer Null Euro erhalten und Nichtteilnehmer keine Programmzahlungen. In der Matching-Analyse werden, methodisch bedingt, nur Betriebe berücksichtigt, die - mit Ausnahme des Referenzjahres 2000 - immer oder nie an der Ausgleichszulage bzw. an Agrarumweltmaßnahmen teilgenommen haben. In der Panelregression darf der Teilnahmestatus eines Betriebes willkürlich zwischen den Untersuchungsjahren variieren. Aufgrund der unterschiedlichen Abgrenzung von Teilnehmern und Nichtteilnehmern variiert die Größe der für die jeweiligen Analysen verwendeten Stichproben geringfügig.

In den Buchführungsdaten fehlen Informationen zur Art der Agrarumweltmaßnahmen (Grünlandextensivierung etc.), zum Umfang der geförderten Fläche, zur Prämienhöhe je Hektar sowie zur Art des benachteiligten Gebietes. Die in dieser Arbeit verwendete Größe der Programmzahlungen je Hektar LF ist daher eine berechnete Größe, die keinen Rückschluss auf den Umfang der geförderten LF oder auf die Prämienhöhe je Hektar erlaubt. 


\section{Verwendete Methoden}

Zur Quantifizierung der Effekte der Teilnahme an Agrarumweltmaßnahmen und der Ausgleichszulage werden ein semi-parametrisches Matching-Verfahren (Kapitel 7.1) und parametrische Panelregressionsmodelle (Kapitel 7.2) verwendet. Beide methodischen Ansätze sind für Paneldaten mit vielen Beobachtungseinheiten und wenigen Beobachtungszeitpunkten geeignet. Der wesentliche Vorteil von Paneldaten gegenüber Querschnittsdaten liegt in der Möglichkeit, den Einfluss unbeobachteter Faktoren auf das interessierende Ergebnis zu kontrollieren.

Bereits in Kapitel 4.2 wurde darauf verwiesen, dass nicht- bzw. semi-parametrische Methoden auf weniger restriktiven Annahmen beruhen als parametrische Verfahren. Nachteile nicht- bzw. semi-parametrischer Methoden sind die weniger effiziente Ausnutzung der verfügbaren Informationen und die eingeschränkten Möglichkeiten für Hypothesentests. Welche Implikationen diese Vor- und Nachteile für die Anwendung der Methoden haben, wird in diesem Kapitel demonstriert. Zu diesem Zweck werden das Matching-Verfahren und die Panelmodelle auf die gleiche Fragestellung und auf den gleichen Datensatz angewendet.

\subsection{Propensity Score Matching}

Als Beispiel für ein semi-parametrisches Verfahren wird ein Kontrollgruppenvergleich in Verbindung mit dem Propensity Score Matching verwendet. Das Propensity Score Matching gehört in vielen wirtschafts- und sozialwissenschaftlichen Disziplinen zum methodischen Standardrepertoire (CALIENDO und KoPEINIG, 2008; HECKMAN et al., 1998; ROSENBAum und Rubin, 1983). Anwendungen des Propensity Score Matching für die agrarpolitischen Wirkungsanalysen in entwickelten Volkswirtschaften liegen von HENNING und MichaleK (2008b), Lynch und Xianping (2007), Pufahl und Weiss (2009) und XIANPING und LYNCH (2006) vor.

\subsubsection{Verwendetes Modell}

Der Ausgangspunkt des Matching-Modells ist die in Kapitel 4.1 dargestellte Theorie kausaler Effekte. Unterschieden werden Individuen (hier: landwirtschaftliche Betriebe) mit und ohne Programmteilnahme. Der Effekt der Programmteilnahme auf die Teilnehmer entspricht der Differenz zwischen dem beobachteten Ergebnis mit Programmteilnahme und dem hypothetischen Ergebnis, welches der Betrieb im Fall der Nichtteilnahme erreicht hätte. 
Die Programmteilnahme ist mit $D=1$, die Nichtteilnahme mit $D=0$ gekennzeichnet. $Y^{1}$ kennzeichnet das Ergebnis mit Programmteilnahme, $Y^{0}$ das Ergebnis im Fall der Nichtteilnahme am Programm. In Evaluationen interessiert zumeist der mittlere Effekt der Programmteilnahme auf die Teilnehmer, der Average Treatment Effect on the Treated (ATT),

$$
A T T=E\left(Y^{1}-Y^{0} \mid D=1\right)=E\left(Y^{1} \mid D=1\right)-E\left(Y^{0} \mid D=1\right) .
$$

Der ATT misst den durchschnittlichen Effekt des Programms in der Gruppe der teilnehmenden Betriebe. Der letzte Term der Gleichung - das hypothetische Ergebnis eines Teilnehmers im Fall der Nichtteilnahme - kann nicht beobachtet werden. Würde die Programmteilnahme zufällig erfolgen, so könnte $E\left(Y^{0} \mid D=1\right)$ durch das beobachtete Ergebnis der Nichtteilnehmer $E\left(Y^{0} \mid D=0\right)$ ersetzt werden: Der Erwartungswert des Ergebnisses von Teilnehmern und Nichtteilnehmern in der Referenzsituation ohne das Programm wäre gleich groß $\left(E\left(Y^{0} \mid D=1\right)=E\left(Y^{0} \mid D=0\right)\right)$. In diesem Fall würde die zufällig erfolgte Programmteilnahme - im Mittel - eine Gleichverteilung der betrieblichen Merkmale und des erwarteten Ergebnisses von Teilnehmern und Nichtteilnehmern sicherstellen.

Die Annahme einer zufälligen Programmteilnahme ist in Bezug auf die Evaluation agrarpolitischer Programme nicht haltbar. Da die Teilnahme freiwillig erfolgt und an Zugangsvoraussetzungen gebunden ist, wird sich ein Betriebsleiter nur dann für die Programmteilnahme entscheiden, wenn die Kosten der Teilnahme niedriger sind als der in Aussicht stehende Nutzen. Kosten und Nutzen der Programmteilnahme hängen wiederum von den Merkmalen des Betriebes und des Haushaltes ab, woraus systematische Unterschiede zwischen den Teilnehmern und den Nichtteilnehmern resultieren. Das Ergebnis von Teilnehmern und Nichtteilnehmern wäre in der Referenzsituation ohne das agrarpolitische Programm nicht gleich $\left(E\left(Y^{0} \mid D=1\right) \neq E\left(Y^{0} \mid D=0\right)\right)$. Die Differenz beider Terme entspricht dem Selektionseffekt, der für eine unverzerrte Schätzung des Programmeffektes zu kontrollieren ist.

Die von RUBIN (1974) vorgeschlagene Lösung basiert auf der Idee, die unterschiedlichen Merkmale von Teilnehmern und Nichtteilnehmern in der empirischen Analyse explizit zu berücksichtigen. RUBIN unterstellt, dass bei gegebenen betrieblichen Merkmalsausprägungen $(X)$ das hypothetische Ergebnis eines Betriebes unabhängig von seinem Teilnahmestatus ist, $Y^{0}, Y^{1} \perp D \mid X$ (Conditional Independence Assumption, CIA). Unter der CIA gilt, dass - nach Berücksichtigung der beobachteten Unterschiede - das mittlere potenzielle Ergebnis ohne Teilnahme von teilnehmenden und nicht teilnehmenden Betrieben gleich ist. In diesem Fall kann der letzte Term in Gleichung (7.1) durch das beobachtete Ergebnis eines Nichtteilnehmers mit gleichen Merkmalen ersetzt werden:

$$
A T T=E\left(Y^{1} \mid D=1, X\right)-E\left(Y^{0} \mid D=0, X\right)
$$


Um das hypothetische Ergebnis eines Teilnehmers ohne Teilnahme $\mathrm{zu}$ approximieren, wird ein vergleichbarer Betrieb ohne Teilnahme als Referenzgröße (Kontrollbetrieb) verwendet.

Rosenbaum und Rubin (1983) zeigen, dass das Matching auf Basis des Propensity Scores $p(X)$ ausreichend ist, um eine Gleichverteilung der Merkmale zwischen Teilnehmern und Kontrollbetrieben zu erreichen. Der Propensity Score ist definiert als die bedingte Wahrscheinlichkeit eines Betriebes, aufgrund der beobachteten Merkmalsausprägungen $X$ als Teilnehmer klassifiziert zu werden $p(X) \equiv \operatorname{Pr}(D=1 \mid X)$. Vergleichbare Teilnehmer und Nichtteilnehmer werden auf Basis eines identischen Propensity Score ausgewählt (selection on observables). Der ATT wird entsprechend Gleichung (7.3) geschätzt:

$$
A T T=E\left[Y^{1} \mid D=1, p(X)\right]-E\left[Y^{0} \mid D=0, p(X)\right]
$$

Der Propensity Score wird durch ein binäres Entscheidungsmodell, z. B. ein Logistisches Regressionsmodel (Logit-Modell), geschätzt. Im Logit-Modell wird die Entscheidung zur Programmteilnahme, z. B. an Agrarumweltmaßnahmen, modelliert. HECKMAN und RoBB (1985) verweisen auf die Bedeutung einer theoriebasierten Spezifikation des Entscheidungsmodells, um den Selektionseffekt möglichst umfassend zu kontrollieren.

Für den Vergleich mehrerer Programme empfiehlt LECHNER (2002) entweder die Schätzung des Propensity Score in mehreren separaten binären Logit-Modellen oder die Spezifikation eines multinominalen Logit-Modells. ${ }^{33}$ Da Letzteres das Risiko der Fehlspezifikation erhöht (ebd.), wird der Propensity Score für die Teilnahme an Agrarumweltprogrammen bzw. an der Ausgleichszulage in zwei separaten Logit-Modellen geschätzt.

Um für jeden Teilnehmer ein hypothetisches Nichtteilnahme-Ergebnis schätzen zu können, müssen genügend Kontrollbetriebe mit gleichen Merkmalsausprägungen vorhanden sein (Common Support Condition, CS). ${ }^{34}$ Neben der CIA und CS basiert das MatchingVerfahren auf den Annahmen, dass alle Teilnehmer bzw. Nichtteilnehmer ein identisches ${ }^{35}$ Treatment bzw. Non-Treatment erhalten und dass die Programmteilnahme nur die Teilnehmer beeinflusst und keine indirekten Wirkungen auf die Nichtteilnehmer hat (Stable

33 Weitere Matching-Ansätze zur Evaluation von multi-valued treatments vgl. IMBENS (2000).

34 Bezogen auf die empirische Analyse bedeutet dies, dass Teilnehmer und Nichtteilnehmer mit einer gleichen Faktorausstattung und vergleichbaren betriebswirtschaftlichen Kennzahlen im Datensatz vorhanden sein müssen, um für jeden Teilnehmer einen nicht teilnehmenden „Zwilling“ finden zu können.

35 Ein identisches Treatment bzw. Non-Treatment bezieht sich auf die Art des Programms oder die erhaltene Dosis eines Programms. Bezogen auf die empirische Fragestellung erhalten alle Betriebe, die mit $100 \%$ der LF an der Agrarumweltmaßnahme Grünlandextensivierung teilnehmen ein identisches Treatment. 
Unit Treatment Value Assumption, SUTVA). Das Zutreffen aller Annahmen wird vor dem Hintergrund der empirischen Modellspezifikation in Kapitel 8.1 diskutiert.

\section{Differenz-in-Differenz-Schätzung}

Der Selektionseffekt wird durch die Kontrolle der beobachteten Unterschiede zwischen den Merkmalen von Teilnehmern und Nichtteilnehmern in der Regel nicht vollständig beseitigt. SMITH und TODD (2005) verweisen auf eine Reihe möglicher Gründe, wie z. B. den Einfluss unbeobachteter Unterschiede oder Unterschiede des Ergebnisniveaus zwischen Teilnehmern und Kontrollbetrieben aus verschiedenen Regionen. Um die Ergebnisse des Matching zu verbessern, empfehlen SMITH und TODD (2005) die Verwendung eines Differenz-in-Differenz-Schätzers (DiD-Schätzer). Eine Voraussetzung für die Anwendung des DiD-Schätzers ist das Vorliegen von Paneldaten mit mindestens zwei Untersuchungszeitpunkten.

Hierfür wird Gleichung (7.3) um einen DiD-Schätzer erweitert. Das Subskript $t$ repräsentiert einen Zeitpunkt nach und $t^{\prime}$ einen Zeitpunkt vor dem Programmstart. Gemessen wird die Ergebnisänderung von vergleichbaren Teilnehmern und Kontrollbetrieben vor und nach der Programmdurchführung:

$$
A T T=E\left[Y_{t}^{1}-Y_{t^{\prime}}^{0} \mid D=1, p\left(X_{t^{\prime}}\right)\right]-\left[Y_{t}^{0}-Y_{t^{\prime}}^{0} \mid D=0, p\left(X_{t^{\prime}}\right)\right] .
$$

Der ATT wird auf Basis des beobachteten Ergebnisses $(y)$, des Teilnahmestatus $(d)$ und der betrieblichen Merkmale $(x)$ für die Teilnehmer $i\left\{y_{i}, d_{i}, x_{i}\right\}_{i=1: N, D=1}$ und Nichtteilnehmer $j\left\{y_{j}, d_{j}, x_{j}\right\}_{j=1: N, D=0}$ in der Stichprobe der Buchführungsdaten geschätzt.

\subsubsection{Matching-Algorithmen und Matching-Schätzer}

Der Matching-Algorithmus beschreibt den Prozess der Kontrollgruppenbildung, während der Matching-Schätzer die Bedingungen definiert, entsprechend denen Teilnehmer und Nichtteilnehmer einander zugeordnet werden.

Als Standardverfahren wird in der empirischen Analyse ein Greedy Algorithmus ${ }^{36}$ mit einem 1:1 Caliper Schätzer ohne Wiederholung verwendet (PARSON, 2001). Der Caliper

Schätzer $\left(\alpha_{\text {Caliper }}\right)$ geht zurück auf COCHRAN und RUBIN (1973) und ist formal beschrieben als

Alternative Matching-Algorithmen sind das optimale Matching (RosenbaUM, 1989) und das vollständige Matching (HANSEN, 2004; RosenBAuM, 1991). 


$$
\alpha_{\text {Caliper }}=\left\|p\left(x_{i t^{\prime}}\right)-p\left(x_{j t^{\prime}}\right)\right\|<\delta \quad \text { mit } \delta(0,00001, \ldots, 0,1),
$$

wobei $\delta$ der Caliper ist, der die maximal zulässige Differenz zwischen dem Propensity Score eines Teilnehmers, $p\left(x_{i t^{\prime}}\right)$, und dem eines Kontrollbetriebes, $p\left(x_{j t^{\prime}}\right)$, definiert. Der Caliper verhindert die Zuordnung von Teilnehmer- und Kontrollbetrieben mit zu unterschiedlichen Merkmalen. Teilnehmerbetriebe, für die kein Kontrollbetrieb innerhalb des definierten Calipers gefunden wird, scheiden für die weitere Analyse aus.

Der verwendete Greedy Algorithmus beruht auf einem variablen Caliper: Im ersten Schritt werden jene Kontrollbetriebe und Teilnehmer einander zugeordnet, die einen bis auf fünf Nachkommastellen ( $\delta=0,00001)$ identischen Propensity Score aufweisen. In den folgenden Schritten wird der Propensity Score bis auf eine Nachkommastelle $(\delta=0,1)$ aufgerundet und das Matching wiederholt. ${ }^{37}$ Im ersten Schritt werden wenige, aber sehr ähnliche Kontrollpaare gebildet. In den folgenden Schritten nimmt die Anzahl der Kontrollpaare zu, die Ähnlichkeit zwischen diesen jedoch ab. Jedem Teilnehmer wird ein Kontrollbetrieb zugeordnet (1:1 Matching). Die Zuordnung erfolgt ohne Wiederholung, d. h. ein Kontrollbetrieb kann nur einmal ausgewählt werden und steht zur Bildung weiterer Kontrollpaare nicht mehr zur Verfügung.

Neben dem verwendeten Caliper Schätzer gibt es eine Vielzahl weiterer Möglichkeiten, die Zuordnung von Teilnehmern und Nichtteilnehmern vorzunehmen. Einen Überblick über verschiedene Matching-Schätzer geben BECKER und ICHINO (2002), LEUVEN und SiAnesi (2003), REINOWSKI (2006), HeCKMAN et al. (1998) sowie Smith und TodD (2005). Da sich bisher kein Matching-Schätzer als generell überlegen herausgestellt hat (BASER, 2006; Gu und Rosenbaum, 1993), werden die Ergebnisses des Greedy Matching mit den Ergebnissen zweier alternativer Verfahren verglichen: Mit einem modifizierten Caliper Schätzer (fixer 1:1 Caliper Schätzer mit $\delta=0,00001$, mit Wiederholung) und einem Kernel Schätzer (HECKMAN et al., 1997; 1998).

Der modifizierte Caliper Schätzer unterscheidet sich in zwei Punkten vom Ersteren: Zum einen ist der Caliper konstant. Zum anderen erfolgt das Matching mit Wiederholung. Matching mit Wiederholung kann bei nur wenigen potenziellen Kontrollbetrieben eine Lösung sein. Ein Risiko hierbei ist, dass ein und dieselben Nichtteilnehmer sehr häufig als Kontrollbetriebe selektiert werden und damit die Varianz in der Stichprobe reduziert wird.

37 Für AUM-Teilnehmer, Deutschland gesamt (vgl. Tabelle 8.2), werden die Caliper 0,00001 bis 0,0001 verwendet, da ansonsten die beobachteten Unterschiede zwischen Teilnehmern und Kontrollbetrieben nicht vollständig kontrolliert werden können. 
Beim Kernel Schätzer wird ein Teil der Nichtteilnehmerbeobachtungen zur Konstruktion eines hypothetischen Kontrollbetriebes verwendet. Die Gewichtung $w(i, j)$ der einzelnen Kontrollbetriebe wird entsprechend der Nähe zur Teilnehmerbeobachtung vorgenommen,

$$
w(i, j)=\frac{k_{i j}}{\sum_{k \in(d=0)} k_{i k}} \text { mit, } k_{i k}=k\left(\frac{p\left(x_{k t^{\prime}}\right)-p\left(x_{i t^{\prime}}\right)}{h}\right)
$$

wobei $k(\cdot)$ die Kernfunktion, $h$ die Bandbreite und $p\left(x_{k t^{\prime}}\right)$ der Propensity Score des hypothetischen Kontrollbetriebes ist. Die Bandbreite bestimmt das Intervall der zu verwendenden Nichtteilnehmerbeobachtungen bei der Schätzung des hypothetischen Kontrollbetriebes. In der empirischen Analyse wird eine Gaußsche Kernfunktion mit optimaler Bandbreite $h$ verwendet (vgl. RAMOS, 2005). ${ }^{38}$

\section{Vergleich verschiedener Matching-Schätzer}

Die Effizienz eines Schätzers zeigt sich darin, inwieweit durch diesen die beobachteten Unterschiede zwischen der Teilnehmer- und Kontrollgruppe beseitigt werden. Die Effizienz verschiedener Matching-Schätzer ist Gegenstand zahlreicher Studien (BASER, 2006; Dehejia und Wahba, 2002; Gu und Rosenbaum, 1993; Smith und TodD, 2005). Keines der untersuchten Verfahren stellte sich als generell überlegen heraus. Daher ist die Effizienz des Schätzers für jeden Einzelfall zu überprüfen.

BASER (2006) schlägt hierfür unter anderem den T-Test bzw. Chi-Quadrat-Test sowie den standardisierten Bias (SB) und die prozentuale Verringerung des Bias (PVB) vor. Der SB ist definiert als

$$
S B=\frac{100 \times\left(\bar{x}_{i t^{\prime}}-\bar{x}_{j t^{\prime}}\right)}{0,5 \times\left(s_{\bar{x}_{i t^{\prime}}}+s_{\bar{x}_{j t^{\prime}}}\right)}
$$

wobei $\bar{x}_{i t^{\prime}}$ und $\bar{x}_{j t^{\prime}}$ die Mittelwerte der im Logit-Modell verwendeten Kovariate von Teilnehmern- und Kontrollbetrieben und $s_{\bar{x}_{i t^{\prime}}}$ bzw. $s_{\bar{x}_{j t^{\prime}}}$ die Standardabweichungen dieser Kovariate sind (LECHNER, 2002). Der $S B$ kann interpretiert werden als der Bias in Prozent der mittleren Standardabweichung. Die prozentuale Verringerung des Bias (SB reduziert) wird berechnet als

$$
S B \text { reduziert }=\frac{\left(\bar{x}_{s i t^{\prime}}-\bar{x}_{s j t^{\prime}}\right)-\left(\bar{x}_{a i t^{\prime}}-\bar{x}_{a j t^{\prime}}\right)}{\bar{x}_{a i t^{\prime}}-\bar{x}_{a j t^{\prime}}} \times 100
$$

\footnotetext{
38 Die optimale Bandbreite wird auf Basis des Mittleren Integrierten Quadratischen Fehlers (MISE) bestimmt.
} 
wobei $\bar{x}_{a i t^{\prime}}$ bzw. $\bar{x}_{a j t^{\prime}}$ die Mittelwerte der Kovariate aller $(a)$ Teilnehmer- und Kontrollbetriebe vor Durchführung des Programms und $\bar{x}_{s i t^{\prime}}$ bzw. $\bar{x}_{s j t^{\prime}}$ die Mittelwerte der Kovariate der durch das Matching selektierten $(s)$ Teilnehmer- und Kontrollbetriebe vor Durchführung des Programms bezeichnen (BASER, 2006).

Da sich die praktische Relevanz des Programmeffektes nicht aus seiner statistischen Signifikanz ergibt, wird zusätzlich die Effektstärke $d$ nach COHEN (1988) berechnet. Die Effektstärke entspricht der Differenz der mittleren Ergebnisänderung in Teilnehmer- und Kontrollbetrieben geteilt durch die gepoolte Standardabweichung beider Teilstichproben. Eine Effektstärke $<0,2$ gilt als klein, zwischen 0,2 und unter 0,5 als mittel und $>0,8$ als groß.

\subsubsection{Analyse heterogener Programmeffekte}

Mit Hilfe des in Kapitel 7.1.1 dargestellten Standardmodells wird der über alle Teilnehmer gemittelte Effekt der Programmteilnahme geschätzt. Die theoretischen Überlegungen in Kapitel 3 legen nahe, dass sich der Effekt der Programmteilnahme zwischen Betrieben mit unterschiedlichen Merkmalen unterscheidet. Um die Heterogenität des Programmeffektes in Abhängigkeit von regionalen und betrieblichen Merkmalen zu analysieren, werden zwei Ansätze (nicht-parametrische Regressionen, stratifiziertes Matching) verwendet. Beide Ansätze bauen auf dem bereits vorgestellten Standardmodell auf.

\section{Nicht-parametrische Regressionen}

In der empirischen Analyse wird z. B. untersucht, ob das betriebliche Flächenwachstum von Teilnehmern und Nichtteilnehmern im Zeitraum 2000 bis 2005 in Abhängigkeit von der Teilnahmewahrscheinlichkeit eines Betriebes variiert. Hierzu wird eine nichtparametrische Regressionsanalyse im Anschluss an das vorgestellte Standardmodell durchgeführt. Für jede Ergebnisvariable wird - getrennt für Teilnehmer und Nichtteilnehmer - ein nicht-parametrisches Regressionsmodell geschätzt,

$$
\begin{aligned}
& \Delta y_{i}^{1}=f\left(g_{i t^{\prime}}, v_{i t^{\prime}}\right) \\
& \Delta y_{j}^{0}=f\left(g_{j t^{\prime}}, v_{j t^{\prime}}\right)
\end{aligned}
$$

wobei $\Delta y_{i}^{1}$ und $\Delta y_{j}^{0}$ die beobachteten Ergebnisänderungen der Teilnehmer und der Kontrollbetriebe im Zeitraum von 2000 bis 2005 sind, $g_{i t^{\prime}}$ und $g_{j t^{\prime}}$ das untersuchte betriebliche Merkmal und $v_{i t^{\prime}}$ und $v_{j t^{\prime}}$ die zufälligen Fehler mit einem Erwartungswert von Null. Der funktionale Zusammenhang $(f)$ zwischen der abhängigen und der unabhängigen Variable wird nicht spezifiziert, um einen flexiblen Zusammenhang zwischen beiden Parametern zu erlauben. Für die Schätzung wird ein nicht-parametrischer Kernel Schätzer (Nadaraya-Watson Schätzer) verwendet (LECHNER, 2002). Die geschätzte Ergebnisände- 
rung wird für Teilnehmer und Nichtteilnehmer graphisch dargestellt und visuell interpretiert.

Eine Voraussetzung für die Anwendung nicht-parametrischer Regressionen im Anschluss an die Matching-Analyse ist die Common Support Condition. In Kapitel 8.2 wird gezeigt, dass diese Bedingung nicht erfüllt ist und die Regressionsschätzung daher nicht für jedes stetig skalierte Merkmal eingesetzt werden kann (vgl. Kapitel 8.2, Überprüfung der Modellannahmen).

\section{Stratifiziertes Matching}

Das stratifizierte Matching wird verwendet, wenn die beobachtete Ergebnisänderung in Abhängigkeit von einem diskreten Merkmal, z. B. der Betriebsform, analysiert wird. Hierzu werden die Teilnehmerbetriebe vor dem Matching, entsprechend ihrer Betriebsform, in $n$ geschichtete Stichproben unterteilt $\left(g_{i t^{\prime}}^{n}\right)$. Der Programmeffekt wird für jede Stichprobe nach Gleichung (7.4) ermittelt.

Für die Matching-Analyse wird das SAS-Makro Greedy Matching (PARSON, 2001) und das Stata Programm Psmatch2 (LeUven und SiAnesi, 2003) verwendet. Die nichtparametrischen Regressionsschätzungen werden in Stata mit Hilfe des Programms Kernregl (RAmos, 2005) durchgeführt.

\subsection{Regressionsmodelle für Paneldaten}

Anders als im Matching-Modell wird in Regressionsmodellen ein - zumeist additivlinearer - funktionaler Zusammenhang zwischen der abhängigen Variablen und deren Determinanten (Programmteilnahme, Kontrollvariablen) unterstellt. Die Ursache des Selektionseffektes kann in Regressionsmodellen als ein Problem einer unvollständigen Modellspezifikation interpretiert werden: Nicht alle Faktoren, die die Programmteilnahme und das interessierende Ergebnis beeinflussen, sind im Modell berücksichtigt, da diese unbeobachtet, unbekannt, nicht oder schwer messbar sind. Die Annahme, dass alle relevanten Faktoren durch das spezifizierte Modell kontrolliert werden, liegt jedem Regressionsmodell zu Grunde. Insofern erfordert die Korrektur des Selektionseffektes in parametrischen Verfahren ein besonderes Augenmerk auf die Einhaltung der ohnehin unterstellten Annahme der Vollständigkeit des Modells.

Auf unterschiedlichen Annahmen beruhende Regressionsmodelle führen in der Regel zu unterschiedlichen Ergebnissen. Häufig kann nicht a priori entschieden werden, welche Modellspezifikation für die jeweilige Fragestellung die geeignete ist. Aus diesem Grund werden mehrere Standardmodelle spezifiziert (gepooltes OLS-Modell, Fixed-EffektModell, dynamische GMM-Modelle). Diese Modelle werden in Kapitel 9 hinsichtlich 
ihrer Annahmen und Ergebnisse verglichen. Zur Analyse heterogener Programmeffekte werden diese Standardmodelle um Interaktionsterme erweitert bzw. zu einem Selektionsmodell modifiziert.

\subsubsection{Verwendete Modelle}

\subsubsection{Gepooltes Panelmodel mit OLS-Schätzer}

Im gepoolten OLS-Modell (POLS) werden die Beobachtungen aller zur Verfügung stehenden Untersuchungszeitpunkte gleichzeitig analysiert. Das verwendete POLS hat die allgemeine Form

$$
y_{i t}=\gamma w_{i t}+\beta x_{i t}+u_{i t}, \quad i=1, \ldots, N, t=1, \ldots, T,
$$

wobei $y_{i t}$ das zu erklärende Ergebnis des Betriebes $i$ zum Zeitpunkt $t$ ist. Die erklärenden Variablen werden in den $1 \times K$ bzw. $1 \times G$ Vektoren $w_{i t}$ und $x_{i t}$ zusammengefasst. Elemente in $w_{i t}$ können endogen sein und umfassen gegenwärtige und frühere Werte der Kontrollvariablen $\left(z_{i t}\right)$, die Programmteilnahme $\left(d_{i t}\right)$ sowie Lags der abhängigen Variable $\left(w_{i t}=\left(z_{i t}, z_{i, t-1}, d_{i t}, y_{i, t-1}, y_{i, t-2}\right)\right)$. Der Vektor $x_{i t}$ beinhaltet ausschließlich exogene Variablen inklusive jahresspezifischer Dummies $\left(\mu_{t}\right)$ und regionsspezifischer Dummies $\left(c_{r}\right)$. Alle Elemente in $w_{i t}$ und $x_{i t}$ können zeitkonstant oder zeitvariabel sein. Die zu schätzenden Koeffizienten werden durch die $k \times 1$ bzw. $g \times 1$ Vektoren $\gamma$ bzw. $\beta$ repräsentiert. Der Fehlerterm des Modells $\left(u_{i t} \equiv \eta_{i}+v_{i t}\right)$ setzt sich aus dem unbeobachteten, individuellen Fehler $\left(\eta_{i}\right)$ und dem zufälligen betriebs- und zeitspezifischen Fehler $\left(v_{i t}\right)$ zusammen.

Modell (7.10) berücksichtigt explizit die Möglichkeit einer dynamischen Modellspezifikation. Durch die Verwendung von Lags der abhängigen Variablen als Regressoren wird die Historie des Ergebnisses, z. B. die betriebliche LF des Vorjahres, explizit berücksichtigt. Da die betriebliche LF eine geringe zeitliche Varianz aufweist, hat die betriebliche Flächenausstattung des Vorjahres einen hohen Erklärungsgehalt für die Flächenausstattung des Folgejahres.

Endogene Regressoren, zusammengefasst im Vektor $w_{i t}$, weisen eine Korrelation mit dem Fehlerterm des Modells $\left(u_{i t}\right)$ auf. Ursachen für die Endogenität können sein: simultane Kausalität, im Modell nicht kontrollierte (unbeobachtete) Faktoren und Messfehler ${ }^{39}$ (WoOLdRIDGe, 2002: $50 \mathrm{f}$.). 
Simultane Kausalität: Betriebliche Entscheidungen hinsichtlich des Faktoreinsatzes werden häufig simultan getroffen und wirken sich direkt auf die Höhe flächengebundener Direktzahlungen an den Betrieb aus. Die genannten Variablen werden in der empirischen Modellspezifikation berücksichtigt und bedingen sich teilweise gegenseitig. So haben z. B. Direktzahlungen häufig einen positiven Einfluss auf das betriebliche Flächenwachstum. Die Höhe der Direktzahlungen ist aber unter anderem von der betrieblichen LF abhängig. Durch die Verwendung von Lags endogener Regressoren kann die durch Simultanität verursachte Endogenität weitgehend berücksichtigt werden.

Unbeobachtete Faktoren: In einem Modell sind in der Regel nicht alle relevanten Einflussfaktoren auf das interessierende Ergebnis bzw. die Entscheidung zur Programmteilnahme berücksichtigt. Unbeobachtete, jahresspezifische Effekte werden im Modell (7.10) durch entsprechende Jahresdummies $\left(\mu_{t}\right)$ kontrolliert. Unbeobachtete, betriebsspezifische Faktoren werden nicht kontrolliert. Der Einfluss unbeobachteter Faktoren auf das Ergebnis schlägt sich teilweise im Fehlerterm nieder $\left(u_{i t} \equiv \eta_{i}+v_{i t}\right)$ und, im Fall der Korrelation mit beobachteten Faktoren $\left(w_{i t}\right)$, teilweise in den geschätzten Koeffizienten der mit $\eta_{i}$ korrelierenden Regressoren.

Für das POLS gelten die folgenden Standardannahmen (WoOLDRIDGE, 2002: 171):

- A1: Die zu schätzenden Koeffizienten $(\gamma, \beta)$ sind für alle Beobachtungen konstant.

- A2: Der Fehler hat einen Erwartungswert von Null und weist keine Korrelation mit den Regressoren des gleichen Zeitpunktes $t$ auf $\left(E\left(u_{i t} \mid w_{i t}, x_{i t}\right)=0\right)$.

- A3: Der Fehler weist für alle Zeitpunkte und Betriebe eine homogene Varianz $\left(\operatorname{Var}\left(u_{i t}\right)=\sigma_{u}^{2}\right)$ und keine Autokorrelation zwischen verschiedenen Zeitpunkten $\left(\operatorname{Cov}\left(u_{i t}, u_{i s}\right)=0\right.$ für $\left.t \neq s\right)$ und Betrieben $\left(\operatorname{Cov}\left(u_{i t}, u_{j t}\right)=0\right.$ für $\left.i \neq j\right)$ auf.

A1 impliziert, dass der mittlere Effekt der Programmteilnahme (ATE) und der mittlere Effekt der Programmteilnahme auf die Teilnehmer (ATT) gleich sind (WoOLDRIDGE, 2002: 611).

A2 und A3 münden in die Annahme eines iid-verteilten Fehlers. A2 umfasst die Annahme der Exogenität aller Regressoren (vgl. Diskussion oben). Ist die Endogenität auf simultane Kausalität zurückzuführen, so kann die Verwendung von Lags eine Lösung sein. Unbeobachtete, betriebsspezifische Faktoren schlagen sich im Fehlerterm nieder und verursachen eine Korrelation zwischen einem oder mehreren Regressoren und dem Fehlerterm. Eine Verletzung von A2 ist im POLS daher wahrscheinlich. 
A3 betrifft die Fehlerstruktur des Modells. Gegenüber Heteroskedastizität und Autokorrelation robuste Standardfehler werden durch die Verwendung robuster Varianzschätzer ${ }^{40}$ erreicht. In der dynamischen Modellspezifikation kann die Autokorrelation der Fehler durch die Verwendung genügend vieler Lags der abhängigen und erklärenden Variablen reduziert werden (dynamische Vollständigkeit des Modells, vgl. WoOLDRIDGE, 2002: 173).

Unter den diskutierten Annahmen hat die Verletzung von A2 vermutlich weit reichende Konsequenzen. Nicht messbare oder unbeobachtete Faktoren dürften einen erheblichen Einfluss auf die betriebliche Faktorausstattung haben und können zu verzerrten Schätzern führen. Das im Folgenden beschriebene Fixed-Effekt Modell (FE-Modell) zeigt einen methodischen Ansatz zur Kontrolle betriebsspezifischer, unbeobachteter Effekte.

\subsubsection{Fixed-Effekt-Modell}

Der Einfluss unbeobachteter, individueller Effekte auf des Ergebnis kann, unter bestimmten Annahmen, mit Hilfe eines FE-Modell kontrolliert werden (WoOLDRIDGE, 2002: 265 ff., 300 ff.). Das verwendete FE-Modell hat die Form

$$
y_{i t}=\gamma w_{i t}+\beta x_{i t}+\eta_{i}+v_{i t}, \quad i=1, \ldots, N, t=1, \ldots, T,
$$

wobei $y_{i t}, w_{i t}, x_{i t}$ sowie die zu schätzenden Koeffizienten $\gamma$ und $\beta$ wie im POLS definiert sind. Der betriebsspezifische Effekt wird durch $\eta_{i}$ repräsentiert und kann willkürlich mit Elementen in $x_{i t}$ und $w_{i t}$ korrelieren. Der Fehlerterm des Modells setzt sich aus dem fixen Effekt und dem betriebs- und zeitspezifischen Effekt zusammen $\left(u_{i t} \equiv \eta_{i}+v_{i t}\right)$.

Um den betriebsspezifischen Effekt $\left(\eta_{i}\right)$ zu kontrollieren, werden die Daten einer within Transformation unterzogen. Der über alle Untersuchungsjahre $t$ gebildete betriebsspezifische Mittelwert der Variablen $\left(\bar{y}_{i}, \bar{w}_{i}, \bar{x}_{i}, \bar{\eta}_{i}\right)$ wird vom jahresspezifischen Wert der Variablen $\left(y_{i t}, w_{i t}, \mathbf{x}_{i t}, \eta_{i t}\right)$ subtrahiert, so dass

$$
\left(y_{i t}-\bar{y}_{i .}\right)=\left(x_{i t}-\bar{x}_{i}\right) \beta+\left(d_{i t}-\bar{d}_{i .}\right) \delta+\left(\eta_{i}-\bar{\eta}_{i}\right)+\left(v_{i t}-\bar{v}_{i .}\right), i=1, \ldots, N, t=1, \ldots, T .
$$

Der betriebsspezifische Effekt wird ausdifferenziert, da $\eta_{i}-\bar{\eta}_{i}=0$ ist. Bei Verwendung within-tranformierter Daten kann der Effekt der Programmteilnahme nur für Betriebe geschätzt werden, deren Teilnahmestatus im Untersuchungszeitraum variiert. Im Unterschied 
zum POLS sind im FE-Modell daher auch keine zeitkonstanten Regressoren, wie z. B. regionsspezifische Dummies, im Vektor $x_{i t}$ enthalten. $^{41}$

Für die Schätzung der transformierten Modellgleichung (7.12) wird ein OLS-Schätzer verwendet. Die Annahmen des FE-Modells sind denen des POLS daher sehr ähnlich (WOOLDRIDGE, 2002: 269, 298):

- A1: Der Fehler hat, kontrolliert für $\eta_{i}$, einen Erwartungswert von Null und korreliert nicht mit gegenwärtigen, zukünftigen und vergangenen Werten der Regressoren $\left(E\left(v_{i t} \mid w_{i}, x_{i}, \eta_{i}\right)=0\right)$.

- A2: Der Fehler hat eine konstante Varianz für alle $t$ und ist nicht autokorreliert $\left(E\left(v_{i} v_{i}^{\prime} \mid w_{i}, x_{i}, \eta_{i}\right)=\sigma_{v}^{2} I_{T}\right)$.

A1 beinhaltet zwei Implikationen: Zum einen wird durch $\eta_{i}$ nur der Einfluss zeitkonstanter Faktoren kontrolliert (z. B. natürliche Standortbedingungen), die additiv separabel auf das Ergebnis wirken ${ }^{42}$ (WOOLDRIDGE, 2002: 638). In der Realität beeinflussen vermutlich auch zeitvariable, unbeobachtete Faktoren die betriebliche Faktorausstattung bzw. die Entscheidung zur Programmteilnahme (z. B. familiäre Veränderungen). Weiterhin ist zu erwarten, dass der Effekt der Programmteilnahme nicht additiv separabel ist, sondern von (un-)beobachteten betrieblichen und regionalen Faktoren abhängt.

Zum anderen wird die Annahme der Exogenität auf alle früheren und zukünftigen Realisationen der Regressoren ausgedehnt (strikte Exogenität). Infolge der within Transformation gehen frühere $\left(w_{i, t-s}, x_{i, t-s}\right)$ und zukünftige $\left(w_{i, t+s}, x_{i, t+s}\right)$ Realisationen in den jeweils gegenwärtigen Wert des Regressors $\left(w_{i t}, x_{i t}\right)$ ein. Die Annahme strikter Exogenität bezieht sich daher auf die Regressoren aller Untersuchungsjahre $\left(w_{i}, x_{i}\right)$. CHAMBERLAIN (1982) führt hierfür die Terminologie strict exogeneity conditional on the unobserved effect ein.

Der Einfluss der Programmteilnahme auf die Veränderung des betrieblichen Faktoreinsatzes im Untersuchungszeitraum kann durch ein statisches ökonometrisches Modell nicht adäquat abgebildet werden. Die Annahme strikter Exogenität schließt z. B. eine wünschenswerte dynamische Spezifikation des FE-Modells aus (WoOLDRIDGE, 2002: 256). Für Modell (7.12) wird daher die weniger restriktive Annahme der sequential exogeneity conditional on the unobserved effect gewählt (CHAMBERLAIN, 1992). Kontrolliert für $\eta_{i}$, kann der Fehler $v_{i t}$ mit zukünftigen Realisationen der Regressoren $\left(w_{i, t+1}, \ldots, w_{i T}\right.$, $\left.x_{i, t+1}, \ldots, x_{i T}\right)$ korrelieren, jedoch nicht mit deren gegenwärtigen und vergangenen Realisa-

41 Die Berücksichtigung zeitkonstanter Regressoren ist in Form von Interaktionstermen mit zeitvariablen Regressoren (z. B. Jahresdummies) möglich.

42 Additive Separabilität unterstellt, dass der Einfluss des unbeobachteten Effektes auf das interessierende Ergebnis nicht vom Niveau anderer Faktoren abhängt. 
tionen $\left(w_{i t}, w_{i, t-1}, \ldots, w_{i 1}, x_{i t}, x_{i, t-1}, \ldots, x_{i 1}\right)$. Erst die Annahme sequentieller Exogenität ermöglicht eine dynamische Modellspezifikation und die Verwendung von Lags von Regressoren, die zum Zeitpunkt $t$ endogen sind, nicht jedoch zum Zeitpunkt $t-1$.

Durch die Annahme sequentieller Exogenität kann die durch endogene Regressoren verursachte Autokorrelation des Fehlers in der Regel nicht vollständig verhindert werden (WoOldRIDGE, 2002: 301). ${ }^{43}$ Daher werden gegenüber Heteroskedastizität und Autokorrelation robuste Varianzschätzer verwendet.

Im Vergleich zum POLS kann durch das FE-Modell der Einfluss unbeobachteter, zeitkonstanter Faktoren teilweise kontrolliert werden. Der Preis hierfür sind ungleich strengere Annahmen hinsichtlich der Exogenität der Regressoren. Viele potenzielle Determinanten der betrieblichen Faktorausstattung bzw. der Programmteilnahme sind aufgrund der Simultanität betrieblicher Entscheidungen vermutlich endogen. Ein Standardansatz zum Umgang mit endogenen Regressoren ist deren Instrumentierung durch exogene zusätzliche Variablen. Da viele potenzielle Instrumente auf einzelbetrieblicher Ebene ebenfalls endogen sind, ist das Auffinden von geeigneten Instrumenten (korreliert mit dem endogenen Regressor) und validen Instrumenten (nicht korreliert mit der zu erklärenden Variablen) mitunter schwierig.

Als Schlussfolgerung der bisher angestellten Überlegungen sollte ein zu präferierendes Modell folgende Anforderungen erfüllen: i) die Kontrolle unbeobachteter Effekte, ii) eine dynamische Modellspezifikation, iii) die Instrumentierung (mehrerer) endogener Regressoren auch beim Fehlen zusätzlicher (externer) Instrumente und iv) eine gegenüber Heteroskedastizität und Autokorrelation robuste Varianzschätzung. Dynamische GMMModelle können, unter bestimmten Annahmen, diese Anforderungen erfüllen und werden im Folgenden vorgestellt.

\subsubsection{Dynamische GMM-Modelle}

Arrelano und Bond (1991), Arellano und Bover (1995) sowie Blundell und Bond (1998) entwickelten verschiedene Schätzer für dynamische Modelle. Die Autoren bauen dabei auf früheren Arbeiten von ANDERSON und HSIAO (1982) sowie Holtz-EAKIN et al. (1988) auf. Das verwendete autoregressive GMM-Modell zweiter Ordnung (AR2) gleicht in seiner allgemeinen Form dem FE-Modell in Gleichung (7.11)

43 Um die Inkonsistenz des dynamischen FE-Modells zu begrenzen, muss die Summe der Schätzer für $y_{i, t-1}$ und $y_{i, t-2}$ kleiner eins sein (WOOLDRIDGE, 2002: 302). Die hierdurch implizierte Annahme der Stationarität der Daten wird im Zusammenhang mit den unter 7.2.1.3 vorgestellten GMM-Modellen diskutiert. 


$$
y_{i t}=\gamma w_{i t}+\beta x_{i t}+\eta_{i}+v_{i t}, \quad i=1, \ldots, N, t=1, \ldots, T
$$

wobei $y_{i t}$ das zu erklärende Ergebnis, $w_{i t}$ ein $1 \times K$ Vektor mit gegenwärtigen und früheren Werten endogener oder vordeterminierter ${ }^{44}$ Regressoren $\left(w_{i t}=\left(z_{i t}, z_{i, t-1}, d_{i t}, y_{i, t-1}, y_{i, t-2}\right)\right)$ und $x_{i t}$ ein $1 \times G$ Vektor strikt exogener Regressoren ist. Die Regressoren in $w_{i t}$ und $x_{i t}$ sind ausschließlich zeitlich variabel. Die zu schätzenden Koeffizienten $\gamma$ und $\beta$ sind $k \times 1$ und $g \times 1$ Vektoren. Der zusammengesetzte Fehlerterm hat die Form $u_{i t} \equiv \eta_{i}+v_{i t}$.

Um den Einfluss betriebsspezifischer Faktoren zu kontrollieren, werden die orthogonalen Abweichungen (OR) ${ }^{45}$ aller Modellvariablen gebildet (ARELLANO und BOVER, 1995). Hierfür wird der Mittelwert aller verbleibenden zukünftigen Werte einer Variablen vom gegenwärtigen Wert dieser Variable subtrahiert. Dargestellt am Beispiel der abhängigen Variable $y_{i t}$ sind die orthogonalen Abweichungen definiert als

$$
y_{i t}^{\perp}=c_{t}\left(y_{i t}-\frac{1}{T-t} \sum_{s>t} y_{i s}\right), \quad i=1, \ldots, N, t=1, \ldots, T-1
$$

wobei $c_{t}$ mit $c_{t}=\sqrt{(T-t) /(T-t+1)}$ ein Gewichtungsfaktor zur Angleichung der Varianz, $(T-t)$ die Anzahl verbleibender Beobachtungszeitpunkte, $y_{i s}$ die verbleibenden, zukünftigen Werte des Merkmals und $y_{i t}$ die gegenwärtige Ausprägung der Variable sind. Orthogonale Abweichungen haben gegenüber der within Transformation den Vorteil, dass die Endogenität einer Variablen nicht auf alle zukünftigen Werte übertragen wird. Das in orthogonale Abweichungen transformierte Modell aus Gleichung (7.13), im Folgenden als GMM-OR bezeichnet, hat die Form

$$
y_{i t}^{\perp}=\gamma w_{i t}^{\perp}+\beta x_{i t}^{\perp}+\eta_{i}^{\perp}+v_{i t}^{\perp}, \quad i=1, \ldots, N, t=1, \ldots, T-1,
$$

wobei der betriebsspezifische Effekt durch die OR Transformation kontrolliert wird, da $\eta_{i}^{\perp}=0$. Um die Korrelation zwischen dem Fehlerterm und Elementen in $w_{i t}$ zu kontrollieren, werden frühere untransformierte Lags der endogenen bzw. vordeterminierten Variablen als Instrumente genutzt. Unter der Annahme nicht autokorrelierter Fehler (A1) können endogene Regressoren in $w_{i t}^{\perp}$ durch $w_{i, t-2}$ und frühere $\operatorname{Lags}\left(w_{i, t-2}, \ldots, w_{i, T-2}\right)$ instrumentiert werden und vordeterminierte Regressoren in $w_{i t}^{\perp}$ durch $w_{i, t-1}$ oder frühere Lags $\left(w_{i, t-1}, \ldots, w_{i, T-1}\right)$. Ist ein Regressor strikt exogen, stehen alle früheren, gegenwärtigen und zukünftigen Werte $\left(x_{i}\right)$ als Instrument für $w_{i t}^{\perp}$ zur Verfügung.

\footnotetext{
44 Vordeterminierte Regressoren (z. B. $y_{i, t-1}$ ) sind potenziell korreliert mit früheren Werten des Fehlerterms.

45 Orthogonale Abweichungen haben gegenüber den standardmäßig verwendeten ersten Differenzen (first-differences) den Vorteil, dass die Anzahl fehlender Werte reduziert wird, da ein fehlender untransformierter Wert nicht automatisch einen fehlenden transformierten Wert zur Folge hat.
} 
Das GMM-OR beruht auf folgenden Annahmen (vgl. BLunDELL und BOND, 1998):

- A1. Der Erwartungswert des Fehlerterms, $v_{i t}$, und des individuellen Effektes, $\eta_{i}$, ist Null $\left(E\left(v_{i t}\right)=0, E\left(\eta_{i}\right)=0, E\left(v_{i t} \eta_{i}\right)=0\right)$. Der Fehlerterm ist nicht autokorreliert $\left(E\left(v_{i t} v_{i s}\right)=0\right.$ für $\left.s \neq t\right)$.

- A2. Die Anfangsbedingungen, $y_{i 1}$, sind (exogen) vordeterminiert und korrelieren nicht mit dem Fehler $v_{i t}$ der folgenden Jahre $\left(E\left(y_{i 1} v_{i t}\right)=0\right)$.

- A3. Modell (7.15) gilt für den Fall, dass der zu untersuchende Prozess dynamisch stabil ist, d. h. die Summe der Koeffizienten von $y_{i, t-1}$ und $y_{i, t-2}$ einen absoluten Wert kleiner eins annimmt $\left|\alpha_{1}+\alpha_{2}\right|<1$ (Annahme der Stationarität).

Eine Schwäche des GMM-OR ist, dass die Lags endogener oder vordeterminierter Regressoren schlechte Instrumente für deren transformierten Werte sein können. Dies ist der Fall, wenn Modellvariablen eine hohe zeitliche Persistenz aufweisen oder die Varianz des fixen Effektes im Vergleich zum Fehler sehr hoch ist (BLundELL und Bond, 1998). In dieser Situation sind die Schätzergebnisse des GMM-OR nach unten verzerrt. Die Effizienz des GMM-OR kann durch die Verwendung weiterer Instrumente verbessert werden, allerdings nur in Kombination mit der zusätzlichen Annahme A4 (ersetzt A2 des GMM-OR).

- A4. Der Anfangswert der zu erklärenden Variable, $y_{i 1}$, ist nicht exogen fixiert, sondern stellt eine zufällige Abweichung vom langfristigen Gleichgewichtswert $\left(\eta_{i} / 1-\alpha\right)$ eines Betriebes $y_{i}$ dar. Der Grad der Abweichung vom Anfangswert, $u_{i 1}$, darf nicht mit dem Niveau des Gleichgewichtswertes und damit nicht mit $\eta_{i}$ korrelieren $\left(E\left(u_{i 1} \eta_{i}=0\right)\right)$ (BLUNDELL und BOND, 1998).

A4 impliziert, dass der Mittelwert der abhängigen Variable eines Panels, $\bar{y}_{i}$, konstant ist und Abweichungen um diesen Mittelwert nur zufällig sind. Diese Annahme ist, bezogen auf betriebliche Faktorausstattung, nicht immer haltbar. So unterliegt z. B. der betriebliche Flächen- und Kapitaleinsatz im Mittel einem positiven Entwicklungstrend. Eine weniger starke Annahme ist, dass der Entwicklungstrend in den analysierten Betrieben ein vergleichbares Niveau hat. Da das verwendete Modell zeitspezifische Dummyvariablen enthält und die Daten transformiert werden, ist es ausreichend, wenn die mittlere Abweichung vom Trend konstant ist (BLUNDELL und BOND, 2000).

Unter den Annahmen A1 und A4 können endogene Regressoren in $w_{i t}$ durch $w_{i, t-1}^{\perp}, \ldots, w_{i, T-1}^{\perp}$ und vordeterminierte Regressoren in $w_{i t}$ durch $w_{i t}^{\perp}, \ldots, w_{i T}^{\perp}$ bzw. $y_{i, t-1}^{\perp}, . ., y_{i, T-1}^{\perp}$ instrumentiert werden. Für exogene Regressoren stehen wiederum alle früheren, gegenwärtigen und zukünftigen transformierten Werte als Instrumente zur Verfügung $\left(x_{i}^{\perp}\right)$. Dieses von BLUNDELL und BOND (1998) vorgeschlagene System GMM-Modell, im Folgenden GMMSYS, beruht auf der simultanen Schätzung der transformierten (7.15) und der untransformierten Gleichung (7.13) und kann zu weniger verzerrten Schätzergebnissen führen. Die 
zusätzliche Verwendung der untransformierten Gleichung im GMM-SYS hat darüber hinaus den Vorteil, dass auch zeitkonstante Faktoren berücksichtigt werden können. Dies ist im FE-Modell und im GMM-OR nicht möglich, da hier ausschließlich transformierte Daten verwendet werden.

Die Validität der verwendeten Instrumente im GMM-Modell wird durch den Hansen $J$ Test bzw. den Differenz-in Hansen Test überprüft (siehe Kapitel 7.2.2 Modellselektion). Der Hansen Test ist in der Situation mit vielen Instrumenten nicht effizient (ANDERSON und Sørensen, 1996). Aus diesem Grund werden in der empirischen Analyse nur Instrumente mit geringem zeitlichen Abstand zu den endogenen bzw. vordeterminierten Regressoren verwendet. ${ }^{46}$ Neben der Begrenzung der verwendeten Lags wird die Anzahl der Instrumente durch eine kollabierte Instrumentenmatrix reduziert (ROODMAN, 2008).

Für das GMM-OR und GMM-SYS wird ein zweistufiger Schätzansatz verwendet, da dieser robust gegenüber Heteroskedastizität und Korrelation innerhalb der und zwischen den Querschnittseinheiten ist (RoOdman, 2006: 8). ${ }^{47}$ Die Standardfehler der zweistufigen Schätzung sind nach unten verzerrt und werden entsprechend des Verfahrens nach WINDMEIJER (2000) korrigiert.

\subsubsection{Modellselektion}

Die Modellauswahl sollte auf Basis verschiedener Tests und Kriterien erfolgen. Grundsätzlich ist zu überprüfen, ob das Modell die getroffenen Annahmen erfüllt. Ist diese Voraussetzung gegeben, so können die Modelle hinsichtlich verschiedener Gütekriterien miteinander verglichen werden. Im Folgenden werden die wesentlichen Tests, die der Auswahl der diskutierten Panelmodelle zu Grunde liegen, diskutiert.

\section{Überprüfung der Modellannahmen}

Die zentralen Annahmen der diskutierten Panelmodelle sind iid-verteilte, nicht autokorrelierte Fehler, die Konstanz der Schätzer für alle Beobachtungen und die Exogenität von Regressoren bzw. Instrumenten.

46 Die in der empirischen Analyse verwendeten Instrumente sind in Tabelle 9.2 benannt.

47 BOND (2002: 9 f.) verweist darauf, dass der Effizienzgewinn durch den zweistufigen Schätzansatz relativ klein ist, auch im Fall des Vorliegens von Heteroskedastizität. 
Das Vorliegen von Autokorrelation wird durch den Arrellano-Bond-Test (ARRELLANO und BOND, 1991) überprüft. Der Test ist für lineare GMM- und OLS-Modelle anwendbar, robust gegenüber Heteroskedastizität und testet die Autokorrelation auf Basis der nicht transformierten Residuen (RoOdMAN, 2006).

Die Annahme konstanter Schätzer wird durch den Chow-Test, eine spezielle Anwendung des F-Tests bzw. des Log Likelihood Tests (LR-Test), verifiziert (Greene, 2003: 130). Hierfür werden ein vollständiges Modell mit allen Beobachtungen und ein restringiertes Modell, bestehend aus $n$ Teilmodellen, spezifiziert. Unterscheiden sich das vollständige Modell und die Summe der $n$ Teilmodelle nicht hinsichtlich ihres Erklärungsgehaltes, so wird vereinfachend von der Gleichheit der geschätzten Koeffizienten ausgegangen.

Die Verfügbarkeit valider Instrumente vorausgesetzt, können exogene Regressoren mit Hilfe des Hausman Tests identifiziert werden (GREenE, 2003: 301). Der Hausman Test überprüft die Nullhypothese der Gleichheit der geschätzten Koeffizienten zweier Modelle (z. B. OLS und 2SLS). Im Fall eines endogenen Regressors ist der OLS-Schätzer inkonsistent und weicht vom 2SLS-Schätzer ab. Sind alle Regressoren beider Modelle exogen, so sind beide Schätzer identisch. Durch den Hausman Test kann auch die Wahl des FEModells (gegenüber dem effizienteren Random-Effekt Modell) abgesichert werden. Im FE-Modell darf der unbeobachtete, betriebsspezifische Effekt $\left(\eta_{i}\right)$ willkürlich mit den Regressoren $\left(w_{i t}, x_{i t}\right)$ korrelieren, während $\eta_{i}$ im Random-Effekt-Modell unabhängig von den übrigen Regressoren verteilt sein muss. Unterscheiden sich die Koeffizienten beider Modelle signifikant, so korreliert $\eta_{i}$ mit Elementen in $w_{i t}$ und $x_{i t}$. Die zentrale Annahme des Random-Effekt Modells ist verletzt; es sollte ein FE-Modell verwendet werden.

Die gemeinsamen Validität (d. h. Exogenität) der verwendeten Instrumente (Momentbedingungen) wird in den GMM-Modellen durch den Hansen $J$ Test bzw. den Sargan Test überprüft (HANSEN, 1982; SARGAN, 1958). Voraussetzung hierfür ist, dass das Modell überidentifiziert ist, $\mathrm{d}$. h. mehr Instrumente als $\mathrm{zu}$ instrumentierende Variablen verwendet werden. Im Gegensatz zum Hansen $J$ Test ist der Sargan Test nicht robust gegenüber Heteroskedastizität und Autokorrelation (RoOdMAN, 2006) und wird daher nicht verwendet. Mit Hilfe der Differenz-in-Hansen Statistik kann die Exogenität einzelner Instrumente überprüft werden. Die Differenz-in-Hansen Statistik basiert auf der Differenz des Hansen $J$ Tests zweier Modelle: einem Modell mit allen Instrumenten und einem Modell ohne die auf Exogenität zu testenden Instrumente. Überprüft wird die Nullhypothese, dass die Instrumentensets beider Modelle exogen und damit valide sind (BAUM et al., 2003). 


\section{Vergleich von Modellen}

Verschachtelte ${ }^{48}$ Modelle können hinsichtlich ihres Erklärungsgehaltes mit Hilfe des LRTests (bzw. F-Tests) miteinander verglichen werden. Wie im Chow-Test werden ein vollständiges und ein restringiertes Modell spezifiziert. Mögliche Restriktionen können sein (LUDWIG-MAYERHOFER, 2002):

- Alle Parameter werden auf Null gesetzt (Nullmodell). Es wird getestet, ob das Nullmodell mehr erklärt als das spezifizierte Modell $\left(H_{0}: \beta_{i}=0\right)$.

- Ein oder mehrere Parameter werden auf Null gesetzt. Es wird getestet, ob der/die auf Null gesetzte(n) Parameter einen signifikanten Einfluss auf das Ergebnis hat/haben $\left(H_{0}: \beta_{1}=0, \beta_{2}=0\right)$.

- Zwei Parameter sollen identisch sein oder einen definierten, von Null verschiedenen Wert annehmen. Es wird getestet, ob die beiden Parameter einen gleich starken und gleich gerichteten Einfluss auf das Ergebnis haben oder ob der Einfluss über bzw. unterhalb eines definierten Wertes liegt $\left(H_{0}: \beta_{1}=\beta_{2}=1\right)$.

Der Erklärungsgehalt von nicht verschachtelten Modellen kann auf Basis des Bestimmtheitsmaßes $\left(\mathrm{R}^{2}\right)$, des Bayesianischen Informationskriteriums (BIC) oder des AkaikeInformationskriteriums (AIC) verglichen werden. Das $\mathrm{R}^{2}$ spiegelt den Anteil der durch das Modell erklärten Varianz wider und nimmt Werte zwischen 0 und 1 an. Das $\mathrm{R}^{2}$ favorisiert Modelle mit möglichst vielen Variablen. Das korrigierte $\mathrm{R}^{2}$ berücksichtigt dagegen auch die Modellkomplexität (Anzahl der Regressoren) in Form eines Strafterms. BIC und AIC basieren auf dem Log Likelihood zuzüglich eines Strafterms, der die Komplexität des Modells (Anzahl Modellparameter, Anzahl Beobachtungen) widerspiegelt. BIC sollte bevorzugt werden, wenn mehr Gewicht auf eine minimalistische Modellspezifikation gelegt wird. AIC bewertet komplexe Modelle mit einer hohen Anzahl an Modellparametern vergleichsweise günstiger als BIC. Je kleiner die Werte des BIC/AIC, desto vorzüglicher ist das Modell (CAMERON und TRIVEDI, 2005: 287ff).

Die Auswahl des zu bevorzugenden Modells ist in der Regel ein iterativer Prozess. Die im Modell enthaltenen Regressoren sollten primär auf Basis theoretischer Überlegungen ausgewählt werden und nicht auf Basis der Signifikanz der geschätzten Parameter. Letzteres führt zu einer Überschätzung der Modellgüte und möglicherweise zu zufälligen und unplausiblen Ergebnissen. In der Praxis ist das Auffinden valider Instrumente häufig schwierig, so dass eine rein theoriebasierte Instrumentenwahl immer durch entsprechende Tests abzusichern ist. Eine gute Praxis ist es, für die Suche nach der richtigen Modellspezifika-

48

Zwei Modelle sind verschachtelt, wenn beide Modelle die gleichen Regressoren umfassen und ein Modell mindestens einen zusätzlichen Regressor. Die Anzahl der Beobachtungen muss in beiden Modellen gleich groß sein. 
tion eine Teilstichprobe zu verwenden und die finale Modellschätzung auf Basis der verbleibenden Teilstichprobe vorzunehmen (CAMERON und TRIVEDI, 2005: 286).

\subsubsection{Analyse heterogener Programmeffekte}

Für die Analyse heterogener Programmeffekte werden die in Kapitel 7.2.1 vorgestellten Standardmodelle um Interaktionsterme erweitert. Der Einfluss der Höhe von Programmzahlungen auf den betrieblichen Faktoreinsatz wird mit Hilfe eines Selektionsmodells nach HECKMAN (1976) ermittelt.

\section{Panelregression mit Interaktionstermen}

Die Verwendung von Interaktionstermen zur Analyse heterogener Programmeffekte geht von der Annahme aus, dass das Niveau des Effektes der Programmteilnahme, $d_{i t}$, auf den betrieblichen Faktoreinsatz, $y_{i t}$, in Abhängigkeit von einer weiteren Variablen (im Folgenden Moderatorvariable), $g_{i t}$, variiert (BAUER und CURRAN, 2005). Die in den vorgestellten Standardmodellen geltende Annahme additiver Separabilität wird, bezogen auf den Effekt der Programmteilnahme, aufgehoben. Dargestellt am Beispiel des POLS aus Gleichung 7.10 hat das entsprechende Modell mit Interaktionstermen die Form

$$
y_{i t}=\gamma w_{i t}+\beta x_{i t}+\delta_{1} d_{i t}+\delta_{2} d_{i t} g_{i t}+\zeta g_{i t}+u_{i t}, \quad i=1, \ldots, N, t=1, \ldots, T
$$

wobei das interessierende Ergebnis, $y_{i t}$, die Vektoren der Regressoren, $w_{i t}, x_{i t}, d_{i t}, g_{i t}$ die zu schätzenden Koeffizienten, $\gamma, \beta, \delta_{1}, \zeta$ sowie der Fehlerterm $u_{i t}$ wie im POLS definiert sind. Der Interaktionsterm zwischen der Programmteilnahme und der stetig skalierten Moderatorvariable, $d_{i t} g_{i t}$, ist ein $1 \times 1$ Vektor. Der Effekt der Programmteilnahme auf das interessierende Ergebnis, in Abhängigkeit von der Moderatorvariablen, entspricht dem zu schätzenden Koeffizienten $\delta_{2}$.

An der Modellspezifikation in (7.16) wird deutlich, dass der Einfluss der Programmteilnahme auf das interessierende Ergebnis von der Ausprägung der Moderatorvariablen, z. B. der Betriebsgröße, abhängt. Ist die Moderatorvariable stetig skaliert, so ist es üblich, den Einfluss des Interaktionsterms für bestimmte Parameter (z. B. Mittelwerte) zu überprüfen. Eine weitere Möglichkeit besteht in der Bestimmung von Bereichen, in denen $d_{i t} g_{i t}$ einen signifikanten Effekt auf das interessierende Ergebnis hat (BAUER und Curran, 2005).

Abweichend von dem beschriebenen Ansatz, werden zur Analyse heterogener Programmeffekte ausschließlich diskret skalierte Moderatorvariablen verwendet. Die Moderatorvariable in Modell (7.16) entspricht in diesem Fall $n$ Dummyvariablen für die Teilnehmer der Klassen $1, \ldots, n$. Stetige Merkmale (z. B. Betriebsgrößenklassen) werden in eine diskrete 
Skalierung überführt. Hierdurch gehen zwar Informationen verloren; die Überprüfung der Unterschiede zwischen einzelnen Gruppen (Teilnehmer vs. Nichtteilnehmer, Teilnehmer Gruppe A vs. Teilnehmer Gruppe B) wird aber wesentlich erleichtert.

\section{Selektionsmodell nach Heckman}

Die Programmzahlungen betragen für Nichtteilnehmer Null Euro und nehmen für Teilnehmer einen Wert größer Null an. Die Berücksichtigung der Programmzahlungen in einem linearen Regressionsmodell würde, aufgrund der linksseitigen Stauchung der Variablen, vermutlich zu verzerrten Schätzergebnissen führen. Das von HECKMAN (1976) vorgeschlagene Selektionsmodell bietet für diesen speziellen Fall eine Lösung und ist ein Standardverfahren für die Programmevaluation (WoOldRIDGE, 2002: 563-466). Das Heckman-Modell kann ohne größere Modifikationen in Verbindung mit gepoolten, untransformierten Paneldaten (POLS) angewendet werden.

Die Anwendung von Selektionsmodellen in Verbindung mit FE-Modellen und dynamischen GMM-Modellen ist grundsätzlich möglich (KYRIAZIDOU, 2001; SEMYKINA und WOOLDRIDGE, 2005; WoOLDRIDGE, 1995), aber an mehrere Voraussetzungen gebunden: i) Der Selektionsmechanismus darf nicht durch unbeobachtete Effekte beeinflusst sein (Wooldridge, 1995). Diese Hypothese wurde überprüft und abgelehnt. ii) Die selektierte Unterstichprobe darf nur vollständige (balancierte) Panels enthalten. Bezogen auf die AUM- und AZ-Teilnahme bedeutet dies, dass der Effekt der Programmteilnahme nur für Betriebe, die immer an der Maßnahme teilgenommen haben, geschätzt werden kann. In der Panelregression variiert jedoch der Status Programmteilnahme zwischen einzelnen Untersuchungsjahren und ist nicht auf ,immer“ oder „nie“ beschränkt. Aus diesem Grund wird das Selektionsmodell nur in Verbindung mit dem POLS verwendet.

Das Heckman-Modell ist ein zweistufiger Schätzansatz: Im ersten Schritt wird die Entscheidung der Betriebe zur Programmteilnahme modelliert (Selektionsgleichung). Im zweiten Schritt wird der Effekt der Programmteilnahme auf das interessierende Ergebnis, unter Berücksichtigung eines Korrekturterms, geschätzt (Ergebnisgleichung). Das verwendete Heckman-Modell ist formal beschrieben als

$$
\begin{array}{ll}
d_{i t}^{*}=\psi g_{i t}+\varepsilon_{i t} & d_{i t}=\left\{\begin{array}{l}
1, \text { wenn } d_{i t}^{*}>0 \\
0, \text { ansonsten },
\end{array}\right. \\
y_{i t}=\gamma w_{i t}+\beta x_{i t}+\delta d_{i t}+u_{i t} & d_{i t}=1, i=1, \ldots, N, t=1, \ldots, T,
\end{array}
$$

wobei $d_{i t}^{*}$ den Indikator der Programmteilnahme repräsentiert, der für Teilnehmer den Wert 1 und ansonsten den Wert Null annimmt. $g_{i t}$ ist ein $1 \times K$ Vektor beobachteter Determinanten der Programmteilnahme, $\psi$ ein $k \times 1$ Vektor der zu schätzenden Koeffizienten und $\varepsilon_{i t}$ der zufällige Fehler. $g_{i t}$ kann exogene Regressoren und Lags endogener 
Regressoren der Ergebnisgleichung umfassen $\left(w_{i, t-1}, x_{i t}\right)$. Als Ergebnisgleichung wird das Modell POLS aus Gleichung (7.10) verwendet.

Zur Kontrolle des Selektionseffektes wird die Korrelation zwischen dem Fehlerterm der Selektionsgleichung, $\varepsilon_{i t}$, und dem Fehlerterm der Ergebnisgleichung, $u_{i t}$, genutzt. Eine strenge Annahme des Selektionsmodells ist die bivariate Normalverteilung der beiden Fehler $\varepsilon_{i t}$ und $u_{i t}$. Obwohl diese Annahme vermutlich selten erfüllt ist, werden Selektionsmodelle sehr häufig verwendet (GREENE, 2003: 789).

Für die empirische Analyse wird ein zweistufiger OLS-Schätzer verwendet, da der alternative Maximum-Likelihood-Schätzer in großen Datensätzen einen hohen Rechenaufwand erfordert. Die Entscheidung zur Programmteilnahme (Selektionsgleichung) wird durch ein binäres Probit-Modell geschätzt

$$
\operatorname{Pr}\left(d_{i t}=1 \mid g_{i t}\right)=\Phi\left(\psi g_{i t}\right)
$$

Aus der in Gleichung (7.18) geschätzten Wahrscheinlichkeit der Programmteilnahme wird der Korrekturterm $\lambda_{i t}$ (Lambda oder das Inverse von Mills Ratio) entsprechend

$$
\lambda_{i t}= \begin{cases}\phi\left(\hat{\psi} g_{i t}\right) / \Phi\left(\hat{\psi} g_{i t}\right), & d_{i t}=1 \\ -\phi\left(\hat{\psi} g_{i t}\right) /\left(1-\Phi\left(\hat{\psi} g_{i t}\right)\right), & d_{i t}=0\end{cases}
$$

berechnet, wobei $\phi$ und $\Phi$ die Wahrscheinlichkeitsdichte und die kumulative Verteilungsfunktion der Standardnormalverteilung sind. Die Ergebnisgleichung wird für die Unterstichprobe der Betriebe mit Programmteilnahme, unter Berücksichtigung des Korrekturterms, $\lambda_{i t}$, geschätzt. Der zweistufige Schätzansatz erfordert eine Anpassung der Standardfehler in der Ergebnisgleichung.

Alle Regressionsschätzungen werden mit Stata (2007) durchgeführt. Neben den in Stata enthaltenen Standardprogrammen wurden verwendet: xtabond2 für GMM-OR und GMMSYS und abar zum Test auf Autokorrelation in Paneldaten (RoOdMAn, 2006). 


\subsection{Zusammenfassung}

Um die Effekte der Teilnahme an Agrarumweltmaßnahmen und an der Ausgleichszulage auf die betriebliche Faktorausstattung zu schätzen, werden ein semi-parametrisches Matching-Verfahren und verschiedene parametrische Panelregressionsmodelle verwendet.

Das verwendete semi-parametrische Verfahren ist ein Kontrollgruppenvergleich in Verbindung mit dem Propensity Score Matching. Der Effekt der Programmteilnahme wird als Differenz zwischen der Ergebnisänderung (2000-2005) von vergleichbaren Teilnehmerund nicht teilnehmenden Kontrollbetrieben geschätzt (Differenz-in-Differenz-Schätzung, DiD). Vergleichbare Teilnehmer- und Kontrollbetriebe werden auf Basis beobachteter Merkmale identifiziert (selection on observables). Durch die DiD-Schätzung kann der Einfluss zeitkonstanter, unbeobachteter, betriebsspezifischer Faktoren auf das interessierende Ergebnis kontrolliert werden. Zur Analyse heterogener Programmeffekte in Abhängigkeit von betrieblichen und regionalen Merkmalen werden nicht-parametrische Regressionsmodelle und das stratifizierte Matching verwendet.

Für die parametrische empirische Analyse werden vier Regressionsmodelle als Standardverfahren eingesetzt (gepooltes OLS-Modell, Fixed-Effekt-Modell, GMM-Modell mit orthogonalen Abweichungen, System GMM-Modell). Entsprechend den Modellannahmen, sollte die Verzerrtheit der Schätzer vom gepoolten OLS-Modell hin zum System GMMModell abnehmen. Mit Ausnahme des gepoolten OLS-Modells erlauben alle Modelle die Kontrolle des Selektionseffektes aufgrund unbeobachteter Faktoren (selection on unobservables). Die beiden GMM-Modelle ermöglichen darüber hinaus die aufgrund der empirischen Fragestellung wünschenswerte dynamische Modellspezifikation und die Berücksichtigung endogener Regressoren. Zur Analyse heterogener Effekte der Programmteilnahme werden die Standardmodelle um Interaktionsterme ergänzt bzw. das gepoolte OLS-Modell zu einem Selektionsmodell nach Heckman modifiziert. 


\section{Empirische Ergebnisse der Matching-Analyse}

In diesem Kapitel sind die Ergebnisse des Propensity Score Matching dargestellt. Ermittelt wurden die Effekte der Teilnahme an Agrarumweltmaßnahmen (AUM) und an der Ausgleichszulage (AZ) auf den betrieblichen Faktoreinsatz, den Umsatz und die außerbetriebliche Erwerbstätigkeit des Betriebsleiterpaares im Untersuchungszeitraum 2000 bis 2005.

Die empirische Modellspezifikation sowie die deskriptive Statistik der verwendeten Daten sind in Kapitel 8.1 dargestellt. Im Kapitel 8.2 wird die Anwendung des Propensity Score Matching demonstriert. Der mittlere Effekt der Teilnahme an Agrarumweltmaßnahmen und an Ausgleichszulage ist in Kapitel 8.3 beschrieben. Aufgrund des flächenmäßig variierenden Teilnahmeumfangs von Betrieben an AUM und der AZ, der unterschiedlichen Ausgestaltung der Programme zwischen den Bundesländern und den verschiedenen Anpassungsstrategien von Betrieben an die Auflagen der Programme ist zu vermuten, dass der Effekt der Programmteilnahme in Abhängigkeit von der Höhe der Programmzahlung, der Region, der Betriebsform, der Betriebsgröße und der Teilnahmewahrscheinlichkeit variiert. Inwieweit die Programmeffekte in Abhängigkeit von diesen Faktoren variieren, wird in Kapitel 8.4 gezeigt. Die Ergebnisse der Matching-Analyse sind in Kapitel $8.5 \mathrm{zu}-$ sammengefasst.

\subsection{Empirische Spezifikation des verwendeten Matching-Modells}

Das verwendete Matching-Modell mit DiD-Schätzer ist in allgemeiner Form in Kapitel 7.1 beschrieben. Die deskriptive Statistik der für die Matching-Analyse verwendeten Daten ist der Tabelle A4 im Anhang zu entnehmen.

Der Effekt der AUM-Teilnahme auf die Teilnehmer $\left(A T T_{A U M}\right)$ entspricht der Differenz der Ergebnisänderung (2000 bis 2005) zwischen vergleichbaren Teilnehmern (AUM=1) und Kontrollbetrieben $(\mathrm{AUM}=0)$ vor und nach der Programmdurchführung. Vergleichbare Teilnehmer- und Kontrollbetriebe werden auf Basis beobachteter Merkmale bzw. des hieraus geschätzten Propensity Score, im Referenzjahr 2000, gebildet $\left(p\left(X_{2000}\right)\right)$.

$$
A T T_{A U M}=E\left[Y_{2005}^{1}-Y_{2000}^{0} \mid A U M=1, p\left(X_{2000}\right)\right]-\left[Y_{2005}^{0}-Y_{2000}^{0} \mid A U M=0, p\left(X_{2000}\right)\right] .
$$

AUM-Teilnehmer haben im Referenzjahr 2000 keine und zwischen 2001 bis 2005 kontinuierlich positive Programmzahlungen (Agrarumweltprämien) erhalten. Nichtteilnehmer haben in keinem Jahr zwischen 2000 und 2005 positive Programmzahlungen erhalten. 
Für die Ausgleichszulage ist der Zeitpunkt vor dem Programmstart nicht in den Buchführungsdaten enthalten, da die Mehrheit der AZ-Betriebe die Ausgleichszulage seit über 20 Jahren erhält (vgl. Kapitel 2.3). Folglich kann das Ergebnis eines AZ-Teilnehmers vor Erhalt der Ausgleichszulage nicht beobachtet werden. Aus diesem Grund muss für die Ausgleichszulage eine von Gleichung (8.1) abweichende Modellspezifikation gewählt werden, die die kontinuierliche Inanspruchnahme der Ausgleichszulage berücksichtigt.

$$
A T T_{A Z}=E\left[Y_{2005}^{1}-Y_{2000}^{1} \mid A Z=1, p\left(X_{2000}\right)\right]-\left[Y_{2005}^{0}-Y_{2000}^{0} \mid A Z=0, p\left(X_{2000}\right)\right] .
$$

AZ-Teilnehmer $(A Z=1)$ haben sowohl in 2000 als auch in 2005 eine Ausgleichszulage erhalten; Nichtteilnehmer $(A Z=0)$ haben in keinem Jahr zwischen 2000 und 2005 Zahlungen im Rahmen der Ausgleichszulage erhalten. Statt des Ergebnisses vor dem Programmstart $\left(Y_{2000}^{0}\right)$ wird in Gleichung (8.1) das Ergebnis unter andauernder Programmteilnahme $\left(Y_{2000}^{1}\right)$ verwendet.

Die kombinierte Teilnahme an Agrarumweltmaßnahmen und der Ausgleichszulage kann aufgrund der zu geringen Anzahl von Stichprobenbetrieben, die an beiden Programmen teilnehmen, nicht analysiert werden.

Der durchschnittliche Effekt der Programmteilnahme wird durch den Vergleich der Ergebnisänderungen der Teilnehmer an AUM (AZ) $\Delta y_{i}^{1}=y_{i, 2005}^{1}-y_{i, 2000}^{0}\left(\Delta y_{i}^{1}=y_{i, 2005}^{1}-y_{i, 2000}^{1}\right)$ und der Kontrollbetriebe $\Delta y_{j}^{0}=y_{j, 2005}^{0}-y_{j, 2000}^{0}$ ermittelt. Der mittlere kausale Effekt der Programmteilnahme auf die Teilnehmer (ATT) berechnet sich als die mittlere Differenz der Ergebnisänderung beider Gruppen

$$
A T T_{A U M, A Z}=\frac{1}{N}\left(\sum_{i=1}^{N} \Delta y_{i}^{1}-\sum_{j=1}^{N} \Delta y_{j}^{0}\right)
$$

wobei $N$ die Anzahl der Teilnehmer- und Kontrollbetriebe darstellt.

\subsection{Anwendung des Propensity Score Matching}

Die Auswahl vergleichbarer Betriebe erfolgt auf Basis des Propensity Score. Der Propensity Score wird, jeweils getrennt für Agrarumweltmaßnahmen und die Ausgleichszulage, in einem Logit-Modell geschätzt.

Tabelle 8.1 stellt die Parameterschätzwerte der Logit-Modelle zur Erklärung der Teilnahme an Agrarumweltmaßnahmen und an der Ausgleichszulage für die gesamtdeutsche Stichprobe dar. 
Tabelle 8.1: Parameterschätzwerte der Logit-Modelle zur Erklärung der AUM- und AZ-Teilnahme, Deutschland gesamt

\begin{tabular}{|c|c|c|c|c|c|c|}
\hline \multirow[t]{2}{*}{ Merkmal (2000) } & \multirow[t]{2}{*}{ Einheit } & \multicolumn{2}{|c|}{ Agrarumweltprogramme } & \multicolumn{3}{|c|}{ Ausgleichszulage } \\
\hline & & Schätzer & Wald $\mathrm{Chi}^{2}$ & Schätzer & Wald $\mathrm{Chi}^{2}$ & \\
\hline \multicolumn{7}{|l|}{ Betriebliche Merkmale } \\
\hline $\ln L F$ & ha & 1,815 & $12,073 * * *$ & & & \\
\hline $\ln L F$ (quadriert) & ha & $-0,242$ & $25,479 * * *$ & & & \\
\hline ln Grünland & ha & 0,340 & $102,417 * * *$ & & & \\
\hline Anteil Grünland & $\%$ & & & 0,549 & 184,473 & $* * *$ \\
\hline In Anteil Pachtland & $\%$ & 0,057 & $6,458 * *$ & 0,054 & 3,962 & $* *$ \\
\hline ln AK Betrieb & AKE & 0,058 & 0,711 & $-0,252$ & 8,497 & $* * *$ \\
\hline $\ln$ Außerbetriebliche $A K$ & Stunden/Jahr & 0,015 & 2,030 & & & \\
\hline In Außerbetriebliche $A K B L$ & Stunden/Jahr & 0,065 & 0,463 & & & \\
\hline ln Rinderbesatz & GV & 0,031 & 0,697 & 0,031 & 0,490 & \\
\hline In Rinderbesatzdichte & GV & $-0,508$ & $50,786 * * *$ & $-0,350$ & 14,284 & $* * *$ \\
\hline ln Kapital (je ha) & TEuro & $-0,142$ & $13,757 * * *$ & & & \\
\hline In Ausgaben Dünger (je ha) & TEuro & $-0,187$ & $21,941 * * *$ & $-0,160$ & 12,441 & $* * *$ \\
\hline In Ausgaben PSM (je ha) & TEuro & 0,135 & $29,560 * * *$ & $-0,017$ & 0,345 & \\
\hline In Viehbestand (je 100 ha) & VE & 0,021 & 0,889 & & & \\
\hline ln Tierprämien (je ha) & TEuro & $-0,032$ & $4,540 * *$ & & & \\
\hline In Flächenprämien (je ha) & TEuro & $-0,218$ & $50,569 * * *$ & 0,018 & 0,252 & \\
\hline AZ-Zahlungen (je ha) & TEuro & 2,663 & $3,082 *$ & & & \\
\hline AUM-Teilnahme & $0=$ nein, $1=\mathrm{ja}$ & & & $-0,047$ & 0,054 & \\
\hline AZ-Teilnahme & $0=$ nein, $1=j a$ & 0,358 & $14,031 * * *$ & & & \\
\hline Ord. Ergebnis & TEuro & 0,004 & $11,165 * * *$ & 0,007 & 18,549 & $* * *$ \\
\hline Ord. Ergebnis (je ha) & TEuro & & & $-0,266$ & 10,334 & $* * *$ \\
\hline Ord. Ergebnis $+P A / A K$ & TEuro & $-0,001$ & 1,023 & & & \\
\hline ln Umsatz & TEuro & 0,711 & $4,285 * *$ & & & \\
\hline ln Umsatz (je ha) & TEuro & $-1,025$ & $8,558 * * *$ & $-0,840$ & 65,772 & $* * *$ \\
\hline Ldw. Bildung $($ Uni/FH) & $0=$ nein, $1=$ ja & 0,251 & $14,608 * * *$ & 0,036 & 0,313 & \\
\hline Ldw. Bildung (Techniker) & $0=$ nein, $1=\mathrm{ja}$ & 0,341 & $3,437 *$ & & & \\
\hline Ldw. Bildung (Jahre) & Anzahl Jahre & 0,035 & 2,096 & & & \\
\hline Nldw. Bildung (Uni/FH) & $0=$ nein, $1=\mathrm{ja}$ & $-0,073$ & 0,649 & $-0,351$ & 10,669 & $* * *$ \\
\hline Ackerbau & $0=$ nein, $1=\mathrm{ja}$ & $-0,153$ & 2,424 & $-0,122$ & 0,948 & \\
\hline Futterbau & $0=$ nein, $1=\mathrm{ja}$ & $-0,362$ & $18,921 * * *$ & $-0,024$ & 0,068 & \\
\hline Veredlung & $0=$ nein, $1=\mathrm{ja}$ & $-0,231$ & $3,568 *$ & & & \\
\hline Gemischt & $0=$ nein, $1=\mathrm{ja}$ & $-0,212$ & $3,425 *$ & 0,178 & 1,812 & \\
\hline$L V Z$ & Index & $-0,010$ & $17,393 * * *$ & $-0,201$ & $1.923,219$ & $* * *$ \\
\hline Höhe & Höhe über N. N. & 0,003 & $107,207 * * *$ & 0,004 & 165,561 & $* * *$ \\
\hline \multicolumn{7}{|l|}{ Regionale Merkmale } \\
\hline Quote & $\%$ & 0,066 & $21,508 * * *$ & & & \\
\hline BWS Landwirtschaft & 1.000 Euro & 0,001 & $4,756 * *$ & 0,006 & 74,707 & $* * *$ \\
\hline $\ln B I P($ pro Kopf) & 1.000 Euro & 0,244 & $19,883 * * *$ & 0,286 & 25,397 & $* * *$ \\
\hline In Einwohnerdichte & EW/qm & 0,267 & 2,428 & $-2,098$ & 12,419 & $* * *$ \\
\hline In Einwohner-Arbeitsplatz-Dichte & EW/Erw. & 0,055 & 0,087 & 1,219 & 4,592 & $* *$ \\
\hline Erreichbarkeit & Minuten & 0,008 & $9,648 * * *$ & $-0,008$ & 6,001 & $* *$ \\
\hline Anteil ländliche Bevölkerung & Index & $-0,014$ & $33,439 * * *$ & 0,005 & 2,655 & \\
\hline $\ln$ Pacht & Euro/ha & & & $-0,005$ & 109,144 & $* * *$ \\
\hline Anteil Futterbau & $\%$ & $-0,023$ & $111,510 * * *$ & $-0,022$ & 44,881 & $* * *$ \\
\hline Anteil Ackerbau & $\%$ & $-0,005$ & $4,843 * *$ & $-0,034$ & 108,266 & $* * *$ \\
\hline Anteil Veredlung & $\%$ & 0,003 & 0,160 & $-0,170$ & 416,459 & $* * *$ \\
\hline Anteil Gemischt & $\%$ & 0,097 & $51,868 * * *$ & 0,346 & 363,588 & $* * *$ \\
\hline Dummyvariablen Region & $0=$ nein, $1=\mathrm{ja}$ & & & & & \\
\hline Region Nord & $0=$ nein, $1=\mathrm{ja}$ & $-0,034$ & 0,027 & $-8,078$ & 527,258 & $* * *$ \\
\hline Region West & $0=$ nein, $1=\mathrm{ja}$ & $-0,840$ & $14,323 * * *$ & 0,297 & 8,291 & $* * *$ \\
\hline Region Süd & $0=$ nein, $1=\mathrm{ja}$ & 3,830 & $201,156 * * *$ & & & \\
\hline Anzahl Beobachtungen & $\mathrm{N}$ & & 19.337 & & 23.120 & \\
\hline LR Chi-Quadrat & & & $14.482,79 * * *$ & & $20.909,29$ & $* * *$ \\
\hline Pseudo $\mathrm{R}^{2}$ & & & 0,70 & & 0,83 & \\
\hline$\%$ richtiger Vorhersagen & & & 87,49 & & 92,66 & \\
\hline Kontrollbetriebe & & & 90,77 & & 95,10 & \\
\hline Teilnehmer & & & 84,37 & & 87,55 & \\
\hline
\end{tabular}

Signifikanzniveau: $* * * \mathrm{p}<0,01, * * \mathrm{p}<0,05, *<0,1$.

Quelle: eigene Berechnung, Datenquellen vgl. Tabellen A2 und A3. 
Der LR-Test zeigt, dass die Signifikanz der geschätzten Modelle $1 \%$ oder besser ist. Die Vorhersagegüte der Modelle ist mit 70 \% (AUM) bzw. $83 \%$ (AZ) Varianzaufklärung sehr gut. Durch das AUM-Modell (AZ-Modell) werden $86 \%$ (93\%) aller Beobachtungen richtig klassifiziert. Für diesen Anteil der Betriebe wird die Teilnahme an AUM (AZ) richtig vorhergesagt. Aus diesem Ergebnis ist zu folgern, dass die Mehrzahl der Determinanten der Programmteilnahme in den Modellen berücksichtigt wurde.

Die erklärenden Variablen der Logit-Modelle beschreiben den Selektionsmechanismus zur Teilnahme an AUM und AZ. So hat z. B. die Landwirtschaftliche Vergleichszahl (LVZ) einen negativen und die Höhenlage des Betriebes einen positiven Einfluss auf die Wahrscheinlichkeit der AUM- bzw. AZ-Teilnahme: Je geringer die LVZ bzw. je höher die Höhenlage (Höhe) eines Betriebes, desto größer ist die Wahrscheinlichkeit für die Programmteilnahme. Auf diesen Zusammenhang wurde bereits in Kapitel 2 verwiesen. Im Vergleich zur AZ ist die Teilnahme an AUM stärker durch einzelbetriebliche Merkmale determiniert. Der Grund hierfür liegt in den einzelbetrieblich sehr unterschiedlichen Anpassungskosten an die Bewirtschaftungsauflagen von AUM. Die Teilnahme an der AZ wird im Wesentlichen durch die Gebietskulisse determiniert, deren Abgrenzung auf Basis der Standortgüte (LVZ, Höhe über N. N.), aber auch auf Basis sozioökonomischer Kriterien erfolgte.

Neben den Determinanten der Programmteilnahme umfassen die erklärenden Variablen der Logit-Modelle ausgewählte Ergebnisvariablen im Referenzjahr 2000 (pre-treatment outcomes), regionale Merkmale (z. B. Arbeitslosenquote) und Regionsdummies (Nord, West, Süd und Ost). Die Verwendung von pre-treatment outcomes stellt sicher, dass die zu vergleichenden Teilnehmer- und Kontrollbetriebe im Referenzjahr 2000 eine ähnliche Ausgangssituation hinsichtlich ihrer betrieblichen Faktorausstattung aufweisen. Durch die Berücksichtigung regionaler Merkmale werden Paare aus Teilnehmern und vergleichbaren Kontrollbetrieben innerhalb einer Region gebildet. Beides, die Verwendung von pre-treatment outcomes sowie das lokale Matching sind Voraussetzungen zur Reduzierung des Selektionseffektes (COOK, SHADISH und Wong, 2007).

Für jeden Betrieb wird aus den geschätzten Koeffizienten der Propensity Score berechnet, auf dessen Grundlage das Matching der Teilnehmer und Kontrollbetriebe erfolgt.

Tabelle 8.2 zeigt für das Referenzjahr (2000) die Mittelwerte (Häufigkeiten) der Ergebnisvariablen von Teilnehmern und Kontrollbetrieben, vor dem Matching (Spalten (1) und (2) bzw. (6) und (7)) und nach dem Matching (Spalten (3) und (4) bzw. (8) und (9). Vor dem Matching unterscheiden sich die Teilnehmer an AUM (der AZ) von den Kontrollbetrieben in fast allen Merkmalen signifikant (Irrtumswahrscheinlichkeit $<5 \%$ ). Der Vergleich zwischen Spalte (1) und (2) zeigt, dass die AUM-Teilnehmer im Referenzjahr z. B. eine höhere $L F$, mehr Grünland und geringere Ausgaben für Düngemittel (je ha) aufweisen als die Nichtteilnehmer. Auch die AZ-Teilnehmer unterscheiden sich vor dem Matching in 
fast allen Merkmalen signifikant von den Nichtteilnehmerbetrieben (vgl. Spalte (6) und (7)).

Die Spalten (3) und (4) bzw. (8) und (9) zeigen die Mittelwerte (Häufigkeiten) der Ergebnisvariablen von Teilnehmern und Kontrollbetrieben nach dem Matching. Die Unterschiede zwischen den Mittelwerten der Teilnehmer- und Kontrollbetriebe wurden durch das Matching nahezu vollständig beseitigt und unterschreiten in keinem Fall das Signifikanzniveau von $5 \%$. Es kann gefolgert werden, dass der vor dem Matching bestehende Selektionseffekt aufgrund beobachteter Faktoren zwischen Teilnehmern und Kontrollbetrieben durch das Matching eliminiert wurde. Der Standardisierte Bias wurde für beide Programme deutlich reduziert (vgl. Tabelle 8.1).

Für das Matching stehen insgesamt 9.324 (AUM) bzw. 15.658 (AZ) Nichtteilnehmer und 10.013 (AUM) bzw. (7.462) Teilnehmerbetriebe zu Verfügung. Aus diesen Betrieben wurden je 1.460 (AUM) bzw. 1.693 (AZ) vergleichbare Teilnehmer- und Kontrollbetriebe selektiert. Etwa $85 \%$ (79\%) aller in der Stichprobe enthaltenen AUM-Teilnehmer (AZTeilnehmer) sind aus der Stichprobe ausgeschieden, da keine vergleichbaren Kontrollbetriebe aufzufinden waren.

In den Spalten (5) und (10) der Tabelle 8.2 sind die Merkmale der nicht selektierten AUM- und AZ-Teilnehmer dargestellt. Im Vergleich zu den selektierten Teilnehmern (Spalten 4 bzw. 9) wirtschaften die nicht selektierten Teilnehmerbetriebe extensiver (niedrigere Rinderbesatzdichte, weniger Ausgaben für Düngermittel je ha), sind größer und erhalten etwa doppelt so hohe Programmzahlungen je Hektar. ${ }^{49}$ So beträgt z. B. die durchschnittliche AUM-Zahlung je Hektar LF für die selektierten AUM-Teilnehmer 44 Euro und für die nicht selektierten AUM-Teilnehmer 84 Euro. Betriebe mit hohen Programmzahlungen je Hektar bewirtschaften vermutlich den überwiegenden Teil ihrer LF unter den Auflagen von AUM und AZ. Für diese Betriebe waren in der verwendeten Stichprobe zu wenig vergleichbare Betriebe ohne Programmteilnahme enthalten.

\footnotetext{
49 Die Programmzahlungen je Hektar LF entsprechen der Summe der Programmzahlungen je Betrieb geteilt
} durch die gesamte LF des Betriebes. Die tatsächliche Prämienhöhe je Hektar geförderter LF ist unbekannt. 
Tabelle 8.2: Vergleich von Mittelwerten (Häufigkeiten) für ausgewählte Variablen im Referenzjahr 2000, Greedy Matching, Deutschland gesamt

\begin{tabular}{|c|c|c|c|c|c|c|c|c|c|c|c|}
\hline \multirow[t]{3}{*}{ Variable (2000) } & \multirow[t]{3}{*}{ Einheit } & \multicolumn{5}{|c|}{ Agrarumweltmaßnahmen (AUM) } & \multicolumn{5}{|c|}{ Ausgleichszulage (AZ) } \\
\hline & & $\begin{array}{c}\text { Potenzielle } \\
\text { Kontroll- } \\
\text { betriebe }\end{array}$ & $\begin{array}{l}\text { Potenzielle } \\
\text { Teilnehmer }\end{array}$ & $\begin{array}{l}\text { Selektierte } \\
\text { Kontroll- } \\
\text { betriebe }\end{array}$ & $\begin{array}{l}\text { Selektierte } \\
\text { Teilnehmer }\end{array}$ & $\begin{array}{c}\text { Nicht } \\
\text { selektierte } \\
\text { Teilnehmer }\end{array}$ & $\begin{array}{c}\text { Potenzielle } \\
\text { Kontroll- } \\
\text { betriebe }\end{array}$ & $\begin{array}{l}\text { Potenzielle } \\
\text { Teilnehmer }\end{array}$ & $\begin{array}{l}\text { Selektierte } \\
\text { Kontroll- } \\
\text { betriebe }\end{array}$ & $\begin{array}{l}\text { Selektierte } \\
\text { Teilnehmer }\end{array}$ & $\begin{array}{c}\text { Nicht } \\
\text { selektierte } \\
\text { Teilnehmer }\end{array}$ \\
\hline & & (1) & (2) & (3) & (4) & (5) & (6) & (7) & (8) & (9) & \\
\hline $\ln L F$ & ha & 3,896 & 3,994 & 3,952 & 3,976 & 3,998 & 3,926 & 4,024 & 3,898 & 3,879 & 4,066 \\
\hline ln Grünland & ha & 1,792 & 2,303 & 1,987 & 2,051 & 2,346 & 1,646 & 2,774 & 2,245 & 2,190 & 2,946 \\
\hline ln Anteil Pachtland & $\%$ & 3,510 & 3,788 & 3,622 & 3,584 & 3,824 & 3,546 & 3,828 & 3,668 & 3,606 & 3,893 \\
\hline $\ln$ AK Betrieb & AKE & 0,298 & 0,441 & 0,335 & 0,350 & 0,456 & $\mathbf{0 , 3 4 0}$ & 0,436 & 0,419 & 0,410 & 0,444 \\
\hline $\ln$ AK Betrieb (je 100 ha) & AKE & 1,007 & 1,052 & 0,988 & 0,979 & 1,064 & 1,019 & 1,018 & 1,126 & 1,136 & 0,983 \\
\hline $\ln$ Außerbetriebliche AK & $\mathrm{h} / \mathrm{Jahr}$ & 1,243 & 1,120 & 1,215 & 1,151 & 1,107 & 1,269 & 1,067 & 1,135 & 1,088 & 1,061 \\
\hline ln Kapital (je ha) & TEuro & 2,364 & 2,413 & 2,360 & 2,345 & 2,419 & 2,408 & 2,365 & 2,479 & 2,474 & 2,333 \\
\hline ln Rinderbesatz & GV & 2,610 & 3,013 & 2,765 & 2,840 & 3,036 & 2,442 & 3,499 & 3,089 & 3,045 & 3,632 \\
\hline ln Rinderbesatzdichte & GV & 0,463 & 0,462 & 0,459 & 0,470 & 0,457 & 0,448 & 0,499 & 0,541 & 0,538 & 0,487 \\
\hline ln Ausgaben PSM (je ha) & TEuro & $-2,731$ & $-2,678$ & $-2,762$ & $-2,745$ & $-2,669$ & $-2,546$ & $-2,976$ & $-2,730$ & $-2,696$ & $-3,059$ \\
\hline ln Ausgaben Dünger (je ha) & TEuro & $-2,428$ & $-2,556$ & $-2,453$ & $-2,444$ & $-2,575$ & $-2,405$ & $-2,627$ & $-2,497$ & $-2,507$ & $-2,662$ \\
\hline ln Umsatz & TEuro & 4,776 & 4,676 & 4,734 & 4,749 & 4,658 & 4,787 & 4,646 & 4,686 & 4,668 & 4,639 \\
\hline ln Umsatz (je ha) & TEuro & 0,882 & 0,682 & 0,781 & 0,773 & 0,661 & 0,862 & 0,622 & 0,791 & 0,789 & 0,574 \\
\hline AUM-/AZ-Zahlungen $(j e ~ h a)^{*}$ & TEuro & $\mathbf{0}$ & 0,078 & 0 & 0,044 & 0,084 & $\mathbf{0}$ & 0,043 & 0 & 0,025 & 0,049 \\
\hline Region Nord & $0=$ nein, $1=\mathrm{ja}$ & 3.786 & 774 & 512 & 517 & 257 & 5.927 & $13 * *$ & 16 & 13 & 0 \\
\hline Region West & $0=$ nein, $1=\mathrm{ja}$ & 4.623 & 903 & 559 & 553 & 350 & 5.250 & 1.608 & 424 & 427 & 1.181 \\
\hline Region Süd & $0=$ nein, $1=\mathrm{ja}$ & 760 & 8.186 & 365 & 350 & 7.836 & 4.233 & 5.644 & 1.177 & 1.205 & 4.439 \\
\hline Region Ost & $0=$ nein, $1=\mathrm{ja}$ & 155 & 150 & 24 & 40 & 110 & 248 & 197 & 76 & 48 & 149 \\
\hline Anzahl Betriebe & & 9.324 & 10.013 & 1.460 & 1.460 & 8.553 & 15.658 & 7.462 & 1.693 & 1.693 & 5.769 \\
\hline
\end{tabular}

1. * Mittelwerte 2001 bis 2005 .

2. ** Die AZ wird in Niedersachsen nicht und in Schleswig-Holstein nur in einer eingeschränkten Gebietskulisse angeboten (vgl. Kapitel 2.3.2).

3. Fett gedruckte Werte kennzeichnen die statistische Signifikanz im T-Test (Chi-Quadrat-Test) für die Gleichheit der Mittelwerte (Häufigkeiten) der

Teilnehmer- und Kontrollbetriebe auf einem Niveau von $<5 \%$.

Quelle: eigene Berechnungen, Datenquellen vgl. Tabellen A2 und A3. 


\section{Überprüfung der Modellannahmen ${ }^{50}$}

Eine Überprüfung der Conditional Independence Assumption (CIA) ist praktisch nicht möglich (LECHNER, 2005). Der Matching-Ansatz basiert auf dem Prinzip selection on observables. Eine Verletzung der CIA ist möglich, wenn unbeobachtete Merkmale die Entscheidung zur Programmteilnahme beeinflussen. In der Logit-Analyse wurde die Entscheidung zur Programmteilnahme in 85 bis $90 \%$ der Fälle richtig vorhergesagt (vgl. Tabelle 8.1). Hieraus ist zu schließen, dass die Teilnahmeentscheidung der Betriebe weitgehend durch beobachtete Merkmale erklärt werden kann. Dies dürfte vor allem auf die hohe Anzahl an Beobachtungen (AUM: 19.337, AZ: 23.220) zurückzuführen sein.

Die CIA ist für die Ausgleichszulage verletzt, da für AZ-Teilnehmer keine Ergebnisse für die Situation vor Beginn des Programms vorliegen. Das im Referenzjahr 2000 gemessene Ergebnis ist nicht unabhängig vom Teilnahmestatus und damit nicht exogen (LECHNER, 2005). Hat z. B. der Erhalt der Ausgleichszulage vor 2000 das betriebliche Flächenwachstum positiv beeinflusst, so ist die Flächenausstattung von AZ-Teilnehmern im Referenzjahr 2000 höher als in der der Situation ohne das Programm. Eine praktikable Lösung zum Umgang mit endogenen Variablen im Matching ist nicht bekannt.

Die Common Support Condition ist in der empirischen Analyse nur teilweise erfüllt. Für viele Teilnehmer gibt es keinen vergleichbaren Kontrollbetrieb - entweder, weil die meisten vergleichbaren Betriebe ebenfalls am Programm teilnehmen, oder, weil mögliche Kontrollbetriebe in der Stichprobe kaum enthalten sind. Dies führt dazu, dass vorrangig Teilnehmer mit relativ geringen Programmzahlungen selektiert werden (vgl. Tabelle 8.2). Wenn ein systematischer Zusammenhang zwischen der Höhe der Programmzahlungen und dem Programmeffekt besteht (sich also der Programmeffekt innerhalb und außerhalb des Bereiches mit Common Support systematisch unterscheidet), so ist der geschätzte Programmeffekt verzerrt (LECHNER, 2000). Durch die Untersuchung heterogener Programmeffekte in Abhängigkeit von der Höhe der Programmzahlungen kann diese Inkonsistenz aufgefangen werden (Kapitel 8.4.1).

Die Annahme, dass alle Teilnehmer ein vergleichbares Treatment erhalten, ist in der empirischen Analyse verletzt. So können Betriebe z. B. zwischen verschiedenen Agrarumweltmaßnahmen (Grünlandextensivierung, Vertragsnaturschutz etc.) wählen und einen geringen oder hohen Anteil der LF im Rahmen von AUM bzw. der AZ bewirtschaften. Da die Buchführungsdaten keine Unterscheidung zwischen verschiedenen AUM erlauben und keine Informationen zum Umfang der geförderten Fläche enthalten, können vergleichbare Treatments nicht identifiziert werden. Die Annahme homogener Treatments ist verletzt, 
kann aber teilweise durch die Analyse der Programmeffekte in Abhängigkeit von der Höhe der Programmzahlungen aufgefangen werden.

Indirekte Effekte von Agrarumweltmaßnahmen und der Ausgleichszulage auf nicht teilnehmende Betriebe sind durch SUTVA ausgeschlossen. Die empirische Analyse in den Kapiteln 8.3 und 8.4 zeigt jedoch, dass Agrarumweltmaßnahmen und die Ausgleichszulage den Bodeneinsatz in den teilnehmenden Betrieben erhöhen. Indirekte Effekte auf nicht teilnehmende Betriebe könnten über die Anpassung des Bodenmarktes entstehen.

\subsection{Effekte der Programmteilnahme für Deutschland}

Tabelle 8.3 stellt die Effekte der Teilnahme an Agrarumweltmaßnahmen und der Ausgleichszulage für die gesamtdeutsche Untersuchungsgruppe dar. Die Spalten (1) und (2) zeigen die mittleren Wachstumsraten von Teilnehmern $\left(\Delta y_{i}^{1}\right)$ und Kontrollbetrieben $\left(\Delta y_{j}^{0}\right)$, Spalte (3) die Differenz zwischen beiden, den mittleren Effekt der Programmteilnahme auf die Teilnehmer (ATT). In den Spalten (4) und (5) sind die t-Statistik und die Effektstärke des ATT abgebildet. Ein positiver (negativer) Wert des ATT zeigt, dass Teilnehmer höhere (geringere) Wachstumsraten der Ergebnisvariablen aufweisen als Kontrollbetriebe. Die Resultate aus Tabelle 8.3 sind in Abbildung 8.1 noch einmal graphisch dargestellt.

Teilnehmerbetriebe (AUM, AZ) weisen ein signifikant höheres Flächenwachstum auf als Kontrollbetriebe. Zwischen 2000 und 2005 hat sich die LF von AUM-Teilnehmern (AZTeilnehmern) im Durchschnitt um 7,6 \% (7,8\%) erhöht, während Kontrollbetriebe mit $2,4 \%(5,8 \%)$ ein deutlich geringeres Flächenwachstum aufweisen. Der ATT in Höhe von 5,2\% (AUM) bzw. 2,0\% (AZ) entspricht der Differenz beider Wachstumsraten und ist auf einem Niveau von $1 \%$ (AUM) bzw. $5 \%$ (AZ) signifikant.

Der positive Effekt von Umweltmaßnahmen auf die betriebliche LF ist durch Untersuchungen von Osterburg und Stratmann (2002) sowie durch Pufahl und Weiss (2009) belegt. Das höhere Flächenwachstum der Teilnehmerbetriebe ist vermutlich auf die Flächenbindung der Programmzahlungen zurückzuführen. Eine Ausweitung der förderfähigen Flächen scheint eine Strategie der Gewinnmaximierung zu sein - Betriebe wachsen über die Fläche, nicht über die Erhöhung der Nutzungsintensität. Die Teilnahme an einigen flächenstarken Agrarumweltmaßnahmen (z. B. der Grünlandextensivierung) ist darüber hinaus an eine minimale bzw. maximale Viehbesatzdichte gebunden. Teilnahmewillige Betriebe mit einer zu hohen Viehbesatzdichte pachten in der Regel Fläche zu, um die Viehbesatzdichte je Hauptfutterfläche zu reduzieren und damit die Bewirtschaftungsauflagen von AUM zu erfüllen. 
Tabelle 8.3: $\quad$ Effekte der AUM- und AZ-Teilnahme auf die Teilnehmer (ATT), Greedy Matching, Deutschland gesamt

\begin{tabular}{|c|c|c|c|c|c|c|}
\hline Ergebnisvariable & Einheit & $\begin{array}{c}\text { Teilnehmer } \\
\Delta y_{i}^{1} \\
\text { (1) }\end{array}$ & $\begin{array}{c}\text { Kontrollb. } \\
\Delta y_{j}^{0} \\
(2)\end{array}$ & $\begin{array}{c}\text { ATT } \\
\Delta y_{i}^{1}-\Delta y_{j}^{0} \\
\text { (3) }\end{array}$ & $\begin{array}{c}\text { t-Wert } \\
\text { Signifikanz } \\
\text { (4) }\end{array}$ & $\begin{array}{c}\text { Effektstärke } \\
\qquad d \\
\text { (5) }\end{array}$ \\
\hline \multicolumn{7}{|l|}{ Agrarumweltmaßnahmen } \\
\hline $\ln L F$ & ha & 0,076 & 0,024 & 0,052 & $6,08 * * *$ & 0,22 \\
\hline ln Grünland & ha & 0,055 & $-0,058$ & 0,113 & $4,62 * * *$ & 0,17 \\
\hline In Anteil Pachtland & $\%$ & 0,043 & $-0,017$ & 0,060 & $1,80 *$ & 0,07 \\
\hline $\ln A K$ Betrieb & AKE & $-0,010$ & $-0,016$ & 0,006 & 0,60 & 0,02 \\
\hline $\ln$ AK Betrieb (je 100 ha) & AKE & $-0,086$ & $-0,040$ & $-0,046$ & $-3,90 * * *$ & $-0,14$ \\
\hline ln Außerbetriebliche AK & $\mathrm{h} / \mathrm{Jahr}$ & 0,033 & 0,046 & $-0,013$ & $-0,21$ & $-0,01$ \\
\hline ln Kapital (je ha) & TEuro & 0,040 & 0,065 & $-0,025$ & $-1,33$ & $-0,05$ \\
\hline In Rinderbesatz & GV & $-0,213$ & $-0,174$ & $-0,039$ & $-1,39$ & $-0,05$ \\
\hline In Rinderbesatzdichte & $\mathrm{GV}$ & $-0,112$ & $-0,049$ & $-0,063$ & $-3,72 * * *$ & $-0,14$ \\
\hline ln Ausgaben PSM (je ha) & TEuro & 0,104 & 0,059 & 0,045 & 0,97 & 0,04 \\
\hline ln Ausgaben Dünger (je ha) & TEuro & 0,100 & 0,164 & $-0,064$ & $-2,18 * *$ & $-0,08$ \\
\hline $\ln$ Umsatz & TEuro & 0,066 & 0,046 & 0,020 & 1,12 & 0,04 \\
\hline ln Umsatz (je ha) & TEuro & $-0,007$ & 0,029 & $-0,036$ & $-2,05 * *$ & $-0,08$ \\
\hline \multicolumn{7}{|l|}{ Ausgleichszulage } \\
\hline $\ln L F$ & ha & 0,078 & 0,058 & 0,020 & $2,57 * *$ & 0,09 \\
\hline ln Grünland & ha & 0,047 & $-0,056$ & 0,103 & $4,92 * * *$ & 0,17 \\
\hline ln Anteil Pachtland & $\%$ & 0,024 & $-0,029$ & 0,053 & $1,91 *$ & 0,07 \\
\hline $\ln$ AK Betrieb & AKE & $-0,009$ & $-0,015$ & 0,006 & 0,71 & 0,02 \\
\hline $\ln A K$ Betrieb (je 100 ha) & AKE & $-0,087$ & $-0,073$ & $-0,014$ & $-1,25$ & $-0,04$ \\
\hline In Außerbetriebliche AK & $\mathrm{h} / \mathrm{Jahr}$ & 0,088 & 0,151 & $-0,063$ & $-1,09$ & $-0,04$ \\
\hline ln Kapital (je ha) & TEuro & $-0,016$ & 0,021 & $-0,037$ & $-2,13 * *$ & $-0,07$ \\
\hline ln Rinderbesatz & GV & $-0,140$ & $-0,180$ & 0,040 & 1,62 & 0,06 \\
\hline In Rinderbesatzdichte & GV & $-0,112$ & $-0,046$ & $-0,066$ & $-4,33 * * *$ & $-0,15$ \\
\hline ln Ausgaben PSM (je ha) & TEuro & 0,072 & 0,061 & 0,011 & 0,22 & 0,01 \\
\hline ln Ausgaben Dünger (je ha) & TEuro & 0,138 & 0,160 & $-0,022$ & $-0,78$ & $-0,03$ \\
\hline $\ln U m s a t z$ & TEuro & 0,088 & 0,066 & 0,022 & 1,43 & 0,05 \\
\hline ln Umsatz (je ha) & TEuro & 0,010 & 0,016 & $-0,006$ & $-0,39$ & $-0,01$ \\
\hline
\end{tabular}

1. Sterne kennzeichnen die statistische Signifikanz im T-Test für die Gleichheit der Mittelwerte auf einem Niveau von $1 \% * * *, 5 \% * *$ und $10 \% *$.

2. Effektstärke: sehr klein $|d|<0,2$, klein $0,2 \leq|d|<0,5$, mittel $0,5 \leq|d|<0,8$, groß $0,8 \leq|d|$.

Quelle: eigene Berechnungen, Datenquellen vgl. Tabellen A2 und A3.

Tabelle 8.3 zeigt weiter, dass das Flächenwachstum primär über die Zupacht von Flächen erfolgt. Teilnehmerbetriebe weisen ein um 6,0\% (AUM) bzw. 5,3\% (AZ) signifikant höheres Wachstum des Pachtflächenanteils auf als Kontrollbetriebe. Teilnehmer wachsen zu etwa gleichen Teilen über Grünland- und Ackerflächen, während Kontrollbetriebe fast ausschließlich über Ackerflächen wachsen. Dementsprechend nimmt das Grünland in AUM-Betrieben (AZ-Betrieben) um 5,5\% (4,7\%) zu, während dieses in Kontrollbetrieben um 5,8 \% (5,6 \%) abnimmt. Der Effekt der Programmteilnahme bezogen auf die Grün- 
landfläche beträgt $+11,3 \%$ für Agrarumweltmaßnahmen und $+10,3 \%$ für die Ausgleichszulage. Die Irrtumswahrscheinlichkeit ist für beide Effekte kleiner $1 \%$.

Abbildung 8.1: Effekte der AUM- und AZ-Teilnahme auf die Teilnehmer (ATT), Greedy Matching, Deutschland gesamt

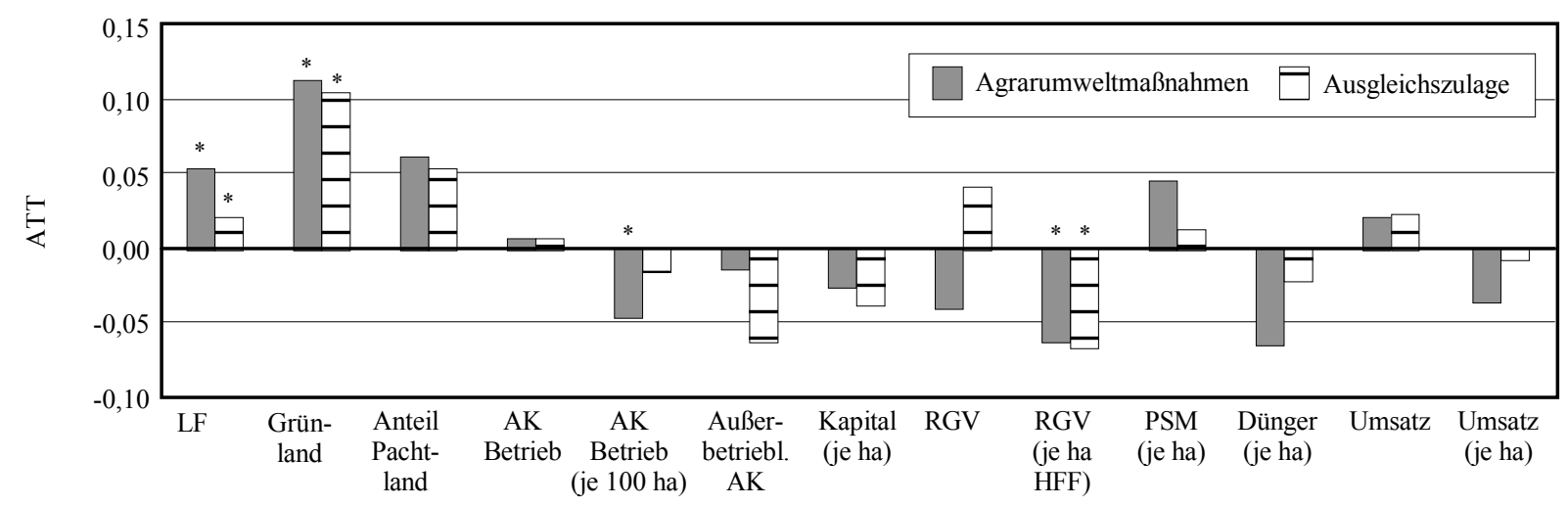

1. Die Balken stellen die Unterschiede der prozentualen Wachstumsraten (2000 bis 2005) zwischen Teilnehmern und Kontrollbetrieben dar.

2. Ein Stern (*) kennzeichnet die statistische Signifikanz des ATT im T-Test für die Gleichheit der Mittelwerte auf einem Niveau von $<5 \%$ und einer Effektstärke von $\mathrm{d}>0,1$.

Quelle: eigene Berechnungen, Datenquellen vgl. Tabellen A2 und A3.

Bezogen auf die analysierten Betriebe (2000 bis 2005) hat die Programmteilnahme zu einer Zunahme des Grünlandes von durchschnittlich $+1,65$ ha (AUM) bzw. +0,19 ha (AZ) je Betrieb geführt. Die Grünlandzunahme in Teilnehmerbetrieben dürfte auf verschiedene Ursachen zurückzuführen sein: Die Programmzahlungen verringern die Nutzungskosten des Grünlandes und verbessern seine innerbetriebliche Wettbewerbsfähigkeit, z. B. gegenüber Futtermais (vgl. Osterburg, 2005: 12). Für die im Rahmen der GAK umgesetzten Agrarumweltmaßnahmen besteht ein explizites Verbot des Grünlandumbruchs (nur Grünlandextensivierung) bzw. das Gebot zum Erhalt des Umfangs des betrieblichen Grünlandes (BMVEL, 2000). Inwieweit die betrachteten Programme zum Erhalt des Grünlandes insgesamt beitragen, kann aus dieser Analyse nicht geschlossen werden.

Der allgemein rückläufige Trend der landwirtschaftlichen Arbeitskräfte spiegelt sich im Rückgang der betrieblichen Arbeitskräfte (AK Betrieb) in den Teilnehmer- und Kontrollbetrieben wider. Ein signifikanter Einfluss der AUM- bzw. AZ-Teilnahme auf die Entwicklung der betrieblichen Arbeitskräfte ist nicht zu beobachten. Vergleichbare Ergebnisse anderer Studien liegen der Autorin nicht vor. In Übereinstimmung mit GooDWIN et al. (2007) zeigen die Ergebnisse in Tabelle 8.3 keinen signifikanten Zusammenhang zwischen der außerbetrieblichen Erwerbsbeteiligung des Betriebsleiterpaares (Außerbetriebliche $A K)$ und der Teilnahme an AUM bzw. an der AZ. Hieraus ist zu schließen, dass die Teilnahme an Agrarumweltmaßnahmen und der Ausgleichszulage in Deutschland weder mit 
Substitutions- noch mit Gewinneffekten, bezogen auf die Arbeitszeitallokation, verbunden ist.

Infolge des Flächenwachstums verringert sich der flächenbezogene Arbeitseinsatz ( $A K$ Betrieb je $100 \mathrm{ha}$ ) in den Teilnehmerbetrieben stärker als in den Kontrollbetrieben. Die Anzahl der Arbeitskräfte je 100 ha reduziert sich in AUM-Betrieben um 8,6\%, während diese in den Kontrollbetrieben nur um 4,0 \% abnehmen. Der ATT in Höhe von $-4,6 \%$ ist hochsignifikant. Die Ausgleichszulage hat ebenfalls einen tendenziell negativen Einfluss auf den flächenbezogenen Arbeitseinsatz; der Effekt ist jedoch nicht statistisch signifikant.

Ein zentrales Ziel von Agrarumweltmaßnahmen ist die Reduzierung der Nutzungsintensität landwirtschaftlicher Flächen. Als Indikatoren hierfür werden die Viehbesatzdichte und der Einsatz mineralischer Pflanzenschutz- und Düngemittel je Hektar verwendet. Im Gegensatz dazu ist die Ausgleichszulage an keine nennenswerten Umweltauflagen gebunden. Alle Teilnehmer- und Kontrollbetriebe (AUM, AZ) weisen einen vergleichbaren Rückgang des Rinderbestandes auf. Die Rinderbesatzdichte je Hauptfutterfläche (RGV je HFF) verringert sich jedoch in den Teilnehmerbetrieben (AUM, AZ) wesentlich stärker (je $11,2 \%)$ als in den Kontrollbetrieben $(-4,9 \%,-4,6 \%)$. Der ATT, bezogen auf die Rinderbesatzdichte, beträgt für AUM (AZ) -6,3\% (-6,6\%). Der Effekt ist in beiden Fällen statistisch hochsignifikant. $\mathrm{Zu}$ einem vergleichbaren Ergebnis kommt OSTERBURG (2005) für den Untersuchungszeitraum 1990/1991 bis 1998/1999 für Deutschland.

Die AUM-Teilnahme ist mit einer signifikanten Reduzierung der Düngemittelausgaben je Hektar LF (Ausgaben Dünger je ha) ${ }^{51}$ um 6,4 \% verbunden. Unter der Annahme, dass ausschließlich Stickstoffdünger ${ }^{52}$ verwendet wird, entspricht dies einer Reduzierung von etwa $9 \mathrm{~kg}$ Stickstoff je Hektar LF. Für die AZ ist kein vergleichbarer Effekt zu beobachten. Beide untersuchten Programme haben keinen signifikanten Einfluss auf Ausgaben für Pflanzenschutzmittel je Hektar (Ausgaben PSM je ha). Ursachen für den verhältnismäßig geringen Effekt der AUM-Teilnahme auf die Reduzierung des Dünge- und Pflanzenschutzmittelaufwand können sein:

51 Die Einkaufspreise (ohne Umsatzsteuer) sind im Zeitraum 2000 bis 2004 für Düngemittel um 15 Prozentpunkte gestiegen und für Pflanzenschutzmittel um etwa 10 Prozentpunkte gefallen (Statistisches Bundesamt, div. Jgg.). Es wird unterstellt, dass die Preisentwicklung Teilnehmerund Kontrollbetriebe gleichermaßen trifft.

52 Kalkammonsalpeter (26\% Stickstoff, Schüttgut), Preis je 100 kg Nährstoff: 49,00 Euro (2000) bzw. 62,01 Euro (2005) (EUROSTAT, 2006). 
- AUM werden häufig von Betrieben in Anspruch genommen, deren Flächennutzungsintensität bereits vor Programmbeginn den Teilnahmevoraussetzungen entspricht.

- Inputreduzierende AUM auf Ackerflächen werden, mit Ausnahme des hier nicht betrachteten ökologischen Landbaus, kaum in Anspruch genommen.

- Viele AUM sind nicht auf die stoffliche Inputreduzierung ausgerichtet sondern reduzieren die mechanische Nutzungsintensität (Mulch- und Direktsaatverfahren) bzw. deren zeitliche Taktung (späte Mahd).

Das deutliche Flächenwachstum infolge der AUM-Teilnahme hat, im Vergleich zu Kontrollbetrieben, keinen Effekt auf die Umsatzentwicklung. Dies ist vermutlich auf den signifikanten Rückgang des Umsatzes je Hektar zurückzuführen (ATT=-3,6 \%), der wiederum in Zusammenhang mit der Reduzierung der Viehbesatzdichte und der Düngemittelausgaben je Hektar stehen dürfte. Für AZ-Teilnehmer sind keine signifikanten Effekte hinsichtlich des betrieblichen und flächenbezogenen Umsatzes festzustellen.

Die in Tabelle 8.3, Spalte (5) ausgewiesenen Effektstärken des ATT sind entsprechend den Orientierungswerten von COHEN (1988) als sehr klein $(d<0,2)$ und klein $(d \geq 0,2)$ einzustufen. Bei der Beurteilung der Relevanz des ATT ist zu berücksichtigen, dass AUM und AZ hohe Akzeptanzraten aufweisen und darüber hinaus, im Gegensatz zu Flächenprämien, sehr selektiv auf bestimmte Betriebsgruppen wirken.

\section{Vergleich alternativer Matching Schätzer}

Um die Robustheit der Ergebnisse in Tabelle $8.3 \mathrm{zu}$ überprüfen, wurden die Ergebnisse des Greedy Matching mit denen dreier alternativer Schätzer (fixer Caliper Schätzer, Kernel Schätzer und naive Schätzer ${ }^{53}$ ) verglichen. Insbesondere der Kernel Schätzer könnte den unerwünscht hohen Ausschluss von Teilnehmerbeobachtungen ohne vergleichbaren Kontrollbetrieb (vgl. Tabelle 8.2) reduzieren und so zu einer Verringerung der hierauf zurückzuführenden Ergebnisverzerrung führen.

Tabelle 8.4 stellt die Ergebnisse der vier Matching Schätzer vergleichend dar. Durch das Greedy Matching werden alle signifikanten Unterschiede zwischen den Teilnehmer- und Kontrollbetrieben beseitigt. Mit wenigen Ausnahmen (Rinderbesatz, Umsatz, Umsatz je Hektar) trifft dies auch für die Ergebnisse des Caliper Schätzers zu. Mit Hilfe des Kernel Schätzers kann der Selektionseffekt in der Stichprobe nicht kontrolliert werden; auch nach dem Matching bestehen signifikante Unterschiede zwischen den Merkmalen von Teilnehmer- und Kontrollbetrieben.

Der fixe Caliper Schätzer und der Kernel Schätzer sind in Kapitel 7.1.2 beschrieben. Der naive Schätzer berücksichtigt alle potenziellen Teilnehmer- und Kontrollbetriebe, ist also ein DiD-Schätzer „ohne Matching“. 
Tabelle 8.4: Effekte der AUM- und AZ-Teilnahme auf die Teilnehmer (ATT), Vergleich verschiedener Matching Schätzer, Deutschland gesamt

\begin{tabular}{|c|c|c|c|c|c|}
\hline & \multirow[t]{2}{*}{ Einheit } & \multicolumn{4}{|c|}{ ATT verschiedener Schätzer } \\
\hline & & Greedy & Caliper & Kernel & Naive \\
\hline Agrarumweltmaßnahmen & & ATT & ATT & ATT & ATT \\
\hline $\ln L F$ & ha & $0,052 * * *$ & $0,067 * * *$ & $0,103 * * *$ & $0,045 * * *$ \\
\hline ln Grünland & ha & $0,113 * * *$ & $0,157 * * *$ & $0,168 * * *$ & $0,096 * * *$ \\
\hline ln Anteil Pachtland & $\%$ & $0,060 *$ & 0,059 & $0,151 *$ & $\mathbf{0 , 0 3 3} * * *$ \\
\hline $\ln$ AK Betrieb & AKE & 0,006 & $0,037 * * *$ & $0,103 * * *$ & $\mathbf{0 , 0 0 7} *$ \\
\hline ln AK Betrieb (je 100 ha) & AKE & $-0,046 * * *$ & $-0,030 *$ & $-0,001$ & $\mathbf{- 0 , 0 3 8} * * *$ \\
\hline In Außerbetriebliche AK & $\mathrm{h} / \mathrm{Jahr}$ & $-0,013$ & $-0,003$ & 0,019 & $\mathbf{- 0 , 0 6 1} * * *$ \\
\hline ln Kapital (je ha) & TEuro & $-0,025$ & $-0,053 *$ & $-0,193 * * *$ & $-0,079 * * *$ \\
\hline ln Rinderbesatz & GV & $-0,039$ & $\mathbf{0 , 0 1 2}$ & $-0,109$ & $\mathbf{0 , 0 5 2} * * *$ \\
\hline ln Rinderbesatzdichte & GV & $-0,063 * * *$ & $-0,079 * * *$ & $-0,296 * * *$ & $-0,032 * * *$ \\
\hline ln Ausgaben PSM (je ha) & TEuro & 0,045 & $0,132 * *$ & $0,175 *$ & $\mathbf{- 0 , 0 5 1} * * *$ \\
\hline ln Ausgaben Dünger (je ha) & TEuro & $-0,064 * *$ & $-0,047$ & $-0,063$ & $\mathbf{0 , 0 2 8} * *$ \\
\hline $\ln$ Umsatz & TEuro & 0,020 & $\mathbf{0 , 0 2 7}$ & 0,060 & $0,056 * * *$ \\
\hline ln Umsatz (je ha) & TEuro & $-0,036 * *$ & $-0,057 * *$ & $-0,074 *$ & 0,005 \\
\hline \multicolumn{6}{|l|}{ Ausgleichszulage } \\
\hline $\ln L F$ & ha & $0,020 * *$ & $0,057 * *$ & 0,090 & $\mathbf{0 , 0 3 6} * * *$ \\
\hline ln Grünland & ha & $0,103 * * *$ & 0,086 & $0,103 *$ & $\mathbf{0 , 0 8 3} * * *$ \\
\hline ln Anteil Pachtland & $\%$ & $0,053 *$ & $-0,014$ & 0,009 & $\mathbf{0 , 0 2 9} * *$ \\
\hline $\ln A K$ Betrieb & $\mathrm{AKE}$ & 0,006 & $-0,017$ & $-0,003$ & $\mathbf{0 , 0 1 3} * * *$ \\
\hline ln AK Betrieb (je 100 ha) & AKE & $-0,014$ & $-0,074 * *$ & $-0,093 * * *$ & $\mathbf{- 0 , 0 2 3} * * *$ \\
\hline In Außerbetriebliche AK & $\mathrm{h} / \mathrm{Jahr}$ & $-0,063$ & 0,181 & $-0,048$ & $-\mathbf{0 , 0 9 7} * * *$ \\
\hline ln Kapital (je ha) & TEuro & $-0,037 * *$ & $-0,028$ & $-0,069$ & $\mathbf{- 0 , 0 8 5} * * *$ \\
\hline In Rinderbesatz & GV & 0,040 & 0,121 & $\mathbf{0 , 1 7 7} * *$ & $\mathbf{0 , 1 3 4} * * *$ \\
\hline In Rinderbesatzdichte & GV & $-0,066 * * *$ & $-0,007$ & $-0,005$ & $-0,018 * * *$ \\
\hline In Ausgaben PSM (je ha) & TEuro & 0,011 & $-0,198 *$ & $\mathbf{- 0 , 2 5 0} * *$ & $\mathbf{0 , 0 3 5} * *$ \\
\hline ln Ausgaben Dünger (je ha) & TEuro & $-0,022$ & $-0,224$ & $-0,234 * * *$ & $\mathbf{0}$ \\
\hline $\ln$ Umsatz & TEuro & 0,022 & 0,033 & $-0,055$ & $0,055 * * *$ \\
\hline $\ln$ Umsatz (je ha) & TEuro & $-0,006$ & $-0,030$ & 0,040 & $\mathbf{0 , 0 1 2} * *$ \\
\hline
\end{tabular}

1. Fett gedruckte Werte kennzeichnen die statistische Signifikanz im T-Test für die Gleichheit der Mittelwerte vor dem Matching auf einem Niveau von $>5 \%$.

2. Sterne kennzeichnen die statistische Signifikanz im T-Test für die Gleichheit der Mittelwerte nach dem Matching auf einem Niveau von $1 \% * * *, 5 \% * *$ und $10 \% *$.

Quelle: eigene Berechnungen, Datenquellen vgl. Tabellen A2 und A3.

Der Unterschied zwischen den Ergebnissen des Greedy bzw. des Caliper Schätzers und dem Kernel Schätzer ist auf die sehr unterschiedlichen Schätzansätze zurückzuführen: Der Greedy und der Caliper Schätzer definieren eine maximal zulässige Distanz (Caliper) zwischen den Teilnehmer- und Kontrollbetrieben. Teilnehmerbeobachtungen, für die keine Kontrollbetriebe innerhalb des Calipers aufzufinden sind, werden von der Analyse ausgeschlossen. Der Kernel Schätzer verwendet einen Teil der Kontroll- 
beobachtungen, um für jeden Teilnehmer einen hypothetischen Kontrollbetrieb zu konstruieren. Dieses Verfahren hat den Vorteil, dass kein Teilnehmer von der Analyse ausgeschlossen wird. Nachteilig ist, dass für Teilnehmer mit extrem abweichenden Merkmalen (Propensity Scores) kein ähnlicher Kontrollbetrieb konstruiert werden kann, da nicht genügend viele Informationen für die Situation ohne Programmteilnahme vorliegen. In der Konsequenz können die Unterschiede zwischen den Merkmalen der Teilnehmer- und denen der Kontrollbetriebe nicht vollständig kontrolliert werden.

Die Konsistenz des Greedy Schätzers kann nur im Vergleich zum Caliper Schätzer beurteilt werden, da nur für diese beiden Fälle der Selektionseffekt (aufgrund beobachteter Merkmale) kontrolliert werden konnte. Die Vorzeichen, das Niveau und die Signifikanz des Programmeffektes beider Schätzer stimmen weitgehend überein. In der Tendenz sind die Programmeffekte des Caliper Schätzers für Agrarumweltmaßnahmen etwas höher und für die Ausgleichszulage etwas geringer als die des Greedy Schätzers.

\subsection{Heterogenität der Effekte der Programmteilnahme}

In der bisherigen Analyse wurde von einer binären Entscheidung - Teilnahme oder Nichtteilnahme - ausgegangen. Tatsächlich unterscheidet sich die Programmteilnahme zwischen Betrieben hinsichtlich des flächenmäßigen Teilnahmeumfangs. $\mathrm{Zu}$ vermuten ist, dass die Höhe der Programmeffekte umso stärker ist, je mehr Fläche ein Betrieb im Rahmen von Agrarumweltmaßnahmen bzw. der Ausgleichszulage bewirtschaftet. Weiterhin werden Betriebe mit unterschiedlichen Charakteristika variierende Strategien zur Anpassung des betrieblichen Managements an die Bedingungen der Programmteilnahme wählen. Schließlich variieren die geographischen und politischen Rahmenbedingungen innerhalb Deutschlands, so dass regional unterschiedliche Effekte der Programmteilnahme zu erwarten sind.

Die folgende Ergebnisdarstellung konzentriert sich auf jene Effekte, die deutlich von denen der gesamtdeutschen Analyse (vgl. Tabelle 8.3) abweichen, eine Irrtumswahrscheinlichkeit kleiner $5 \%$ und eine Effektstärke größer 0,1 aufweisen.

\subsubsection{Effekte der Programmteilnahme in Abhängigkeit von der Höhe der Programmzahlungen}

Die Programmzahlungen können als Maß für die flächenmäßige „Intensität“ der AUMund AZ-Teilnahme interpretiert werden. Zur Berücksichtigung des Skaleneffektes werden die vom Betrieb erhaltenen Programmzahlungen auf die betriebliche LF bezogen. Der Umfang der tatsächlich geförderten Fläche sowie die Prämienhöhe der geförderten Fläche sind nicht bekannt (vgl. Kapitel 6.1). Aus Tabelle 8.2 geht hervor, dass die selektierten AUM- 
und AZ-Teilnehmer deutlich geringere Programmzahlungen je Hektar LF aufweisen als nicht selektierte (von der Analyse ausgeschlossene) Teilnehmer. Hieraus ergibt sich die Frage, ob die geschätzten Effekte der Programmteilnahme in Tabelle 8.3 durch den Ausschluss von Teilnehmern mit hohen Programmzahlungen verzerrt gemessen wurden.

Eine Antwort hierauf gibt Abbildung 8.2: Die Richtung der Programmeffekte (positiv, negativ) wird nicht durch die Höhe der AUM- und AZ-Zahlungen je Hektar beeinflusst. Ein positiver Zusammenhang dagegen ist zwischen dem Niveau des Programmeffektes und der Höhe der Programmzahlungen je Hektar zu erkennen: je höher die AUM- bzw. AZ-Zahlungen je Hektar, desto höher ist das Niveau des Programmeffektes. Dieser Zusammenhang ist für die AZ weniger deutlich ausgeprägt als für AUM. Dementsprechend ist davon auszugehen, dass die in Tabelle 8.3 präsentierten Effekte der Programmteilnahme tendenziell unterschätzt werden.

Abbildung 8.2: $\quad$ Effekte der AUM- und AZ-Teilnahme auf die Teilnehmer (ATT) differenziert nach Programmzahlungen je Hektar, Greedy Matching
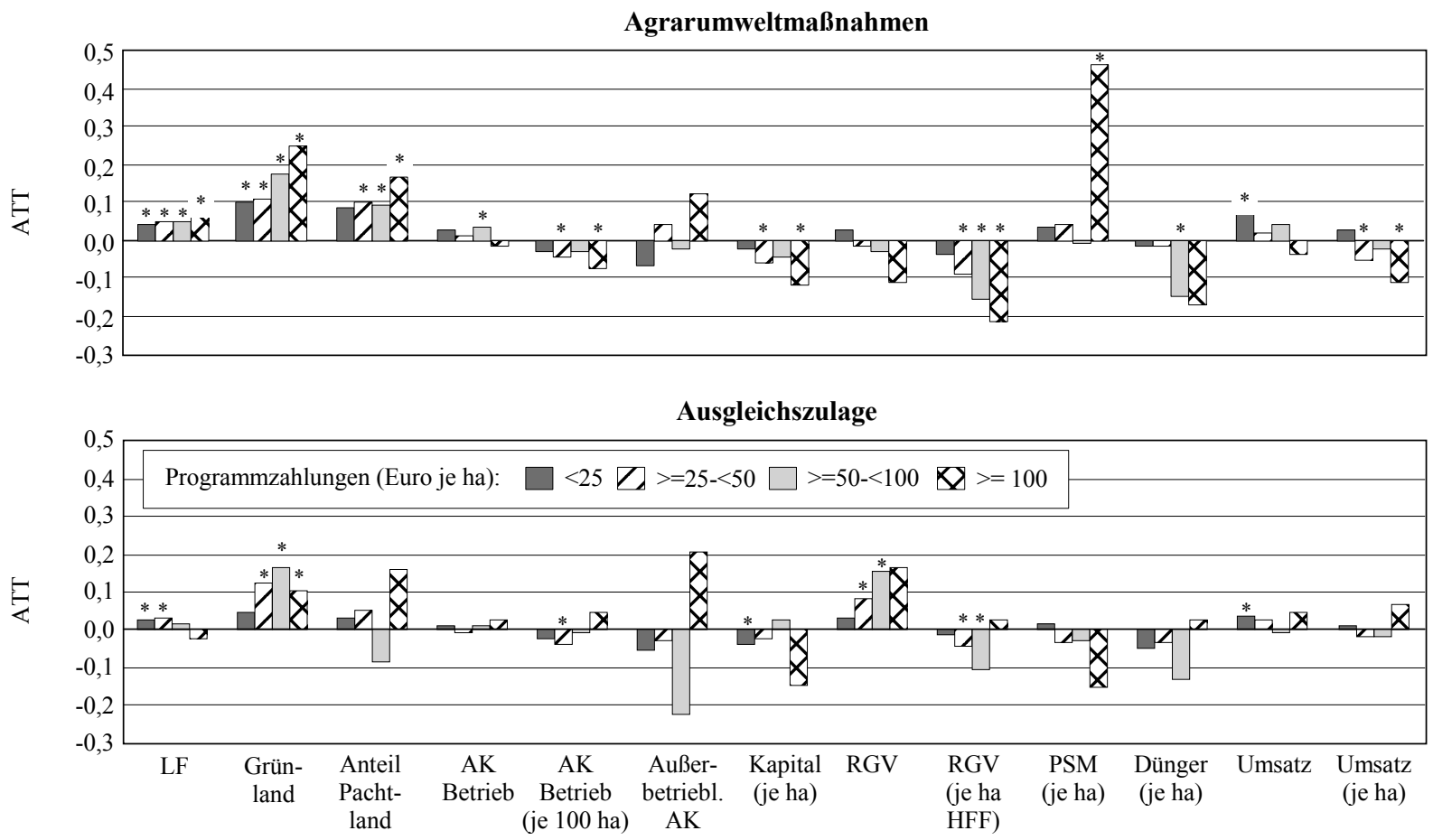

1. Die Balken stellen die Unterschiede der prozentualen Wachstumsraten (2000 bis 2005) zwischen Teilnehmern und Kontrollbetrieben dar.

2. Ein Stern (*) kennzeichnet die statistische Signifikanz im T-Test für die Gleichheit der Mittelwerte auf einem Niveau von $<5 \%$ und einer Effektstärke von $\mathrm{d}>0,1$.

Quelle: eigene Berechnungen, Datenquellen vgl. Tabellen A2 und A3. 
Ein Zusammenhang zwischen der Höhe der Programmzahlungen und dem Programmeffekt besteht insbesondere für die Merkmale, für die in Tabelle 8.3 signifikante Effekte zu beobachten waren ( $L F$, Grünland, RGV je HFF, Dünger je Hektar). So steigt z. B. der Effekt der AUM-Teilnahme bezogen auf das Grünland von $+9,6 \%$ in der Gruppe mit geringen Programmzahlungen $(<25$ Euro/ha) auf $+24,7 \%$ in der Gruppe mit Programmzahlungen über 100 Euro/ha. Die Effektstärke erhöht sich dementsprechend von $d=0,1$ $(<25$ Euro/ha) auf d=0,4 ( $>100$ Euro/ha). Der positive Effekt der AZ-Teilnahme auf den Rinderbesatz (RGV) nimmt mit steigenden Programmzahlungen ebenfalls zu. Ein positiver Zusammenhang zwischen der Höhe der Programmzahlungen und dem Viehbesatz ist auch durch SHAIK und HELMERS (2006) belegt.

Merkmale, für die in Tabelle 8.3 kein eindeutiger Effekt der Programmteilnahme festzustellen war, ändern sich mit zunehmenden Programmzahlungen nur marginal oder weisen eine große Varianz in Abhängigkeit von der Höhe der Programmzahlungen auf (AUM: PSM je Hektar, AZ: Außerbetriebliche AK). AUM-Teilnehmer mit Programmzahlungen von über 100 Euro je Hektar verzeichnen, im Vergleich zu Kontrollbetrieben, eine um 46,2 \% höhere Zunahme der PSM-Ausgaben je Hektar. Die Irrtumswahrscheinlichkeit dieses Effektes liegt unter $1 \%$. Eine mögliche Erklärung hierfür könnten gestiegene Ausgaben für Pflanzenschutzmittel infolge der Anwendung von Mulch- und Direktsaatverfahren sein. Weiterhin ist eine Verschiebung der Nutzungsintensität von den geförderten hin zu den nicht geförderten Flächen, z. B. von Grünland auf Ackerland, denkbar.

\subsubsection{Effekte der Programmteilnahme nach Regionen}

Abbildung 8.3 zeigt die Effekte der Programmteilnahme, differenziert für Betriebe in Nord-, West-, Süd- und Ostdeutschland. Regionale Unterschiede zwischen den Effekten der AUM- und AZ-Teilnahme sind aufgrund der unterschiedlichen agrarstrukturellen Situation in den verschiedenen Teilen Deutschlands zu erwarten. Auch die Umsetzung von Agrarumweltmaßnahmen und der Ausgleichszulage unterscheidet sich deutlich zwischen den standörtlich begünstigten Regionen in Nordwestdeutschland (Region Nord: Schleswig-Holstein, Niedersachen) und Bundesländern mit eher unterdurchschnittlichen natürlichen Standortvoraussetzungen (Region Süd: Bayern, Baden-Württemberg) (vgl. Kapitel 2). Bei der Interpretation der Ergebnisse ist zu berücksichtigen, dass die Bundesländer innerhalb der Regionen nicht gleichmäßig repräsentiert sind. ${ }^{54}$

\footnotetext{
54 Die Region Nord wird von Betrieben aus Niedersachsen dominiert, West durch Betriebe aus Nord-
} rhein-Westfalen. In der Region Ost fehlen Betriebe aus Sachsen. 
Abbildung 8.3: $\quad$ Effekte der AUM- und AZ-Teilnahme auf die Teilnehmer (ATT), differenziert nach Regionen, Greedy Matching
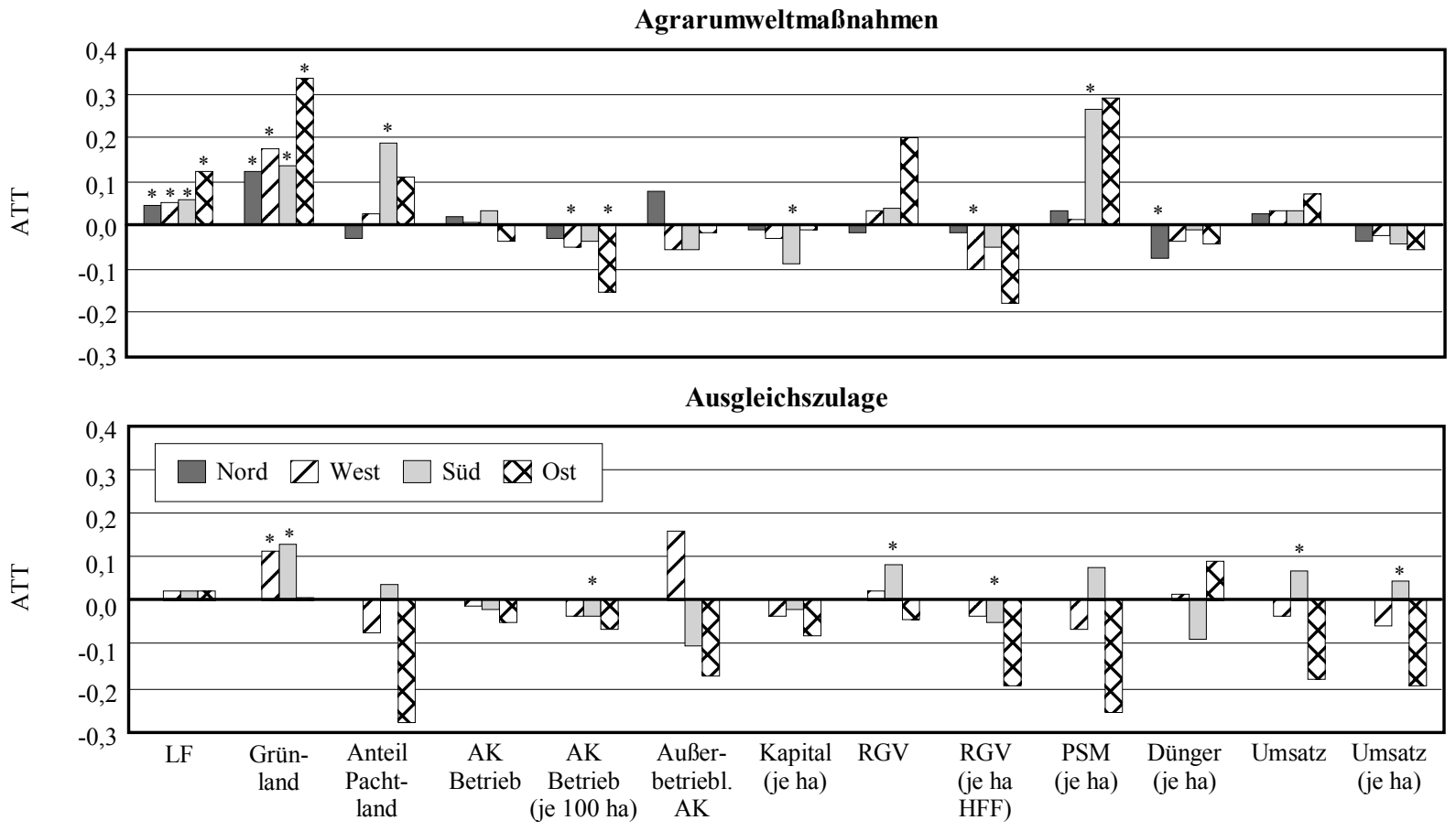

1. Die Balken stellen die Unterschiede der prozentualen Wachstumsraten (2000 bis 2005) zwischen Teilnehmern und Kontrollbetrieben dar.

2. Ein Stern (*) kennzeichnet die statistische Signifikanz im T-Test für die Gleichheit der Mittelwerte auf einem Niveau von $<5 \%$ und einer Effektstärke mit $\mathrm{d}>0,1$.

3. Aufgrund der geringen Anzahl an AZ-Teilnehmern in Schleswig-Holstein und Niedersachsen sind für die Ausgleichszulage, Region Nord keine Ergebnisse dargestellt.

Quelle: eigene Berechnungen, Datenquellen vgl. Tabellen A2 und A3.

Der für Gesamtdeutschland ermittelte positive Effekt der AUM- und AZ-Teilnahme auf das Flächenwachstum, insbesondere auf das Grünland, findet sich auch in der regionalen Analyse bestätigt. Der Effekt von Agrarumweltmaßnahmen auf das Flächen- und Grünlandwachstum ist in Ostdeutschland am größten und in Norddeutschland am geringsten. Der Effekt der AZ-Teilnahme auf das Flächenwachstum ist, in Analogie zum gesamtdeutschen Ergebnis, geringer als der der AUM-Teilnahme und nicht statistisch signifikant. Deutliche regionale Unterschiede sind hinsichtlich des Grünlandwachstums zu beobachten: Während west- und süddeutsche AZ-Betriebe einen signifikant positiven Effekt der Programmteilnahme bezogen auf das Grünland aufweisen, ist dieser Effekt in Ostdeutschland nahe Null.

Der negative Einfluss der AUM-Teilnahme auf den flächenbezogenen Arbeitseinsatz ( $A K$ Betrieb je 100 ha) spiegelt sich auch in den Ergebnissen der regionalen Analyse wider. Dieser Effekt ist in west- und ostdeutschen AUM-Betrieben signifikant negativ. 
Die Programmteilnahme (AUM, AZ) hat in allen Regionen einen negativen Effekt auf die Kapitalausstattung der teilnehmenden Betriebe, wenngleich dieser Effekt nur in Süddeutschland (AUM) mit -8,6 \% statistisch signifikant ist. Diese Entwicklung könnte darauf hindeuten, dass Teilnehmerbetriebe auf (kapital- und arbeits-)extensive Bewirtschaftungsmethoden umstellen, entweder, um die langfristige Rentabilität des Betriebes zu sichern und/oder um betriebliches Kapital nichtlandwirtschaftlichen Nutzungen zuführen zu können.

Die Reduzierung der Nutzungsintensität als Folge von Agrarumweltmaßnahmen fällt regional sehr unterschiedlich aus. Eine signifikante Verringerung der Ausgaben für Düngemittel je Hektar (Dünger je Hektar, ATT= -6,9 \%) zeigten AUM-Teilnehmer in Norddeutschland. In den übrigen Regionen ist dieser der Effekt nahe Null. Die für Gesamtdeutschland beobachtete Reduzierung der Rinderbesatzdichte ( $R G V$ je $H F F)$ ist nur in Westdeutschland statistisch signifikant (ATT=-9,8\%). Beide Effekte sind ein Anzeichen dafür, dass eine Nutzungsextensivierung im Zuge der AUM-Teilnahme vorrangig in Nord- und Westdeutschland stattfindet. Ein Grund hierfür könnte das vergleichsweise hohe Ausgangsniveau der Nutzungsintensität in diesen Regionen sein. Die Ausgaben für Pflanzenschutzmittel je Hektar haben in den süddeutschen AUM-Betrieben, absolut und im Vergleich zu Kontrollbetrieben, signifikant zugenommen (ATT $=+26,3 \%)$. Dies könnte auf eine Verschiebung der Nutzungsintensität von geförderten Flächen (z. B. Grünland) auf nicht geförderte Flächen (z. B. Acker) hindeuten.

Für die Ausgleichszulage sind in Süddeutschland ebenfalls statistisch signifikante Effekte auf die Nutzungsintensität zu beobachten. Einerseits geht der Rinderbesatz in Teilnehmerbetrieben deutlich langsamer zurück als in den Kontrollbetrieben $(\mathrm{ATT}=+8,0)$. Die Ausgleichszulage verlangsamt demnach den allgemeinen Trend des Rückgangs der Rinderbestände. Andererseits reduziert sich infolge der AZ-Teilnahme - und der damit verbundenen Flächenausweitung - die Rinderbesatzdichte geringfügig (ATT= $-4,1 \%$ ). Aus ökologischer Sicht ist der Erhalt des Rinderbestandes dann positiv zu beurteilen, wenn Mindestbesatzdichten ( 0,3 bis $0,5 \mathrm{RGV}$ je HFF) bzw. Höchstbesatzdichten (1,0 bis 1,4 RGV je HFF) erreicht bzw. nicht überschritten werden. Von den analysierten süddeutschen AZFutterbaubetrieben $(n=651)$ liegen jedoch $88 \%$ oberhalb und $6 \%$ unterhalb dieses Grenzwertes, so dass der ökologische Nutzen der Reduzierung des Rinderbesatzes vermutlich als gering einzustufen ist.

Der signifikant positive Effekt der AZ-Teilnahme auf den Umsatz (ATT=6,2 \%) bzw. den Umsatz je Hektar (ATT=4,4 \%) dürfte auf das Flächenwachstum und den den Rinderbesatz erhaltenden Effekt der Ausgleichszulage zurückzuführen sein. Die Umsatzsteigerung kann als Produktionseffekt, die Umsatzsteigerung je Hektar als Produktivitätseffekt interpretiert werden. 


\subsubsection{Effekte der Programmteilnahme nach Betriebsformen}

Abbildung 8.4 stellt die Effekte der Programmteilnahme differenziert nach Ackerbau-, Futterbau-, Veredlungs- und Gemischtbetrieben dar. Aufgrund der inhaltlichen Ausrichtung von Agrarumweltmaßnahmen und der Ausgleichszulage auf Grünland und standörtlich benachteiligte Regionen stellen Futterbaubetriebe den größten Anteil aller Teilnehmer, gefolgt von Ackerbaubetrieben. Dieses Verhältnis spiegelt sich auch in den Stichprobengrößen wider: Unter den selektierten AUM-Teilnehmern (AZ-Teilnehmern) finden sich 1.003 (853) Futterbaubetriebe und 610 (203) Ackerbaubetriebe. Zur Beurteilung der Effekte der AUM- und AZ-Teilnahme sind Veredlungs- und Gemischtbetriebe nur von nachrangiger Bedeutung, da diese Betriebe nur einen geringen Anteil an der Grundgesamtheit geförderter Betriebe repräsentieren.

Abbildung 8.4: Effekte der AUM- und AZ-Teilnahme auf die Teilnehmer (ATT), differenziert nach Betriebsformen, Greedy Matching
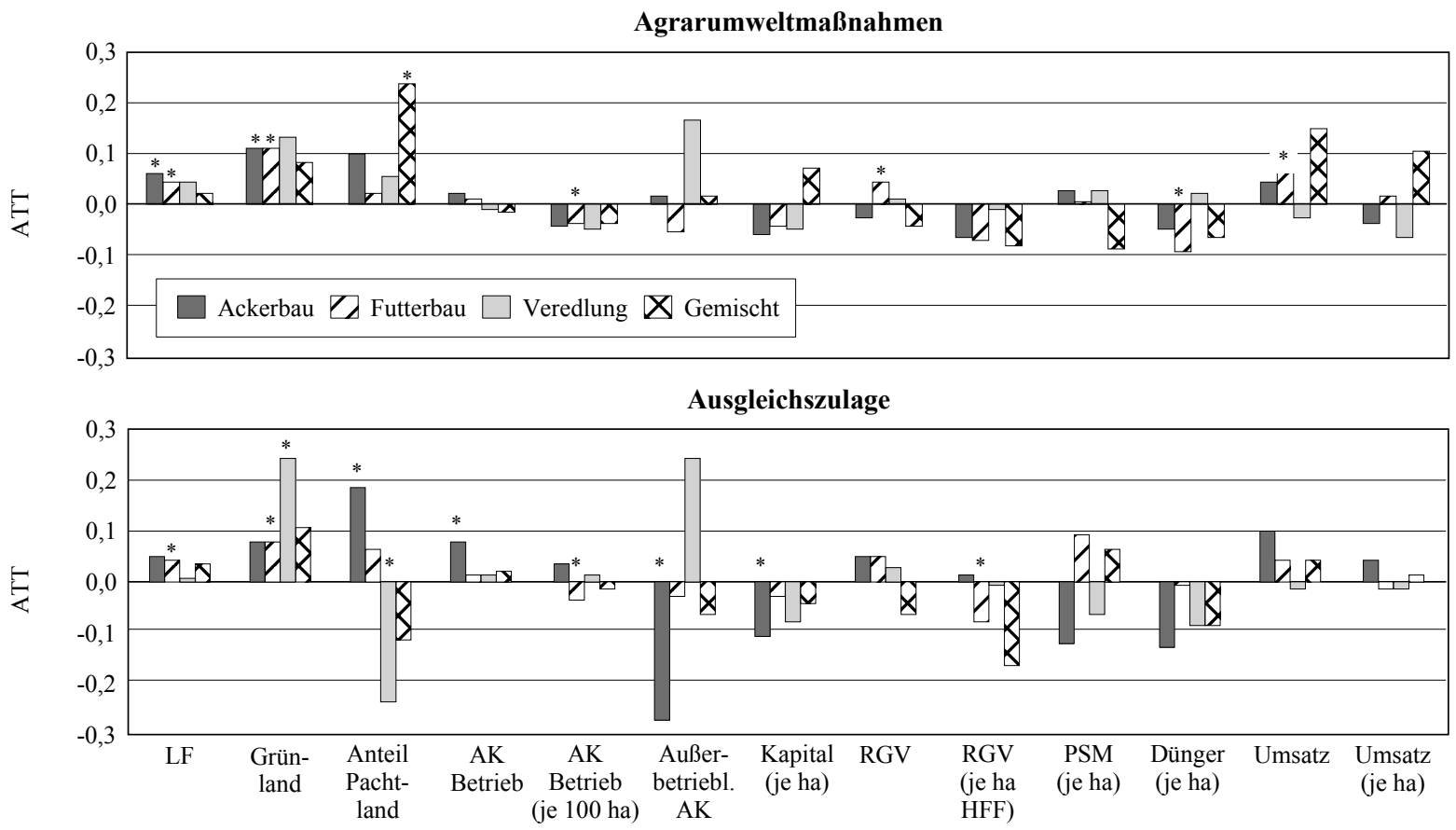

1. Die Balken stellen die Unterschiede der prozentualen Wachstumsraten (2000 bis 2005) zwischen Teilnehmern und Kontrollbetrieben dar.

2. Ein Stern (*) kennzeichnet die statistische Signifikanz im T-Test für die Gleichheit der Mittelwerte auf einem Niveau von $<5 \%$ und einer Effektstärke von $\mathrm{d}>0,1$.

Quelle: eigene Berechnungen, Datenquellen vgl. Tabellen A2 und A3. 
Der positive Effekt der Programmteilnahme (AUM, AZ) auf das Flächenwachstum bestätigt sich auch in den betriebsformspezifischen Ergebnissen. Ackerbaubetriebe weisen tendenziell ein etwas höheres Flächenwachstum auf als Futterbaubetriebe, da der Boden in Ackerbaubetrieben ein vergleichsweise wichtigerer Produktionsfaktor ist als in Futterbaubetrieben. Lediglich Veredlungsbetriebe mit AZ-Teilnahme weisen mit $+23,9 \%$ eine noch höhere Zunahme der Grünlandfläche auf. Aufgrund der geringen Grünlandfläche in Veredlungsbetrieben entspricht dieser Wert jedoch lediglich einer absoluten Zunahme von durchschnittlich 0,2 ha Grünland je Betrieb.

Die Reduzierung des flächenbezogenen Arbeitseinsatzes (AK Betrieb je $100 \mathrm{LF}$ ) infolge des höheren Flächenwachstums von Teilnehmerbetrieben betrifft insbesondere Futterbaubetriebe mit Teilnahme an Agrarumweltmaßnahmen (ATT=-3,4\%) bzw. an der Ausgleichszulage (ATT $=-3,0 \%$ ). Die AZ-Teilnahme von Ackerbaubetrieben hat dagegen einen positiven Effekt auf den betrieblichen Arbeitseinsatz (AK Betrieb) in Höhe von $+7,2 \%$ bei gleichzeitigem Rückgang der außerbetrieblichen Erwerbsarbeit des Betriebsleiterpaares $(\mathrm{ATT}=27,6 \%)$. Außerbetriebliche Arbeit wird durch betriebliche Arbeit substituiert, da infolge der AZ-Teilnahme das Wertgrenzprodukt der betrieblichen Arbeit steigt (Substitutionseffekt).

Für Futterbaubetriebe mit Programmteilnahme (AUM, AZ) ist eine signifikante Reduzierung der Rinderbesatzdichte $(R G V$ je $H F F)$ zu beobachten. Die Ergebnisse auf gesamtdeutscher und regionaler Ebene bestätigen dies. Der für Süddeutschland beobachtete, den Rinderbesatz erhaltende Effekt der Ausgleichszulage (vgl. Abbildung 8.3) wird durch die betriebsformspezifischen Auswertungen in Abbildung 8.4 nicht bestätigt. Eine signifikante Verringerung der Düngemittelausgaben je Hektar ist nur für AUM-Futterbaubetriebe zu beobachten. Überraschend ist, dass - trotz der Verringerung der Düngemittelausgaben die Produktivität (Umsatz je Hektar) nahezu unverändert bleibt. Dies könnte ein Indiz für die im Zuge der AUM-Teilnahme gestiegene Stickstoffeffizienz sein. OsTERBURG (2007: 292 ff.) zeigt, dass Stickstoff-Effizienzreserven in Futterbaubetrieben vorhanden sind. Die AZ-Teilnahme hat in keiner Betriebsform einen signifikanten Einfluss auf die Entwicklung des PSM- und Düngemitteleinsatzes je Hektar bzw. auf die Produktionsmenge (Umsatz).

Das signifikant höhere Umsatzwachstum von Futterbaubetrieben mit AUM-Teilnahme ist vermutlich auf das Flächenwachstum zurückzuführen. Die Programmteilnahme (AUM, AZ) hat in allen Betriebsformen (Ausnahme: AUM-Gemischtbetriebe) einen negativen Effekt auf die Kapitalausstattung teilnehmender Betriebe. Signifikant ist dieser Effekt lediglich in AZ-Ackerbaubetrieben (ATT $=-10 \%$ ). 


\subsubsection{Effekte der Programmteilnahme nach Betriebsgröße}

Abbildung 8.5 stellt den kausalen Effekt der Programmteilnahme für Betriebe unterschiedlicher Betriebsgrößen (LF) dar. Eine Abhängigkeit des betrieblichen Flächenwachstums von der Betriebsgröße ist für Agrarumweltmaßnahmen nicht zu erkennen. Große Betriebe (> 100 ha) mit AZ-Teilnahme scheinen schneller zu wachsen als AZ-Teilnehmer mit einer Betriebsgröße unter 100 ha LF.

Abbildung 8.5: Effekte der AUM- und AZ-Teilnahme auf die Teilnehmer (ATT), differenziert nach Betriebsgröße (ha LF), Greedy Matching
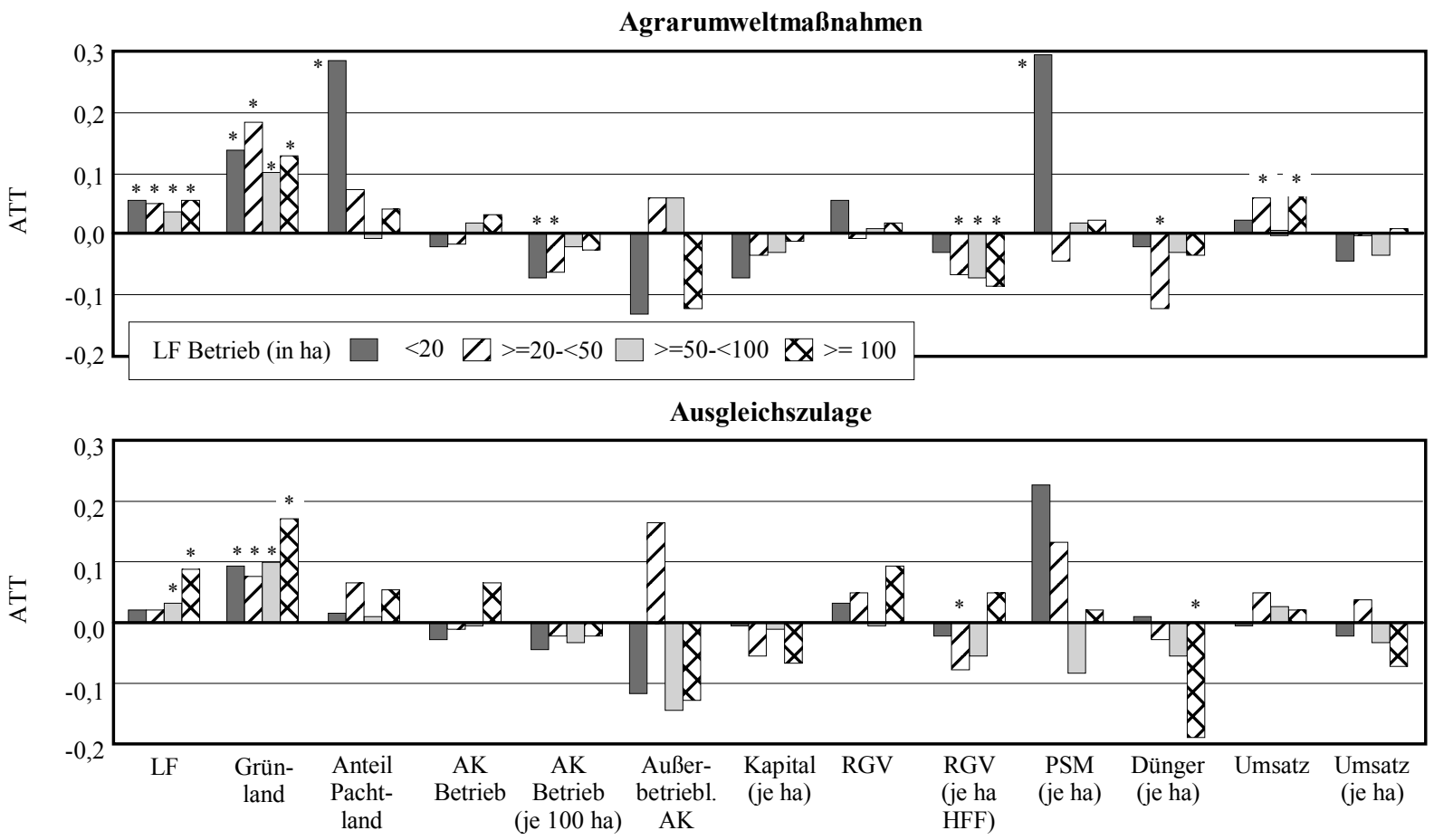

1. Die Balken stellen die Unterschiede der prozentualen Wachstumsraten (2000 bis 2005) zwischen Teilnehmern und Kontrollbetrieben dar.

2. Ein Stern $\left(^{*}\right)$ kennzeichnet die statistische Signifikanz im T-Test für die Gleichheit der Mittelwerte auf einem Niveau von $<5 \%$ und einer Effektstärke von $\mathrm{d}>0,1$.

Quelle: eigene Berechnungen, Datenquellen vgl. Tabellen A2 und A3.

In Betrieben mit AUM-Teilnahme nimmt der negative Beschäftigungseffekt je Hektar ( $A K$ Betrieb je 100 ha) mit zunehmender Betriebsgröße ab. Der ATT des flächenbezogenen Arbeitseinsatzes beträgt in kleinen Betrieben $(<20$ ha LF) $-6,9 \%$ und in großen Betrieben ( $>100$ ha LF) nur noch -2,2\%. Mögliche Erklärungsansätze hierfür sind, dass große Betriebe mehr Alternativen für die Verwendung der freigesetzten Arbeitskraft haben als kleine Betriebe oder große Betriebe bereits vor der Programmteilnahme relativ arbeitskraftextensiv wirtschafteten. 
Mit steigender Betriebsgröße nimmt der negative Effekt der AUM-Teilnahme auf die Rinderbesatzdichte $(R G V$ je $H F F)$ zu. Der entsprechende Programmeffekt beträgt in kleinen Betrieben $(<20$ ha LF) Null und in großen Betrieben $(>100$ ha LF) -8,0 \%. Die stärkere Reduzierung der Rinderbesatzdichte in großen Betrieben ist auf den geringeren (absoluten) Rinderbesatz zurückzuführen, der bei einem vergleichbaren Flächenwachstum zu einer stärkeren Reduzierung der Besatzdichte führt als in kleinen Betrieben.

Für AZ-Teilnehmer ist eine Zunahme des Flächenwachstums mit steigender Betriebgröße zu beobachten. Während die LF in kleinen Betrieben $(<20$ ha LF) um 1,4 \% wächst sind, liegt die Wachstumsrate von großen Betrieben ( $>100$ ha LF) bei $8,1 \%$. Der positive Zusammenhang zwischen der Betriebsgröße und dem Flächenwachstum findet sich erneut bestätigt. In Analogie zum Flächenwachstum verringern sich die Ausgaben für Düngemittel je Hektar in großen AUM-Betrieben deutlich (ATT=-18,4 \%), während diese in kleinen Betrieben konstant bleiben.

\subsubsection{Effekte der Programmteilnahme in Abhängigkeit von der Teil- nahmewahrscheinlichkeit}

Aufgrund der Ausprägung betrieblicher Merkmale kann jedem Betrieb eine Wahrscheinlichkeit der Programmteilnahme zugeordnet werden. So hat z. B. ein flächen- und grünlandstarker Futterbaubetrieb mit niedriger Viehbesatzdichte eine hohe Wahrscheinlichkeit an Agrarumweltmaßnahmen teilzunehmen, da dieser Betrieb die Teilnahmebedingungen bereits erfüllt (vgl. Tabelle 8.1). Ein teilnahmewilliger Betrieb mit einer zu hohen Viehbesatzdichte muss dagegen erst betriebliche Anpassungen vornehmen, die mit Kosten und Organisationsaufwand verbunden sind. Demzufolge ist zu vermuten, dass Betriebe mit einer hohen Wahrscheinlichkeit der Programmteilnahme den höchsten Nutzen aus der Teilnahme ziehen.

Abbildung 8.6 stellt die Ergebnisänderung $\left(\Delta y_{i}^{1}, \Delta y_{j}^{0}\right)$ von Teilnehmern (durchgezogene Linie) und Kontrollbetrieben (gestrichelte Linie) in Abhängigkeit von der Wahrscheinlichkeit der AUM-Teilnahme $(p(X))$ für die Ergebnisvariablen mit signifikanten Effekten in der gesamtdeutschen Analyse dar (vgl. Tabelle 8.3). Der Effekt der Programmteilnahme auf die Teilnehmer (ATT) entspricht der Fläche zwischen beiden Linien. Da nichtparametrische Schätzungen in Bereichen geringer Dichte unzuverlässig sein können, ist durch den Kerndichteplot das Intervall mit hinreichend vielen Beobachtungen gekennzeichnet. 
Abbildung 8.6: $\quad$ Einfluss der bedingten Wahrscheinlichkeit der AUM-Teilnahme $p(X)$ auf die Ergebnisänderung $\Delta y_{i}^{1}, \Delta y_{j}^{0}$ (nicht-parametrische Regression), Deutschland gesamt

In LF

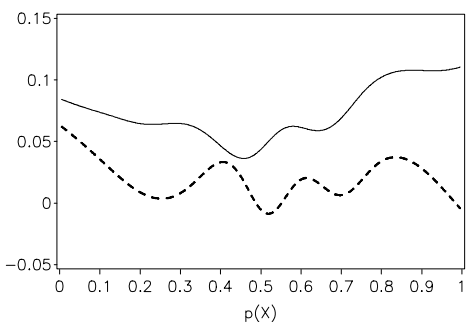

In RGV/HFF

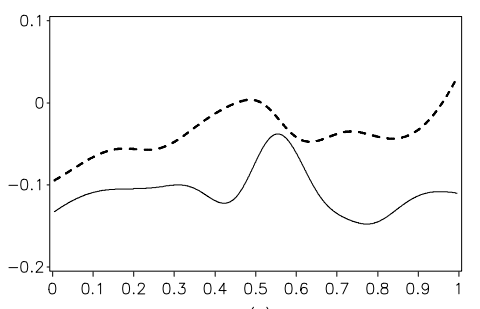

$\mathrm{D}(\mathrm{X})$

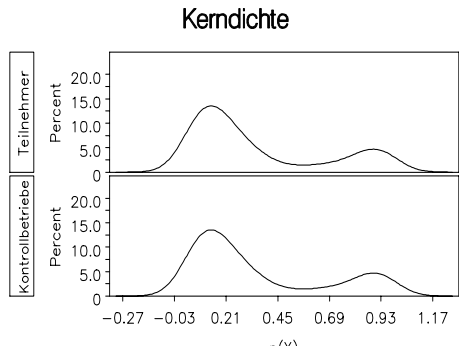

In Gruenland

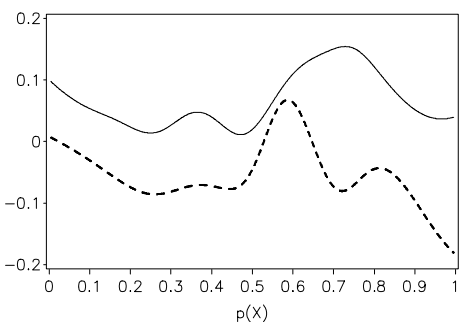

In Duenger (je ha)

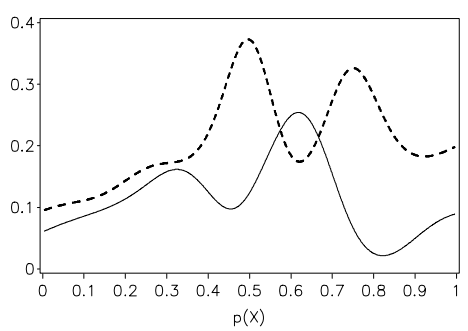

In AK Betrieb (je 100 ha)

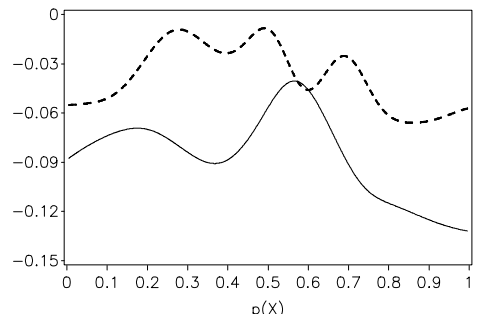

In Umsatz (je ha)

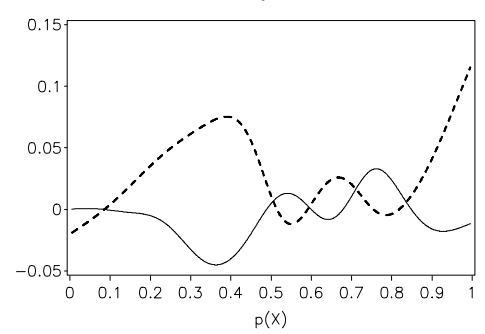

1. Nadaraya-Watson Schätzer mit Gauß'scher Kernfunktion und optimaler Bandbreite.

2. Plots für die übrigen Ergebnisvariablen sowie für die Ausgleichszulage vgl. Abbildungen A2 und A3.

Quelle: eigene Berechnungen, Datenquellen vgl. Tabellen A2 und A3.

Aus Abbildung 8.6 ist ersichtlich, dass mit zunehmender Wahrscheinlichkeit der Teilnahme der Programmeffekt auf das Flächen- und Grünlandwachstum, den flächenbezogenen AK-Einsatz (AK Betrieb je 100 ha) und die Rinderbesatzdichte (RGV/HFF) ebenfalls zunimmt. Der Zusammenhang zwischen der Wahrscheinlichkeit der Programmteilnahme und den Düngemittelausgaben je Hektar sowie dem Umsatz je Hektar ist weniger eindeutig. Vergleichbare Aussagen wie für Agrarumweltmaßnahmen gelten auch für die Ausgleichszulage (vgl. Abbildung A3 im Anhang). 


\subsection{Zusammenfassung}

Die Analyse des Propensity Score Matching zeigt einen signifikant positiven Effekt der Teilnahme an Agrarumweltmaßnahmen und an der Ausgleichzulage auf das betriebliche Flächenwachstum, insbesondere auf das betriebliche Grünland. Teilnehmerbetriebe an Agrarumweltmaßnahmen weisen ein um 5,2 \% höheres Flächenwachstum bzw. ein um 11,3 \% höheres Grünlandwachstum auf als vergleichbare Kontrollbetriebe. Infolge des Flächenwachstums verringert sich bei der AUM-Teilnahme der flächenbezogene Arbeitseinsatz, die Rinderbesatzdichte sowie der Düngemittelaufwand je Hektar signifikant. Die Programmeffekte der Ausgleichszulage bleiben deutlich hinter denen von Agrarumweltmaßnahmen zurück.

Das höhere Flächenwachstum der Teilnehmerbetriebe ist vermutlich auf die Flächenbindung der Programmzahlungen zurückzuführen. Eine Ausweitung der förderfähigen Flächen scheint eine Strategie der Gewinnmaximierung zu sein - Betriebe wachsen über die Fläche statt über die Intensivierung des Faktoreinsatzes. Der positive Effekt der Programmteilnahme auf das Flächenwachstum ist ein Indiz für den komparativen Vorteil von Teilnehmerbetrieben am Bodenmarkt gegenüber Betrieben ohne Teilnahme an den Programmen.

Die Höhe der Effekte der Teilnahme an Agrarumweltmaßnahmen und an der Ausgleichszulage variiert in Abhängigkeit von der Höhe der Programmzahlungen. Das Niveau des Programmeffektes nimmt mit steigenden Programmzahlungen je Hektar zu. Ein positiver Zusammenhang ist auch zwischen dem Niveau des Programmeffektes und der Wahrscheinlichkeit der Programmteilnahme zu beobachten: Betriebe, deren Programmteilnahme aufgrund ihrer betrieblichen Merkmale sehr wahrscheinlich ist, weisen die höchsten Programmeffekte auf. Die Effekte der AUM- und AZ-Teilnahme variieren eher unsystematisch in Abhängigkeit von der Region, der Betriebsform und der Betriebsgröße. 


\section{Empirische Ergebnisse der Panelregressionen}

Im Kapitel 9 werden verschiedene Regressionsmodelle (gepoolte OLS-Modell, FixedEffekt-Modell, GMM-Modell mit orthogonalen Abweichungen, System GMM-Modell) verwendet, um den Effekt der Teilnahme an Agrarumweltmaßnahmen und der Ausgleichszulage auf das betriebliche Flächenwachstum zu ermitteln. Das gepoolte OLS-Modell und das Fixed-Effekt-Modell dienen als Referenz, um das Intervall eines relativ unverzerrten GMM-Schätzers eingrenzen zu können (vgl. BoND, 2002: 5). Die empirische Spezifikation der Modelle ist in Kapitel 9.1 dargestellt. Im Kapitel 9.2 werden die Modelle hinsichtlich ihrer Annahmen und Ergebnisse miteinander verglichen. Zur Analyse heterogener Effekte der Programmteilnahme werden die genannten Modelle um Interaktionsterme erweitert bzw. zu einem Selektionsmodell modifiziert (Kapitel 9.3). Die Ergebnisse der Panelanalyse sind in Kapitel 9.4 zusammengefasst.

\subsection{Empirische Spezifikation der verwendeten Modelle}

In der parametrischen Analyse wird nur der Effekt der Teilnahme an Agrarumweltprogrammen und an der Ausgleichszulage auf das betriebliche Flächenwachstum untersucht. Das empirische Modell wird in Anlehnung an die theoretischen Überlegungen zu den Bestimmungsfaktoren des betrieblichen Flächeneinsatzes spezifiziert. Für stetig skalierte Variablen mit ausschließlich positiven Werten wird ein log-log-linearer Zusammenhang mit dem interessierenden Ergebnis unterstellt. ${ }^{55}$

Die Analyse beginnt mit einem statischen, gepoolten OLS-Modell (POLS I). Die abhängige Variable, $\ln L F_{i r f t}$, ist der natürliche Logarithmus (ln) der landwirtschaftlich genutzte Fläche eines Betriebes $i$, mit der Betriebsform $f$ ( $f=$ Ackerbau, Futterbau, Veredlung, Gemischt), in der Region $r$ ( $r=$ Kreis $^{1}, \ldots$, Kreis $\left.^{n}\right)$ im Jahr $t(t=2001, \ldots, 2005)$ :

$$
\begin{aligned}
\ln L F_{i r f t}= & \gamma_{0}+\gamma_{1} \ln \text { Kap }_{i r f, t-1}+\gamma_{2} \ln A K_{i r f, t-1}+\gamma_{3} \ln D Z_{i r f, t-1}+ \\
& \gamma_{4} \text { Gew }_{i r f, t-1}+\gamma_{5} A U M_{i r f t}+\gamma_{6} A Z_{i r f t}+\beta_{1} \text { Alter }_{i r f t}+ \\
& \beta_{2} \ln \text { Pacht }_{r t}+\beta_{3} \text { Preis }_{r t}+\beta_{4} \ln M L F_{r t}+ \\
& \omega_{f}+c_{r}+\mu_{t}+u_{i r f t}, \quad t=2001, \ldots, 2005 .
\end{aligned}
$$

55 Alternative Modellspezifikationen (linear, linear-log, log-linear) wurden anhand der Summe der Residuenquadrate mit der gewählten log-log-linearen Spezifikation verglichen und aufgrund eines geringeren Erklärungsgehaltes verworfen. Für das Alter des Betriebsleiters $\left(\right.$ Alter $_{\text {irft }}$ ) sowie den Erzeugerpreisindex $\left(\right.$ Preis $\left._{r t}\right)$ wird zunächst ein linearer Zusammenhang unterstellt. 
Der Bodeneinsatz des Betriebes wird durch den Kapital- und Arbeitseinsatz ( $\ln \operatorname{Kap}_{i r f, t-1}$, $\left.\ln A K_{i r f, t-1}\right)$, die Summe der erhaltenen Direktzahlungen ( $\ln D Z_{i r f, t-1}$ ) und den bereinigten Betriebsgewinn $^{56}\left(\right.$ Gew $\left._{\text {irf }, t-1}\right)$ beeinflusst. Da diese Regressoren aufgrund simultaner Kausalität vermutlich endogen sind, werden jeweils die Vorjahreswerte $(t-1)$ dieser Regressoren verwendet. Betriebsspezifische Merkmale mit einem Einfluss auf die Produktionsentscheidung landwirtschaftlicher Betriebe, wie z. B. das Alter des Betriebsleiters $\left(\text { Alter }_{i r f t}\right)^{57}$ und die Betriebsform $\left(\omega_{f}\right)$, werden durch entsprechende Variablen kontrolliert. Die Charakteristika des regionalen Boden- und Produktmarktes sowie der regionalen Agrarstruktur können aufgrund der Datenlage nur kreisspezifisch abgebildet werden. Als Kontrollvariablen für diese Faktoren werden der Pachtpreis für landwirtschaftliche Flächen ( $\ln$ Pacht $\left.t_{r t}\right)$, der Erzeugerpreisindex ( Preis $_{r t}$ ) sowie die durchschnittliche Flächenausstattung landwirtschaftlicher Betriebe $\left(\ln M L F_{r t}\right)$ in einem Kreis verwendet. Der Einfluss unbeobachteter, regions- und jahresspezifischer Einflussfaktoren, z. B. der natürlichen Standortbedingungen für die landwirtschaftliche Produktion und gesamtwirtschaftliche Schocks, werden durch regions- und jahresspezifische Dummyvariablen $\left(c_{r}, \mu_{t}\right)$ kontrolliert.

Die binäre Entscheidung zur Teilnahme an Agrarumweltprogrammen und der Ausgleichszulage wird durch die Variablen $A U M_{i r f t}$ bzw. $A Z_{i r f t}$ abgebildet. ${ }^{58}$ Die entsprechenden Koeffizienten $\gamma_{5}$ und $\gamma_{6}$ repräsentieren $c . p$., den marginalen Effekt der AUM- und AZTeilnahme auf das betriebliche Flächenwachstum.

Im POLS I ist die vermutlich wichtigste Determinante der betrieblichen Flächenausstattung - die Flächenausstattung des Betriebes im Vorjahr - nicht enthalten. Um die Qualität der Schätzung zu verbessern, wird ein dynamisches Modell POLS II spezifiziert.

$$
\begin{aligned}
\ln L F_{i r f t}= & \alpha_{0}+\alpha_{1} \ln L F_{i r f, t-1}+\alpha_{2} \ln L F_{i r f, t-2}+\gamma_{1} \ln \operatorname{Kap}_{i r f, t-1}+ \\
& \gamma_{2} \ln A K_{i r f, t-1}+\gamma_{3} \ln D Z_{i r f, t-1}+\gamma_{4} \text { Gew }_{i r f, t-1}+\gamma_{5} A U M_{i r f t}+ \\
& \gamma_{6} A Z_{i r f t}+\beta_{1} \text { Alter }_{i r f t}+\beta_{2} \ln \text { Pacht }_{r t}+\beta_{3} \text { Preis }_{r t}+ \\
& \beta_{4} \ln M L F_{r t}+\omega_{f}+c_{r}+\mu_{t}+u_{i r f t}, \quad t=2002, \ldots, 2005 .
\end{aligned}
$$

56 Der Betriebsgewinn wurde um produktionsbezogene Direktzahlungen, um die Ausgleichszulage sowie um Zahlungen im Rahmen von Agrarumweltmaßnahmen bereinigt.

57 Die schulische und berufliche Qualifikation des Betriebsleiters hatte keinen signifikanten Einfluss auf den Flächeneinsatz und wurde in der Modellspezifikation berücksichtigt.

58 Durch die binäre Skalierung von $A U M_{i r f t}$ bzw. $A Z_{i r f t}$ gehen Informationen verloren, da sich die Teilnehmer durch unterschiedlich hohe Programmzahlungen unterscheiden. Der Einfluss der Höhe der Programmzahlungen auf das betriebliche Flächenwachstum wird in Kapitel 9.3.1 untersucht. 
Im POLS II werden, zusätzlich zu den im POLS I verwendeten Regressoren, die Vorjahreswerte der betrieblichen Flächenausstattung ( $\left.\ln L F_{i r f, t-1}, \ln L F_{i r f, t-2}\right)$ als Determinanten der aktuellen betrieblichen LF herangezogen. Statt der üblichen AR1-Spezifikation wird eine AR2-Spezifikation mit zwei Lags der abhängigen Variable verwendet. Dies ist notwendig, um die dynamische Vollständigkeit des Modells sicherzustellen und eine Autokorrelation der Fehler zu unterbinden (WoOLDRIDGE 2002: 173 ff.). Ein Nachteil der AR2Spezifikation ist, dass hierdurch die ersten zwei Untersuchungsjahre $(2000,2001)$ für die empirische Analyse verloren gehen. Die Berücksichtigung der Flächenausstattung des Vorjahres ist auch für die Kontrolle des Selektionseffektes relevant, da die Wahrscheinlichkeit der AUM-Teilnahme mit steigender LF eines Betriebes zunimmt (vgl. Tabelle $8.1)$.

Der Effekt der Programmteilnahme wird im POLS I und POLS II vermutlich verzerrt gemessen, da der Einfluss unbeobachteter, betriebsspezifischer Faktoren nicht explizit kontrolliert wird. Die Kontrolle dieser Einflussfaktoren ist, unter den in Kapitel 7.2.1.2 genannten Annahmen, durch ein Fixed-Effekt-Modell (FE I) möglich.

$$
\begin{aligned}
\ln L F_{i r f t}= & \gamma_{0}+\gamma_{1} A U M_{i r f t}+\gamma_{2} A Z_{i r f t}+\beta_{1} \ln \text { Pacht }_{r t}+\beta_{2} \text { Preis }_{r t}+ \\
& \beta_{3} \ln M L F_{r t}+\eta_{i}+\mu_{t}+v_{i r f t}, \quad t=2000, \ldots, 2005
\end{aligned}
$$

Modell FE I misst den Effekt der AUM- und AZ-Teilnahme $\left(A U M_{i r f t}, A Z_{i r f t}\right)$ auf das jährliche betriebliche Flächenwachstum, kontrolliert für den Einfluss kreisspezifischer Pachtund Erzeugerpreise $\left(\ln\right.$ Pacht $_{r t}$, Preis $\left._{r t}\right)$, der kreisspezifischen Agrarstruktur $\left(\ln M L F_{r t}\right)$ sowie jahresspezifischer Schocks $\left(\mu_{t}\right)$. Der unbeobachtete, betriebsspezifische Effekt, $\eta_{i}$, ist mit einer betriebsspezifischen Dummyvariable vergleichbar.

Das FE I beruht auf der Annahme strikter Exogenität ${ }^{59}$ aller Regressoren. Potenziell endogene Regressoren, wie z. B. der betriebliche Arbeits- und Kapitaleinsatz, die Höhe der Direktzahlungen und der bereinigte Betriebsgewinn, sind im FE I daher nicht enthalten. Im FE I fehlen darüber hinaus zeitkonstante regions- und betriebsformspezifische Dummyvariablen. Der Einfluss dieser Regressoren kann, aufgrund der within Transformation der Daten, im FE I nicht kontrolliert werden. ${ }^{60}$ Methodisch bedingt fehlen im FE I wichtige Determinanten des betrieblichen Faktoreinsatzes und der Programmteilnahme. Aus diesem

59 Im Modell berücksichtigte Regressoren dürfen nicht mit gegenwärtigen, zukünftigen und vergangenen Realisationen des Fehlers $v_{\text {irft }}$ korrelieren.

60 Auf die Berücksichtigung von Interaktionstermen regions- bzw. betriebsformspezifischer Dummyvariablen mit Jahresdummies wurde verzichtet, da hierdurch der Erklärungsgehalt des Modells nicht erhöht wurde. Das Alter des Betriebsleiters korreliert nach der within Transformation mit dem jahresspezifischen Effekt und wird ebenfalls nicht berücksichtigt. 
Grund wird der Effekt der AUM- und AZ-Teilnahme im FE I vermutlich verzerrt gemessen.

Unter der weniger strengen Annahme sequentieller Endogenität ${ }^{61}$ können im FE-Modell auch endogene Regressoren berücksichtigt werden. Das entsprechend modifizierte FE IModell hat die Form

$$
\begin{aligned}
\ln L F_{i r f t}= & \beta_{0}+\alpha_{1} \ln L F_{i r f, t-1}+\alpha_{2} \ln L F_{i r f, t-2}+\gamma_{1} \ln \operatorname{Kap}_{i r f, t-1}+ \\
& \gamma_{2} \ln A K_{i r f, t-1}+\gamma_{3} \ln D Z_{i r f, t-1}+\gamma_{4} \text { Gew }_{i r f, t-1}+\gamma_{5} A U M_{i r f t}+ \\
& \gamma_{6} A Z_{i r f t}+\beta_{1} \ln \operatorname{Pacht}_{r t}+\beta_{2} \text { Preis }_{r t}+\beta_{3} \ln M L F_{r t}+ \\
& \eta_{i}+\mu_{t}+v_{i r f t}, \quad t=2002, \ldots, 2005 .
\end{aligned}
$$

Modell FE II ist dem dynamischen POLS II sehr ähnlich und unterscheidet sich nur durch die zusätzliche Berücksichtigung des unbeobachteten betriebsspezifischen Effektes $\eta_{i}$ und das Fehlen zeitkonstanter Regressoren. Die Annahme sequentieller Exogenität kann die Korrelation des Fehlers mit endogenen Regressoren nur reduzieren, in der Regel jedoch nicht unterbinden. Eine andere Möglichkeit zum Umgang mit endogenen Regressoren ist deren Instrumentierung durch Variablen, die mit dem Fehler unkorreliert sind.

Dieses Prinzip wird in den verwendeten GMM-Modellen mit orthogonalen Abweichungen (GMM-OR) und im System GMM-Modell (GMM-SYS) umgesetzt. Das verwendete GMM-OR hat die Form,

$$
\begin{aligned}
\ln L F_{i r f t}= & \alpha_{1} \ln L F_{i r f, t-1}+\alpha_{2} \ln L F_{i r f, t-2}+\gamma_{1} \ln \text { Kap }_{i r f t}+ \\
& \gamma_{2} \ln A K_{i r f t}+\gamma_{3} \ln D Z_{i r f t}+\gamma_{4} \text { Gew }_{i r f t}+\gamma_{5} A U M_{i r f t}+ \\
& \gamma_{6} A Z_{i r f t}+\beta_{1} \text { Alter }_{i r f t}+\beta_{2} \ln \text { Pacht }_{r t}+\beta_{3} \text { Preis }_{r t}+ \\
& \beta_{4} \ln M L F_{r t}+\mu_{i}+\eta_{t}+v_{i r f t}, \quad t=2003, \ldots, 2005 .
\end{aligned}
$$

Im Unterschied zum POLS II und FE II werden im GMM-OR nicht die Vorjahreswerte potenziell endogener Regressoren, sondern die gegenwärtigen Realisationen dieser Regressoren ( $\left.\ln K a p_{i r f t}, \ln A K_{i r f t}, \ln D Z_{i r f t}, G e w_{i r f t}\right)$ berücksichtigt. Um den unbeobachteten, betriebsspezifischen Effekt, $\eta_{i}$, zu kontrollieren, erfolgt die Schätzung auf Basis orthogonaler Abweichungen. ${ }^{62}$ Wie die FE-Modelle enthält das GMM-OR daher keine zeitkonstanten Regressoren. Aufgrund der Datentransformation, der Verwendung zweier Lags der

61 Im Modell berücksichtigte Regressoren dürfen mit den zukünftigen Werten des Fehlers korrelieren, jedoch nicht mit gegenwärtigen und vergangenen Werten des Fehlers.

62

Die orthogonale Abweichung einer Variablen entspricht dem Mittelwert aller verbleibenden, zukünftigen Realisationen dieser Variablen subtrahiert von ihrem gegenwärtigen Wert (vgl. Kapitel 7.2.1.3). 
abhängigen Variable und deren Instrumentierung durch frühere Lags gehen die ersten drei Untersuchungszeitpunkte für die empirische Analyse verloren.

Das GMM-OR hat zwei Schwachstellen: Erstens können zeitkonstante Faktoren (z. B. die Betriebsform) nicht explizit berücksichtigt werden. Zweitens ist die Schätzung nicht effizient, wenn das Modell zeitlich persistente Elemente, wie z. B. den betrieblichen Flächeneinsatz, enthält. Im GMM-SYS Modelle werden die vorhandenen Informationen besser ausgenutzt, da neben der transformierten Gleichung (wie im GMM-OR) auch die untransformierte Gleichung für die Schätzung verwendet wird.

$$
\begin{aligned}
\ln L F_{i r f t}= & \alpha_{0}+\alpha_{1} \ln L F_{i r f, t-1}+\alpha_{2} \ln L F_{i r f, t-2}+\gamma_{1} \ln \text { Kap }_{i r f t}+ \\
& \gamma_{2} \ln A K_{i r f t}+\gamma_{3} \ln D Z_{i r f t}+\gamma_{4} \text { Gew }_{i r f t}+\gamma_{5} \text { AUM }_{i r f t}+ \\
& \gamma_{6} A Z_{i r f t}+\beta_{1} \text { Alter }_{i r f t}+\beta_{2} \ln \text { Pacht }_{r t}+\beta_{3} \text { Preis }_{r t}+ \\
& \beta_{4} \ln M L F_{r t}+\mu_{i}+\omega_{f}+\eta_{t}+v_{i r f t}, \quad t=2002, \ldots, 2005 .
\end{aligned}
$$

Zeitkonstante Effekte, wie die Betriebsform $\left(\omega_{f}\right)$, können im GMM-SYS berücksichtigt werden.

Aufgrund der dargestellten Überlegungen sollte die Qualität der Modellschätzung, im Sinne der unverzerrten Schätzung des Effektes der Programmteilnahme auf das betriebliche Flächenwachstum, von Modell POLS I bis zum Modell GMM-SYS zunehmen.

\subsection{Effekt der Programmteilnahme auf das betriebliche Flächen- wachstum}

Die deskriptive Statistik der verwendeten Stichprobe ist in Tabelle 9.1 dargestellt. Die Anzahl der Betriebe $(i=22.552)$ ist in jedem Modell gleich, da ein balanciertes Panel verwendet wird. Für die Analyse stehen sechs Untersuchungszeitpunkte $(T)$ und insgesamt 135.306 Beobachtungen zur Verfügung. Die Anzahl der verwendeten Untersuchungszeitpunkte und Beobachtungen variiert in Abhängigkeit von der Modellspezifikation.

Die Entwicklung der betrieblichen Flächenausstattung im Zeitraum 2000 bis 2005 in Betrieben mit und ohne Teilnahme an Agrarumweltmaßnahmen bzw. der Ausgleichszulage ist in Abbildung 9.1 dargestellt. Teilnehmerbetriebe (AUM, AZ) haben bereits im ersten Untersuchungsjahr (2000) im Mittel eine höhere Flächenausstattung als nicht teilnehmende Betriebe. AUM-Teilnehmer weisen im Untersuchungszeitraum ein höheres beobachtetes Flächenwachstum auf als die Gruppe der Nichtteilnehmer. In Betrieben mit und ohne Ausgleichszulage verläuft das betriebliche Flächenwachstum nahezu identisch. 
Inwieweit die beobachteten Unterschiede in der Flächenausstattung zwischen Teilnehmern und Nichtteilnehmern kausal auf die AUM- oder AZ-Teilnahme zurückzuführen sind, wird anhand der Schätzungen in Tabelle 9.2 überprüft. Welches dieser Modelle den höchsten Erklärungsgehalt hat und für die Ergebnisinterpretation genutzt wird, ist anhand der in Kapitel 7.2.2 (Modellselektion) diskutierten Kriterien entschieden.

Tabelle 9.1: $\quad$ Deskriptive Statistik der verwendeten Stichprobe

\begin{tabular}{|c|c|c|c|c|c|c|}
\hline Variable & Einheit & $\begin{array}{c}\text { Anzahl } \\
\text { Beobachtungen }\end{array}$ & Mittelwert & $\begin{array}{c}\text { Standard- } \\
\text { abweichung }\end{array}$ & Minimum & Maximum \\
\hline \multicolumn{7}{|l|}{ Betriebliche Merkmale } \\
\hline $\ln L F$ & ha & 135.306 & 3,992 & 0,590 & 2,307 & 7,474 \\
\hline Anteil Grünland & $\%$ & 135.306 & 29,051 & 27,955 & 0 & 100 \\
\hline AUM-Teilnahme & $0=$ nein, $1=\mathrm{ja}$ & 135.306 & 0,436 & 0,496 & 0 & 1 \\
\hline AZ-Teilnahme & $0=$ nein, $1=\mathrm{ja}$ & 135.306 & 0,359 & 0,480 & 0 & 1 \\
\hline$R G V / H F F$ (minmax) & $0=$ nein, $1=\mathrm{ja}$ & 135.306 & 0,116 & 0,320 & 0 & 1 \\
\hline ln $A U M$-Zahlungen & Euro & 135.306 & 3,015 & 3,426 & 0 & 6,908 \\
\hline $\ln A Z$-Zahlungen & Euro & 135.306 & 2,479 & 3,313 & 0 & 6,908 \\
\hline In Direktzahlungen & Euro & 135.306 & 0,878 & 0,238 & $-0,266$ & 1,331 \\
\hline $\ln$ Kapital & Euro & 135.306 & 13,316 & 0,732 & 4,456 & 17,629 \\
\hline $\ln A K$ Betrieb & Euro & 135.306 & 0,367 & 0,443 & $-4,605$ & 6,330 \\
\hline Gewinn & $100 \mathrm{~T}$ Euro & 135.306 & 0,127 & 0,500 & $-19,524$ & 62,608 \\
\hline Ausgaben Dünger (je ha) & TEuro & 135.306 & 0,102 & 0,049 & 0 & 1,588 \\
\hline Ausgaben PSM (je ha) & TEuro & 135.306 & 0,083 & 0,060 & 0 & 1,055 \\
\hline Alter Betriebsleiter & 10 Jahre & 135.306 & 4,880 & 1,035 & 0,400 & 9,100 \\
\hline$L V Z$ & Index & 135.306 & 18,185 & 6,801 & 0,001 & 66,586 \\
\hline Ackerbau & $0=$ nein, $1=\mathrm{ja}$ & 135.306 & 0,256 & 0,436 & 0 & 1 \\
\hline Futterbau & $0=$ nein, $1=\mathrm{ja}$ & 135.306 & 0,530 & 0,499 & 0 & 1 \\
\hline Veredlung & $0=$ nein, $1=\mathrm{ja}$ & 135.306 & 0,141 & 0,348 & 0 & 1 \\
\hline Gemischt & $0=$ nein, $1=\mathrm{ja}$ & 135.306 & 0,073 & 0,260 & 0 & 1 \\
\hline \multicolumn{7}{|l|}{ Regionale Merkmale } \\
\hline $\ln$ Pacht & 100 Euro/ha & 135.306 & 5,574 & 0,412 & 3,452 & 7,234 \\
\hline Preisindex & Jahr $2000=1 \%$ & 135.306 & 1,079 & 0,338 & 0,655 & 3,974 \\
\hline $\ln \varnothing L F /$ Betrieb & ha & 135.306 & 5,732 & 0,438 & 4,359 & 8,195 \\
\hline Schleswig-Holstein & $0=$ nein, $1=\mathrm{ja}$ & 135.306 & 0,002 & 0,044 & 0 & 1 \\
\hline Niedersachsen & $0=$ nein, $1=\mathrm{ja}$ & 135.306 & 0,218 & 0,413 & 0 & 1 \\
\hline Nordrhein-Westfalen & $0=$ nein, $1=\mathrm{ja}$ & 135.306 & 0,234 & 0,423 & 0 & 1 \\
\hline Hessen & $0=$ nein, $1=\mathrm{ja}$ & 135.306 & 0,021 & 0,145 & 0 & 1 \\
\hline Rheinland-Pfalz & $0=$ nein, $1=\mathrm{ja}$ & 135.306 & 0,049 & 0,217 & 0 & 1 \\
\hline \multicolumn{7}{|l|}{ Baden-Württemberg, } \\
\hline Saarland & $0=$ nein, $1=\mathrm{ja}$ & 135.306 & 0,188 & 0,391 & 0 & 1 \\
\hline Bayern & $0=$ nein, $1=\mathrm{ja}$ & 135.306 & 0,263 & 0,440 & 0 & 1 \\
\hline Brandenburg, Mecklenburg-Vorpommern & $0=$ nein, $1=\mathrm{ja}$ & 135.306 & 0,004 & 0,063 & 0 & 1 \\
\hline Sachsen-Anhalt & $0=$ nein, $1=\mathrm{ja}$ & 135.306 & 0,015 & 0,120 & 0 & 1 \\
\hline Thüringen & $0=$ nein, $1=\mathrm{ja}$ & 135.306 & 0,006 & 0,075 & 0 & 1 \\
\hline Dummy Betriebsform & $0=$ nein, $1=\mathrm{ja}$ & 135.306 & & siehe oben & 0 & 1 \\
\hline Dummy Land & $0=$ nein, $1=\mathrm{ja}$ & 135.306 & & siehe oben & 0 & 1 \\
\hline Dummy Kreis & $0=$ nein, $1=\mathrm{ja}$ & 135.306 & & nicht aufgeführt & 0 & 1 \\
\hline Dummy Jahr & $0=$ nein, $1=\mathrm{ja}$ & 135.306 & & nicht aufgeführt & 0 & 1 \\
\hline Anzahl Beobachtungen & $\mathrm{N}$ & 135.306 & & & & \\
\hline Anzahl Betriebe & $\mathrm{i}$ & 22.551 & & & & \\
\hline Anzahl Zeitpunkte $(2000, \ldots, 2005)$ & $\mathrm{T}$ & 6 & & & & \\
\hline
\end{tabular}

Regionale Merkmale beziehen sich auf die Merkmale von Kreisen und kreisfreien Städten (Nuts-III).

Quelle: eigene Berechnungen, Datenquellen vgl. Tabellen A2 und A3. 
Abbildung 9.1: $\quad$ Entwicklung der betrieblichen Flächenausstattung (2000 bis 2005) in Betrieben mit und ohne AUM- bzw. AZ-Teilnahme
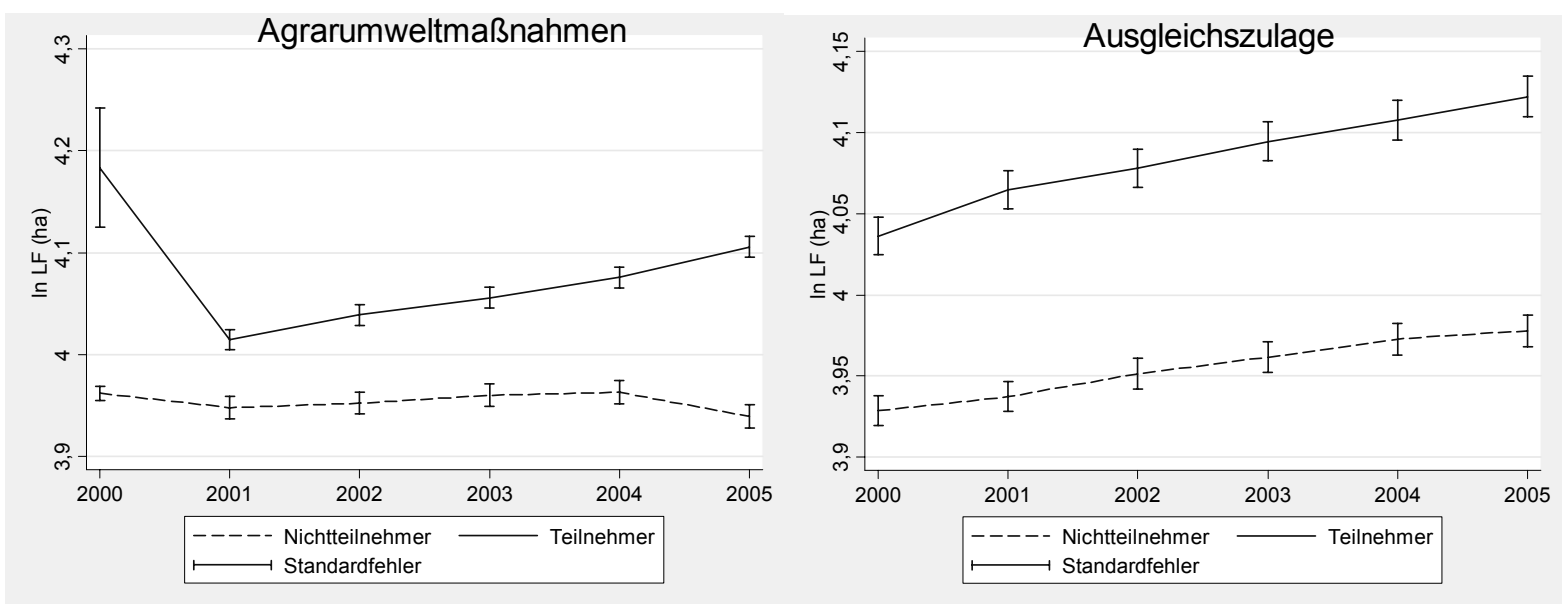

Die sprungartige Reduzierung der betrieblichen LF der AUM-Teilnehmer von 2000 bis 2001 ist auf den Beginn der AUM-Teilnahme von etwa der Hälfte der Betriebe zurückzuführen.

Quelle: eigene Berechnungen.

\section{Überprüfung der Modellannahmen, Vergleich der Modellgüte, Modellselektion ${ }^{63}$}

Im Folgenden werden die Annahmen der Modelle (Fehlerstruktur, Exogenität etc.) überprüft. Vergleichende Tests bzw. Modellgütemaße sind für die Modellselektion von untergeordneter Bedeutung, da die meisten Modelle bereits die Grundannahmen nicht erfüllen. Das zu bevorzugende Modell stützt sich stattdessen auf Überlegungen zur Richtung der möglichen Verzerrung der einzelnen Schätzer. Die Teststatistik ist dem unteren Teil der Tabelle $9.2 \mathrm{zu}$ entnehmen.

Die Nullhypothese der Abwesenheit von Autokorrelation erster (AR1) bzw. zweiter Ordnung (AR2) wurde für die Modelle POLS I, GMM-OR und GMM-SYS ${ }^{64}$ nicht abgelehnt (Arrellano-Bond-Test). Für alle Modelle werden gegenüber Autokorrelation und Heteroskedastizität robuste Varianzschätzer verwendet. 
Tabelle 9.2: $\quad$ Effekt der AUM- und AZ-Teilnahme auf das betriebliche Flächenwachstum

\begin{tabular}{|c|c|c|c|c|c|c|c|}
\hline $\begin{array}{l}\text { Abhängige Variable } \\
\text { Unabhängige Variablen }\end{array}$ & $\begin{array}{l}\ln L F \\
\text { Einheit }\end{array}$ & $\begin{array}{l}\text { POLS I } \\
\text { Koeff. p }\end{array}$ & $\begin{array}{l}\text { POLS II } \\
\text { Koeff. p }\end{array}$ & $\begin{array}{c}\text { FE I } \\
\text { Koeff. p }\end{array}$ & $\begin{array}{l}\text { FE II } \\
\text { Koeff. p }\end{array}$ & $\begin{array}{c}\text { GMM-OR } \\
\text { Koeff. p }\end{array}$ & $\begin{array}{r}\text { GMM-SYS } \\
\text { Koeff. p }\end{array}$ \\
\hline L. $\ln L F$ & ha & & $\begin{array}{l}0,960 \text { *** } \\
(0,005)\end{array}$ & & $\begin{array}{l}0,396 \text { *** } \\
(0,006)\end{array}$ & $\begin{array}{l}0,911 * * * \\
(0,142)\end{array}$ & $\begin{array}{l}0,969 \text { *** } \\
(0,122)\end{array}$ \\
\hline L2. & ha & & $\begin{array}{l}0,042 * * * \\
(0,005)\end{array}$ & & $\begin{array}{l}-0,016 * * * \\
(0,005)\end{array}$ & $\begin{array}{r}0,062 \\
(0,136)\end{array}$ & $\begin{array}{r}0,035 \\
(0,122)\end{array}$ \\
\hline $\ln$ Kapital & Euro & & & & & $\begin{array}{l}0,022 * * * \\
(0,008)\end{array}$ & $\begin{array}{l}0,013 \text { *** } \\
(0,003)\end{array}$ \\
\hline L. & Euro & $\begin{array}{l}0,212 * * * \\
(0,004)\end{array}$ & $\begin{array}{l}0,005 * * * \\
(0,0004)\end{array}$ & & $\begin{array}{l}0,006 * * * \\
(0,001)\end{array}$ & & \\
\hline $\ln A K$ Betrieb & $\mathrm{AKE}$ & & & & & $\begin{array}{r}0,029 \\
(0,018)\end{array}$ & $\begin{array}{l}0,008 * \\
(0,005)\end{array}$ \\
\hline L. & $\mathrm{AKE}$ & $\begin{array}{l}0,405 * * * \\
(0,010)\end{array}$ & $\begin{array}{l}0,007 * * * \\
(0,001)\end{array}$ & & $\begin{array}{l}0,009 * * * \\
(0,002)\end{array}$ & & \\
\hline In Direktzahlungen & Euro & & & & & $\begin{array}{l}-0,0002 \\
(0,002)\end{array}$ & $\begin{array}{r}0,001 \\
(0,002)\end{array}$ \\
\hline L. & Euro & $\begin{array}{l}0,066 * * * \\
(0,001)\end{array}$ & $\begin{array}{r}0,0002 \\
(0,0002)\end{array}$ & & $\begin{array}{l}0,00001 \\
(0,0002)\end{array}$ & & \\
\hline Gewinn & 100T Euro & & & & & $\begin{array}{l}-0,028 * * \\
(0,012)\end{array}$ & $\begin{array}{r}-0,007 \\
(0,010)\end{array}$ \\
\hline L. & 100T Euro & $\begin{array}{r}0,001 \\
(0,004)\end{array}$ & $\begin{array}{l}0,004 * * * \\
(0,001)\end{array}$ & & $\begin{array}{l}-0,002 * * * \\
(0,001)\end{array}$ & & \\
\hline Alter Betriebsleiter & 10 Jahre & $\begin{array}{l}-0,053 * * * \\
(0,003)\end{array}$ & $\begin{array}{l}-0,004 * * * \\
(0,0003)\end{array}$ & & & & $\begin{array}{l}-0,004 * * * \\
(0,001)\end{array}$ \\
\hline AUM-Teilnahme & $0=$ nein, $1=\mathrm{ja}$ & $\begin{array}{l}0,139 * * * \\
(0,006)\end{array}$ & $\begin{array}{l}0,003 * * * \\
(0,001)\end{array}$ & $\begin{array}{l}0,014 * * * \\
(0,001)\end{array}$ & $\begin{array}{l}0,005 * * * \\
(0,001)\end{array}$ & $\begin{array}{l}0,008 * * \\
(0,004)\end{array}$ & $\begin{array}{l}0,006 * * * \\
(0,002)\end{array}$ \\
\hline AZ-Teilnahme & $0=$ nein, $1=\mathrm{ja}$ & $\begin{array}{l}0,127 * * * \\
(0,008)\end{array}$ & $\begin{array}{l}0,002 * * \\
(0,001)\end{array}$ & $\begin{array}{l}0,010 * * * \\
(0,002)\end{array}$ & $\begin{array}{r}-0,001 \\
(0,002)\end{array}$ & $\begin{array}{r}0,002 \\
(0,006)\end{array}$ & $\begin{array}{r}0,001 \\
(0,003)\end{array}$ \\
\hline ln Pacht & 100 Euro/ha & $\begin{array}{r}0,006 \\
(0,005)\end{array}$ & $\begin{array}{r}-0,004 \\
(0,004)\end{array}$ & $\begin{array}{l}0,008 * * \\
(0,003)\end{array}$ & $\begin{array}{r}0,002 \\
(0,003)\end{array}$ & $\begin{array}{r}-0,004 \\
(0,004)\end{array}$ & $\begin{array}{r}-0,002 \\
(0,002)\end{array}$ \\
\hline Preisindex & $2.000=1 \%$ & $\begin{array}{l}-0,013 * * * \\
(0,001)\end{array}$ & $\begin{array}{l}-0,003 * * * \\
(0,001)\end{array}$ & $\begin{array}{l}-0,002 * * * \\
(0,001)\end{array}$ & $\begin{array}{l}-0,003 * * * \\
(0,001)\end{array}$ & $\begin{array}{l}-0,003 * * * \\
(0,001)\end{array}$ & $\begin{array}{l}-0,002 * * * \\
(0,001)\end{array}$ \\
\hline $\ln \varnothing L F /$ Betrieb & ha & $\begin{array}{l}0,229 * * * \\
(0,022)\end{array}$ & $\begin{array}{l}0,021 * * * \\
(0,011)\end{array}$ & $\begin{array}{l}0,100 * * * \\
(0,014)\end{array}$ & $\begin{array}{l}0,046 * * * \\
(0,010)\end{array}$ & $\begin{array}{l}0,025 * * \\
(0,011)\end{array}$ & $\begin{array}{r}-0,003 \\
(0,003)\end{array}$ \\
\hline Konstante & & $\begin{array}{l}-0,673 * * * \\
(0,142)\end{array}$ & $\begin{array}{l}-0,142 * * * \\
(0,065)\end{array}$ & $\begin{array}{l}3,350 * * * \\
(0,081)\end{array}$ & $\begin{array}{l}2,130 * * * \\
(0,069)\end{array}$ & & $\begin{array}{l}-0,144 * * * \\
(0,045)\end{array}$ \\
\hline $\begin{array}{l}\text { Dummy Betriebsform } \\
\text { Dummy Kreis }\end{array}$ & & $\begin{array}{l}\text { ja } \\
\text { ja }\end{array}$ & $\begin{array}{l}\text { ja } \\
\text { ja }\end{array}$ & & & & $\begin{array}{r}\text { ja } \\
\text { nein }\end{array}$ \\
\hline $\begin{array}{l}\text { Dummy Krels } \\
\text { Dummy Jahr }\end{array}$ & & $\begin{array}{l}\mathrm{ja} \\
\mathrm{ja}\end{array}$ & $\begin{array}{l}\mathrm{ja} \\
\mathrm{ja}\end{array}$ & ja & ja & ja & $\begin{array}{r}\text { nein } \\
\text { ja }\end{array}$ \\
\hline Beobachtungen & $N$ & 135.306 & 90.204 & 135.306 & 90.204 & 67.653 & 90.204 \\
\hline Betriebe & $i$ & 22.551 & 22.551 & 22.551 & 22.551 & 22.551 & 22.551 \\
\hline Zeitpunkte & $T$ & 6 & 4 & 6 & 4 & 3 & 4 \\
\hline $\begin{array}{l}\text { Instrumente } \\
\mathrm{R}^{2} \\
\mathrm{AIC}\end{array}$ & $n$ & $\begin{array}{r}0,57 \\
109.018\end{array}$ & $\begin{array}{r}0,98 \\
-211.493\end{array}$ & $\begin{array}{r}0,14 \\
-120.197\end{array}$ & $\begin{array}{r}0,98 \\
-268.696\end{array}$ & 20 & 32 \\
\hline $\begin{array}{l}\mathrm{F} \text { Test, Wald-Chi }{ }^{2} \text { Test } \\
\text { Hansen } J \text { Test }\end{array}$ & & $816^{* * *}$ & $218.397 * * *$ & $373 * * *$ & $519 * * *$ & $\begin{array}{l}3.635 * * * \\
4,73\end{array}$ & $\begin{array}{c}1.030 .000 * * * \\
11,37\end{array}$ \\
\hline AR(1) Test & & $78,64 * * *$ & $-0,77$ & $-4,79 * * *$ & $-2,82 * * *$ & $-3.57 * * *$ & $-4,12 * * *$ \\
\hline $\operatorname{AR}(2)$ Test & & $76,40 * * *$ & 0,96 & $6,60 * * *$ & $-1,86 *$ & $-0,03$ & 0,18 \\
\hline
\end{tabular}

1. L. $=$ Wert des Vorjahres $(t-1)$. L2. $=$ Wert für $t-2$.

2. Gegenüber Heteroskedastizität und Autokorrelation robuste Standardfehler in Klammern.

3. Signifikanzniveau: $* * * \mathrm{p}<0,01, * * \mathrm{p}<0,05, * \mathrm{p}<0,1$.

4. $\mathrm{H}_{0}$ Hansen $J$ Test: Verwendete Instrumente sind gemeinsam valide.

5. $\mathrm{H}_{0} \mathrm{AR}(1)$-Test, AR(2)-Test: Keine Autokorrelation erster und zweiter Ordnung.

6. Zweistufige GMM-Schätzung, Korrektur der Standardfehler nach WINDMEIJER (2000), kollabierte Instrumentenmatrix.

7. Verwendete Instrumente in GMM-OR und GMM-SYS (transformierte Gleichung): $\ln L_{t-3 / 4}, \ln K a p_{t-2 / 3}, \ln D Z_{t-2 / 3}, \ln A K_{t-2 / 3}$, ln Gew $_{t-2 / 3}, A U M_{t-1 / 2}, A Z_{t-1 / 2}, \ln$ Pacht $_{t}$, Preis $_{t}, \ln M L F_{t}, \mu_{t}$, nur GMM-SYS: Alter $t, \omega_{t}$

8. Verwendete Instrumente in GMM-SYS (untransformierte Gleichung): $\ln L F_{t-2}, \ln K_{a p} p_{t-2}, \ln D Z_{t-2}, \ln A K_{t-2}, \ln G e w_{t-2}, A U M_{t-1}, A Z_{t-1}$, Alter $_{t}, \ln$ Pacht $_{t}$, Preis $_{t}, \ln M L F_{t}, \mu_{t}$

Quelle: eigene Berechnungen, Datenquellen vgl. Tabellen A2 und A3. 
Die Annahme der Konstanz der Schätzer wird für alle Modelle abgelehnt (Chow-Test). Eine Verletzung dieser Standardannahme wird zunächst akzeptiert, da die Modellschätzung für die gesamtdeutsche Stichprobe durchgeführt wird. Inwieweit der Effekt der Programmteilnahme in Abhängigkeit von betrieblichen Merkmalen variiert, wird in Kapitel 9.3 untersucht. Die gemeinsame und singuläre Validität der in den GMM-Modellen verwendeten Instrumente (Hansen $J$ Test, Differenz-in-Hansen Statistik) wurde in keinem Fall auf einem signifikanten Niveau abgelehnt. Die als endogen identifizierten Regressoren werden im POLS II und FE II als Lags berücksichtigt, wenngleich hierdurch die Endogenität nicht vollständig beseitigt werden kann.

Die Annahme der Stationarität des zu untersuchenden Prozesses gilt für die dynamischen Modelle (FE II, POLS II, GMM-Modelle). Die Annahme konnte nicht direkt überprüft werden, da entsprechende Einheitswurzeltests (HADRI, 2000; KWIATOWSKI et al., 1992) nur für Daten mit mehr als 30 Beobachtungszeitpunkten geeignet sind. Die für Panelmodelle formulierte Bedingung $\left|\alpha_{1}+\alpha_{2}\right|<1$ ist nur im FE II und GMM-OR erfüllt. Insgesamt erscheint jedoch die Anzahl der Untersuchungszeitpunkte zu gering, um belastbare Aussagen hinsichtlich der Stationaritiät der Daten zu machen. Ein Datentransformation sollte ausreichen, um gegebenenfalls nicht-stationäre Daten in stationäre zu überführen. Der Hausman-Test bestätigt, dass die Verwendung von FE-Modellen (statt Random-EffektModellen) gerechtfertigt ist, da der betriebspezifische Effekt $\left(\eta_{i}\right)$ mit einem oder mehreren Regressoren korreliert.

Die globale Nullhypothese wird in allen Modellen auf einem Signifikanzniveau von unter $1 \%$ abgelehnt. Durch die Modelle POLS II und FE II werden jeweils 98 \% der Modellvarianz erklärt. Anhand des LR-Tests (nicht dargestellt) und des AIC lässt sich jedoch ein höherer Erklärungsgehalt des FE II Modells festmachen. Die Varianzaufklärung der statischen Modelle (POLS I, FE I) ist dagegen deutlich geringer. Dies legt nahe, dass die Bestimmungsgründe des betrieblichen Flächenwachstums durch ein dynamisches Modell besser abgebildet werden als durch ein statisches Modell.

Im Gegensatz zum POLS II und FE II erfüllen die GMM-Modelle die zentralen Modellannahmen (keine Autokorrelation, Exogenität von Regressoren bzw. Instrumenten). Die Schätzer der GMM-Modelle sind daher vermutlich am wenigsten verzerrt. ${ }^{65}$ Welches der beiden GMM-Modelle zu bevorzugen ist, kann unter Zuhilfenahme der Ergebnisse des POLS II und FE II entschieden werden. Der Koeffizient für den ersten Lag der abhängigen Variable ist im POLS II nach oben verzerrt (da der betriebsspezifische Effekt nicht kontrolliert wird) und im FE II nach unten verzerrt (da within transformierte Lags endogener

65 Das für die GMM-Schätzungen verwendete Programm (xtabond2) unterstützt nicht den Log Likelihood basierten AIC. Das Gütemaß kann daher nicht zum Vergleich aller Modelle herangezogen werden. 
bzw. vordeterminierter Regressoren verwendet wurden). Ein weniger verzerrter Schätzer sollte demnach im Bereich zwischen den Koeffizienten des POLS II $(0,396)$ und FE II $(0,960)$ liegen (BOND, 2002: 5). Der Koeffizient des GMM-OR liegt mit 0,911 innerhalb dieses Bereiches, der Koeffizient des GMM-SYS knapp darüber. Die Ergebnisinterpretation erfolgt daher auf Basis des GMM-OR.

\section{Interpretation der Ergebnisse}

Im oberen Teil der Tabelle 9.2 sind die geschätzten Koeffizienten mit robusten Standardfehlern dargestellt. Die Koeffizienten logarithmierter Regressoren sind als Elastizitäten zu interpretieren: Erhöht sich z. B. der betriebliche Kapitaleinsatz um ein Prozent, so führt dies zu einem betrieblichen Flächenwachstum in Höhe von 2,2 \% (Modell GMM-OR). Die Koeffizienten nicht logarithmierter Regressoren spiegeln die prozentuale Zunahme der betrieblichen LF wider, wenn sich der Wert des Regressors um eine Einheit ändert.

Der Effekt der AUM-Teilnahme bzw. der AZ-Teilnahme entspricht dem prozentualen Unterschied des jährlichen Flächenwachstums mit und ohne Programmteilnahme. Betriebe mit AUM-Teilnahme weisen im Durchschnitt ein um 0,8 \% höheres Flächenwachstum pro Jahr auf als Betriebe ohne Teilnahme. Dieser Effekt ist im GMM-OR (GMM-SYS) auf einem Niveau von unter $5 \%$ (unter $1 \%$ ) signifikant.

Der Effekt der AUM- und AZ-Teilnahme auf die betriebliche Flächenentwicklung, kontrolliert für die in den spezifizierten Modellen enthalten Faktoren, ist in Abbildung 9.2 graphisch dargestellt.

Abbildung 9.2: $\quad$ Mittelwerte der geschätzten (logarithmierten) Flächenentwicklung in Betrieben mit und ohne AUM- und AZ-Teilnahme
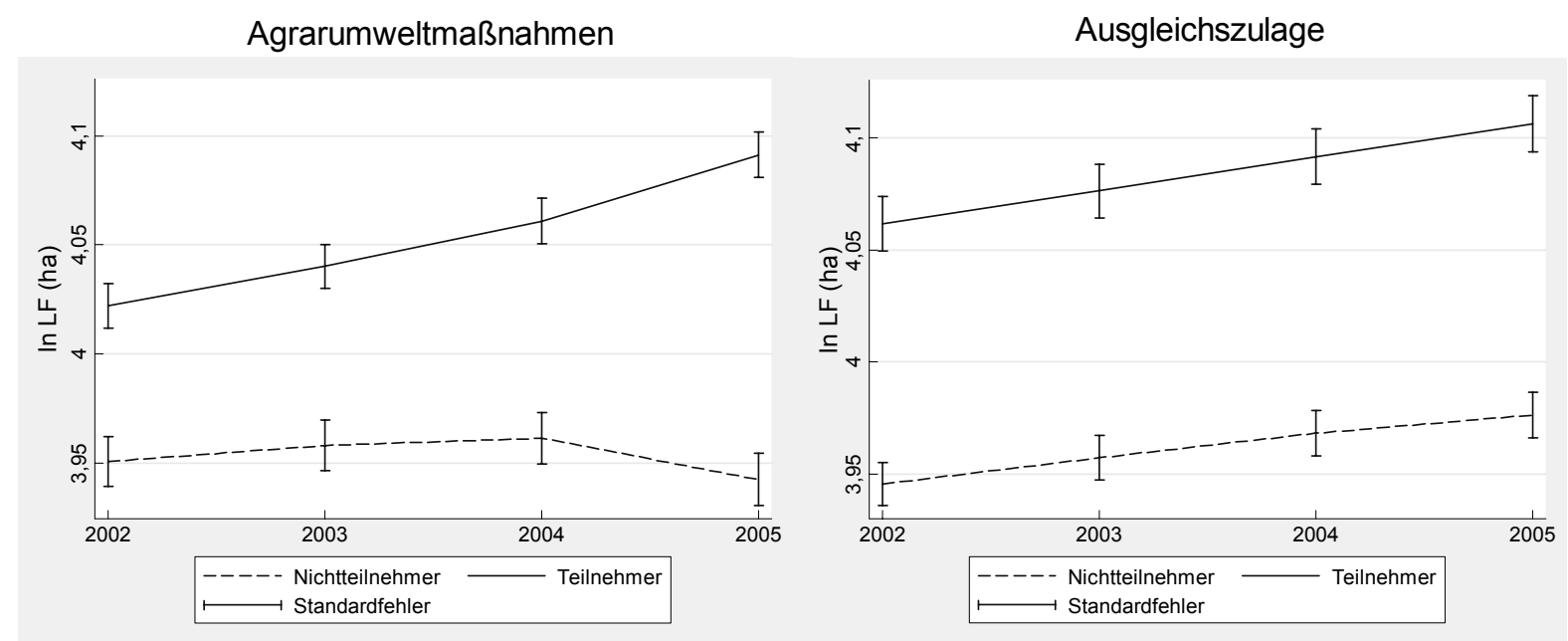

Quelle: eigene Schätzung auf Basis des Modells GMM-OR. 
$\mathrm{Zu}$ erkennen ist, dass

- Teilnehmer bereits vor der Programmteilnahme eine höhere Flächenausstattung aufweisen als Nichtteilnehmer,

- AUM-Teilnehmer im Mittel deutlich höhere Wachstumsraten haben als Nichtteilnehmer und

- die Flächenentwicklung in Betrieben mit und ohne Ausgleichszulage nahezu identisch verläuft.

Der positive Effekt der AUM-Teilnahme auf das betriebliche Flächenwachstum bestätigt die Ergebnisse der Matching-Analyse (vgl. Tabelle 8.2). Das höhere Flächenwachstum der Teilnehmerbetriebe ist auf die Flächenbindung der AUM-Zahlungen zurückzuführen. Durch die AUM-Zahlungen verringern sich die Grenzkosten des eingesetzten Bodens. Die Nutzung von Boden wird, im Vergleich zu anderen Faktoren, günstiger.

Der geschätzte Effekt der AUM-Teilnahme im Modell GMM-OR unterscheidet sich nur marginal von den entsprechenden Koeffizienten in den Modellen GMM-SYS, POLS II und FE II. In den statischen Modellen (POLS I, FE I) wird der Effekt der AUM-Teilnahme deutlich überschätzt, da der Einfluss der LF der Vorjahre bzw. unbeobachtete, betriebsspezifische Effekte nicht kontrolliert werden. In Analogie gilt dies auch für den Effekt der AZ-Teilnahme, der mit zunehmender Kontrolle unbeobachteter Effekte abnimmt.

Um die Größenordnung des Effektes der AUM- bzw. AZ-Teilnahme auf das Flächenwachstum einzuordnen, wird der geschätzte Effekt der Programmteilnahme auf die absolute, nicht logarithmierte Flächenausstattung der Teilnehmerbetriebe bezogen. Tabelle 9.3 weist die geschätzte LF der Teilnehmer, die geschätzte (potenzielle) LF der Teilnehmer in der Referenzsituation ohne das Programm sowie die Differenz zwischen beiden Parametern aus. Die Differenz entspricht dem Effekt der Programmteilnahme auf die Teilnehmer $(A T T)$.

Die geschätzte Flächenausstattung von AUM-Teilnehmern beträgt im Durchschnitt der Untersuchungsjahre 69,79 ha. Die potenzielle Flächenausstattung eines Teilnehmers in der Referenzsituation ohne AUM-Teilnahme wäre 69,26 ha gewesen. Die Differenz zwischen beiden Parametern, 0,53 ha, entspricht dem mittleren jährlichen Effekt der AUMTeilnahme auf die Teilnehmer und ist auf einem Niveau von unter $5 \%$ signifikant. Bezogen auf eine 5-jährige AUM-Teilnahme entspricht dies einem mittleren Flächenzuwachs von etwa 2,5 ha je Teilnehmerbetrieb im untersuchten Zeitraum.

Der Effekt der AZ-Teilnahme auf die Flächenausstattung beträgt im Durchschnitt 0,16 ha pro Jahr, ist jedoch nicht statistisch signifikant. 
Tabelle 9.3: Mittlerer jährlicher Effekt der AUM- und AZ-Teilnahme auf die betriebliche Flächenentwicklung

\begin{tabular}{|c|c|c|c|c|c|c|c|}
\hline & Einheit & POLS I & POLS II & FE I & FE II & GMM-OR & GMM-SYS \\
\hline \multicolumn{8}{|l|}{ Agrarumweltmaßnahmen } \\
\hline (1) LF Teilnehmer & ha & 67,71 & 69,01 & 54,70 & 57,37 & 69,79 & 69,14 \\
\hline $\begin{array}{l}\text { (2) Potentielle LF der Teilnehmer } \\
\text { bei Nichtteilnahme }\end{array}$ & ha & 58,94 & 68,81 & 53,94 & 57,11 & 69,26 & 68,72 \\
\hline $\mathrm{ATT}=(1)-(2)$ & ha & $8,77^{* * *}$ & $0,20 * * *$ & $0,76 * * *$ & $0,26 * * *$ & $0,53 * *$ & $0,42 * * *$ \\
\hline \multicolumn{8}{|l|}{ Ausgleichszulage } \\
\hline (1) LF Teilnehmer & ha & 70,35 & 70,95 & 54,16 & 57,80 & 71,20 & 70,94 \\
\hline $\begin{array}{l}\text { (2) Potentielle LF der Teilnehmer } \\
\text { bei Nichtteilnahme }\end{array}$ & ha & 61,99 & 70,81 & 53,60 & 57,85 & 71,05 & 70,85 \\
\hline $\mathrm{ATT}=(1)-(2)$ & ha & $8,36 * * *$ & $0,13 * *$ & $0,56 * * *$ & $-0,05$ & 0,16 & 0,09 \\
\hline
\end{tabular}

1. Die Nullhypothese $\left(\mathrm{ATT}_{\mathrm{AUM}}=0, \mathrm{ATT}_{\mathrm{AZ}}=0\right)$, wurde durch den Wald Test, angewendet auf die lineare Vorausschätzung $(\mathrm{xb})$, überprüft. Signifikanzniveau: $* * * \mathrm{p}<0,01, * * \mathrm{p}<0,05, * \mathrm{p}<0,1$.

2. Die dargestellte LF der Betriebe entspricht der exponenzierten Vorausschätzung, berechnet als $\left(\exp (\ln L F)+\exp \left(0,5 \sigma^{2}\right)\right.$. Methode vgl. CAMERON und TRIVEDI (2005: 105).

Quelle: eigene Berechnungen, Datenquellen vgl. Tabellen A2 und A3.

Die Angaben in Tabelle 9.3 beziehen sich auf die untersuchte Stichprobe. Im Vergleich zur Grundgesamtheit sind in der Stichprobe Betriebe mit einer hohen Flächenausstattung überrepräsentiert (vgl. Tabelle 6.2). Der Programmeffekt (ATT) in Tabelle 9.3 dürfte daher den wahren Effekt der Programmteilnahme an Agrarumweltmaßnahmen und an der Ausgleichszulage in Deutschland eher überschätzen.

Die übrigen Koeffizienten des GMM-OR haben überwiegend das erwartete Vorzeichen und Niveau (vgl. Tabelle 9.2). Die betriebliche Flächenausstattung des Vorjahres (L. $\ln L F$ ) hat unter allen berücksichtigten Regressoren den höchsten Erklärungsgehalt $(91,1 \%)$ für die gegenwärtige Flächenausstattung eines Betriebes und ist auf einem Niveau von unter $1 \%$ signifikant. Dies untermauert nochmals die Angemessenheit der dynamischen Modellspezifikation bei der Untersuchung des betrieblichen Flächenwachstums.

Eine Erhöhung des Kapitaleinsatzes (ln Kapital) hat einen positiven Einfluss auf die betriebliche Flächenausstattung. Ein Anstieg des Kapitaleinsatzes um $1 \%$ Prozent führt zu einer Zunahme der LF um 2,2\%. Der Effekt ist auf einem Niveau von unter $1 \%$ signifikant. Der Zusammenhang zwischen dem Arbeitseinsatz (ln Arbeit) und dem Bodeneinsatz ist nicht signifikant von Null verschieden. 
Der Effekt von gekoppelten Direktzahlungen auf das betriebliche Flächenwachstum ist in allen Modellen, mit Ausnahme des POLS I, nicht signifikant. Für gekoppelte Direktzahlungen ist theoretisch ein positiver Effekt auf die betriebliche Flächenausstattung zu erwarten, da die Höhe der Direktzahlungen mit zunehmender Fläche bzw. zunehmendem Tierbesatz steigt. Dieser Zusammenhang ist durch zahlreiche empirische Studien belegt (AHEARN et al., 2002; GoOdWIN et al., 2007; KEY und RoBERTs, 2003).

Der um Direktzahlungen, Agrarumweltzahlungen und die Ausgleichszulage bereinigte Gewinn hat im GMM-OR einen signifikant negativen Einfluss auf das betriebliche Wachstum (-2,8 \%). In den übrigen Modellen (POLS II, FE II, GMM-SYS) variiert der Koeffizient des Gewinns auf einem deutlich niedrigen Niveau, ist aber wie im GMM-OR tendenziell negativ. Ein niedriger Gewinn führt zu einer relativ niedrigen Entlohnung der eingesetzten Produktionsfaktoren.

Das Alter des Betriebsleiters (Alter Betriebsleiter) hat im Modell GMM-SYS einen signifikant negativen Einfluss auf das betriebliche Flächenwachstum. Eine Zunahme des Alters um zehn Jahre verringert das betriebliche Flächenwachstum um durchschnittlich 0,4 \% pro Jahr. Das Niveau dieses Effektes ist in allen Modellen relativ konstant und auf einem Niveau von unter $1 \%$ signifikant. Die hohen Wachstumsraten von Betrieben mit jungen Betriebsleitern stehen in einem direktem Zusammenhang mit der Investitionstätigkeit: Eine hohe Investitionstätigkeit in Betrieben mit relativ jungen Betriebsleitern (unter 40 Jahren) ist durch den Agrarbericht belegt (BMVEL, 2005). Mit zunehmendem Alter gehen die Investitionstätigkeit und das betriebliche Wachstum zurück.

WeISS (1999) und GaLE (1994) zeigen, dass der Zusammenhang zwischen dem betrieblichen Wachstum und dem Alter des Betriebsleiters durch einen nichtlinearen Lebenszykluseffekt geprägt ist: Das betriebliche Wachstum nimmt mit zunehmenden Alter zunächst zu (positiver Zusammenhang), ab einem Alter von etwa 36 bis 45 Jahren wieder ab (negativer Zusammenhang) und in einem hohen Alter des Betriebsleiters wieder zu (positiver Zusammenhang). Ein identisches Muster wäre auch bei einer Berücksichtigung quadrierter und kubischer Terme des Alters des Betriebsleiters in den spezifizierten Modellen zu beobachten gewesen. ${ }^{66}$ Den zunächst positiven Zusammenhang zwischen Alter und dem betrieblichen Wachstum führt JovANOVIC (1982) auf die mit der Zeit zunehmenden Managementfähigkeiten jüngerer Betriebsleiter zurück. Das stagnierende bzw. negative Wachstum von Betrieben mit älteren Betriebsleitern wird mit dem abnehmenden Zeithorizont, in dem der Nutzen des betrieblichen Wachstums realisiert werden kann, und mit der gesundheitlich bedingten Reduzierung der Arbeitsbelastung begründet.

Quadrierte und kubische Terme des Alters des Betriebsleiters werden in den spezifizierten Modellen nicht berücksichtigt, da diese nur einen unbedeutenden zusätzlichen Erklärungsgehalt haben. 
Gegen Ende der Erwerbsphase des Betriebsleiters nimmt das betriebliche Wachstum wieder zu, vermutlich in Vorbereitung der anstehenden Hofübergabe.

Die landwirtschaftlichen Erzeugerpreise (Preisindex) haben einen negativen Einfluss auf die betriebliche Flächenentwicklung. Dieser Effekt ist in allen Modellen auf einem Niveau von unter $1 \%$ signifikant. Ein Rückgang des Preisindex um $10 \%$ Prozentpunkte gegenüber dem Referenzjahr 2000 (100\%) verringert die betriebliche LF um durchschnittlich 0,3\%. SCHMitT und ANDERMANN (1996) führen diesen Zusammenhang auf die Überwälzung der Agrarpreise auf die Boden- bzw. Pachtpreise zurück. Sinkende Erzeugerpreise verringern die Entlohnung der eingesetzten Faktoren. In Volkswirtschaften mit einer geringen wirtschaftlichen Bedeutung des Agrarsektors führt dies primär zur Verringerung der Entlohnung des Faktors Boden.

Die durchschnittliche Größe landwirtschaftlicher Betriebe in einem Kreis (ln Ø $L F /$ Betrieb) wurde berücksichtigt, um den Einfluss der regionalen Agrarstruktur auf die betriebliche Flächenentwicklung zu kontrollieren. Eine hohe durchschnittliche Betriebsgröße im Kreis scheint mit einem hohen einzelbetrieblichen Wachstum zu korrespondieren. Die höchsten betrieblichen Wachstumsraten sind in den Kreisen zu beobachten, in denen vergleichsweise große Betriebe angesiedelt sind. MARGARIAN (2007) zeigte in einer aggregierten Analyse für Deutschland (1979 bis 2003), dass die Anzahl ausscheidender Betriebe in Kreisen mit einer großbetrieblichen Struktur signifikant höher ist als in allen anderen Kreisen. Aufgrund der stärkeren Flächenfreisetzung durch aussteigende Betriebe kann in diesen Kreisen mit einer großbetrieblichen Struktur das betriebliche Wachstum in vergleichsweise größeren Schritten erfolgen.

\subsection{Heterogenität der Effekte der Programmteilnahme}

Die Ergebnisse in Tabelle 9.2 beruhen auf der Annahme, dass der Effekt von Agrarumweltmaßnahmen und der Ausgleichszulage für alle Betriebe gleich ist. Bereits die Ergebnisse der Matching-Analyse zeigen, dass die Effekte der Programmteilnahme in Abhängigkeit von regionalen und betrieblichen Merkmalen variieren (Kapitel 8.4).

Für die Analyse heterogener Effekte der Programmteilnahme werden die Modelle POLS II, FE II, GMM-OR und GMM-SYS - erweitert um Interaktionsterme bzw. modifiziert zu einem Selektionsmodell - verwendet. Die Modellspezifikation bleibt gegenüber den Modellen in Tabelle 9.2 unverändert. Die Ergebnispräsentation beschränkt sich daher auf den interessierenden Effekt der AUM- und AZ-Teilnahme in Abhängigkeit von betrieblichen Merkmalen (Höhe der Programmzahlungen, Anteil der LF in benachteiligten Gebieten, Betriebsgröße, Betriebsform, Teilnahmewahrscheinlichkeit, Alter des Betriebsleiters) und regionalen Merkmalen (Bundesland). 


\subsubsection{Effekt der Programmteilnahme in Abhängigkeit von der Höhe der Programmzahlungen}

Aus den verwendeten Buchführungsdaten ist nicht ersichtlich, wie viel Hektar der betrieblichen LF tatsächlich unter den Auflagen von Agrarumweltmaßnahmen bzw. der Ausgleichszulage bewirtschaftet werden. Als Proxy für den flächenmäßigen Umfang der Programmteilnahme wird daher die Höhe der Programmzahlungen verwendet. Zu erwarten ist, dass der Effekt der Programmteilnahme mit zunehmender Höhe der Programmzahlungen ansteigt. Tabelle 9.4 zeigt die Effekte der Programmteilnahme in Abhängigkeit von der Höhe der Programmzahlungen je Betrieb.

Im Modelltyp A wird die Summe der vom Betrieb erhaltenen AUM- bzw. AZ-Zahlungen als erklärende Variable verwendet. Die $A U M$ - bzw. AZ-Zahlungen betragen für alle Nichtteilnehmer den Wert Null Euro; für Teilnehmer nehmen sie einen Wert größer Null an. Die linksseitig gestauchte Skalierung der Programmzahlungen wird in den Selektionsmodellen (Heckman AUM, Heckman AZ) explizit berücksichtigt; der Einfluss unbeobachteter, betriebsspezifischer Faktoren wird jedoch nicht kontrolliert, da lediglich gepoolte Daten verwendet werden.

Tabelle 9.4: $\quad$ Effekt der AUM- und AZ-Teilnahme auf das betriebliche Flächenwachstum in Abhängigkeit von der Höhe der Programmzahlungen

\begin{tabular}{|c|c|c|c|c|c|c|c|c|c|c|}
\hline \multirow{2}{*}{$\begin{array}{l}\text { Abhängige Variable } \\
\text { Unabhängige Variablen }\end{array}$} & \multirow{2}{*}{$\begin{array}{l}\ln L F \\
\text { Einheit }\end{array}$} & \multirow{2}{*}{$\begin{array}{l}\text { Heckman } \\
\text { AUM } \\
\text { Koeff. p }\end{array}$} & \multirow{2}{*}{$\begin{array}{l}\text { Heckman } \\
\qquad \mathbf{A Z} \\
\text { Koeff. p }^{1)}\end{array}$} & \multicolumn{2}{|l|}{ POLS II } & \multicolumn{2}{|l|}{ FE II } & \multicolumn{2}{|l|}{ GMM-OR } & \multirow{2}{*}{$\begin{array}{l}\text { GMM-SYS } \\
\text { Koeff. } p^{1)} p^{2)}\end{array}$} \\
\hline & & & & Koeff. $p^{1)}$ & $\mathrm{p}^{2)}$ & Koeff. $p^{1)}$ & $\mathrm{p}^{2)}$ & Koeff. $p^{1)}$ & $\mathrm{p}^{2)}$ & \\
\hline \multicolumn{11}{|l|}{ Modelltyp A } \\
\hline $\ln A U M$-Zahlungen & Euro & $\begin{array}{r}0,002 * * * \\
(0,0004)\end{array}$ & & $\begin{array}{l}0,0005 * * * \\
(0,0001)\end{array}$ & & $\begin{array}{l}0,001 * * * \\
(0,0002)\end{array}$ & & $\begin{array}{l}0,002 * * \\
(0,001)\end{array}$ & & $\begin{array}{l}0,0004 * * * \\
(0,0001)\end{array}$ \\
\hline ln AZ-Zahlungen & Euro & & $\begin{array}{r}0,001 \\
(0,001)\end{array}$ & $\begin{array}{c}0,0004 * * * \\
(0,0001)\end{array}$ & & $\begin{array}{r}0,0001 \\
(0,0003)\end{array}$ & & $\begin{array}{r}0,001 \\
(0,001)\end{array}$ & & $\begin{array}{r}-0,0001 \\
(0,0003)\end{array}$ \\
\hline \multicolumn{11}{|l|}{ Modelltyp B } \\
\hline \multicolumn{11}{|c|}{ AUM-Teilnehmer mit Zahlungen in Höhe von ... } \\
\hline O bis 1.000 Euro & $0=$ nein, $\mathrm{ja}=1$ & & & 0,001 & & $0,004 * * *$ & & $0,008 * * *$ & & $-0,003$ \\
\hline 1.000 bis 3.000 Euro & $0=$ nein, $\mathrm{ja}=1$ & & & $0,003 * * *$ & & $0,004 * *$ & & 0,005 & & 0,001 \\
\hline 3.000 bis 5.000 Euro & $0=$ nein, $\mathrm{ja}=1$ & & & $0,005 * * * *$ & $* * *$ & $0,007 * * *$ & & 0,008 & & 0,002 \\
\hline $\begin{array}{l}\text { über } 5.000 \text { Euro } \\
\text { 3) }\end{array}$ & $0=$ nein, $\mathrm{ja}=1$ & & & $0,006 * * * *$ & $\begin{array}{l}* * * \\
* * *\end{array}$ & $0,009 * * *$ & * & 0,008 & & 0,00005 \\
\hline \multicolumn{11}{|c|}{ AZ-Teilnehmer mit Zahlungen in Höhe von ... } \\
\hline 0 bis 1.000 Euro & $0=$ nein, $\mathrm{ja}=1$ & & & $-0,0002$ & & $-0,001$ & & $-0,001$ & & $-0,002$ \\
\hline 1.000 bis 3.000 Euro & $0=$ nein, $\mathrm{ja}=1$ & & & $0,002 *$ & & $-0,004 *$ & & $-0,005$ & & 0,003 \\
\hline 3.000 bis 5.000 Euro & $0=$ nein, $\mathrm{ja}=1$ & & & $0,004 * * *$ & $* * *$ & 0,002 & & 0,015 & & 0,007 \\
\hline $\begin{array}{l}\text { über } 5.000 \text { Euro } \\
\text { 3) }\end{array}$ & $0=$ nein, $\mathrm{ja}=1$ & & & $0,006 * * *$ & $\begin{array}{l}* * * \\
* * *\end{array}$ & $0,011 * * *$ & $\begin{array}{l}* * * \\
* * *\end{array}$ & 0,012 & $* * *$ & 0,006 \\
\hline
\end{tabular}

1. Signifikanzniveau: $* * * \mathrm{p}<0,01, * * \mathrm{p}<0,05, * \mathrm{p}<0,1$.

2. Nullhypothesen des Wald Tests: ${ }^{1)}$ Effekt der Teilnahme ist Null. ${ }^{2)}$ Effekt ist im Vergleich zu Teilnehmern mit 0 bis 1.000 Euro gleich. ${ }^{3)}$ Effekt der Teilnahme ist für alle Teilnehmer gleich.

Quelle: eigene Berechnungen, Datenquellen vgl. Tabellen A2 und A3. 
Eine Zunahme der AUM-Zahlungen um ein Prozent führt im Durchschnitt zu einer Erhöhung der betrieblichen Flächenausstattung um 0,2\% (Heckman-Modell AUM). Dieser Effekt ist auf einem Niveau von unter $1 \%$ signifikant und konsistent mit den Ergebnissen in Tabelle 9.2. Der Effekt der AZ-Zahlungen auf das Flächenwachstum ist statistisch nicht signifikant (Heckman-Modell AZ). Die Effekte der Programmzahlungen variieren zwischen den verschiedenen Modellen nur marginal. Dies legt nahe, dass der mit der Programmteilnahme verbundene Selektionseffekt durch die Heckman-Modelle nicht systematisch besser oder schlechter kontrolliert wird.

Interessant ist auch hier ein Blick auf den Einfluss von gekoppelten Direktzahlungen auf die betriebliche Flächenentwicklung: Das POLS I ausgenommen, haben gekoppelte Direktzahlungen einen signifikanten Einfluss auf die betriebliche Flächenausstattung (vgl. ausführliche Ergebnisdarstellung in Tabelle A6). Es ist zu vermuten, dass die AUMTeilnahme bzw. der Erhalt von AUM-Zahlungen die betriebliche Flächenentwicklung vergleichsweise stärker beeinflusst, als die in Summe wesentlich höheren Direktzahlungen. Ein möglicher Erklärungsansatz hierfür ist, dass Direktzahlungen die Entlohnung des Bodens in allen Betrieben gleichmäßig verbessern, da die meisten Betriebe Direktzahlungen erhalten. Von der durch AUM-Zahlungen verbesserten Faktorentlohnung profitieren dagegen nur teilnehmende Betriebe. Die AUM-Teilnehmer sind in der Lage, höhere Pachtpreise zu zahlen als die nicht an Agrarumweltmaßnahmen teilnehmenden Nachbarbetriebe.

Im Modelltyp B (Tabelle 9.4) wird der Einfluss von Klassen unterschiedlich hoher AUMbzw. AZ-Zahlungen auf das Flächenwachstum untersucht. Unterschieden werden geringe (0 bis 1.000 Euro), niedrige (1000 bis 3.000 Euro), mittlere (3.000 bis 5.000 Euro) und hohe (über 5.000 Euro) Programmzahlungen.

Der Einfluss der AUM-Zahlungen auf die betriebliche Flächenausstattung nimmt mit steigender Höhe der Programmzahlungen zu. Je nach Modell variiert dieser Effekt zwischen Null (GMM-SYS) bzw. 0,8\% (GMM-OR) in Betrieben mit AUM-Zahlungen unter 1.000 Euro und zwischen Null (GMM-Modelle) und 0,9\% (FE-Modell) in Betrieben mit über 5.000 Euro AUM-Zahlungen pro Jahr. Im FE II Modell ist der Einfluss der AUMZahlungen in jedem Fall signifikant - und zwar sowohl im Vergleich zu nicht teilnehmenden Betrieben als auch im Vergleich zwischen teilnehmenden Betrieben mit unterschiedlich hohen Programmzahlungen.

Auch die Ergebnisse für die Ausgleichszulage lassen einen positiven Zusammenhang zwischen der Höhe der Programmzahlungen und dem betrieblichen Flächenwachstum vermuten. Der Effekt der AZ-Teilnahme liegt in Betrieben mit Programmzahlungen über 5.000 Euro pro Jahr zwischen 1,1 \% (FE-Modell) und Null (GMM-Modelle). Allerdings erhalten in den Alten Bundesländern vergleichsweise wenige Betriebe AZ-Zahlungen in dieser Höhe (vgl. Tabelle 2.2), so dass die praktische Relevanz dieses Ergebnisses gering 
sein dürfte. Betriebe mit AZ-Zahlungen unter 5.000 Euro weisen tendenziell ein geringeres betriebliches Flächenwachstum auf, wenngleich die entsprechenden Koeffizienten häufig nicht statistisch signifikant sind.

\subsubsection{Effekt der Programmteilnahme in Abhängigkeit vom Anteil der betrieblichen $\mathrm{LF}$ in benachteiligten Gebieten}

Für die Betriebe mit Ausgleichszulage ist bekannt, welcher Anteil der betrieblichen LF in der benachteiligten Gebietskulisse liegt $(0 \%$, 0 bis $50 \%$, 50 bis $100 \%$, $100 \%)$. In Deutschland werden nur etwa $50 \%$ der LF in benachteiligten Gebieten tatsächlich gefördert (GAK-Berichterstattung, div. Jgg.). Aus diesem Grund ist der Anteil der betrieblichen LF in benachteiligten Gebieten vermutlich ein schlechter Proxy für den flächenmäBigen Teilnahmeumfang eines Betriebes an der Ausgleichszulage.

Die Ergebnisse in Tabelle A7 (GMM-OR) zeigen, dass der Effekt der AZ-Teilnahme (AUM-Teilnahme) auf das betriebliche Flächenwachstum nur für die Betriebe signifikant ist, deren Flächen zu $100 \%$ in benachteiligten Gebieten liegen. Diese Betriebe weisen ein um 1,9 \% (1,5\%) höheres Flächenwachstum auf als Nichtteilnehmer, deren LF ebenfalls vollständig im benachteiligten Gebiet liegt. Dieser Effekt ist auf einem Niveau von unter $10 \%(5 \%)$ signifikant.

Je höher die im benachteiligen Gebiet bewirtschaftete LF ist, desto höher sind die Effekte der Ausgleichszulage und von Agrarumweltmaßnahmen bezogen auf das betriebliche Flächenwachstum.

\subsubsection{Effekte der Programmteilnahme nach Bundesländern}

Agrarumweltmaßnahmen und die Ausgleichszulage werden in Deutschland auf Ebene der Bundesländer umgesetzt. Unterschiede zwischen den Bundesländern bestehen z. B. hinsichtlich der Förderbedingungen, der gewährten Prämienhöhen, dem Spektrum angebotener Maßnahmen und der Anzahl der geförderten Betriebe (vgl. Kapitel 2.2.2 und 2.3.2). Des Weiteren unterscheiden sich die Bundesländer hinsichtlich ihrer Produktions-, Verarbeitungs- und Vermarktungsstruktur des primären Sektors. Daher ist zu vermuten, dass sich der Effekt der Programmteilnahme zwischen den Bundesländern unterscheidet.

Tabelle 9.5 zeigt den Effekt der AUM- und AZ-Teilnahme auf das betriebliche Flächenwachstum differenziert nach Bundesländern. Aus den Ergebnissen in Tabelle 9.5 geht hervor, dass der Effekt der AUM-Teilnahme auf das Flächenwachstum in Bayern $(+0,7$ \%) in fast allen Modellen signifikant positiv ist. Einen tendenziell negativen Effekt der Pro- 
grammteilnahme auf das Flächenwachstum verzeichnen AUM-Teilnehmer in SchleswigHolstein und AZ-Teilnehmer in Thüringen. Da der Effekt der AUM-Teilnahme in Schleswig-Holstein deutlich negativ ist, unterscheidet sich dieser signifikant vom Effekt der AUM-Teilnahme in anderen Bundesländern (GMM-SYS, Hypothese 2).

Insgesamt variiert der Effekt der AUM- und AZ-Teilnahme auf das betriebliche Flächenwachstum zwischen den Bundesländern geringfügig. Diese Unterschiede sind allerdings nur selten statistisch signifikant bzw. folgen keinem systematischen Muster.

Tabelle 9.5: Effekt der AUM- und AZ-Teilnahme auf das betriebliche Flächenwachstum nach Bundesländern

\begin{tabular}{|c|c|c|c|c|c|c|c|}
\hline Abhängige Variable & $\ln L F$ & POLS II & & FE II & GMM-OR & GMM-SY & \\
\hline Unabhängige Variablen & Einheit & Koeff. $p^{1)}$ & $\mathrm{p}^{2)}$ & Koeff. $p^{1)} p^{2)}$ & Koeff. $p^{1)} p^{2)}$ & Koeff. $p^{1)}$ & $\mathrm{p}^{2)}$ \\
\hline \multicolumn{8}{|l|}{ AUM-Teilnehmer in . } \\
\hline Schleswig-Holstein & $0=$ nein, $\mathrm{ja}=1$ & $-0,035 *$ & & $-0,020$ & $-0,029$ & $-0,040 * * *$ & \\
\hline Niedersachsen & $0=$ nein,$j a=1$ & $0,004 * * *$ & & 0,002 & 0,006 & 0,004 & $* * *$ \\
\hline Nordrhein-Westfalen & $0=$ nein, $j a=1$ & 0,002 & & 0,003 & 0,004 & 0,003 & $* * *$ \\
\hline Hessen & $0=$ nein,$j a=1$ & 0,003 & & $0,011 * *$ & 0,027 & 0,005 & $* * *$ \\
\hline Rheinland-Pfalz & $0=$ nein, $j a=1$ & $-0,002$ & & $-0,007$ & $-0,019$ & 0,001 & $* * *$ \\
\hline Baden-Württemberg, Saarland & $0=$ nein, $j a=1$ & $0,008 * *$ & & 0,004 & 0,010 & 0,006 & $* * *$ \\
\hline Bayern & $0=$ nein, $\mathrm{ja}=1$ & $0,004 * * *$ & & $0,007 * * *$ & 0,010 & $0,007 * *$ & $* * *$ \\
\hline Brandenburg, Mecklenburg-Vorpommern & $0=$ nein,$j a=1$ & $-0,004$ & & $-0,002$ & 0,017 & 0,019 & \\
\hline Sachsen-Anhalt & $0=$ nein, $\mathrm{ja}=1$ & 0,003 & & $0,013 *$ & $-0,001$ & 0,006 & $* * *$ \\
\hline $\begin{array}{l}\text { Thüringen } \\
\text { 3) }\end{array}$ & $0=$ nein, $j a=1$ & 0,010 & & 0,021 & $-0,038$ & 0,008 & $* * *$ \\
\hline \multicolumn{8}{|l|}{ AZ-Teilnehmer in ... } \\
\hline Schleswig-Holstein & $0=$ nein,$j a=1$ & \multicolumn{6}{|c|}{ keine Teilnehmer } \\
\hline Niedersachsen & $0=$ nein, $\mathrm{ja}=1$ & 0,002 & & $-0,007$ & 0,019 & 0,006 & \\
\hline Nordrhein-Westfalen & $0=$ nein, $\mathrm{ja}=1$ & 0,002 & & $-0,005$ & 0,015 & 0,003 & \\
\hline Hessen & $0=$ nein, $\mathrm{ja}=1$ & $0,008 * *$ & & 0,011 & 0,008 & 0,008 & \\
\hline Rheinland-Pfalz & $0=$ nein, $\mathrm{ja}=1$ & 0,001 & & $-0,006$ & 0,006 & $-0,010$ & \\
\hline Baden-Württemberg, Saarland & $0=$ nein, $\mathrm{ja}=1$ & 0,001 & & 0,002 & $-0,001$ & 0,001 & \\
\hline Bayern & $0=$ nein, $\mathrm{ja}=1$ & $0,004 * * *$ & & $-0,003$ & $-0,012$ & $-0,005$ & \\
\hline Brandenburg, Mecklenburg-Vorpommern & $0=$ nein, $\mathrm{ja}=1$ & 0,014 & & 0,045 & 0,005 & 0,025 & \\
\hline Sachsen-Anhalt & $0=$ nein, $\mathrm{ja}=1$ & $-0,008$ & & 0,012 & 0,056 & 0,031 & \\
\hline $\begin{array}{l}\text { Thüringen } \\
\text { 3) }\end{array}$ & $0=$ nein, $\mathrm{ja}=1$ & $-0,019 * *$ & $* *$ & $-0,021$ & $-0,056^{*}$ & $-0,027$ & \\
\hline
\end{tabular}

1. Signifikanzniveau: *** $\mathrm{p}<0,01, * * \mathrm{p}<0,05, * \mathrm{p}<0,1$.

2. Nullhypothesen des Wald Tests: ${ }^{1)}$ Effekt der Teilnahme ist Null. ${ }^{2)}$ Effekt ist im Vergleich zu Teilnehmern in Schleswig-Holstein (AUM) bzw. Niedersachsen (AZ) gleich. ${ }^{3)}$ Effekt der Teilnahme ist für alle Teilnehmer gleich.

3. Die Ausgleichszulage wurde in Niedersachsen im Jahr 1999 eingestellt. AZ-Zahlungen von in Niedersachsen ansässigen Betrieben beziehen sich vermutlich auf Flächen in anderen Bundesländern oder sind auf Fehlbuchungen zurückzuführen.

Quelle: eigene Berechnungen, Datenquellen vgl. Tabellen A2 und A3. 


\subsubsection{Effekt der Programmteilnahme nach Betriebsformen}

Zwischen den Betriebsformen variierende Effekte sind vorrangig für Agrarumweltmaßnahmen zu erwarten: Futterbaubetriebe nehmen vor allem an grünlandbezogenen Extensivierungsmaßnahmen teil. Um die für die AUM-Teilnahme maximal zulässige Viehbesatzdichte zu erreichen, wird in der Regel Fläche zugepachtet. Eine Zunahme der betrieblichen LF ist zu erwarten. Ackerbaulich orientierte Agrarumweltmaßnahmen (Mulch- und Direktsaatverfahren, umweltfreundliche Gülleausbringung) werden überwiegend von Marktfruchtbetrieben oder flächenstarken Futterbaubetrieben in Anspruch genommen. Von diesen produktionstechnisch ausgerichteten Agrarumweltmaßnahmen ist kein Effekt auf die betriebliche Flächenausstattung zu erwarten.

Tabelle 9.6 zeigt den Effekt der AUM- und AZ-Teilnahme auf die betriebliche Flächenentwicklung differenziert nach Betriebsformen. Einen signifikant positiven Effekt der AUM-Teilnahme verzeichnen - je nach Modell - Ackerbau- und Futterbaubetriebe. Die Effekte der Teilnahme an der Ausgleichszulage unterscheiden sich zwischen den einzelnen Betriebsformen nicht signifikant.

Tabelle 9.6: Effekt der AUM- und AZ-Teilnahme auf die betriebliche Flächenentwicklung, differenziert nach Betriebformen

\begin{tabular}{|c|c|c|c|c|c|c|}
\hline Abhängige Variable & $\ln L F$ & POLS II & & FE II & GMM-OR & GMM-SYS \\
\hline Unabhängige Variablen & Einheit & Koeff. $p^{1)}$ & $p^{2)}$ & Koeff. $p^{1)} p^{2)}$ & Koeff. $p^{1)} p^{2)}$ & Koeff. $p^{1)} p^{2}$ \\
\hline \multicolumn{7}{|c|}{ AUM-Teilnehmer der Betriebsform } \\
\hline Ackerbau & $0=$ nein, $j a=1$ & $0,005 * * *$ & & $0,008 * * *$ & $-0,001$ & $0,008 * * *$ \\
\hline Futterbau & $0=$ nein, $\mathrm{ja}=1$ & $0,002 * * *$ & & $0,003 * *$ & $0,011 * *$ & 0,004 \\
\hline Veredlung & $0=$ nein, $j a=1$ & 0,002 & & 0,004 & $-0,002$ & 0,000 \\
\hline $\begin{array}{l}\text { Gemischt } \\
\text { 3) }\end{array}$ & $0=$ nein, $\mathrm{ja}=1$ & $-0,0001$ & & $-0,002$ & 0,005 & 0,002 \\
\hline \multicolumn{7}{|c|}{ AZ-Teilnehmer der Betriebsform } \\
\hline Ackerbau & $0=$ nein, $j a=1$ & 0,001 & & $-0,0004$ & $-0,001$ & $-0,003$ \\
\hline Futterbau & $0=$ nein, $j a=1$ & $0,003 * * *$ & & 0,001 & $-0,001$ & 0,003 \\
\hline Veredlung & $0=$ nein, $\mathrm{ja}=1$ & $-0,002$ & & $-0,006$ & $-0,010$ & $-0,005$ \\
\hline Gemischt & $0=$ nein, $\mathrm{ja}=1$ & 0,004 & ** & $-0,004$ & 0,005 & 0,001 \\
\hline
\end{tabular}

1. Signifikanzniveau: *** $\mathrm{p}<0,01, * * \mathrm{p}<0,05, * \mathrm{p}<0,1$.

2. Nullhypothesen des Wald Tests: ${ }^{1)}$ Effekt der Teilnahme ist Null. ${ }^{2)}$ Effekt ist im Vergleich zu Teilnehmern Ackerbau gleich. ${ }^{3}$ ) Effekt der Teilnahme ist für alle Teilnehmer gleich.

Quelle: eigene Berechnungen, Datenquellen vgl. Tabellen A2 und A3.

AUM-Futterbaubetriebe verzeichnen ein um $0,2 \%$ bis $1,1 \%$ höheres Flächenwachstum pro Jahr als Futterbaubetriebe ohne AUM-Teilnahme (Flächenaufstockung zur Reduzierung der Viehbesatzdichte). Ein Ackerbaubetrieb mit AUM-Teilnahme hat im Durchschnitt und pro Jahr ein um $0,8 \%$ höheres Flächenwachstum als ein nicht teilnehmender 
Ackerbaubetrieb. Der Effekt ist mit einer Irrtumswahrscheinlichkeit von unter $1 \%$ signifikant (FE II, GMM-SYS). Die Beobachtung ist zunächst überraschend, da die Teilnahme an ackerbaulich ausgerichteten Agrarumweltmaßnahmen keine Ausweitung der LF, z. B. infolge einer Flächenaufstockung im Rahmen der Grünlandextensivierung, erfordert.

Die Teilnahmeraten von Ackerbaubetrieben an Agrarumweltmaßnahmen sind mit der Einführung der Modulationsmaßnahmen im Jahr 2003 sprunghaft angestiegen. Es ist also zu vermuten, dass der wachstumsfördernde Effekt in AUM-Ackerbaubetrieben vor allem auf die im Jahr 2003 eingeführten Modulationsmaßnahmen zurückzuführen ist. Aufschluss darüber gibt eine zeitlich differenzierte Analyse des Effektes der AUM-Teilnahme für die Zeit vor und nach 2003 (vgl. Tabelle A11).

Tabelle A11 im Anhang zeigt, dass der Effekt der AUM-Teilnahme in Ackerbau- und Futterbaubetrieben im Zeitraum vor Einführung der Modulationsmaßnahmen (2000 bis 2003) in fast allen Modellen insignifikant sind. In den Jahren nach der Einführung der Modulationsmaßnahmen (2004 bis 2005) erhöht sich der Effekt für Ackerbaubetriebe auf $1 \%$ (FEModell) und für Futterbaubetriebe auf 1,1\% bis 1,6 \% (GMM-Modelle). Das Ergebnis ist ein Indiz dafür, dass die Modulationsmaßnahmen einen positiveren Einfluss auf das betriebliche Flächenwachstum haben als die vor 2003 implementierten Agrarumweltmaßnahmen. $\mathrm{Zu}$ vermuten ist, dass die Modulationsmaßnahmen weniger die Kosten für die Anwendung umweltfreundlicher Produktionsmethoden kompensieren als vielmehr eine Verbilligung des Produktionsfaktors Boden darstellen.

\subsubsection{Effekt der Programmteilnahme nach Betriebsgröße}

Tabelle 9.7 zeigt den Effekt der AUM- und AZ-Teilnahme auf das betriebliche Flächenwachstum differenziert nach Betrieben unterschiedlicher Größenklassen. Im Gegensatz zu den zuvor präsentierten Schätzungen weichen die Ergebnisse der GMM-Modelle deutlich von denen des POLS II und FE II ab. Die Ergebnisinterpretation stützt sich auf die als eher konsistent anzusehenden GMM-Schätzungen.

Der Zusammenhang zwischen der Betriebsgröße in der Ausgangssituation und dem (relativen) betrieblichen Wachstum - bekannt als das Gesetz von Gibrat - ist Ausgangspunkt zahlreiche empirischer Untersuchungen. Das Gesetz von Gibrat postuliert die Unabhängigkeit der Betriebsgröße vom betrieblichen Wachstum, da das betriebliche Wachstum vorrangig auf zufällige Faktoren zurückzuführen ist (HALLAM, 1987). Untersuchungen von UPTON und HAWORTH (1987) und Evans (1987) bestätigen diesen Zusammenhang. WeISS (1999) und SHAPIRO et al. (1987) kommen zu dem gegenteiligen Ergebnis, dass kleine Betriebe im Durchschnitt ein höheres Wachstum aufweisen als große Betriebe. WEISs be- 
gründet diese Beobachtung damit, dass kleine Betriebe entweder aussteigen oder schnell bis zu einem Punkt mit einer minimal effizienten Betriebsgröße wachsen.

Tabelle 9.7: Effekt der AUM- und AZ-Teilnahme auf das betriebliche Flächenwachstum in Abhängigkeit von der Betriebsgröße

\begin{tabular}{|c|c|c|c|c|c|c|c|}
\hline Abhängige Variable & $\ln L F$ & POLS II & & FE II & GMM-O & & GMM-SYS \\
\hline Unabhängige Variablen & Einheit & Koeff. $p^{1)}$ & $p^{2)}$ & Koeff. $p^{1)} p^{2)}$ & Koeff. $p^{1)}$ & $\mathrm{p}^{2)}$ & Koeff. $p^{1)} p^{2)}$ \\
\hline \multicolumn{8}{|c|}{ AUM-Teilnehmer mit Betriebsgröße ... } \\
\hline unter 20 ha LF & $0=$ nein, $j a=1$ & 0,003 & & 0,008 & 0,025 & & $0,033 *$ \\
\hline 20 bis 50 ha LF & $0=$ nein, $\mathrm{ja}=1$ & $0,002 * * *$ & & $0,005 * * *$ & $0,013 * *$ & & $0,012 * * *$ \\
\hline 50 bis $100 \mathrm{ha} \mathrm{LF}$ & $0=$ nein, $\mathrm{ja}=1$ & $0,003 * * *$ & & 0,002 & $-0,002$ & & 0,002 \\
\hline über 100 ha LF & $0=$ nein, $\mathrm{ja}=1$ & $0,005 * * *$ & & $0,010 * * *$ & 0,004 & & 0,007 \\
\hline 3) & & & & & & $* *$ & \\
\hline \multicolumn{8}{|c|}{ AZ-Teilnehmer mit Betriebsgröße ... } \\
\hline unter 20 ha LF & $0=$ nein, $\mathrm{ja}=1$ & $-0,006 *$ & & $-0,010$ & 0,016 & & 0,006 \\
\hline 20 bis 50 ha LF & $0=$ nein, $\mathrm{ja}=1$ & $0,002 *$ & * & $-0,002$ & $-0,005$ & & 0,0001 \\
\hline 50 bis $100 \mathrm{ha} \mathrm{LF}$ & $0=$ nein, $j a=1$ & $0,003 * *$ & * & 0,001 & $-0,009$ & & 0,00002 \\
\hline$\underset{3)}{\text { über } 100 \text { ha LF }}$ & $0=$ nein,$j a=1$ & 0,001 & & $-0,001$ & $-0,001$ & & 0,007 \\
\hline
\end{tabular}

1. Signifikanzniveau: $* * * \mathrm{p}<0,01, * * \mathrm{p}<0,05, * \mathrm{p}<0,1$.

2. Nullhypothesen des Wald Tests: ${ }^{1)}$ Effekt der Teilnahme ist Null. ${ }^{2)}$ Effekt ist im Vergleich zu Teilnehmern kleiner 20 ha LF gleich. ${ }^{3)}$ Effekt der Teilnahme ist für alle Teilnehmer gleich.

Quelle: eigene Berechnungen, Datenquellen vgl. Tabelle A2 und A3.

Die Ergebnisse in Tabelle 9.7 (GMM-OR) zeigen, dass der Effekt der AUM-Teilnahme auf das Flächenwachstum nicht in allen Betriebsgrößenklassen gleich ist. Die entsprechende Nullhypothese (Hypothese 3) wurde im Modell GMM-OR auf einem Niveau von unter $5 \%$ abgelehnt. Der wachstumsfördernde Effekt der AUM-Teilnahme ist lediglich in kleinen Betrieben (unter 20 ha LF, 20 bis 50 ha LF) statistisch signifikant. In großen Betrieben (50 bis 100 ha LF, über 100 ha LF) ist der Effekt der AUM-Teilnahme nicht signifikant von Null verschieden.

Kleine Betriebe scheinen demnach stärker vom wachstumsfördernden Effekt der AUMTeilnahme zu profitieren als große Betriebe. Dieser Zusammenhang lässt sich jedoch nicht statistisch absichern. Ein paarweiser Vergleich von kleinen und großen AUM-Teilnehmern zeigt keine statistisch signifikanten Unterschiede hinsichtlich der Wachstumsraten (Hypothese 2). Die Hypothese, dass der Effekt von Agrarumweltmaßnahmen auf das Flächenwachstum systematisch durch die Betriebsgröße beeinflusst wird, ist daher abzulehnen. Die Ergebnisse in Tabelle 9.7 sind nicht uneingeschränkt als Bestätigung des Gesetzes von Gibrat zu interpretieren, da hier nicht der singuläre Effekt der Betriebsgröße, sondern der aggregierte Effekt der Betriebsgröße und der Programmteilnahme auf das betriebliche Wachstum gemessen wird. 
Der Effekt der AZ-Teilnahme auf das betriebliche Flächenwachstum erreicht - außer im POLS II - kein statistisch signifikantes Niveau und variiert dementsprechend auch nicht signifikant zwischen AZ-Teilnehmern unterschiedlicher Betriebsgröße.

\subsubsection{Effekt der Programmteilnahme in Abhängigkeit von der Teil- nahmewahrscheinlichkeit}

Tabelle 9.8 zeigt den Einfluss der Wahrscheinlichkeit der AUM- und AZ-Teilnahme auf das betriebliche Flächenwachstum. Die GMM-Schätzungen sind vermutlich aufgrund der hohen Anzahl an Dummyvariablen und Interaktionstermen inkonsistent und daher nicht dargestellt.

Tabelle 9.8: Effekt der AUM- und AZ-Teilnahme auf das betriebliche Flächenwachstum in Abhängigkeit von der Wahrscheinlichkeit der Programmteilnahme

\begin{tabular}{|c|c|c|c|c|c|c|c|}
\hline Abhängige Variable & $\ln L F$ & POLS II & & FE II & & GMM-OR & GMM-SYS \\
\hline Unabhängige Variablen & Einheit & Koeff. $\mathrm{p}^{1)}$ & $\mathrm{p}^{2)}$ & Koeff. $p^{1)}$ & $\mathrm{p}^{2)}$ & Koeff. $\mathrm{p}^{1)} \mathrm{p}^{2)}$ & Koeff. $\mathrm{p}^{1)} \mathrm{p}^{2)}$ \\
\hline \multicolumn{8}{|c|}{ AUM-Teilnehmer mit Wahrscheinlichkeit der Teilnahme ... } \\
\hline $0 \%$ bis $20 \%$ & $0=$ nein,$j a=1$ & $0,003 *$ & & 0,002 & & & \\
\hline $20 \%$ bis $40 \%$ & $0=$ nein,$j a=1$ & $0,003 * *$ & & $0,003 *$ & & & \\
\hline $40 \%$ bis $60 \%$ & $0=$ nein,$j a=1$ & $0,006 * * *$ & & 0,003 & & & \\
\hline $60 \%$ bis $80 \%$ & $0=$ nein, $\mathrm{ja}=1$ & $0,003 * *$ & & $0,010 * * *$ & & & \\
\hline $\begin{array}{l}80 \% \text { bis } 100 \% \\
\text { 3) }\end{array}$ & $0=$ nein, $\mathrm{ja}=1$ & $0,006 * * *$ & & 0,003 & $*$ & $\begin{array}{c}\text { Koeffizienten } \\
\text { inkonsistent }\end{array}$ & $\begin{array}{c}\text { Koeffizienten } \\
\text { inkonsistent }\end{array}$ \\
\hline \multicolumn{8}{|c|}{ AZ-Teilnehmer mit Wahrscheinlichkeit der Teilnahme ... } \\
\hline $0 \%$ bis $20 \%$ & $0=$ nein,$j a=1$ & 0,001 & & $-0,001$ & & & \\
\hline $20 \%$ bis $40 \%$ & $0=$ nein,$j a=1$ & $-0,002$ & & $-0,003$ & & & \\
\hline $40 \%$ bis $60 \%$ & $0=$ nein,$j a=1$ & $-0,004 * * *$ & & $-0,008$ & & & \\
\hline $60 \%$ bis $80 \%$ & $0=$ nein, $\mathrm{ja}=1$ & $-0,003 *$ & & $-0,005 *$ & & & \\
\hline $80 \%$ bis $100 \%$ & $0=$ nein, $\mathrm{ja}=1$ & 0,002 & & 0,005 & & 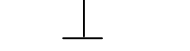 & 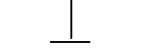 \\
\hline
\end{tabular}

1. Signifikanzniveau: *** $\mathrm{p}<0,01, * * \mathrm{p}<0,05, * \mathrm{p}<0,1$.

2. Nullhypothesen des Wald Tests: ${ }^{1)}$ Effekt der Teilnahme ist Null. ${ }^{2)}$ Effekt ist im Vergleich zu Teilnehmern mit 0 bis $20 \%$ gleich. ${ }^{3)}$ Effekt der Teilnahme ist für alle Teilnehmer gleich.

Quelle: eigene Berechnungen, Datenquellen vgl. Tabelle A2 und A3.

Die Wahrscheinlichkeit der AUM- und AZ-Teilnahme wurde je Programm und Jahr ${ }^{67}$ in separaten Probit-Modellen geschätzt und entspricht dem in der Matching-Analyse verwendeten Propensity Score. Die Ergebnisse der Probit-Schätzungen sind in den Tabellen A8 und A9 dargestellt. Der LR-Test zeigt, dass die Signifikanz der geschätzten Modelle $1 \%$

67 Durch die Schätzung von $t$ Probit-Modellen - anstelle eines Probit-Modells mit $t$ Zeitpunkten werden autokorrelierte Fehler vermieden (WOOLDRIDGE, 1995). 
oder besser ist. Die Vorhersagegüte der Modelle ist mit $35 \%$ (AUM) bzw. 54 \% (AZ) Varianzaufklärung gut.

Tabelle 9.8 zeigt einen positiven Zusammenhang zwischen der Teilnahmewahrscheinlichkeit eines Betriebes und dem Niveau des Programmeffektes auf das Flächenwachstum. Der Effekt der Programmteilnahme ist desto größer, je höher die Wahrscheinlichkeit eines Betriebes ist, an Agrarumweltmaßnahmen bzw. an der Ausgleichszulage teilzunehmen. So verzeichnen AUM-Teilnehmer mit einer Teilnahmewahrscheinlichkeit von 20 bis $40 \%$ ein positives Flächenwachstum in Höhe von 0,3 \% pro Jahr und AUM-Teilnehmer der Wahrscheinlichkeitsklasse 60 bis $80 \%$ ein in etwa drei mal so hohes Flächenwachstum $(+1,0 \%)$. Für Teilnehmer an der Ausgleichszulage ist der Zusammenhang weniger ausgeprägt, da die Effekte der Ausgleichszulage insgesamt geringer sind.

Aus Tabelle 9.8 ergibt sich die Frage, ob die meisten Teilnehmer eine niedrige oder eine hohe Teilnahmewahrscheinlichkeit aufweisen. Abbildung A7 im Anhang zeigt, dass über die Hälfte der analysierten AUM-Teilnehmer eine Teilnahmewahrscheinlichkeit von über $80 \%$ haben. Für diese Betriebe ist also ein vergleichsweise hoher Effekt der Programmteilnahme auf das betriebliche Flächenwachstum zu beobachten.

\subsubsection{Effekt der Programmteilnahme nach Alter des Betriebsleiters}

Eine vielfach geäußerte Vermutung ist, dass Agrarumweltmaßnahmen von älteren Betriebsleitern als „Überbrückung bis zur Rente“ genutzt werden. Ein einfacher Chi-QuadratTest bestätigt, dass ältere Betriebsleiter signifikant häufiger an Agrarumweltmaßnahmen teilnehmen als jüngere Betriebsleiter (vgl. Tabelle A10).

Die überproportionale Teilnahme von älteren Betriebsleitern an Agrarumweltmaßnahmen dürfte auf verschiedene Gründe zurückzuführen sein. Befragungsergebnisse aus Nordrhein-Westfalen, Hessen und Niedersachsen (REITER et al., 2003) zeigen, dass mit der Teilnahme an Agrarumweltmaßnahmen teilweise intensive Betriebszweige (z. B. die Milchproduktion) aufgegeben werden. Eine Motivation zu diesem Schritt kann die Reduzierung der Arbeitsbelastung aus gesundheitlichen Gründen sein. Denkbar ist auch, dass auf notwendige betriebliche Investitionen, mit Blick auf das nahende Rentenalter, verzichtet wird.

Die Ergebnisse in Tabelle 9.9 zeigen, dass der Effekt der AUM-Teilnahme auf das Flächenwachstum mit zunehmendem Alter des Betriebsleiters steigt. Der Effekt ist für Betriebsleiter über 60 Jahre am höchsten (von $+0,6 \%$ bis 1,0\%) und signifikant von Null verschieden. Der Effekt der AZ-Teilnahme ist ebenfalls für Betriebe mit Betriebsleitern über 60 Jahre am größten, jedoch nur im POLS II signifikant. 
Tabelle 9.9: Effekte der AUM- und AZ-Teilnahme nach Alter des Betriebsleiters

\begin{tabular}{|c|c|c|c|c|c|c|c|c|}
\hline Abhängige Variable & $\ln L F$ & POLS II & & FE II & & GMM-OR & & GMM-SYS \\
\hline Unabhängige Variable & Einheit & Koeff $p^{1)}$ & $\mathrm{p}^{2)}$ & Koeff $\mathrm{p}^{1)}$ & $\mathrm{p}^{2)}$ & Koeff $p^{1)}$ & $\mathrm{p}^{2)}$ & Koeff $p^{1)} p^{2)}$ \\
\hline \multicolumn{9}{|c|}{ AUM-Teilnehmer nach Altersklassen } \\
\hline unter 45 Jahre & $0=$ nein, $j a=1$ & 0,001 & & 0,002 & & 0,006 & & 0,004 \\
\hline 45 bis 60 Jahre & $0=$ nein, $\mathrm{ja}=1$ & $0,003 * * *$ & & $0,004 * *$ & & 0,002 & & $0,007 * * *$ \\
\hline über 60 Jahre & $0=$ nein, $\mathrm{ja}=1$ & $0,006 * * *$ & $* * *$ & $0,010 * * *$ & $* * *$ & $0,028 * * *$ & * & $0,007 * *$ \\
\hline 3) & & & $* * *$ & & ** & & ** & \\
\hline \multicolumn{9}{|c|}{ AZ-Teilnehmer nach Altersklassen } \\
\hline unter 45 Jahre & $0=$ nein, $j a=1$ & $0,002 *$ & & $-0,003$ & & $-0,002$ & & $-0,004$ \\
\hline 45 bis 60 Jahre & $0=$ nein, $\mathrm{ja}=1$ & 0,001 & & $-0,001$ & & 0,001 & & 0,004 \\
\hline $\begin{array}{l}\text { über } 60 \text { Jahre } \\
3 \text { 3) }\end{array}$ & $0=$ nein, $\mathrm{ja}=1$ & $0,003 * *$ & & 0,003 & & 0,008 & & 0,003 \\
\hline
\end{tabular}

1. Signifikanzniveau: *** $\mathrm{p}<0,01, * * \mathrm{p}<0,05, * \mathrm{p}<0,1$.

2. Nullhypothesen des Wald Tests: ${ }^{1)}$ Effekt der Teilnahme ist Null. ${ }^{2)}$ Effekt ist im Vergleich zu Teilnehmern unter 45 Jahre gleich. ${ }^{3)}$ Effekt der Teilnahme ist für alle Teilnehmer gleich.

Quelle: eigene Berechnungen, Datenquellen vgl. Tabelle A2 und A3.

\subsection{Zusammenfassung}

Der Effekt der Teilnahme an Agrarumweltmaßnahmen und an der Ausgleichszulage auf das betriebliche Flächenwachstum wurde mit Hilfe verschiedener ökonometrischer Panelmodelle (gepooltes OLS-Modell, FE-Modelle, dynamische GMM-Modelle) untersucht. Das gepoolte OLS-Modell und das Fixed-Effekt-Modell dienen als Referenz, um das Intervall eines relativ unverzerrten GMM-Schätzers eingrenzen zu können. Die verschiedenen Modelle werden hinsichtlich ihrer Annahmen und Ergebnisse miteinander verglichen.

Die Überprüfung der Modellannahmen zeigte, dass je nach Modell unterschiedliche Annahmeverletzungen auftreten. Ein Vorteil von FE- und GMM-Modellen besteht in der Möglichkeit, den Einfluss unbeobachteter Faktoren auf das interessierende Ergebnis zu kontrollieren (selection on unobservables). Gepoolte OLS-Modelle erlauben dagegen nur die Kontrolle des Einflusses beobachteter Faktoren (selection on observables). Ein Nachteil des FE-Modells ist die Annahme strikter Exogenität. Diese Annahme kollidiert mit einer für die empirische Fragestellung wünschenswerten dynamischen Modellspezifikation. Des Weiteren besteht zwischen dem Flächenwachstum und möglichen betrieblichen Determinanten (z. B. Arbeits- und Kapitaleinsatz) ein simultan kausaler Zusammenhang. Die Annahme strikter Exogenität dürfte daher in mikroökonomischen Studien häufig nur schwer zu erfüllen sein. In GMM-Modellen wird diesem Problem durch die Verwendung interner Instrumente, in Form früherer Lags des endogenen Regressors, begegnet. 
Die empirischen Ergebnisse zeigen, dass Agrarumweltmaßnahmen einen signifikant positiven Effekt auf das betriebliche Flächenwachstum haben. Betriebe mit AUM-Teilnahme weisen im Durchschnitt und pro Jahr ein um 0,6 \% höheres Flächenwachstum auf als vergleichbare Betriebe ohne Programmteilnahme. Das höhere Flächenwachstum der Teilnehmerbetriebe ist auf die Flächenbindung der AUM-Zahlungen zurückzuführen: Durch die flächengebundenen AUM-Zahlungen verbessert sich die Entlohnung des eingesetzten Bodens. Die Nutzung von Boden wird, im Vergleich zu anderen Faktoren, günstiger. Unter dem Prinzip der Gewinnmaximierung besteht daher ein Anreiz, den Einsatz des Bodens zu erhöhen (seine Verfügbarkeit vorausgesetzt). Der Erhalt der Ausgleichzulage hat keinen signifikanten Effekt auf das betriebliche Flächenwachstum.

Im zweiten Teil dieses Kapitel wurde der Frage nachgegangen, ob der Effekt der AUMund AZ-Teilnahme auf das Flächenwachstum in Abhängigkeit von betrieblichen und regionalen Merkmalen variiert. Die Höhe der Programmzahlungen kann als Indikator des flächenmäßigen Teilnahmeumfangs interpretiert werden: Mit steigenden AUM-Zahlungen je Betrieb nimmt der Effekt auf das Flächenwachstum zu. Von diesem positiven Effekt profitieren insbesondere Acker- und Futterbaubetriebe, Betriebe von älteren Betriebsleitern und Betriebe mit einer hohen Teilnahmewahrscheinlichkeit. Der Effekt der AUM-Teilnahme auf das betriebliche Flächenwachstum variiert in Abhängigkeit von der Betriebsform, der Betriebsgröße und vom Bundesland unsystematisch. Der insgesamt geringe Einfluss der Ausgleichszulage auf das betriebliche Flächenwachstum bestätigt sich auch in der Analyse heterogener Programmeffekte. 


\section{Diskussion und Ausblick}

Im Kapitel 10 werden die empirischen Ergebnisse zusammenfassend dargestellt (Kapitel 10.1) und die Vor- und Nachteile der verwendeten Methoden diskutiert (Kapitel 10.2). Hieraus ergeben sich Ansatzpunkte zur Verbesserung (agrar-)politischer Wirkungsanalysen (Kapitel 10.3).

\subsection{Empirische Ergebnisse}

In der Arbeit wurden die Effekte der Teilnahme an Agrarumweltmaßnahmen (AUM) und an der Ausgleichszulage (AZ) auf den betrieblichen Faktoreneinsatz (Boden, Arbeit, Kapital, Vieh, Pflanzenschutz- und Düngemittel), die Produktion (Umsatz) und den außerbetrieblichen Arbeitseinsatz des Betriebsleiterpaares im Zeitraum von 2000 bis 2005 untersucht.

Die empirische Analyse basiert auf einem Datensatz mit etwa 23.000 buchführenden Betrieben aus Deutschland. Die Stichprobe umfasst damit etwa $5 \%$ aller landwirtschaftlichen Betriebe in Deutschland. Große Haupterwerbsbetriebe aus den Alten Bundesländern sind in den Daten überrepräsentiert. Die Ergebnisse und Schlussfolgerungen gelten daher nur für die Betriebe der verwendeten Stichprobe. Aussagen zum Einfluss von Agrarumweltmaßnahmen und der Ausgleichszulage in Nebenerwerbsbetrieben, in ostdeutschen Betrieben und in kleinen Betrieben (unter 20 ha LF) sind auf Basis dieser Analyse nicht möglich.

Für die empirische Analyse wurden ein semi-parametrischer Matching-Ansatz (Propensity Score Matching) und verschiedene Panelregressionsmodelle verwendet. In der Matching-Analyse werden Paare aus vergleichbaren Teilnehmern und Nichtteilnehmern ${ }^{68}$ (= Kontrollbetriebe) gebildet und hinsichtlich ihrer Änderung des Faktoreinsatzes im Zeitraum von 2000 bis 2005 miteinander verglichen. Vergleichbare Betriebe wurden auf Basis eines aggregierten Ähnlichkeitsmaßes (dem Propensity Score) bestimmt. In der Panelanalyse wird der Einfluss der AUM- und AZ-Teilnahme auf das interessierende Ergebnis, unter Kontrolle „sonstiger“ Einflussfaktoren, modelliert. Es wurden mehrere Panelmodelle (statische, dynamische) mit variierenden Annahmen spezifiziert und hinsichtlich ihrer Annahmen und Ergebnisse miteinander verglichen. In der MatchingAnalyse wird der Einfluss der Programmteilnahme auf alle genannten Ergebnisvariablen

68 AUM-Teilnehmer haben im Referenzjahr (2000) nicht und von 2001 bis 2005 kontinuierlich an AUM teilgenommen (d. h. einen positiven Betrag Agrarumweltzahlungen erhalten). AZ-Teilnehmer haben von 2000 bis 2005 kontinuierlich eine Ausgleichszulage erhalten. Kontrollbetriebe haben in keinem Jahr zwischen 2000 und 2005 Agrarumweltzahlungen bzw. eine Ausgleichszulage erhalten. 
untersucht, während in der Panelanalyse nur der Effekt der Programmteilnahme auf das betriebliche Flächenwachstum analysiert wird.

Tabelle 10.1 stellt den mittleren Effekt der Teilnahme an Agrarumweltmaßnahmen und an der Ausgleichszulage, bezogen auf alle untersuchten Betriebe, dar. Die Teilnahme an Agrarumweltmaßnahmen hat einen signifikant positiven Einfluss auf das betriebliche Flächenwachstum $(L F)$. Die jährliche Wachstumsrate der landwirtschaftlich genutzten Fläche ist in Teilnehmerbetrieben um 1,0\% (Matching-Analyse) bzw. 0,8\% (Panelregression) höher als in den Kontrollbetrieben (= vergleichbare Betriebe ohne Teilnahme). Der Einfluss der Ausgleichszulage auf das betriebliche Flächenwachstum ist mit 0,4 \% bzw. 0,2 \% deutlich geringer und nur für den gesamten Untersuchungszeitraum betrachtet $(2000$ bis 2005) signifikant. Die Ergebnisse der Matching-Analyse und der Panelregression sind, bezogen auf das betriebliche Flächenwachstum, nahezu identisch, obwohl methodisch bedingt keine identischen Stichproben verwendet wurden. Dies untermauert die Plausibilität der Ergebnisse und die Eignung beider Methoden zur Wirkungsanalyse agrarpolitischer Programme.

Der positive Effekt von Agrarumweltmaßnahmen auf das betriebliche Flächenwachstum in Deutschland ist bereits durch OSTERBURG und STRATMANN (2002) belegt und deckt sich allgemein mit der Beobachtung, dass ge- und entkoppelte Direktzahlungen einen positiven Effekt auf die bewirtschaftete Fläche haben (vgl. Literaturüberblick in Tabelle 4.1). Da die Höhe der Programmzahlung primär an den Faktor Boden gebunden ist, stellt die Ausweitung der förderfähigen Flächen eine Strategie der Gewinnmaximierung dar - die Betriebe wachsen über die Fläche statt über die Nutzungsintensität. Diese These wird insbesondere für Agrarumweltmaßnahmen untermauert, da sich die Faktorintensität (Rinderbesatzdichte, Ausgaben Dünger je ha, AK Betrieb je 100 ha) und damit auch die Produktivität (Umsatz je ha) infolge der AUM-Teilnahme signifikant verringert.

Der positive Effekt von Agrarumweltmaßnahmen auf das betriebliche Flächenwachstum in Deutschland ist bereits durch OSTERBURG und STRATMANN (2002) belegt und deckt sich allgemein mit der Beobachtung, dass ge- und entkoppelte Direktzahlungen einen positiven Effekt auf die bewirtschaftete Fläche haben (vgl. Literaturüberblick in Tabelle 4.1). Da die Höhe der Programmzahlung primär an den Faktor Boden gebunden ist, stellt die Ausweitung der förderfähigen Flächen eine Strategie der Gewinnmaximierung dar - die Betriebe wachsen über die Fläche statt über die Nutzungsintensität. Diese These wird insbesondere für Agrarumweltmaßnahmen untermauert, da sich die Faktorintensität (Rinderbesatzdichte, Ausgaben Dünger je ha, AK Betrieb je 100 ha) und damit auch die Produktivität (Umsatz je ha) infolge der AUM-Teilnahme signifikant verringert. 
Tabelle 10.1: Differenz der mittleren jährlichen Ergebnisänderung (2000 bis 2005, in Prozent) zwischen Betrieben mit und ohne AUM- bzw. AZ-Teilnahme

\begin{tabular}{|c|c|c|c|c|c|}
\hline \multirow[t]{2}{*}{ Ergebnisvariable } & \multirow[t]{2}{*}{ Einheit } & \multicolumn{2}{|c|}{ Agrarumweltmaßnahmen } & \multicolumn{2}{|c|}{ Ausgleichszulage } \\
\hline & & $\begin{array}{l}\text { Matching } \\
\text { Differenz p }\end{array}$ & $\begin{array}{l}\text { Panelregression } \\
\text { Differenz p }\end{array}$ & $\begin{array}{c}\text { Matching } \\
\text { Differenz p }\end{array}$ & $\begin{array}{c}\text { Panelregression } \\
\text { Differenz p }\end{array}$ \\
\hline $\ln L F$ & $\Delta \%$ & $1,0 * * *$ & $0,8 * * *$ & $0,4 * *$ & 0,2 \\
\hline ln Grünland & $\Delta \%$ & $2,3 * * *$ & n.u. & $2,1 * * *$ & n.u. \\
\hline In Anteil Pachtland & $\Delta \%$ & $1,2 *$ & n.u. & $1,1 *$ & n.u. \\
\hline $\ln$ AK Betrieb & $\Delta \%$ & 0,1 & n.u. & 0,1 & n.u. \\
\hline ln AK Betrieb (je 100 ha) & $\Delta \%$ & $-0,9 * * *$ & n.u. & $-0,3$ & n.u. \\
\hline $\ln$ Außerbetriebliche $A K$ & $\Delta \%$ & $-0,3$ & n.u. & $-1,3$ & n.u. \\
\hline In Kapital (je ha) & $\Delta \%$ & $-0,5$ & n.u. & $-0,7 * *$ & n.u. \\
\hline ln Rinderbesatz & $\Delta \%$ & $-0,8$ & n.u. & 0,8 & n.u. \\
\hline In Rinderbesatzdichte & $\Delta \%$ & $-1,3 * * *$ & n.u. & $-1,3 * * *$ & n.u. \\
\hline ln Ausgaben PSM (je ha) & $\Delta \%$ & 0,9 & n.u. & 0,2 & n.u. \\
\hline ln Ausgaben Dünger (je ha) & $\Delta \%$ & $-1,3 * *$ & n.u. & $-0,4$ & n.u. \\
\hline $\ln$ Umsatz & $\Delta \%$ & 0,4 & n.u. & 0,4 & n.u. \\
\hline ln Umsatz (je ha) & $\Delta \%$ & $-0,7 * *$ & n.u. & $-0,1$ & n.u. \\
\hline
\end{tabular}

1. n. u. = nicht untersucht.

2. Signifikanzniveau: $* * * \mathrm{p}<0,01, * * \mathrm{p}<0,05, * \mathrm{p}<0,1$.

3. In der Matching-Analyse wurde die Ergebnisänderung für den Zeitraum 2000 bis 2005 geschätzt. In Tabelle 10.1 ist die auf ein Jahr normalisierte Änderungsrate des Ergebnisses dargestellt. Das Signifikanzniveau (p) bezieht sich auf den untersuchten Zeitraum 2000 bis 2005.

4. Das Ergebnis der Panelregression entspricht dem Ergebnis des Modells GMM-OR.

Quelle: Zusammenstellung auf Basis der Tabellen 8.3 und 9.2.

Der deutlich geringere Effekt der Ausgleichszulage auf das Flächenwachstum ist ebenfalls plausibel zu begründen: Zum einen sind die mittleren Prämienhöhen der Ausgleichszulage in etwa halb so groß wie die von Agrarumweltmaßnahmen. Dementsprechend ist der ökonomische Anreiz zur Änderung des Bodeneinsatzes geringer. Zum anderen ist die durch die Ausgleichszulage potenziell förderfähige Fläche durch die Gebietskulisse (benachteiligte Gebiete) festgelegt. Neu gepachtete Flächen sind nur förderfähig, soweit diese innerhalb der Gebietskulisse liegen. Im Gegensatz hierzu kann ein AUM-Teilnehmer auch für neu gepachtete Flächen AUM-Zahlungen erhalten.

Der signifikant positive Effekt der AUM-Teilnahme auf das betriebliche Flächenwachstum kann als ein Indiz für den komparativen Vorteil von Teilnehmerbetrieben auf dem Bodenmarkt gewertet werden. Diese Hypothese wird auch durch Aussagen landwirtschaftlicher Berater gestützt, die die gestiegene Flächennachfrage seitens der AUM-Teilnehmer als ein Hemmnis der betrieblichen Entwicklung für nicht teilnehmende Betriebe ansehen (REITER et al., 2003a). 
In der Matching-Analyse wurde der Effekt der AUM- und AZ-Teilnahme auf den Einsatz weiterer betrieblicher Faktoren (Arbeit, Kapital, Vieh, Dünge- und Pflanzenschutzmittel) auf den Umsatz sowie auf die außerbetriebliche Erwerbsbeteiligung des Betriebsleiterpaares untersucht. Die wesentlichen Ergebnisse sind:

- Infolge des überproportionalen Flächenwachstums verringert sich die Kapitalintensität (Kapital je ha) in AZ-Betrieben um jährlich 0,7\%. Ein vergleichbarer Effekt ist für die Teilnehmer an Agrarumweltmaßnahmen nicht zu beobachten. Ursachen hierfür könnten sein, dass AZ-Teilnehmer über weniger Kapital für betriebliche Investitionen verfügen oder Investitionen - stärker als dies ohnehin der Fall ist zunehmend im außerlandwirtschaftlichen Bereich tätigen.

- Der Arbeitseinsatz je Betrieb (AK Betrieb) sowie der außerbetriebliche Arbeitseinsatz des Betriebsleiterpaares (Außerbetriebliche $A K$ ) werden durch die AUM- bzw. AZTeilnahme nicht signifikant beeinflusst. Bezogen auf den Arbeitseinsatz sind weder ein Substitutions- noch ein Einkommenseffekt zu beobachten. Dies deckt sich mit Ergebnissen von GoodwiN et al. (2007) und AHEARN et al. (2005), die für das Conservation Reserve Program ebenfalls keinen Effekt auf die außerbetriebliche Erwerbsbeteiligung feststellen konnten.

- Betriebe mit Ausgleichszulage verringern den betrieblichen Kapitaleinsatz im Vergleich zur Kontrollgruppe um jährlich 0,7 \%. Ursachen hierfür könnten sein, dass AZTeilnehmer über weniger Kapital für betriebliche Investitionen verfügen oder Investitionen - stärker als dies ohnehin der Fall ist - zunehmend im außerlandwirtschaftlichen Bereich tätigen.

- Der Effekt beider Programme (AUM, AZ) auf den betrieblichen Umsatz ist nicht signifikant von Null verschieden. Im Durchschnitt aller Betriebe ist ein produktionsfördernder Effekt der analysierten Programme statistisch nicht belegbar. Im Fall von Agrarumweltmaßnahmen wird der Umsatzrückgang infolge verringerter Produktivität (Umsatz je ha) durch das betriebliche Flächenwachstum kompensiert. Das Flächenwachstum infolge der Ausgleichszulage ist vermutlich zu gering, um - bei konstanter Produktivität - eine Zunahme der Produktion bewirken zu können.

- Beide Maßnahmen (AUM, AZ) haben einen signifikant positiven Effekt auf die betriebliche Grünlandentwicklung: AUM- und AZ-Teilnehmer wachsen zu etwa gleichen Teilen über Grünland- und Ackerflächen, während Kontrollbetriebe fast ausschließlich über Ackerflächen wachsen. Der Effekt der Programmteilnahme bezogen auf das betriebliche Grünland beträgt für Agrarumweltmaßnahmen $+2,3 \%$ und die Ausgleichszulage $+2,1 \%$ pro Jahr. Der Grünlandrückgang in den Kontrollbetrieben legt nahe, dass AUM- und AZ-Teilnehmer Grünlandflächen von nicht teilnehmenden Betrieben übernehmen. Ob dieses Grünland in der Situation ohne AUM und AZ teilweise umgebrochen worden wäre, ist auf Basis dieser betriebsbezogenen Analyse nicht zu klären. Der Beitrag von Agrarumweltmaßnahmen und der Ausgleichszulage zum Grünlanderhalt kann nur in einer flächenbezogenen Analyse untersucht werden. 
- Die Teilnahme an AUM und AZ führt zu einer signifikanten Verringerung des Rinderbestandes je Hauptfutterfläche (Rinderbestandsdichte) (-1,3\% pro Jahr im Vergleich zur Kontrollgruppe). Die Reduzierung der Rinderbestandsdichte ist vorrangig auf die Ausweitung der LF zurückzuführen, da der Rinderbestand je Betrieb durch die Programmteilnahme nicht beeinflusst wird.

- Die AUM-Teilnahme ist mit einer signifikanten Reduzierung der Düngemittelausgaben je Hektar LF (Ausgaben Dünger je ha) verbunden. Zwischen 2000 und 2005 reduzierten sich die Düngemittelausgaben in Teilnehmerbetrieben im Vergleich zu Kontrollbetrieben um 6,4\%. ${ }^{69}$ Unter der Annahme, dass ausschließlich Stickstoffdünger ${ }^{70}$ verwendet wird, entspricht dies einer Reduzierung von etwa $9 \mathrm{~kg}$ Stickstoff je Hektar LF und Jahr. Für die AZ ist kein vergleichbarer Effekt zu beobachten. Beide untersuchten Programme haben keinen signifikanten Einfluss auf Ausgaben für Pflanzenschutzmittel je Hektar (Ausgaben PSM je ha).

Bisher wurden die durchschnittlichen Effekte der AUM- und AZ-Teilnahme betrachtet. Inwieweit die Effekte der Programmteilnahme in Abhängigkeit von betrieblichen und regionalen Merkmale variieren, wurde in der Analyse heterogener Programmeffekte untersucht.

\section{Heterogene Effekte von Agrarumweltmaßnahmen und der Ausgleichszulage}

Aufgrund der unterschiedlichen Umsetzung der Programme in den deutschen Bundesländern, der variierenden Inanspruchnahme seitens der Betriebe und der betriebsindividuellen Anpassungsstrategien an die Bedingungen der Programmteilnahme ist $\mathrm{zu}$ erwarten, dass die Effekte von Agrarumweltmaßnahmen und der Ausgleichszulage nicht für alle Teilnehmer gleich sind. Die Effekte der AUM- und AZ-Teilnahme wurden in Abhängigkeit von der Höhe der Programmzahlungen (je Betrieb bzw. je Hektar LF), der Betriebsgröße (LF), der Betriebsform, der Wahrscheinlichkeit der Programmteilnahme, dem Alter des Betriebsleiters und dem Bundesland untersucht.

Die vermutlich größten Unterschiede hinsichtlich der Programmeffekte sind in Abhängigkeit von der Art der Agrarumweltmaßnahme (Grünlandextensivierung etc.), der geförderten Fläche je Betrieb und der Prämienhöhe je Hektar zu erwarten. Diese Aspekte konnten in der Analyse heterogener Programmeffekte nicht berücksichtigt werden, da die verwendeten Buchführungsdaten hierzu keine Informationen enthalten.

69 Der in Tabelle 10.1 ausgewiesene Effekt in Höhe von - 1,3 \% (Änderungsrate pro Jahr) ist in Bezug auf Düngemittelausgaben missverständlich, da es sich um jährlich wiederkehrende Ausgaben handelt.

70 Kalkammonsalpeter (26\% Stickstoff, Schüttgut), Preis je 100 kg Nährstoff: 49,00 Euro (2000) bzw. 62,01 Euro (2005) (EUROSTAT, 2006). 
Die empirischen Ergebnisse bestätigen die These heterogener Programmeffekte und sind zusammenfassend in Tabelle 10.2 dargestellt. Statistisch signifikante Programmeffekte in Abhängigkeit betrieblicher und regionaler Merkmale sind vor allem für jene Ergebnisvariablen zu beobachten, für die bereits in Tabelle 10.1 signifikante Programmeffekte festzustellen waren (LF, Grünland, Rinderbesatzdichte, Ausgaben Dünger je ha).

Tabelle 10.2: Heterogenität der Effekte der AUM- und AZ-Teilnahme in Abhängigkeit von betrieblichen und regionalen Merkmalen

\begin{tabular}{|c|c|c|c|c|}
\hline \multirow{2}{*}{$\begin{array}{l}\text { Effekt der Programmteile ist abhängig } \\
\text { von der/dem * }\end{array}$} & \multicolumn{2}{|c|}{ Agrarumweltmaßnahmen } & \multicolumn{2}{|c|}{ Ausgleichszulage } \\
\hline & Matching & Panelregression & Matching & Panelregression \\
\hline Höhe der Programmzahlungen & ja & ja & ja & ja \\
\hline Bundesland, Region & ja & ja & ja & ja \\
\hline Betriebsform & ja & ja & ja & nein \\
\hline Betriebsgröße & ja & ja & ja & ja \\
\hline Wahrscheinlichkeit der Programmteilnahme & ja & ja & ja & ja \\
\hline Alter des Betriebsleiters & n.u. & ja & n.u. & nein \\
\hline Anteil LF im benachteiligten Gebiet & n.u. & ja & n.u. & ja \\
\hline
\end{tabular}

* bezogen auf mindestens eine Ergebnisvariable, n. u. = nicht untersucht.

Quelle: Zusammenstellung auf Basis der Ergebnisse der Kapitel 8.4 und 9.3.

Die Höhe der AUM- und AZ-Zahlungen (= Programmzahlungen) je Betrieb wird als Proxy für den Umfang der Programmteilnahme verwendet. Betriebe mit hohen Programmzahlungen bewirtschaften einen hohen Anteil ihrer betrieblichen LF im Rahmen von Agrarumweltmaßnahmen bzw. der Ausgleichszulage. Die Ergebnisse der MatchingAnalyse und der Panelanalyse zeigen, dass die Effekte der AUM-Teilnahme mit steigenden Programmzahlungen zunehmen ( $L F$, Grünland, Kapiteleinsatz, Rinderbesatzdichte). Die Effekte der AZ-Teilnahme variieren ebenfalls in Abhängigkeit von den Programmzahlungen. Ein linearer Zusammenhang zwischen dem Niveau des Effektes und der Höhe der Programmzahlungen ist, mit einer Ausnahme (Rinderbestand), jedoch nicht erkennbar.

Der Einfluss der AUM- und AZ-Teilnahme auf das betriebliche Flächenwachstum und den Einsatz anderer betrieblicher Faktoren ist nicht in allen Bundesländern bzw. Regionen (Nord, West, Süd, Ost) gleich. Die Effekte der Ausgleichszulage variieren zwischen den Bundesländern weniger stark als die Effekte von Agrarumweltmaßnahmen. Dies dürfte auf die vergleichsweise einheitliche Umsetzung der Ausgleichszulage in den Bundesländern zurückzuführen sein. Die Ausgleichszulage wird in Deutschland ausschließlich im Rahmen der GAK umgesetzt, während die Agrarumweltprogramme der Länder sowohl GAKals auch Nicht-GAK-Maßnahmen umfassen. Ein typisches Muster der Programmeffekte je Bundesland ist nicht zu beobachten. Die Vermutung, dass AUM-Teilnehmer in Bundes- 
ländern mit einer relativ hohen Agrarumweltförderung je Hektar (z. B. BadenWürttemberg, Sachsen) vergleichsweise hohe Programmeffekte aufweisen, wird durch die empirischen Ergebnisse nicht gestützt.

An Agrarumweltmaßnahmen teilnehmende Acker- und Futterbaubetriebe weisen im Zeitraum 2000 bis 2005 ein höheres Flächenwachstum auf als teilnehmende Veredlungs- und Gemischtbetriebe. Das höhere Flächenwachstum in Acker- und Futterbaubetrieben dürfte insbesondere auf die Einführung der Modulationsmaßnahmen (Mulch- und Direktsaatverfahren, Winterbegrünung, emissionsarme Gülleausbringung) im Jahr 2003 zurückzuführen sein. Vor diesem Zeitpunkt waren die Effekte der AUM-Teilnahme für alle Betriebsformen gleich. Nach 2003 ist vor allem für Futterbau eine signifikante Zunahme der LF aufgrund von Agrarumweltmaßnahmen zu beobachten (+ 1,6 \% pro Jahr). Die Beobachtung, dass Acker- und Futterbaubetriebe überproportional von der AUM-Teilnahme profitieren, könnte darauf zurückzuführen sein, dass der Nutzen von Agrarumweltmaßnahmen (z. B. in Form des Flächenwachstums) in spezialisierten Betrieben vergleichsweise hoch ist, da viele angebotene AUM entweder auf den Betriebszweig Ackerbau (z. B. Mulch- und Direktsaatverfahren, Winterbegrünung) oder auf den Betriebszweig Grünlandwirtschaft (z. B. Grünlandextensivierung, Weidehaltung) ausgerichtet sind.

Die empirischen Ergebnisse zum Einfluss der Betriebsgröße auf die Höhe der Programmeffekte ergeben ein widersprüchliches Bild. Zwar variieren die Programmeffekte zwischen Teilnehmern unterschiedlicher Betriebsgröße. Ein systematischer (z. B. linearer) Zusammenhang zwischen der Betriebsgröße und dem Programmeffekt ist jedoch nicht festzustellen. Je nach Untersuchungsmethode ist z. B. das Flächenwachstum von AUM-Teilnehmern unabhängig von der Betriebsgröße (Matching-Analyse), positiv korreliert mit der Betriebsgröße (Panelanalyse, POLS II, FE II-Modelle) oder negativ korreliert mit der Betriebsgröße (Panelanalyse, GMM-Modelle). Ähnlich variierende Ergebnisse sind auch für die Ausgleichszulage zu beobachten. Aufgrund der Widersprüchlichkeit der Ergebnisse ist vorerst von keinem gesicherten Zusammenhang zwischen der Betriebsgröße und dem Effekt von AUM auf das Flächenwachstum auszugehen. Dies deckt sich mit den Aussagen des Gesetzes von Gibrat, das die Unabhängigkeit zwischen der Betriebsgröße und dem betrieblichen Wachstum postuliert.

Ein positiver Zusammenhang besteht zwischen dem Effekt der Programmteilnahme und der Wahrscheinlichkeit der Teilnahme an Agrarumweltmaßnahmen bzw. an der Ausgleichszulage. Die Wahrscheinlichkeit der Programmteilnahme wird auf Basis beobachteter, betrieblicher Merkmale (z. B. Bodennutzung, Nutzungsintensität, Standortqualität) geschätzt. Teilnehmerbetriebe mit einer hohen Teilnahmewahrscheinlichkeit können einen vergleichsweise großen Nutzen aus der Programmteilnahme, z. B. bezogen auf das Flächenwachstum, ziehen. Dieses Ergebnis untermauert, dass die freiwillige Entscheidung zur Programmteilnahme (insbesondere an AUM) eine ökonomisch rationale Entscheidung 
der Betriebsleiter ist. Betriebe, die aufgrund ihrer betrieblichen Situation einen positiven Nutzen aus der AUM-Teilnahme ziehen können, werden sich für die Programmteilnahme entscheiden. Da ein hoher Umweltnutzen von AUM nicht notwendiger Weise mit einem hohen betrieblichen Nutzen einhergeht, ist das Prinzip „Selbstselektion zur AUMTeilnahme“" kein effektives Steuerungsinstrument für Agrarumweltmaßnahmen.

Ältere Betriebsleiter nehmen besonders häufig an Agrarumweltmaßnahmen teil. Gründe hierfür können die Reduzierung der Arbeitsbelastung oder der Verzicht auf betriebliche Investitionen mit Blick auf das nahe Rentenalter sein. Auch ohne Agrarumweltmaßnahmen hätten ältere Betriebsleiter eventuell betriebliche Anpassungen vorgenommen. Die Entscheidungen zur AUM-Teilnahme und zur Reduzierung der Arbeitsbelastung sind daher vermutlich nicht unabhängig (vgl. CHANG und BOISVERT, 2005). Die Opportunitätskosten der Reduzierung der Arbeitsbelastung bzw. des Verzichtes auf Investitionen werden teilweise durch AUM-Zahlungen kompensiert. Der Nutzen der AUM-Teilnahme, z. B. in Form des betrieblichen Flächenwachstums, ist daher in Betrieben mit älteren Betriebsleitern deutlich höher als in Betrieben mit jüngeren Betriebsleitern. Das Ergebnis legt nahe, dass ältere Betriebsleiter Agrarumweltmaßnahmen teilweise zum ,ausgleiten“ aus der landwirtschaftlichen Erwerbstätigkeit nutzen.

Abweichend von den in Tabelle 10.1 dargestellten mittleren Programmeffekten, zeigt die Analyse heterogener Effekte einen signifikant positiven Einfluss von Agrarumweltmaßnahmen auf den betrieblichen Arbeitseinsatz in Veredlungsbetrieben und auf die Produktion in Futterbaubetrieben, mittleren (20 bis 50 ha LF) und großen Betrieben (über 100 ha LF) sowie in Betrieben mit geringen AUM-Zahlungen (unter 25 Euro je ha LF). Die Effekte der Ausgleichszulage auf den betrieblichen (außerbetrieblichen) Arbeitseinsatz sind in Ackerbaubetrieben signifikant positiv (negativ), so dass hier von einem Substitutionseffekt zwischen außerbetrieblicher und betrieblicher Arbeit auszugehen ist. Die Ausgleichszulage hat ferner einen produktionsfördernden Effekt in süddeutschen Betrieben und Betrieben mit geringen AZ-Zahlungen (unter 25 Euro je ha LF).

\subsection{Vor- und Nachteile der verwendeten Methoden}

Für die Quantifizierung des kausalen Effektes der Programmteilnahme wurden in dieser Arbeit ein Matching-Ansatz und verschiedene Panelregressionsmodelle verwendet. Die Vor- und Nachteile von Matching-Verfahren und parametrischen (ökonometrischen) Modellen in der Politikevaluation wurden z. B. von HECKMAN und NAVARRO-LOZANo (2004) und HeCKMAN und VytLACIL (2007a) diskutiert. Auch in der deutschsprachigen agrarökonomischen Literatur ist ein Austausch über das Für und Wider beider methodischer Ansätze entstanden (HeNning und MichaleK, 2008a, 2008b, MARGarian, 2008). 
Um die sehr facettenreiche Diskussion zu strukturieren und auf die wesentlichen Aspekte zu beschränken, werden beide methodischen Ansätze (Matching-Verfahren, ökonometrische Modelle) hinsichtlich ihrer Annahmen, der Kontrolle unbeobachteter Effekte, der Berücksichtigung dynamischer Aspekte, des Datenbedarfs, der Praktikabilität und Flexibilität diskutiert.

\section{Annahmen}

Ausgangspunkt der Kritik an parametrischen Evaluationsmethoden sind deren restriktive Annahmen hinsichtlich der korrekten Spezifikation des empirischen Modells. Diese Annahmen betreffen v. a. die funktionale Form des Modells, die Verteilung und Exogenität der Regressoren, die Verfügbarkeit valider Instrumente sowie die Annahme additiv separabler und homogener Programmeffekte (WoOLDRIDGE, 2002 HECKMAN und VYTLACIL, 2007a, HENNING und MichALEK, 2008a). Sind diese Annahmen nicht erfüllt ${ }^{71}$, so können die geschätzten Ergebnisse verzerrt sein, was wiederum zu falschen Schlussfolgerungen hinsichtlich der Politikwirkung führen kann.

Der Matching-Ansatz, als ein Beispiel für nicht- bzw. semi-parametrische Verfahren ${ }^{72}$, basiert auf weniger restriktiven Annahmen als parametrische Methoden. So bleibt z. B. der funktionale Zusammenhang zwischen der Politikvariablen und dem interessierenden Ergebnis unspezifiziert (HECKMAN und VyTLACIL, 2007a). ${ }^{73}$ Der Effekt der Programmteilnahme kann zwischen Teilnehmern mit unterschiedlichen Charakteristika variieren und muss nicht additiv separabel sein (HECKMAN und NAVARRo-LozAnO, 2004). Der Matching-Ansatz ist damit robuster gegenüber Aspekten, die bei parametrischen Verfahren zu Annahmeverletzungen führen. Die Kosten hierfür sind ein hoher Datenbedarf (siehe unten) und lediglich theoretisch überprüfbare Annahmen.

Die Stable Unit Treatment Value Assumption (SUTVA) schließt z. B. das Vorhandensein allgemeiner Gleichgewichtseffekte aus. Anpassungen auf Produkt- und Faktormärkten als Folge agrarpolitischer Maßnahmen beeinflussen jedoch häufig auch Betriebe, die nicht unmittelbar an dem betrachteten Programm teilnehmen. Die empirischen Ergebnisse dieser Arbeit zeigen, dass sich infolge von Agrarumweltmaßnahmen der Bodeneinsatz in den Teilnehmerbetrieben erhöht. Zumindest kleinräumig dürfte dies einen Effekt auf den Bo-

71

Die Annahmen können durch geeignete Modellspezifikation abgeschwächt werden. So kann z. B. die Annahme homogener Programmeffekte durch die Verwendung von Interaktionstermen weniger restriktiv gestaltet werden.

72 Alternative nicht- und semi-parametrische Verfahren, z. B. nicht-parametrische Regressionsschätzungen, wurden in dieser Arbeit nicht explizit behandelt. Einen Überblick über neuere Entwicklungen in diesem Bereich geben ICHIMURA und TODD (2007).

73 Im semi-parametrischen Propensity Score Matching wird von einem funktionalen Zusammenhang zwischen betrieblichen Merkmalen und der Entscheidung zur Programmteilnahme ausgegangen. 
denmarkt und auf benachbarte Betriebe haben. Eine Berücksichtigung dieser indirekten Effekte ist im Rahmen des Matching-Ansatzes nicht möglich. Diese Kritik gilt in gleicher Weise auch für parametrische Verfahren auf mikroökonomischer Ebene. Auch dort werden Rückkoppelungseffekte auf Güter- und Faktormärkte typischerweise ignoriert, es sei denn, die Analyse ist in ein umfangreiches empirisches Modell zur Abbildung des Gesamtsektors eingebunden.

Der Verzicht der Spezifikation eines formalen Modells setzt das Matching-Verfahren der Kritik der fehlenden theoretischen Fundierung aus. MARgarian (2008) sieht hierin die Gefahr, dass Matching-basierte Wirkungsanalysen - da methodisch möglich - ohne theoretische Fundierung durchgeführt werden. Die gewonnenen Ergebnisse würden einen $\mathrm{Zu}-$ fälligkeitscharakter erhalten, da deren Plausibilität nicht theoretisch überprüfbar ist. Im Kontext von Politikanalysen kann dies dazu führen, dass Argumente für den Erhalt einer Politik geliefert werden, obwohl diese Politik aufgrund einer fehlenden theoretischen Basis und dem Ausbleiben relevanter Wirkungen eigentlich keine Berechtigung hat. Die Kritik von HeCKMAN und VytLacil (2007a) an Matching-Ansätzen geht in die gleiche Richtung. Die Autoren bemängeln, dass in dem ursprünglichen Modell kausaler Effekte (RuBIN, 1974) weder die Auswahl der Ergebnisvariablen noch die Entscheidung zur Programmteilnahme vor dem Hintergrund der sozialen oder ökonomischen Theorie diskutiert werden.

Grundsätzlich ist diese Kritik richtig und berechtigt. Allerdings stellt sich auch hier die Frage nach der Kausalität. Ist die Ursache für nicht theoretisch fundierte MatchingAnalysen in der Methode selbst oder vielmehr in der praktischen Anwendung dieser Methode zu suchen? Die Anwendung des Matching-Verfahrens in dieser Arbeit zeigt, dass eine theoretische Diskussion der Bestimmungsgründe der AUM- und AZ-Teilnahme und deren Effekte möglich ist, wenn genügend Informationen über den institutionellen Rahmen und die inhaltliche Ausgestaltung der zu untersuchenden Programme vorliegen. Die theoretische Fundierung von Wirkungsanalysen ist somit keine Frage der verwendeten Methode, sondern ist von der jeweiligen Anwendung abhängig.

\section{Kontrolle unbeobachteter, individueller Effekte}

Das Handeln von Individuen, Haushalten und Betrieben wird durch eine Vielzahl von Faktoren beeinflusst, von denen die meisten Faktoren für den Wissenschaftler unbeobachtet (da nicht erhoben, nicht quantifizierbar und unbekannt) sind. Dies betrifft insbesondere individuelle Faktoren, wie Einstellungen, Motivation und Erfahrungen. Voraussetzungen für die Kontrolle unbeobachteter, individueller Effekte sind die Verfügbarkeit von Paneldaten und die Verwendung von Schätzern, die auf transformierten Daten (within-Schätzer, first differences etc.) beruhen (WOOLDRIDGE, 2002). 
Ein Nachteil des Matching-Ansatzes ist, dass die Auswahl von Teilnehmer- und Kontrollbetrieben ausschließlich auf Basis beobachteter Faktoren erfolgt (selection on observables). Wenn sich Teilnehmer- und Kontrollbetriebe bereits im Referenzjahr hinsichtlich ihrer unbeobachteten Merkmale unterscheiden und die unbeobachteten Merkmale in einem kausalen Zusammenhang mit dem interessierenden Ergebnis stehen, dann ist der geschätzte Effekt verzerrt. Im Differenz-in-Differenz Matching wird lediglich der Einfluss zeitkonstanter Faktoren auf das interessierende Ergebnis kontrolliert, nachdem die vergleichbaren Teilnehmer- und Kontrollbetriebe selektiert wurden. In Panelmodellen wird der Einfluss unbeobachteter, individueller Faktoren auf das Ergebnis unmittelbar durch die Schätzung auf Basis transformierter Daten kontrolliert (selection on unobservables) - allerdings nur, wenn der unbeobachtete Effekt zeitkonstant und additiv separabel ist (WoOLDRIDGE, 2002).

\section{Berücksichtigung dynamischer Aspekte}

Zur Untersuchung dynamischer Aspekte, wie z. B. der Veränderung des Faktoreinsatzes in landwirtschaftlichen Betrieben, ist eine angemessene Berücksichtung der zeitlichen Dimension notwendig. Die Verwendung von Methoden der Panelanalyse ist in dieser Hinsicht ein methodischer Fortschritt (gegenüber der Verwendung von Querschnittsdaten), berücksichtigt aber nicht automatisch die intertemporale Abhängigkeit von Entwicklungen.

Die Berücksichtung der Dynamik von (agrarstrukturellen) Entwicklungen ist durch die Verwendung dynamischer Panelmodelle möglich. Die in dieser Arbeit verwendeten GMM-Modelle sind insbesondere für die mikroökonomische Analyse gut geeignet, da diese - neben der dynamischen Modellierung - den Umgang mit endogenen Variablen und die Kontrolle unbeobachteter, individueller Effekte ermöglichen. Die entsprechenden Schätzer sind seit kurzem auch in der statistischen Standardsoftware (z. B. RoODMAN, 2006) verfügbar.

Die methodischen Entwicklungen im Bereich dynamischer Matching-Verfahren sind dagegen noch nicht so weit fortgeschritten. Zwar existieren bereits verschiedene Methoden und Anwendungsbeispiele um den Effekt mehrmaliger Programmteilnahmen und/oder der Teilnahmedauer zu berücksichtigen (vgl. LECHNER, 2004, ABBRING und V. D. BERG 2003). Die entsprechenden Methoden haben sich jedoch noch nicht etabliert und sind daher noch in keiner Standardsoftware verfügbar. 


\section{Datenbedarf}

Nicht- und semi-parametrische Verfahren wie das Matching nutzen die zur Verfügung stehenden Informationen weniger effizient als parametrische Verfahren und haben daher einen vergleichsweise hohen Datenbedarf.

Die Verfügbarkeit eines Datensatzes mit einer großen Anzahl an Beobachtungen und vielen Merkmalen ist eine Voraussetzung für die Anwendbarkeit des Matching. Das Verfahren wird daher primär für mikroökonomische Analysen (Betriebe, Personen, Haushalte) eingesetzt. Selbst auf der Basis eines vergleichsweise umfangreichen Datensatzes (23.120 Betriebe) hat sich in der vorliegenden Arbeit gezeigt, dass für viele Teilnehmer kein vergleichbarer Kontrollbetrieb gefunden werden konnte - einerseits, weil die meisten vergleichbaren Betriebe ebenfalls am Programm teilnehmen (Effekt der Selbstselektion), andererseits, weil mögliche Kontrollbetriebe in der Stichprobe nicht enthalten sind.

Der hierdurch bedingte Ausschluss von Teilnehmern ohne vergleichbaren „Zwilling“ aus der Analyse mag auf den ersten Blick eher als ein Nachteil des Matching erscheinen: je kleiner die Stichprobe, auf deren Basis die Ergebnisse gewonnen werden, desto weniger lassen sich die Ergebnisse verallgemeinern. Allerdings wird so sichergestellt, dass das hypothetische Ergebnis im Fall der Nichtteilnahme tatsächlich auf Basis vergleichbarer Betriebe ermittelt wird. Da in ökonometrischen Modellen in der Regel alle Beobachtungen verwendet werden, ist hier das Risiko von out of range predictions ungleich höher als im Matching.

\section{Praktikabilität der Methoden für die wissenschaftliche Politikbewertung}

Das Matching-Verfahren ist eine intuitiv sehr verständliche Methode (CAMERON und TRIVEDI, 2005: 872). Ergebnisse von Matching-Analysen sind sehr gut an ein nichtwissenschaftliches Publikum kommunizierbar, da Mittelwertvergleiche einfacher zu verstehen sind als durch parametrische Verfahren geschätzte Koeffizienten. Erfahrungen zeigen, dass kritische Evaluationsergebnisse insbesondere dann von politischen Entscheidungsträgern in Frage gestellt werden, wenn die Ergebnisse nicht nachvollziehbar sind. Um den Stellenwert der Politikevaluation zu erhöhen, sind Methoden, die theoretisch fundierte, valide und kommunizierbare Aussagen ermöglichen, notwendig.

\section{Flexibilität der Verfahren}

Flexibilität soll hier im Sinne der Anpassungsfähigkeit der Methoden an die zu untersuchende empirische Fragestellung verstanden sein.

Skalierung der Politikvariablen: Der Indikator der Programmteilnahme ist im Matching in der Regel binär skaliert, während parametrische Verfahren mehr Flexibilität bei der Skalierung der Politikvariablen (stetig, binär) erlauben. Die Verwendung stetiger oder 
ordinal skalierter Politikvariablen in der Matching-Analyse ist methodisch möglich (vgl. IMBENS, 2000; LECHNER, 2002), in der Regel aber aufwändig, da diese Methoden nicht in der Standardsoftware implementiert sind. Für die Evaluation von agrarpolitischen Instrumenten mit einer ausgeprägten Teilnehmer-/Nichtteilnehmerstruktur (Beratungsmaßnahmen, Agrarinvestitionsförderung, Agrarumweltmaßnahmen, Ausgleichszulage) ist daher der Matching-Ansatz gut geeignet. Für Wirkungsanalysen von Direktzahlungen, die nahezu jeder Betrieb erhält, sind eher parametrische Ansätze geeignet.

Hypothesentests: Im Matching wird in der Regel die Hypothese überprüft, ob das interessierende Ergebnis zwischen Individuen mit und ohne Programmteilnahme gleich ist. Ungleich aufwändiger ist im Matching der Test, ob die Effekte der Programmteilnahme zwischen verschiedenen Teilnehmergruppen variieren. ${ }^{74}$ Parametrische Verfahren bieten, aufgrund der verfügbaren Software, wesentlich flexiblere und zeitsparendere Möglichkeiten zur Überprüfung von Hypothesen.

Mittlere und marginale Effekte der Programmteilnahme: In der Politikevaluation ist zumeist der mittlere Effekt der Programmteilnahme von Interesse, der sowohl mit Hilfe von Matching- als auch mit parametrischen Verfahren geschätzt werden kann. Der marginale Effekt einer Politik lässt sich nur auf Basis parametrischer Verfahren ermitteln, da in der Matching-Analyse der mittlere und der marginale Effekte identisch sind (HECKMAN und VYTLACIL, 2007b).

Anzahl der zu untersuchenden Ergebnisvariablen: In einem ökonometrischen Modell wird der Einfluss verschiedener Faktoren auf ein interessierendes Ergebnis modelliert. Für jede zu untersuchende Ergebnisvariable muss ein Modell spezifiziert werden. In Verbindung mit der Überprüfung der Modellannahmen kann dies zeitaufwändig sein. Im Matching-Verfahren können dagegen mehrere Ergebnisvariablen gleichzeitig untersucht werden.

\section{Spezifische Eignung der Verfahren}

Neben den diskutierten Vor- und Nachteilen weisen Matching-Verfahren bzw. ökonometrische Modelle eine spezifische Eignung für die Wirkungsanalyse auf, die von der Art der verfügbaren Daten und der zu beantwortenden Fragestellung abhängt.

$74 \quad$ Der Effekt der Programmteilnahme wird für jede Teilnehmergruppe in einem separaten MatchingVerfahren geschätzt. Die Programmeffekte der einzelnen Teilnehmergruppen werden in einer separaten Datei zusammengefasst und paarweise verglichen (vgl. PUFAHL und WEISS, 2009). 
Verhältnis der Anzahl von Teilnehmer- und Kontrollbetrieben: Das MatchingVerfahren ist insbesondere dann geeignet, wenn die Gruppe der Teilnehmer im Vergleich zur Kontrollgruppe sehr klein ist. Nur in diesem Fall steht ein großer Pool mit genügend potenziellen Kontrollbetrieben zur Verfügung. ${ }^{75}$ Parametrische Verfahren sind vorteilhafter, wenn die Anzahl der Teilnehmer- und Kontrollbetriebe zahlenmäßig in etwa gleich groß ist.

Zeitliche Varianz der Politik: Matching-Verfahren im Zusammenhang mit Differenz-inDifferenz Schätzern sind nur sinnvoll anzuwenden, wenn im Untersuchungsraum genügend permanente (Nicht-) Teilnehmer vorhanden sind. Eine gewisse Konstanz des (Nicht-) Teilnahmeverhaltes ist für die Analyse also notwendig. Im Gegensatz dazu ist die zeitliche Varianz des Teilnahmeverhaltens eine zentrale Voraussetzung, um den Effekt der Programmteilnahme in Panelmodellen (mit transformierten Daten) zu ermitteln.

Beobachtbarkeit von Ergebnissen für Nichtteilnehmer: Der Matching-Ansatz kann nicht verwendet werden, wenn das interessierende Ergebnis nur für die Teilnehmer einer Politikmaßnahme zu beobachten ist. Soll z. B. der Nutzen einer neuen Technologie als Ergebnis einer Beratungsmaßnahme gemessen werden, so kann das potenzielle Ergebnis im Fall der Nichtteilnahme nicht geschätzt werden, wenn Betriebe ohne Beratung diese Technologie nicht einführen. In diesem Fall muss auf parametrische Selektionsmodelle in der Tradition von HECKMAN (1976) zurückgegriffen werden.

Intra- und interbetriebliche Varianz: Matching-Verfahren und Panelanalysen „schärfen" den Blick für unterschiedliche Varianzen in der Stichprobe. In Panelverfahren wird das interessierende Ergebnis als Mittelwert über alle Betriebe und alle Untersuchungszeitpunkte geschätzt. Graphische Ergebnisdarstellungen erfolgen daher meist zeitabhängig. Die zeitliche Entwicklung des Ergebnisses kann gut nachvollzogen werden, was z. B. zur Aufdeckung von Strukturbrüchen hilfreich sein kann. Im Matching wird der Blick eher für die Unterschiede zwischen Teilnehmern und Nichtteilnehmern geschärft, während der Zeitraum zwischen zwei Untersuchungszeitpunkten als Black Box behandelt wird.

Fragestellung der Evaluation: Wirkungsanalysen dienen in erster Linie der Quantifizierung von Programmeffekten und können mit Hilfe von Matching- und parametrischen Verfahren durchgeführt werden. Weitergehende Fragenstellungen, z. B. nach den Gründen der Programmteilnahme, hinsichtlich der Bedingungen, unter denen das Programm die erhofften Wirkungen erzielt oder Prognosen zu zukünftigen Wirkungen einer Politik lassen sich

75 Die Anzahl potenzieller Kontrollbetriebe (Nichtteilnehmer) sollte die der Teilnehmerbetriebe deutlich übersteigen. In dieser Arbeit wäre ein Verhältnis von Teilnehmern zu Nichtteilnehmern von $1: 4$ bis $1: 10$ wünschenswert gewesen. 
vor allem in Verbindung mit ökonometrischen Modellen beantworten (HECKMAN und VYTLACIL, 2007a).

Insbesondere die beiden letzten Punkte zeigen, dass die Wahl der Methoden in starkem Maß von der zu untersuchenden Fragenstellung und der Art der verfügbaren Daten abhängt. Im Wissen um die Stärken und Schwächen von Matching-Verfahren und parametrischen Analysemethoden eröffnet sich dem Wissenschaftler damit die Möglichkeit, die für die jeweilige Fragestellung geeignete Methode auszuwählen.

\subsection{Ausblick und Forschungsbedarf}

Aufgabe der wissenschaftlichen Politikanalyse ist es, die Kosten und den Nutzen (die Wirkungen) einer Politik in Hinblick auf deren gesellschaftlich relevante Ziele zu bestimmen und so Entscheidungsgrundlagen für eine rationale Politikgestaltung bereitzustellen. Die vorliegende Arbeit leistet einen methodischen Beitrag zur Wirkungsanalyse direkter Transferzahlungen allgemein und einen empirischen Beitrag hinsichtlich der Wirkungen von Agrarumweltmaßnahmen und der Ausgleichszulage auf den betrieblichen Faktoreinsatz und die Produktion. Der Ausblick fokussiert daher auf die methodischen und empirischen Aspekte der Wirkungsmessung direkter Transferzahlungen und lässt die ebenso wichtige Frage nach der Erhebung bzw. der Höhe der Kosten der Politik außer Acht.

\section{Methodik}

Der Vergleich der in der Literatur diskutierten und in der Agrarökonomie verwendeten Evaluationsmethoden zeigt die bestehenden Potenziale zur Verbesserung agrarökonomischer Wirkungsanalysen. In der praktischen Evaluation von Politikmaßnahmen geht es dabei weniger um die Anwendung der „neuesten Methoden“ als vielmehr um die Anwendung von Methoden, die allgemein als State of the Art gelten. Hierzu zählt z. B. die Analyse von Paneldaten mit etablierten Panelmethoden (Fixed-Effekt-Modelle, andere Differenz-in-Differenz Schätzer) und die statistische Absicherung von Mittelwertvergleichen.

Ein zweiter Ansatzpunkt zur methodischen Verbesserung agrarökonomischer Wirkungsanalysen ist die stärkere Berücksichtigung der Dynamik von Entwicklungen. In der empirischen Analyse zeigt sich, dass der Erklärungsgehalt von dynamischen Modellen immer höher war als der von statischen Modellen und dass der Effekt der Programmteilnahme in den statischen Modellen deutlich überschätzt wurde. Allerdings wurden in dieser Arbeit Paneldaten mit sechs Untersuchungsjahren verwendet - ein zu kurzer Zeitraum um die Vorteile der derzeit verfügbaren dynamischen Modelle (z. B. GMM-Modelle) vollständig nutzen zu können. Generell scheint die Diskussion zur Effizienz und Konsistenz dynamischer Panelschätzer noch nicht abgeschlossen zu sein (vgl. z. B. NEWEY und WindMEIJER, 2009), so dass auch hier neue Entwicklungen zu erwarten sind. 
Die Nettowirkungen direkter Transferzahlungen können aufgrund von Verdrängungs- und Substitutionseffekten in der Regel nicht einzelbetrieblich ermittelt werden. Idealerweise sollten daher mikroökonomische Analysen durch makroökonomische Untersuchungsansätze komplementiert werden. Ein methodisch anspruchsvollerer Ansatz ist die Verbindung der mikro- und makroökonomischen Analyse in einem strukturellen (Mehrebenen-) Modell (SINGER und WiLleTt, 2003). Entsprechende Anwendungsbeispiele aus der Agrarökonomie liegen nach Kenntnisstand der Autorin bisher nicht vor. Ein weiterer methodischer Ansatzpunkt zur mikro- und makroökonomischen Analyse von Maßnahmen der „Zweiten Säule“ der GAP ist deren Integration in bestehende regionale Sektormodelle wie RAUMIS oder CAPRI (BRITZ und HECKELEI, 2008).

Neue Methoden werden sich in der (agrarökonomischen) Evaluationspraxis nur durchsetzen, wenn diese mit Hilfe statistischer Standardsoftware implementiert werden können. Ein Beispiel hierfür sind die von LEUVEN und SIANESI (2003) und BECKER und ICHINO (2002) entwickelten Matching-Programme für Stata. Diese haben zu einer vermehrten Anwendung des Matching-Ansatzes geführt, berücksichtigen derzeit aber ausschließlich binär skalierte Politikvariablen. Neue Matching-Methoden für diskret oder stetig skalierte Politikvariablen können für die empirische Analyse unter Umständen besser geeignet sein, sind jedoch für empirisch arbeitende Forscher - ohne unterstützende Software - derzeit nicht zugänglich.

\section{Daten}

Die Effekte von Agrarumweltmaßnahmen und der Ausgleichszulage dürften maßgeblich vom Umfang der geförderten Fläche, von der Prämienhöhe je Hektar und von der geförderten Agrarumweltmaßnahme (Grünlandextensivierung etc.) abhängen. Diese Informationen sind in den verwendeten Buchführungsdaten bzw. im Testbetriebsnetz des BMELV nicht enthalten. Auch für die Evaluation der Agrarinvestitionsförderung fehlen in den Testbetriebsdaten die entsprechenden Informationen (Höhe und Art der Förderung). Wirkungsanalysen einzelbetrieblich orientierter Maßnahmen der „Zweiten Säule“ der GAP sind daher bisher nicht bzw. nur mit starken Einschränkungen auf Basis der Testbetriebsdaten möglich. ${ }^{76}$ Zukünftig werden (vermutlich) gerade diese Maßnahmen weiter an Bedeutung zunehmen. Die Aufnahme zusätzlicher Variablen in das Testbetriebsnetz des BMELV bzw. die FADN-Daten ist daher eine Voraussetzung, um diese Maßnahmen zukünftig einer belastbaren Wirkungsanalyse unterziehen zu können.

76

Die Nutzung alternativer Datenquellen ist mit einem sehr hohen Aufwand verbunden (z. B. Auflagenbuchführung für das AFP) bzw. nicht möglich, da z. B. die Daten des Integrierten Verwaltungs- und Kontrollsystems (InVeKos) keine betriebswirtschaftlichen Informationen enthalten. 


\section{Empirie}

Ein Großteil der empirischen ex-post Analysen zu den Wirkungen von direkten Transferzahlungen stammt aus den USA. Deutlich weniger ex-post Untersuchungen liegen für Europa vor und hier schwerpunktmäßig für die finanziell (bisher) weniger bedeutsamen Maßnahmen der „Zweiter Säule“. In Europa besteht ein erheblicher Bedarf, die agrarstrukturellen und produktionsrelevanten Wirkungen von verschiedenen Transferzahlungen (einheitliche Flächenprämie, Agrarinvestitionsmaßnahmen, Agrarumweltmaßnahmen, Ausgleichszulage) $\mathrm{zu}$ analysieren. Hierauf hat bereits die OECD (2006) verwiesen. Im Rahmen der „Zweiten Säule“ der GAP werden darüber hinaus seit vielen Jahren Maßnahmen gefördert, deren Wirkungen auf den Agrarsektor bis heute unklar sind (z. B. Förderung der Verarbeitung und Vermarktung, Diversifizierung, Beratung). Auch mit Blick auf den Ausbau der „Zweiten Säule“ sind für diese Maßnahmen Wirkungsanalysen notwendig.

In der empirischen Analyse wurde gezeigt, dass die Höhe der Effekte nicht nur vom Programm selbst, sondern vor allem von betrieblichen und regionalen Merkmalen abhängen. Neben der Schätzung durchschnittlicher Effekte, sollte daher auch die Variabilität der Wirkungen in Abhängigkeit von typischen Merkmalen (Betriebsgröße, Betriebsform, Region etc.) untersucht werden.

\section{Politikevaluation}

Der Rahmen der verpflichtend durchzuführenden Evaluation von Maßnahmen der „Zweiten Säule" der GAP wird durch die Gemeinsamen Bewertungsfragen der EU-KOM gesetzt. Ein inhaltlicher Schwachpunkt dieses Evaluationsrahmens ist die ausschließliche Orientierung der Bewertungsfragen auf die (zahlreichen) politischen Ziele der Maßnahmen. Dies hat zwei Nachteile: Zum einen werden nicht intendierte, gesamtwirtschaftlich aber durchaus relevante Wirkungen auf die Produktion oder die Agrarstruktur nicht erfasst. Zum anderen wird viel Zeit für die Erhebung von Nebensächlichkeiten verwendet - zu Lasten der Analyse der eigentlich relevanten Wirkungen und Kosten. Die Kosten der Politik werden sogar nur teilweise erfasst: Die Analyse der Transaktionskosten ist in dem bisherigen Bewertungsschema nicht vorgesehen. Eine für die Politikbewertung notwendige Betrachtung der Kosten-Wirksamkeit von Maßnahmen ist daher nicht möglich. Um den Nutzen von Evaluationen für die zukünftige Politikgestaltung zu erhöhen, bedarf es einer Konzentration der Evaluationsaktivitäten auf die jeweils relevanten Fragen. 


\section{Literaturverzeichnis}

ABbring JH, V. D. BERG G (2003) The nonparametric identification of treatment effects in duration models. Econometrica 71, 1491-1517

Adams G, Westhoff P, Willott B, Young II RE (2001) Do "decoupled" payments affect U.S. crop area? Preliminary evidence from 1997-2000. American Journal of Agricultural Economics 83 (5), 1190-1195

Agra CEAS CONSUlting (2003) Ex-post evaluation of measures under regulation (EC) No. 950/97. EU-Kom, DG Agri:

http://europa.eu.int/comm/agriculture/eval/reports/950/index_en.htm.

Stand 11.4.2006

Ahearn MC, El-Osta H, Dewbre J (2006) The impact of coupled and decoupled government subsidies on off-farm labor participation of U.S. farm operators. American Journal of Agricultural Economics 88 (2), 393-408

Ahearn MC, Yee J, HufFMan WE (2002) The impact of government policies on agricultural productivity and structure: Preliminary results. Research in Agricultural and Applied Economics: http://agecon.lib.umn.edu/. Stand 26.7.2007

AHEARn MC, YeE J, Korb P (2005) Effects of differing farm policies on farm structure and dynamics. American Journal of Agricultural Economics 87 (5), 1182-1189

ANDERSON TW, HSIAO C (1982) Formulation and estimation of dynamic models using panel data. Journal of Econometrics 18, 47-82

ANDERSON TW, SøRENSEN BE (1996) GMM estimation of a stochastic volatility model: a Monte Carlo study. Journal of Business \& Economic Statistics 14, 328-352

ANDERSSON FCA (2004) Decoupling: The concept and past experiences. SLI Working Paper 1. Swedish Institute for Food and Agricultural Economics: www.sli.lu.se. Stand 7.9.2009

ANGRIST JD (1999) Estimation of limited dependent variable models with dummy endogenous regressors: Simple strategies of empirical practice. Journal of Business \& Economic Statistics 29 (1), 2-15

Arellano M, Bond S (1991) Some tests of specification for panel data: Monte Carlo evidence and an application to employment equations. Review of Economic Studies 58, 277-297

Arellano M, Bover O (1995) Another look at the instrumental variables estimation of error-components models. Journal of Econometrics 68, 29-51

ART Forschungsgruppe AgRar und REgionalentwicklung TRIESDORF (2005) Aktualisierung der Halbzeitbewertung des Plans zur Entwicklung des ländlichen Raumes in Bayern. Im Auftrag des Bayerischen Staatsministeriums für Landwirtschaft und Forsten 
BAA, Bundesagentur FÜR Arbeit (2005) Arbeitslose nach Kreisen. März 2005. Bundesagentur für Arbeit:

http://www.pub.arbeitsamt.de/hst/services/statistik/detail/a.html.

Stand 8.6.2005

BALKHAUSEN O (2007) Effects of decoupling direct payments on agricultural production and land use in individual member states of the European Union. Dissertation (Georg-August-Universität Göttingen, Fakultät für Agrarwissenschaften)

BASER O (2006) Too much ado over propensity score models? Comparing methods of propensity score matching. Value in Health 9 (6), 377-385

BAUER DJ, CURRAN PJ (2005) Probing interactions in fixed and multilevel regression: Inferential and graphical techniques. Multivariate Behavioral Research 40 (3), $373-400$

BAum CF, Schaffer ME, Stillmann S (2003) Enhanced routines for instrumental variables/GMM estimation and testing. Boston College Economics Working Paper 667

BAUR PT (1997) Agrarstrukturwandel in der Schweiz. Dissertation (ETH Zürich)

BBR, BUNDESAMT FÜR BAUWESEN UND RAUMORDNUNG (2003) INKAR, Indikatoren und Karten zur Raumentwicklung. Aktuelle Daten zur Entwicklung der Städte, Kreise und Gemeinden. CD-Rom

Beck M, Dogot T (2006) The use of impact indicators for the evaluation of farm investment support - a case study on the Rural Development Programme for Wallonia (2000-2006). In: Bergschmidt A, DirksMeyer W, EFKEN J, Forstner B, Uetrecht I (ed.) Proceedings of the European Workshop on the Evaluation Farm Investment Support, Investment Support for Improvement of Processing and Marketing of Agricultural Products. Arbeitsberichte des Bereichs Agrarökonomie 03/2006, 69-77

BECKER SO, ICHINO A (2002) Estimation of average treatment effects based on propensity scores. Stata Journal 2 (4), 358-377

Bergschmidt A, Dirksmeyer W, Efken J, Forstner B, Uetrecht I (2006) Proceedings of the European Workshop on the Evaluation of Farm Investment Support, Investment Support for Improvement of Processing and Marketing of Agricultural Products. Arbeitsberichte des Bereichs Agrarökonomie 03/2006.

Bernhards U, Doll H, Klockenbring C, Plankl R, Rudow K (2003) Zwischenbewertung der Ausgleichszulage in benachteiligten Gebieten 2000 bis 2002. Länderübergreifender Bericht. Institut für Betriebswirtschaft, Agrarstruktur und Ländliche Räume (FAL)

BFNL, Bundesforschungsanstalt FÜR NAtURsChutZ Und LANDESCHAFTSÖKOlOGIE (1993) LANIS, Landschaftsinformationssystem. Gebietskulisse der Benachteiligten Gebiete in Deutschland. Stand: 1992 
Bichler B, LipPert C, HÄRING A, DABBert S (2005) Die Bestimmungsgründe der räumlichen Verteilung des ökologischen Landbaus in Deutschland. Berichte über Landwirtschaft 83 (1), 50-75

BLUNDELL R, BOND S (1998) Initial conditions and moment restrictions in dynamic panel data models. Journal of Econometrics 87, 115-143

BLUNDELl R, BOND S (2000) GMM estimation with persistent panel data: An application to production functions. Econometric Reviews 19, 312-340

BMELV, BUNDESMINISTERIUM FÜR ERNÄHRUNG, LANDWIRTSCHAFT UND VERBRAUCHERSCHUTZ (2006a) Buchführung der Testbetriebe. Ausführungsanweisung zum BMELV-Jahresabschluss. BMELV: www.bmelv.de. Stand 15.9.2007

BMELV, BUNDESMINISTERIUM FÜR ERNÄHRUNG, LANDWIRTSCHAFT UND VERBRAUCHERSCHUTZ (2006b) Bundestabelle. Stand: April 2006. Unveröffentlicht

BMVEL, BUNDESMINISTERIUM FÜR VERBRAUCHERSCHUTZ, ERNÄHRUNG UND LANDWIRTSCHAFT (2000) GAK-Rahmenplan 2000-2004. BMVEL:

http://bmvel.zadi.de.gak/. Stand: 11.4.2006

BMVEL, BUNDESMINISTERIUM FÜR VERBRAUCHERSCHUTZ, ERNÄHRUNG UND LANDWIRTSCHAFT (2005) Agrarbericht 2005 der Bundesregierung

Bond S (2002) Dynamic panel data models: A guide to micro data methods and practice. cemmap working paper CWP09/02

BRAND-SAßEN H (2009) Landwirtschaftliche Rentenbank, Telefonische Mitteilung am 9.9.2009

BRITZ W, HECKELEI T (2008) Recent developments in EU policies - challenges for partial equilibrium models. In: BARTOVA L, M'BAREK R, RATINGER T (Hrsg.) Modelling agricultural and rural development policies: Proceedings, 107th EAAE Seminar, 29th January - 1st February, 2008, Seville

BROTHERTON I (1991) What limits participation in ESAs? Journal of Environmental Management 32, 241-249.

BRÜMMER B, LOY JP (2000) The technical efficieny impact of farm credit programs: A case study of Northern Germany. Journal of Agricultural Economics 51 (3), 405-418

BURfisher ME, Hopkins J (Hrsg.) (2003) Decoupled payments. Household income transfers in contemporary U.S. Agriculture

Burgath A, Doll H, Fasterding F, Grenzebach M, Klare K, Plankl R, WARNEBOLDT S (2001) Ex-post-Evaluation von Maßnahmen im Rahmen der Verordnung (EG) Nr. 950/97 für den Förderzeitraum 1994 bis 1999 in Deutschland, Endbericht

CAHILL SA (1997) Calculating the rate of decoupling for crops under CAP oilseeds reform. Journal of Agricultural Economics 48(3), 349-378 
CAHILL C (2004) Risk effects of PSE crop measures. Regional meeting on agriculture trade and development in Southeast Asian Countries, OECD

CALIEndo M (2006) Microeconometric Evaluation of Labour Market Policies. Lecture Notes in Economics and Mathematical Systems. Springer

Caliendo M, Kopeinig S (2008) Some Practical Guidance for the Implementation of Propensity Score Matching. Journal of Economic Surveys 22(2), 31-72

Cameron AC, Trivedi PK (2005) Microeconometrics. Methods and applications. Cambridge University Press

Chamberlain G (1982) Multivariate regression models for panel data. Journal of Econometrics 18, 5-46

Chamberlain G (1992) Comment: Sequential moment restrictions in panel data. Journal of Business \& Economic Statistics 10, 20-26

Chambers RG, Foster WE (1983) Participation in the farmer-owned reserve program: a discrete choice model. American Journal of Agricultural Economics 65 (1), 120-124.

Chambers RG, Just, DR (1989) Estimating multi-output technologies. American Journal of Agricultural Economics 71, 980-995

Chang HH, Boisvert RN (2005) Are farmers' decisions to work off the farm and participate in conservation reserve program independent, joint or sequential? Research in Agricultural and Applied Economics: http://agecon.lib.umn.edu/. Stand 26.7.2007

Chang HH, Lambert DM, Mishra AK (2008) Does participation in the conservation reserve program impact the economic well-being of farm households? Agricultural Economics 38 (2), 201-212

Christensen B, Clement M, Albers S, Guldner S (2005) Zur Relevanz der Kontrollgruppenauswahl in der empirischen Forschung. http://www.uni-kiel.de/ifw/staff/christensen.htm. Stand: 8.8.2007

COCHRAN W, Rubin DB (1973) Controlling bias in observational studies. Sankyha: The Indian Journal of Statistics 35, 417-446

COHEN J (1988) Statistical power analysis for the behavioral science (2. Ausgabe)

COOK TD, SHADISH WR, Wong VC (2007) Three conditions under which experiments and observational studies often produce comparable causal estimates: New findings from within-study comparisons. Working Paper. In: Contributions to the Symposium on Causality 2008 "Causal effects - Design and Analysis", July $17^{\text {th }}$ to $19^{\text {th }}, 2008$ in Dornburg, Jena

Crabtree B, Chalmers N, BARron N-J. (1998) Information for policy design: Modelling the participation in a farm woodland incentive scheme. Journal of Agricultural Economics 49 (3), 306-320 
Defrancesco E, Gatto P, Runge F, Trestini S (2008) Factors affecting farmers' participation in agri-environmental measures: A northern Italian perspective. Journal of Agricultural Economics 59 (1), 114-131

DenejIA R, WahBA S (2002) Propensity score matching methods for nonexperimental causal studies. Review of Economics and Statistics 84 (1), 151-161

Destatis, Statistisches Bundesamt (div. Jgg.) Land- und Forstwirtschaft, Fischerei. Betriebsgrößenstruktur. Fachserie 3, Reihe 2.1.1

DLG, Deutsche Landwirtschaftsgesellschaft (2006) Effiziente Jahresabschlussanalyse. Arbeiten der DLG 194

Doluschitz R, Forstner B, Friemel G, Grosskopf W, Kappelmann KH, Lenz R, Plankl R, TSCHMARKe A, WendT H (2006) Up-date der Halbzeitbewertung des EPLR Baden-Württemberg 2000-2004. Maßnahmen- und Entwicklungsplan Ländlicher Raum - EPLR Baden-Württemberg 2000-2006. Im Auftrag des Landes Baden-Württemberg. Ministerium für Ernährung und den Ländlichen Raum: http://www.landwirtschaft-mlr.badenwuerttemberg.de/servlet/PB/menu/ 1040914/index.html. Stand 22.6.2006

El-Osta H, Mishra AK, AHEARn MC (2004) Labor supply by farm operators under "decoupled" farm program payments. Review of Economics of Households 2 (4), 367-385

El-Osta H, Mishra AK, Morehart MJ (2008) Off-Farm Labor Participation Decisions of Married Farm Couples and the Role of Government Payments. Review of Agricultural Economics 30 (2), 311-332

Engel S, PAgiola S, Wunder S (2008) Designing payments for environmental services in theory and practice: An overview of the issues. Ecological Economics 65, 663674

ESPOSTI R (2007) Regional Growth and policies in the European Union: Does the common agricultural policy have a counter-treatment effect? American Journal of Agricultural Economics 89 (1), 116-134

EU-KOM, EUROPÄISCHE KOMMISSION (2000) Katalog gemeinsamer Bewertungsfragen mit Kriterien und Indikatoren. Dokument VI/12004/00 endg. (Teil B)

EU-KOM, EUROPÄISCHE KOMMISSION (2006a) Common Monitoring and Evaluation Framework (CMEF)

EU-KOM, EuropÄISCHE Kommission (2006b) EU-Budget 2005. Europäische Kommission, DF Finanzplanung und Haushalt:

http://eur.lex.europa.eu/budget/www/index-en.htm. Stand: 10.1.2008

EU-KOM, EUROPÄISCHE KOMMISSION, Generaldirektion Landwirtschaft (2006c) 35. Finanzbericht über den Europäischen Ausrichtungs- und Garantiefonds für die Landwirtschaft, Abteilung Garantie. Haushaltsjahr 2005. KOM(2006) 512 endgültig 
EUROPÄISCHER RECHNUNGSHOF (2003) Sonderbericht zur Ländlichen Entwicklung: Förderung der benachteiligten Gebiete. Kurzinformationen 4/2003

EUROSTAT (2006) Einkaufspreise landwirtschaftlicher Betriebsmittel (absolute Preise) jährlicher Preis:

http://epp.eurostat.cec.eu.int/portal/page/portal/eurostat/home.

Stand 26.8.2009

EvANS DS (1987) Empirical analysis of size distribution of farms: Discussion. American Journal of Agricultural Economics 69, 484-485

EVENSON RE, HUFFMAN WE (1997) Long-run structural and productivity change in U.S. agriculture: Effects of prices and policies. Research in Agricultural and Applied Economics: http://agecon.lib.umn.edu/. Stand: 26.7.2007

FAL, BUNDESFORSCHUNGSANSTALT FÜR LANDWIRTSCHAFT INSTITUT FÜR LÄNDLICHE RÄUME, ARUM, ARBEITSGEMEINSCHAFT UMWELT- UND STADTPLANUNG UND BFH, BundesAnstalt FÜR HolzForsChung (2006) Aktualisierung der Halbzeitbewertung der Entwicklungspläne für den ländlichen Raum für die Bundesländer Bremen, Hamburg, Hessen, Niedersachsen, Nordrhein-Westfalen und Schleswig-Holstein. Institut für Ländliche Räume (FAL)

FÄHRMANN B, GRAJEWSKI R (2008): Implementationskosten des NRW-Programms Ländlicher Raum vor dem Hintergrund der erzielten Wirkungen. Materialband zu Kapitel 10. In: Ex-post-Bewertung des NRW-Programms Ländlicher Raum gem. Verordnung (EG) Nr. 1257/1999. Johann Heinrich von Thünen-Institut (vTI): http://www.vti.bund.de/fallitdok_extern/bitv/dk041379.pdf.

Stand 18.08.2009

FÄHRMANN B, GRAJEWSKI R (2008a): Implementationskosten von PROLAND NIEDERSACHSEN vor dem Hintergrund der erzielten Wirkungen. Materialband zu Kapitel 10. In: Ex-post-Bewertung von PROLAND NIEDERSACHSEN: Programm zur Entwicklung der Landwirtschaft und des ländlichen Raumes gem. Verordnung (EG) Nr. 1257/1999. Johann Heinrich von Thünen-Institut (vTI)

FÄHRMANN B, GRAJEWSKI R (2008b): Implementationskosten des Programms "Zukunft auf dem Land" (ZAL) vor dem Hintergrund der erzielten Wirkungen. Materialband zu Kapitel 10. In: Ex-post-Bewertung des Programms "Zukunft auf dem Land" (ZAL) gem. Verordnung (EG) Nr. 1257/1999. Johann Heinrich von ThünenInstitut (vTI)

FÄHRMANN B, GRAJEWSKI R (2008c): Implementationskosten des hessischen Entwicklungsplans für den ländlichen Raum vor dem Hintergrund der erzielten Wirkungen. Materialband zu Kapitel 10. In: Ex-post-Bewertung des hessischen Entwicklungsplans für den ländlichen Raum gem. Verordnung (EG) Nr. 1257/1999. Johann Heinrich von Thünen-Institut (vTI)

FISHER RA (1951) The design of experiments. 2. Ausgabe 
FreistaAt ThÜRINGEN (2008) Entwicklungsplan für den ländlichen Raum 2000-2006. Lagebericht für das Jahr 2005. EAGFL - Abteilung Garantie. Unveröffentlicht

GAK-Berichterstattung (div. Jgg.) Die Verbesserung der Agrarstruktur in der Bundesrepublik Deutschland. Bericht des Bundes und der Länder über den Vollzug der Gemeinschaftsaufgabe "Verbesserung der Agrarstruktur und des Küstenschutzes"

GALE HF (1994) Longitudinal analysis of farm size over the farmers life cycle. Review of Agricultural Economics 16, 11-23

Gasson R, Crow G, Errington A, Hutson J, Marsden T, Winter DM (1995) The farm as a family business: A review. In: Peter GH (Hrsg.) Agricultural economics. International Library of critical writings in economics, 3-43

Girante MJ, Goodwin BK, Featherstone AM (2008) Farmers' crop acreage decision in the presence of credit contraints: Do decoupled payments matter? Selected paper prepared for presentation at the American Agricultural Economics Association annual meeting, Orlando, FL, July, 27-29, 2008. Research in Agricultural and Applied Economics: http://agecon.lib.umn.edu/. Stand 26.4.2009

Glauben T, Tietje H, Weiss CR (2005) Agriculture on the move. Exploring regional difference in farm exit rates in Western Germany. Jahrbuch für Regionalwissenschaft $26,103-118$

Godtland EM, Sadoulet E, De Janvry A, Murgai R, Ortiz O (2004) The Impact of Farmer Field Schools on Knowledge and Productivity: A Study of Potato Farmers in the Peruvian Andes". Economic Development and Cultural Change $53,63-92$

Goetz SJ, Debertin DL (2001) Why farmers quit. A county-level analysis. American Journal of Agricultural Economics 83 (4), 1010-1023

Gohin A, Guyomard H, Le MouËL C (2000) Measuring the degree of decoupling of alternativ internal support policy instruments: The green box issue. Research in Agricultural and Applied Economis: http://agecon.lib.umn.edu/. Stand 7.9.2009

GOODWIN BK, Mishra AK (2002) Are 'decoupled' farm program payments really decoupled? An empirical evaluation. mimeo, Ohio State University:

http://departments.agi.huij.ac.il/economics/kenes-goodwin2.pdf.

Stand: 2.5.2007

GoODwIN BK, Mishra AK (2004) Farming efficiency and the determinants of multiple job holding by farm operators. American Journal of Agricultural Economics $86(3), 722-729$

Goodwin BK, Mishra AK, Kimhi A (2007) Policy interactions at the farm level: An evaluation of participation in the conservation reserve program and related policy decisions. Research in Agricultural and Applied Economics: http://agecon.lib.umn.edu/. Stand 26.7.2007 
GREENE WH (2003) Econometric Analysis

GU XS, Rosenbaum PR (1993): Comparison of multivariate matching methods: Structures, distances and algorithms. Journal of Computation and Graphical Statistics 2 (4), 405-420

Guyomard H, Le MouËL C, GoHin A (2004) Impact of alternative agricultural income support schemes on multiple policy goals. European Review of Agricultural Economics 31 (2), 125-148

HADRI K (2000) Testing for stationarity in heterogeneous panel data. Econometrics Journal 3, 148-161

Hallam A (1993) Empirical studies of size, structure and efficiency in agriculture. In: BOUlDER et al. (Hrsg.) Size, structure and the changing face of American agriculture, 204-231

HANSEN BB (2004) Full matching in an observational study of coaching for the SAT. Journal of the American Statistical Association 99 (467), 609-618

HANSEN LP (1982) Large sample properties of generalised method of moments estimators. Econometrica 50, 1029-1054

HARRINGTON DH, REINSEL RD (1995) A synthesis of forces driving structural change. Canadian journal of agricultural economics, Special issue 1995 (43), 3-14

HECKMAN J (1976) The common structure of statistical models of truncation, sample selection, and limited dependent variables and a simple estimator for such models. Annals of Economic and Social Measurement 5/4, 475-492

Heckman J (1978) Dummy Endogenous Variables in a Simultaneous Equation System. Econometrica 47 (4), 153-161

Heckman J (1990) Varieties of Selection Bias. The American Economic Review 80 (2), 313-318

HECKMAN J (1992) Randomization and social policy evaluation. IN: MANSKI CF, GARFINKEL I. (Hrsg.) Evaluating welfare and training programs, 201-230

Heckman J, Hotz J, Holland PW, Moffitt R (1989) Choosing among alternative nonexperimental methods for estimating the impact of social programs: The case of manpower training. Journal of the American Statistical Association 84 (408), 862-874

HECKMAN J, ICHIMURA H, TODD PE (1997) Matching as an econometric evaluation estimator: evidence from evaluating a job training program. Review of Economic Studies 64 (4), 605-654

HECKMAN J, ICHIMURA H, TODD PE (1998) Matching as an econometric evaluation estimator. Review of Economic Studies 65, 261-294 
Heckman J, LaLonde RJ, Smith, J (1999) The economics and econometrics of active labor market programs. In: AsHENFELTER O, CARD DE (Hrsg.) Handbook of Labor Economics. Vol. III, 1865-2097

HECKMAN J, NAVARRO-LOZANO S (2004) Using matching, instrumental variables and control functions to estimate economic choice models. Review of Economics and Statistics $86(1), 30-57$

Heckman J, Navarro S (2007) Dynamic discrete choice and dynamic treatment effects. Journal of Econometrics 136 (2), 341-396

Heckman J, RobB R (1985) Alternative Methods for Evaluating the Impact of Interventions. In: HECKMAN J., SINGER B (Hrsg.) Longitudinal Analysis of Labor Market Data, 156-245

HeckMan J, UrzÚA S (2008) Comparing IV with structural models: What simple IV can and cannot identify. IZA Discussion Paper No. 3980

Heckman J, Vytlacil EJ (2007a) Econometric evaluation of social programs, part I: Causal models, structural models and econometric policy evaluation. In: HeCKMAN JJ, Leamer E (Hrsg.) Handbook of Econometrics. Vol. VIb, 47794874

HeCKMAn J, VytLacil EJ (2007b) Econometric evaluation of social programs, part II: Using the marginal treatment effect to organize alternative econometric estimators to evaluate social programs, and to forecast their effects in new environments. In: HECKMAN JJ, LEAMER E (Hrsg.) Handbook of Econometrics, Vol. VIb, 4675-5143

HENNESSY DA (1998) The production effects of agricultural income support policies under uncertainty. American Journal of Agricultural Economics 80 (1), 46-57

HENNING HCA (1994): Unternehmens-Haushalts-Modelle. Eine theoretische und empirische Analyse

HenNing HCA, MichaleK J (2008a) Theoretische Grundlagen und die Rolle parametrischer und nicht-parametrischer Modellansätze in der Politikevaluation. Replik zu Anne Margarian. Agrarwirtschaft 57 (7), 348-349

Henning HCA, MichaleK J (2008b) Ökonometrische Methoden der Politikevaluation: Meilensteine für eine sinnvolle Agrarpolitik der 2. Säule oder akademische Fingerübung. Agrarwirtschaft 57 (3/4), 232-243

Henrichsmeyer W, Witzke HP (1994) Agrarpolitik. Band 2: Bewertung und Willensbildung

HOFER F (2002) Strukturwirkung von Direktzahlungen. Dissertation (ETH Zürich)

Holland PW (1986) Statistics and causal inference. Journal of American Statistical Association 81 (396), 945-960 
Holtz-Eakin D, Newey W, Rosen HS (1988) Estimating vector autoregressions with panel data. Econometrica 56, 1371-1396

Hovorka G (2006) The influence of agricultural policy on the structure of mountain farms in Austria. Contribution to the 96th EAAE-Seminar "Causes and Impacts of Agricultural Structures", January 2006, Taenikon, Switzerland. Forschungsanstalt Agroscope Reckenholz-Tänikon (ART):

http://www.fat.admin.ch/eaae96/abstracts/s49.pdf. Stand 26.5.2007

HsiaO C (2003) Analysis of panel data

HufFMAN WE (1991) Agricultural Household Models: Survey and Critique. In: Hallberg MC, Findeis J, Lass D (Hrsg.) Multiple job-holding among farm families, 79-111

HuFFMAN WE, EvENSON RE (2001) Structural and productivity change in U.S. agriculture 1950-1982. Agricultural Economics 24 (2), 127-147

HUFFMAN WE, LANGE MD (1989) Off-farm work decision of husbands and wives: Joint decision making. Review of Economics and Statistics LXXI (3), 471-480

HyNes S, GARVEY E (2009) Modelling farmers' participation in an agri-environmental scheme using panel data: An application to the rural environment protection scheme. Journal of Agricultural Economics 60 (3), 546-562

IEEP, Institute for European Environmental Policy (2006) An evaluation of the Less Favoured Area measure in the 25 member states of the European Union. European Commission, DG Agri:

http://ec.europa.eu/agriculture/eval/reports/lfa. Stand 7.7.2007

IFLS, Institut für Ländliche Strukturforschung (2005) Aktualisierung der Halbzeitbewertung des rheinland-pfälzischen Entwicklungsplans "Zukunftsinitiative für den ländlichen Raum" (ZIL) im Zeitraum 2000-2006. Im Auftrag des Ministeriums für Wirtschaft, Verkehr, Landwirtschaft und Weinbau (MWVLW) des Landes Rheinland-Pfalz

IMBENS GW (2000) The role of the propensity score estimating dose-response function. Biometrica 87 (3), 706-710

IMBENS GW, ANGRIST JD (1994) Identification and estimation of Local Average Treatment Effects. Econometrica 62, 467-476

JovANOVIC B (1982) Selection and evolution of industry. Econometrica 50 (3), 649-670

Key N, Lubowski RN, ROBERTS MJ (2005) Farm-level production effects from participation in government commodity programs: Did the 1996 federal agricultural improvement and reform act make a difference? American Journal of Agricultural Economics 87 (5), 1211-1219 
Key N, RoBerts MJ (2003) Government payments and structural change in agriculture. Research in Agricultural and Applied Economics: http://agecon.lib.umn.edu/. Stand 26.7.2007

Key N, Roberts MJ (2006) Government payments and farm business survival. American Journal of Agricultural Economics 88 (2), 382-392

Kim CS, Schluter G, Schaible G, Mishra AK, Hallahan C (2005) A decomposed negative binominal model of structural change: A theoretical and empirical application to U.S. agriculture. Canadian Journal of Agricultural Economics 53 (June), 161-176

Kleinhanß W, Bertelsmeier M, Manegold D, Offermann F, Osterburg B, SALAMON P (2003) Folgenabschätzung der Legislativvorschläge zur Halbzeitbewertung der Agenda 2000. Institut für Betriebswirtschaft, Agrarstruktur und Ländliche Räume, Institut für Marktanalyse und Agrarhandelspolitik (FAL): http://www.bal.fal.de/download/ab02-2004.pdf. Stand 1.4.2004

KOESTER U (1992) Grundzüge der landwirtschaftlichen Marktlehre

KUHLMANN D (1997) Varianten direkter Einkommensübertragungen in der Landwirtschaft. Verwaltungsaufwand und einzelbetriebliche Wirkungen. Dissertation (Georg-August-Universität Göttingen)

Kwiatowski D, Phillips PCB, Schmidt P, Shin Y (1992) Testing the null hypothesis of stationarity against the alternative of a unit root. How sure are we that economic time series have a unit root? Journal of Econometrics 54, 159-178

KYRIAZIDOU E (2001) Estimation of dynamic panel data sample selection models. Review of Economic Studies 68 (3), 543-572

LAMBERT DM, GRIFFIN TW (2004) Analysis of government farm subsidies on farmland cash rental rates using a fixed effect spatial distributed lag model and a translog cost model. Research in Agricultural and Applied Economics: http://agecon.lib.umn.edu/. Stand 26.7.2007

LAMbert DM, Schaible G, JohAnSSON R, DABERKow S (2006) Working-Land conservation structures: Evidence from program and non-program participants. Research in Agricultural and Applied Economics: http://agecon.lib.umn.edu/. Stand 26.7.2007

LAND Use Policy Group (2008) An analysis of the potential effectiveness of a payment-by-results approach to the delivery of environmental public goods and services supplied by agri-environment schemes. Final Report Project No: 23192. www.lupg.org.uk. Stand 29.8.2009

LAND-DATA GMBH (2006) Buchführungsdaten landwirtschaftlicher Betriebe für die Wirtschaftsjahre 1999/2000 bis 2004/2005. 
LANDGESELlSCHAFT SACHSEN-ANHALt mBH (2005a) Aktualisierung der Halbzeitbewertung des EPLR Sachsen-Anhalt. Im Auftrag des Ministeriums für Landwirtschaft und Umwelt (MLU) des Landes Sachsen-Anhalt: www.mlu.sachsenanhalt.de. Stand 22.6.2006

LANDGESEllschaft SaChSEN-AnHalt mbH (2005b) Aktualisierung der Halbzeitbewertung des EPLR Sachsen. Im Auftrag des Sächsischen Ministeriums für Umwelt und Landwirtschaft (SMUL):

http://www.smul.sachsen.de/de/wu/aktuell/foerderung/index_819.html.

Stand 22.6.2006

LATACZ-LOHMANN U, SCHILIZzI S (2007) Quantifying the benefits of conservation auctions. EuroChoices 6(3), 32-39

LEATHERS H (1992) Self-selection as a problem in evaluation agri-environmental programs. Paper presented at the $87^{\text {th }}$ EAAE-Seminar: Assessing rural development of the CAP. American Journal of Agricultural Economics 74 (2), 291-298

LECHNER M (2000) A note on the common support problem in applied evaluation studies. Discussion Paper, H. 2001-01. Swiss Institute for International Economics and Applied Economic Research (SIAW), University of St. Gallen. Stand 26.10.2006

LECHNER M (2002) Program heterogeneity and propensity score matching: An application to the evaluation of active labor market policies. Review of Economics and Statistics 84 (2), 205-220

LECHNER M (2004) Sequential matching estimation of dynamic causal models. IZA Discussion Paper 1042

LECHNER M (2005) A note on endogenous control variables in evaluation studies. Discussion Paper, H. 2005-16. Swiss Institute for International Economics and Applied Economic Research (SIAW), University of St. Gallen. Stand 26.10.2008

LENCE SH, Mishra AK (2003) The impact of different farm programs on cash rents. American Journal of Agricultural Economics 85 (3), 753-761

LEUVEN E, SiANESI B (2003) PSMATCH2: Stata module to perform full Mahalanobis and propensity score matching, common support graphing, and covariate imbalance testing, Statistical Software Components S432001, Boston College Department of Economics, revised 13 Apr 2006. RePEc, Research Papers in Economics: http://ideas.repec.org/c/boc/bocode/s432001.html. Stand 4.12.2006

LUDWIG-MAYERHOFER, W. (2002): Likelihood-Verhältnis-Test. ILMES, Internetlexikon der Methoden der empirischen Sozialforschung: http://www.lrz-muenchen.de/ w wlm/ilm_17.thm. Stand 12.9.2007 
LYNCH L, GRAY W, GEOGHEGAN J (2007a) Are farmland preservation program easement restrictions capitalized into farmland prices? What can a propensity score matching analysis tell us? Review of Agricultural Economics 29 (3), 502-509

LYNCH L, GRAY W, GEOGHEGAN J (2007b) Recursive or non recursive agricultural household decision making. Review of Agricultural Economics 29 (3), 502-509

LYNCH L, XiANPING L (2007) Impact of Designated Preservation Areas on Rate of Preservation and Rate of Conversion: Preliminary Evidence. American Journal of Agricultural Economics 89 (5), 1205-1210

MANN S (2003) Bestimmungsgründe des landwirtschaftlichen Strukturwandels. Agrarforschung $10(1), 32-36$

MARGARIAN A (2007) Mehr-Ebenen-Modell in der Analyse agrarstruktureller Entwicklungen - Methodik und Implikationen. Agrarwirtschaft 56 (7), 340-353

Margarian A (2008) Diskussionsbeitrag zu: HCA Henning und Michalek J: Ökonometrische Methoden der Politikevaluation: Meilenstein für eine sinnvolle Agrarpolitik der 2. Säule oder akademische Fingerübung? Agrarwirtschaft 57 (2008), Heft 3/4. Agrarwirtschaft 57 (7), 346-347

Mehl P, Plankl R (2001) "Doppelte Politikverflechtung" als Bestimmungsfaktor der Agrarstruktur- und der Agrarumweltpolitik in der BR Deutschland. Agrarwirtschaft 50 (3), 173-177

Mishra AK, GoOdwIN BK (1997) Farm income variability and the supply of off farm labour. American Journal of Agricultural Economics 79, 880-887

Moro D, ScKoKAI P (1999) Modelling the CAP arable crop regime in Italy: Degree of decoupling and impact of Agenda 2000. Cahiers d'Economie et Sociologie Rurales 53, 50-73

N.N. (2005) Updatebewertung des Plans zur Förderung der Entwicklung des Ländlichen Raumes (EPLR). Im Auftrag des Umweltministeriums des Saarlandes

NAKAJIMA C (1986) Subjective Equilibrium Theory of the Farm. Developments in Agricultural Economics

NEANDER E (1992) Zur Zukunft der Ausgleichszulage. Agrarwirtschaft 41 (8/9), 221-222

NeWEy WK, WindMeIJER F (2009) Generalized method of moments with many weak moment conditions. Econometrica 77 (3), 687-719

NEYMAN JS (1923) On the application of probability theory to agricultural experiments. Essay on principles. Section 9. Roczniki Nauk Rolniczych X, 1-51 [Translated and republished in Statistical Science 5, 365-484] 
NiEBERG H (1996) Wirtschaftliche Folgen der Umstellung auf ökologischen Landbau empirische Ergebnisse von 107 Betrieben aus den Alten Bundesländern. In: NiEBERG H (Hrsg.) Ökologischer Landbau: Entwicklung, Wirtschaftlichkeit, Marktchancen und Umweltrelevanz. FAL-Tagung am 26. und 27. September 1996. Landbauforschung Völkenrode 175, 57-73

NiEBERG H, OfFERMANN F (2006) Einkommensvergleich zwischen ökologischen und konventionellen Betrieben. Agra-Europe, Sonderbeilage 47 (18), 1-10

Nieberg H, Offermann F, Zander K (2007) Organic Farms in a Changing Policy Environment: Impacts of Support Payments, EU-Enlargement and Luxembourg Reform. Organic Farming in Europe: Economics and Policy 13

Nieberg H, Offermann F, Zander K, JäGersberg P (2005) Further Development of Organic Farming Policy in Europe, with Particular Emphasis on EU Enlargement D12: Report on the farm level economic impacts of OPF and Agenda 2000 implementation. Institute of Rural Studies, University of Wales Aberystwyth: http://www.irs.aber.ac.uk/EUCEEOFP/eu-ceeofp/reports/index.html. Stand 24.5.2007

OECD, Organisation FOR ECONOMiC Co-OPERATION AND DeVElopment (2001a) Decoupling: a conceptual overview

OECD, ORganisation fOR ECONOMIC Co-OPERATION AND DeVElopment (2001b) Market effects of crop support measures

OECD, ORganisation FOR ECONOMIC CO-OPERATION AND DEVElopment (2006): Decoupling - policy implications. OECD: http://www.oecd.org/LongAbstract/0,2546,00_2649_33727_36298854_119829 _1_1_1,00.html. Stand 9.9.2009.

OfFERMANN F, Nieberg H (2001) Economic performance of organic farms in Europe. Economics and Policy 5

OfFERMANN F, NieberG H (2003) Wirtschaftlichkeit des ökologischen Landbaus im internationalen Vergleich. In: RAHMANN G, KÜHNE S (Hrsg.) Ressortforschung für den ökologischen Landbau 2004. Landbauforschung Völkenrode, Sonderheft 273, 101-104

Offermann F, Nieberg H, Hecht J (2008) Potential of differentiated payment levels based on standard cost approaches: A case study of selected rural development measures in Germany. The 82nd Annual Conference of the Agricultural Economics Society Royal Agricultural College 31st March to 2nd April 2008. http://ageconsearch.umn.edu/handle/36866. Stand 29.8.2009

OLTMER K (2001) Impacts of agricultural policy reform and land prices: A quantitative analysis of the literature. Paper prepared for presentation at the 2001 AAEA-CAES Annual Meeting. Research in Agricultural and Applied Economics: http://agecon.lib.umn.edu/. Stand 26.7.2007 
ORTNER KM (2005) Impacts of assistance for marketing of agricultural quality products in Austria. In: ORTNER KM (Hrsg.) Assessing rural development policies of the Common Agricultural Policies. Selection of papers from the $87^{\text {th }}$ EAAESeminar of the European Association of Agricultural Economics (EAAE), 281296

OSTERBURG B (2000) Impact of agri-environment programmes - the case of Germany (background paper). Poster Contribution at the XXIV IAAE Conference, Berlin 2000

Osterburg B (2005) Chapter 10. Assessing long-term impacts of agri-environmental measures in Germany. OECD (Hrsg) Evaluating agri-environmental policies. Design, practice and results

Osterburg B (2007) Analysen zur Düngeverordnungs-Novelle vom Januar 2006. In: OsTERBURG B und RUNGE T (Hrsg.) Maßnahmen zur Reduzierung von Stickstoffeinträgen in Gewässer - eine wasserschutzorientierte Landwirtschaft zur Umsetzung der Wasserrahmenrichtlinie. Landbauforschung Völkenrode, Sonderheft $307,267-302$

Osterburg B, Plankl R, Bernhards U, Klockenbring C, Rudow K, Becker H, Kreins P, Stegmann S (2003) Auswirkungen der Luxemburger Beschlüsse auf ländliche Räume, Agrarumweltmaßnahmen und die Ausgleichszulage. Arbeitsberichte des Bereichs Agrarökonomie 9/2003

Osterburg B, Stratmann U (2002) Die regionale Agrarumweltpolitik in Deutschland unter dem Einfluss der Förderangebote der Europäischen Union. Agrarwirtschaft 51 (5), 259-279

Osterburg B, Wilhelm J, Nieberg H (1997) Darstellung und Analyse der regionalen Inanspruchnahme von Agrarumweltmaßnahmen gemäß VO (EWG) 2078/92 in Deutschland. Arbeitsbericht der FAL 8/97

PARSON LS (2001) Reducing bias in a propensity score matched-pair sample using greedy matching techniques. Proceedings of the 26th Meeting of the SAS International User Group (SUGI 26):

www2.sas.com/proceedings/sugi26/p214-26.pdf. Stand 5.10.2006

Peerlings J, Poelman N (2004) Wildlife and landscape services production in Dutch dairy farming; jointness and transaction costs. European Review of Agricultural Economics 31 (4), 427-449

Peters W (1980) Untersuchung zur Ausgleichzulage. Berichte über Landwirtschaft $58(2), 212-247$. 
Pfefferli S (2006) Impact Analysis of Investment Support for Agricultural Buildings in Switzerland. In: Bergschmidt A, DirksmeYer W, EFKEn J, Forstner B, Uetrecht I (ed.) Proceedings of the European Workshop on the Evaluation Farm Investment Support, Investment Support for Improvement of Processing and Marketing of Agricultural Products. Arbeitsberichte des Bereichs Agrarökonomie 03/2006, 147-158

Pietola K, VÄRE M, LANSINK AO (2003) Timing and type of exit from farming: farmers' early retirement programmes in Finland. European Review of Agricultural Economics 30 (1), 99-116

Plankl R, Brand-Saßen H, Daub R, Doll H, Pohl C, Rudow K (2005) Aktualisierung der Halbzeitbewertung der Ausgleichszulage in benachteiligten Gebieten 2001 bis 2004. Länderübergreifender Bericht. Institut für Betriebswirtschaft, Agrarstruktur und Ländliche Räume (FAL)

Plankl R, Brand-Saßen H, Daub R, Doll H, Pohl C, Rudow K (2006) Aktualisierung der Halbzeitbewertung der Ausgleichszulage in benachteiligten Gebieten 2002 bis 2004. Länderübergreifender Bericht. Institut für Betriebswirtschaft, Agrarstruktur und Ländliche Räume (FAL)

Plankl R, Neander E (1994) Zur Wirksamkeit der Ausgleichszulage in benachteiligten Gebieten der Bundesrepublik Deutschland. Agrarwirtschaft 43 (6), 236-243

PoHL C (2008) Kapitel 5.1: Maßnahmen 211 und 212: Ausgleichszulage für naturbedingte Nachteile in Berggebieten / in sonstigen benachteiligten Gebieten. In: TIETZ A (Hrsg.) Ländliche Entwicklungsprogramme 2007 bis 2013 in Deutschland im Vergleich - Finanzen, Schwerpunkte, Maßnahmen. Landbauforschung Völkenrode, Sonderheft 315, 83-90

PUfAHL A, WeIss CR (2007) Evaluating the effects of farm programs: Results from propensity score matching. Department of Economics Working Paper Series 114. Vienna University of Economics and Business Administration: http://epub.wu-wien.ac.at/wp/. Stand 20.12.2007

PufAHL A, WeISS CR (2009) Evaluating the effects of farm programs: Results from propensity score matching. European Review of Agricultural Economics 36 (1), 79-101

Rambøll Management (2005) Update der Halbzeitbewertung des EPLR 2000-2006. Im Auftrag des Ministeriums für Ernährung, Landwirtschaft, Forsten und Fischerei des Landes Mecklenburg-Vorpommern

RAmos X (2005) Kernreg: Stata module for kernel regression (Nadaraya-Watson estimator). StataCorp.: www.stata.com. Stand 29.4.2007

REINOWSKI E (2006) Mikroökonomische Evaluation und das Selektionsproblem. Zeitschrift für Evaluation 2006 (2), 187-226 
Reiter K, Roggendorf W (2008) Kapitel 5.3: Maßnahmen 214: Zahlungen für Agrarumweltmaßnahmen. In: TIETZ A (Hrsg.) Ländliche Entwicklungsprogramme 2007 bis 2013 in Deutschland im Vergleich - Finanzen, Schwerpunkte, Maßnahmen. Landbauforschung Völkenrode, Sonderheft 315, 91-97

Reiter K, Roggendorf W, Pufahl A, Essmann S, Horlitz T, Sander A (2003a) Halbzeitbewertung des NRW-Programms ländlicher Raum gem. VO (EG) 1257/1999. Kapitel 6: Agrarumweltmaßnahmen - Kapitel VI der VO (EG) Nr. 1257/1999. Bundesforschungsanstalt für Landwirtschaft (FAL)

Reiter K, Roggendorf W, Pufahl A, Essmann S, Horlitz T, Sander A (2003b) Halbzeitbewertung von PROLAND Niedersachen. Kapitel 6: Agrarumweltmaßnahmen - Kapitel VI der VO (EG) Nr. 1257/1999 Bundesforschungsanstalt für Landwirtschaft (FAL)

Reiter K, Roggendorf W, Pufahl A, Essmann S, Horlitz T, Sander A (2003c) Halbzeitbewertung des Hessischen Entwicklungsplans für den ländlichen Raum. Kapitel 6: Agrarumweltmaßnahmen - Kapitel VI der VO (EG) Nr. 1257/1999, Materialband. Bundesforschungsanstalt für Landwirtschaft (FAL)

Reiter K, Roggendorf W, Runge T, Schnaut G (2006a) Aktualisierung der Halbzeitbewertung des NRW-Programms Ländlicher Raum. Kapitel 6: Agrarumweltmaßnahmen - Kapitel VI der VO (EG) Nr. 1257/1999. Bundesforschungsanstalt für Landwirtschaft (FAL):

http://miraculix.fal.de/fallitdok_extern/bitv/dk036929.pdf. Stand 9.1.2008

Reiter K, Roggendorf W, Runge T, Schnaut G (2006b) Aktualisierung der Halbzeitbewertung des Hessischen Entwicklungsplans für den ländlichen Raum. Kapitel 6: Agrarumweltmaßnahmen - Kapitel VI der VO (EG) Nr. 1257/1999. Bundesforschungsanstalt für Landwirtschaft (FAL): http://miraculix.fal.de/fallitdok_extern/bitv/dk037261.pdf. Stand 9.1.2008

Reiter K, Roggendorf W, Runge T, Schnaut G (2006c) Aktualisierung der Halbzeitbewertung von PROLAND Niedersachsenprogramm zur Entwicklung der Landwirtschaft und des Ländlichen Raumes. Kapitel 6: Agrarumweltmaßnahmen - Kapitel VI der VO (EG) Nr. 1257/1999. Bundesforschungsanstalt für Landwirtschaft (FAL): http://miraculix.fal.de/fallitdok_extern/bitv/dk036920.pdf. Stand 9.1.2008

ROBERTS MJ (2008) Do decoupled payments stimulate production? Estimating the effect on program crop acreage using matching (unpublished)

Roodman D (2006) How to do xtabond2: An introduction to "Difference" and "System" GMM in Stata. Centre for Global Development Working Paper 103

Roodman D (2008) A note on the theme of too many instruments. Centre for Global Development Working Paper 125

RoSENBAUM PR (1989) Optimal matching for observational studies. Journal of the Royal Statistical Society 84 (408), 1024-1032 
RosenBAUM PR (1991) A characterization of optimal designs for observational studies. Journal of the Royal Statistical Society 53 (3), 597-610

Rosenbaum PR, RUBIN DB (1983) The central role of propensity score in observational studies for causal effects. Biometrica 70 (1), 41-55

RöSTI A (1997) Auswirkungen der Agrarpolitik 2002 auf die Schweizer Landwirtschaft. Dissertation (ETH Zürich)

RUBIN DB (1974) Estimating causal effects of treatments in randomized and nonrandomized studies. Journal of Educational Psychology 66, 688-701

SÄBL, Statistische ÄMTER Des Bundes UND DER LÄNDER (2004) Statistik regional. Daten für die Kreise und kreisfreien Städte Deutschlands (CD-ROM)

SÄBL, StATistische ÄMTER Des Bundes UND DER LÄNDER (2005) Statistik regional. Daten für die Kreise und kreisfreien Städte Deutschlands (CD-ROM)

SAdoulet E, DE JANVRY A (1995a) Quantitative development analysis. Chapter 3: The profit function approach to supply and factor demand

SAdoulet E, DE JANVRY A (1995b) Quantitative development analysis. Chapter 6: Household Models

SALHOFER K, GLEBE TW (2006) EU agri-environment programs and the "restaurant table effect". Discussion Paper 04-2006, Technical University of Munich, Environmental Economics, Resource Economics and Agricultural Policy

SALHOFER K, STREICHER G (2005) Self-selection as a problem in evaluation agri-environmental programs. In: ORTNER KM (Hrsg.) Assessing rural development policies of the Common Agricultural Policies. Selection of papers from the $87^{\text {th }}$ EAAE-Seminar of the European Association of Agricultural Economics (EAAE), 203-215

SARGAN J (1958) The estimation of economic relationships using instrumental variables. Econometrica 26 (3), 393-415

SAS (2003) SAS Statistical Software, 9.1.3, Service Pack 4. Lizenziert für Bundesministerium für Ernährung, Landwirtschaft und Verbraucherschutz

Schmitt G, Andermann G (1996) Agrarpreise und betrieblicher Strukturwandel in der Landwirtschaft. Agrarwirtschaft 45 (8/9), 323-333

Schmitt G, Schulz-Greve W, LeE M (1995) Familien- und/oder Lohnarbeitskräfte in der Landwirtschaft? Das ist hier die Frage. Ein vernachlässigter Aspekt der Theorie des landwirtschaftlichen Faktoreinsatzes. Diskussionsbeiträge 9550

SCHULZ-GREVE W (1994) Die Zeitallokation landwirtschaftlicher Haushalte: Eine theoretische und empirische Analyse. Interdisziplinäre Studien zur Entwicklung in ländlichen Räumen 7 
SEMYKINA A, WoOLdRIDGE JM (2005): Estimating panel data models in the presence of endogeneity and selection: Theory and application. Working Paper. Michigan State University.

http://www.msu.edu/ ec/faculty/wooldridge/current\%20research/selectivje.pdf Stand 12.2.2008

Serra T, Goodwin BK, Featherstone AM (2005) Agricultural policy reform and offfarm labour decisions. Journal of Agricultural Economics 56 (2), 271-285

SHAIK S, HELMERS G (2006) An examination of farm program payments on farm economic structure. Research in Agricultural and Applied Economics: http://agecon.lib.umn.edu/. Stand 26.7.2007

Shapiro D, Bollmann RD, Ehrensaft P (1987) Farm size growth in Canada. American Journal of Agricultural Economics 69, 477-483

SinABell F, STREICHER G (2005) Programme evaluation with micro-data: the use of FADN data to evaluate effects on the market situation of programme participants. In: ORTNER KM (Hrsg.) Assessing rural development policies of the Common Agricultural Policies. Selection of papers from the $87^{\text {th }}$ EAAESeminar of the European Association of Agricultural Economics (EAAE), 267280

Singh I, Squire L, Strauss J (1986) The Basic Model: Theory, empirical results and policy conclusions. In: Singh I, SQuire L, Strauss J (Hrsg.) Agricultural household models. Extensions, applications, and policy. Published for the World Bank

Singer JD, WiLlett JB (2003) Applied longitudinal data analysis. New York

SMith JA, TODD PE (2005) Does matching overcome LaLonde's critique of nonexperimental estimators? Journal of Econometrics 125, 305-353

StataCorp. (2007) Stata Statistical Software: Release 10. College Station, TX: StataCorp LP

STATISTISCHE ÄMTER (2007) Mikrodaten der Agrarstatistik: Landwirtschaftszählung (1999) und Agrarstrukturerhebung (2003). Forschungsdatenzentrum: www.forschungsdatenzentrum.de. Stand 6.1.2007

Statistisches Bundesamt (div. Jgg.) Preisindizes für die Land- und Forstwirtschaft. Fachserie 17, Reihe 1

Statistisches Bundesamt (div. Jgg.) Statistisches Jahrbuch über Ernährung, Landwirtschaft und Forsten

Sumner DA, LEIBY JD (1987) An econometric analysis of the effects of human capital on size and growth among dairy farms. American Journal of Agricultural Economics 69, 465-470 
UptON M, Haworth S (1987) The growth of farms. European Review of Agricultural Economics 14, 351-366

V. AUER L (2003) Ökonometrie. Eine Einführung

VAnselmbrouck I. V. Hylenbroeck G, Verbeke W (2002) Determinants of the willingness of Belgian farmers to participate in agri-environmental measures. Journal of Agricultural Economics 53 (3), 489-511

VERBEEK M, NiJMAN T (1992) Testing for selectivity bias in panel models. International Economic Review 33 (3), 681-703

WeISS CR (1999) Farm Growth and Survival. Econometric evidence of individuals farms in upper Austria. American Journal of Agricultural Economics 81, 103-116

WeISS F (2006a) Bestimmungsgründe für die Aufgabe/Weiterführung landwirtschaftlicher Betriebe in Österreich. Diskussionspapier des Instituts für nachhaltige Wirtschaftsentwicklung DP-14-2006

WeISS F (2006b) Ursachen für den Erwerbsartenwechsel in landwirtschaftlichen Betrieben Österreichs. Diskussionspapier des Instituts für nachhaltige Wirtschaftsentwicklung DP-18-2006

WestscotT PC, Price JM (2001) Analysis on the U.S. commodity loan program with marketing loan provisions. Agricultural Economic Report 801

WILSON GA (1997) Factors influencing farmer participation in the environmentally sensitive area scheme. Journal of Environmental Management 50, 67-93

WINDMEIJER F (2000) A finite sample correction for the variance of linear two-step GMM estimators. The Institute for Fiscal Studies Working Paper W00/19

WiTZKE HP (1993) Mikrotheorie in der Agrarsektoranalyse - Neoklassischer Standard, Konflikte mit der Realität und Versöhnungsversuche mit Komplikationen. Volkswirtschaftliche Schriften 431

WoOLDRIDGE JM (1995) Selection corrections for panel data models under conditional mean independence assumption. Journal of Econometrics 68, 115-132

WoOldRIDGE JM (2002) Econometric Analysis of Cross Section and Panel Data. Cambridge (MIT Press)

WOSSINK GAA, V. WENUM JH (2003) Biodiversity conservation by farmers: analysis of actual and contingent participation. European Review of Agricultural Economics 30 (4), 461-485

WTO World Trade Organization (1994) Agreement on Agriculture

Wynn G, Crabtree B, Potts J (2001) Modelling farmers entry into the environmentally sensitive area schemes in Scotland. Journal of Agricultural Economics 52 (1), $65-82$ 
XiANPING L, LYNCH L (2006) Agricultural preservation programs affect farmland conversion? Evidence from a propensity score matching estimator. Department of Agricultural and Resource Economics Working Paper 06-08. University of Maryland, College ParkSome

Young CE, Westscott, PC (2000) How decoupled is US agricultural support for major crops. American Journal of Agricultural Economics 82 (3), 762-767

ZALF, ZENTRUM FÜR AGRARLANDSCHAFTS- UND LANDNUTZUNGSFORSCHUNG E.V. (2005a): Aktualisierung der Halbzeitbewertung des Plans zur Entwicklung des ländlichen Raumes gemäß VO (EG) Nr. 1257/1999 des Landes Berlin. Im Auftrag der Senatsverwaltung für Wirtschaft, Arbeit und Frauen. ZALF: www.zalf.de/home_zalf/download/soz/KULAP2_endbericht_berlin.pdf. Stand 22.6.2006

ZALF, ZENTRUM FÜR AGRARLANDSCHAFTS- UND LANDNUTZUNGSFORSCHUNG E.V. (2005b) Aktualisierung der Halbzeitbewertung des Plans zur Entwicklung des ländlichen Raumes gemäß VO (EG) Nr. 1257/1999 des Landes Brandenburg. Im Auftrag des Ministeriums für Ländliche Entwicklung, Umwelt und Verbraucherschutz des Landes Brandenburg

Gesetz über die Gemeinschaftsaufgabe "Verbesserung der Agrarstruktur und des Küstenschutzes" (GAK-Gesetz - GAKG) in der Fassung der Bekanntmachung vom 21. Juli 1988 (BGBl. I S. 1055), zuletzt geändert durch das Gesetz zur Modulation von Direktzahlungen im Rahmen der Gemeinsamen Agrarpolitik und zur Änderung des GAK-Gesetzes vom 10. Mai 2002 (BGBl. I S.1527)

Richtlinie des Rates über die Landwirtschaft in Berggebieten und bestimmten Benachteiligten Gebieten (Mai 1995)

Erklärung von Cork. Ein dynamischer ländlicher Raum. Europäische Konferenz über Ländliche Entwicklung vom 7. bis 9. November 1996 in Cork, Irland

Verordnung (EG) Nr. 1257/1999 des Rates vom 17. Mai 1999 über die Förderung der Entwicklung des ländlichen Raums durch den Europäischen Ausrichtungs- und Garantiefonds für die Landwirtschaft (EAGFL) und zur Änderung bzw. Aufhebung bestimmter Verordnungen 


\section{Anhang}

\section{Tabellen}




\section{Verzeichnis der Tabellen im Anhang}

Tabelle A1: $\quad$ Empirische Untersuchungen zu den Wirkungen von direkten Transferzahlungen an landwirtschaftliche Betriebe

Tabelle A2: $\quad$ Definition der verwendeten Buchführungsdaten (2000 bis 2005)

Tabelle A3:

Definition der verwendeten Kontextdaten

Tabelle A4:

Deskriptive Statistik, Matching-Analyse

Tabelle A5: $\quad$ Standardisierter Bias vor und nach dem Matching für die in der Logit-Analyse verwendeten erklärenden Variablen, Deutschland gesamt

Tabelle A6: $\quad$ Effekte der AUM- und AZ-Teilnahme in Abhängigkeit von der Höhe der Programmzahlungen je Betrieb

Tabelle A7: $\quad$ Effekt der Ausgleichszulage auf das Flächenwachstum in Abhängigkeit vom Anteil der betrieblichen LF im benach teiligten Gebiet

Tabelle A8: $\quad$ Probit-Modelle zur Schätzung der Wahrscheinlichkeit der AUM-Teilnahme

Tabelle A9: $\quad$ Probit-Modelle zur Schätzung der Wahrscheinlichkeit der AZ-Teilnahme

Tabelle A10: $\quad$ Zusammenhang zwischen dem Alter des Betriebsleiters und der Teilnahme an Agrarumweltmaßnahmen, Chi-Quadrat Test

Tabelle A11: $\quad$ Effekt der AUM-Teilnahme auf das Flächenwachstum (vor und nach 2003) in Abhängigkeit von der Betriebsform 
Tabelle A1: Empirische Untersuchungen zu den Wirkungen von direkten Transferzahlungen an landwirtschaftliche Betriebe

\begin{tabular}{|c|c|c|c|c|c|c|c|c|c|c|c|}
\hline Autor(en) & $\begin{array}{l}\text { Art der direk- } \\
\text { ten Transfer- } \\
\text { zahlung }\end{array}$ & Betrachtungsrahmen & Theoretischer Ansatz & Methode & 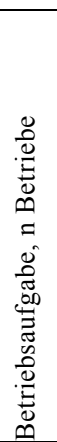 & 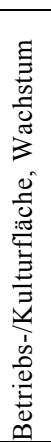 & 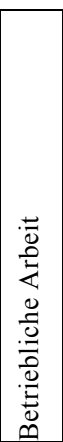 & 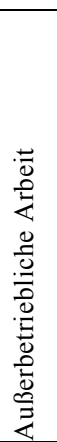 & 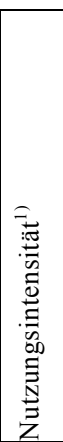 & 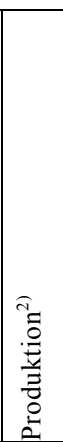 & 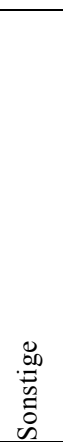 \\
\hline ADAMS et al. (2001) & Entkoppelte DZ & Nuts I, US, 1997-2000 & keine Angabe & $\begin{array}{l}\text { Kontrollvariablen, } \\
\text { Differenzierung, SU }\end{array}$ & & & & & & + & \\
\hline AHEARN et al. (2006) & $\begin{array}{l}\text { Entkoppelte DZ } \\
\text { Gekoppelte DZ } \\
\text { Umwelt (CRP) } \\
\end{array}$ & $\begin{array}{l}\text { Einzelbetrieb, US, 1996, } \\
1999\end{array}$ & Separables UHM & $\begin{array}{l}\text { Kontrollvariablen, } \\
\text { SO, Betriebslei- } \\
\text { ter/Partnerin }\end{array}$ & & & & $\begin{array}{l}-/- \\
-/ 0 \\
0 /-\end{array}$ & & & \\
\hline AHEARN et al. (2002) & $\begin{array}{l}\text { Gekoppelte DZ } \\
\text { Umwelt (CRP) }\end{array}$ & $\begin{array}{l}\text { Nuts I, US, 1982, 1987, } \\
1992,1996\end{array}$ & $\begin{array}{l}\text { Theoretische Ansätze zum agrarstruk- } \\
\text { turellen Wandel, Hypothesentest }\end{array}$ & Kontrollvariablen, SO & + & + & & $\overline{0}$ & & & $++^{4)}$ \\
\hline AHEARN et al. (2005) & $\begin{array}{l}\text { Gekoppelte DZ } \\
\text { Umwelt (CRP) }\end{array}$ & $\begin{array}{l}\text { Nuts I, US, 1982, 1987, } \\
\text { 1992, } 1996\end{array}$ & Separables UHM & $\begin{array}{l}\text { Kontrollvariablen, } \\
\text { SO, kleine/große } \\
\text { Betriebe }\end{array}$ & $\begin{array}{l}0 / \\
+ \\
+/-\end{array}$ & & & $\begin{array}{l}0 \\
0\end{array}$ & & & $\begin{array}{l}+^{4)} \\
+^{4)}\end{array}$ \\
\hline BECK \& DOGOT (2006) & Struktur (AFP) & $\begin{array}{l}\text { Einzelbetrieb (Milchvieh), } \\
\text { Wallonien, BEL, 1999- } \\
2004\end{array}$ & keine Angabe & $\begin{array}{l}\text { Kreuzvergleich, } \\
\text { Schichtung, SO * }\end{array}$ & & + & + & & & & $\begin{array}{c}+^{3)} \\
5)\end{array}$ \\
\hline BRÜMMER \& LOY (2000) & Struktur (AFP) & $\begin{array}{l}\text { Einzelbetrieb (Milchvieh), } \\
\text { Schleswig-Holstein, D, } \\
\text { 1987-1994 }\end{array}$ & Produktionstheorie & Kontrollvariablen, SU & & & & & & & $-10)$ \\
\hline BURGATh et al (2001) & Struktur (AFP) & \begin{tabular}{|l} 
Einzelbetrieb, D, 1994- \\
1999
\end{tabular} & keine Angabe & $\begin{array}{l}\text { Kreuzvergleich, } \\
\text { Schichtung, SO }\end{array}$ & & & & & & & $+*^{* 3}$ \\
\hline $\begin{array}{l}\text { CHANG \& BOISVERT } \\
(2005)\end{array}$ & $\mathrm{DZ}$ & Einzelbetrieb, US, 2001 & $\begin{array}{l}\text { Non-separable UHM (mit expliziter } \\
\text { Berücksichtigung flächengebundener } \\
\text { Zahlungen) }\end{array}$ & Kontrollvariablen, SO & & & & - & & & \\
\hline CHANG et al. (2008) & Umwelt (CRP) & Einzelbetrieb, US, 2003 & Separables UHM & Kontrollfunktion, SO & & & & & & & $-3)$ \\
\hline EL-OstA et al. (2004) & $\begin{array}{l}\text { Entkoppelte DZ } \\
\text { Gekoppelte DZ } \\
\text { Sonstige } \\
\end{array}$ & Einzelbetrieb, US, 2001 & Separables UHM & $\begin{array}{l}\text { Instrument Variablen, } \\
\text { Kontrollvariablen, SO }\end{array}$ & & & $\begin{array}{l}+ \\
0 \\
+ \\
\end{array}$ & $\begin{array}{l}- \\
- \\
0\end{array}$ & & & \\
\hline
\end{tabular}




\begin{tabular}{|c|c|c|c|c|c|c|c|c|c|c|c|}
\hline Autor(en) & $\begin{array}{l}\text { Art der direk- } \\
\text { ten Transfer- } \\
\text { zahlung }\end{array}$ & Betrachtungsrahmen & Theoretischer Ansatz & Methode & 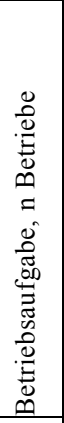 & 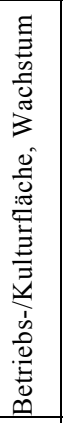 & 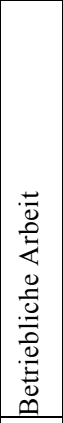 & 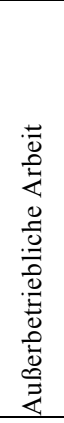 & 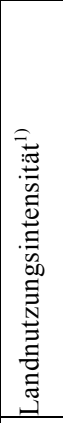 & 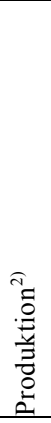 & 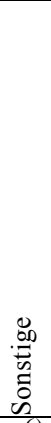 \\
\hline ESPOSTI (2007) & $\mathrm{DZ}$ & Nuts I, EU, 1989-2000 & Separables UHM & $\begin{array}{l}\text { IV-Modell (GMM), } \\
\text { Differenzierung, SU }\end{array}$ & & & & & & & $-6)$ \\
\hline GLAUBEN et al. (2005) & $\mathrm{DZ}$ & $\begin{array}{l}\text { Nuts I (ohne ehem. DDR), } \\
\text { D, 1991, } 1999\end{array}$ & Separables UHM (implizit) & Kontrollvariablen, SO & 0 & & & & & & \\
\hline $\begin{array}{l}\text { GOETZ \& DEBERTIN } \\
(2001)\end{array}$ & $\mathrm{DZ}$ & Nuts III, US, 1987-1997 & Separables UHM & Kontrollvariablen, SO & + & & & & & & \\
\hline GIRANTE et al. (2008) & Entkoppelte DZ & $\begin{array}{l}\text { Einzelbetrieb, US, 1996- } \\
2001\end{array}$ & Produktionstheorie & $\begin{array}{l}\text { Kontrollvariablen, } \\
\text { Differenzierung, SU }\end{array}$ & & & & & & + & \\
\hline $\begin{array}{l}\text { GOODWIN \& MISHRA } \\
(2004)\end{array}$ & Entkoppelte DZ & Einzelbetrieb, US, 2001 & Separables UHM & Kontrollvariablen, SO & & & & - & & & \\
\hline GooDwIN et al. (2007) & $\begin{array}{l}\text { Entkoppelte DZ } \\
\text { Gekoppelte DZ } \\
\text { Sonstige DZ } \\
\text { Umwelt (CRP) } \\
\end{array}$ & $\begin{array}{l}\text { Einzelbetrieb, US, 2003, } \\
2004\end{array}$ & Separables UHM & $\begin{array}{l}\text { IV-Modell, Kontroll- } \\
\text { variablen, SO, Be- } \\
\text { triebsleiter/Partnerin }\end{array}$ & & $\begin{array}{l}- \\
+ \\
+\end{array}$ & & $\begin{array}{l}0 /- \\
0 /+ \\
0 / 0 \\
0 / 0\end{array}$ & & & $\begin{array}{l}0^{7)} \\
0^{7)} \\
0^{7)}\end{array}$ \\
\hline HOFER (2002) & Entkoppelte DZ & $\begin{array}{l}\text { Einzelbetrieb, Kanton Bern } \\
(\mathrm{CH}), 1994-1998\end{array}$ & Separables UHM & Kontrollvariablen, SO & - & & & - & & & \\
\hline $\begin{array}{l}\text { HUFFMAN \& EVENSON } \\
(2001)\end{array}$ & Gekoppelte DZ & Nuts I, US, 1950-1982 & $\begin{array}{l}\text { Hypothesentests zu den Gründen des } \\
\text { agrarstrukturellen Wandels }\end{array}$ & \begin{tabular}{|l|}
$\begin{array}{l}\text { nicht relevant (Sektor- } \\
\text { studie) }\end{array}$ \\
\end{tabular} & & + & & - & & & \\
\hline KEY \& ROBERTS (2003) & $\mathrm{DZ}$ & $\begin{array}{l}\text { Einzelbetrieb (Markt- } \\
\text { frucht), US, 1987, 1992, } \\
1997\end{array}$ & $\begin{array}{l}\text { Neoklassisches Modell für Größe ldw. } \\
\text { Betriebe (KISLEV und PETERSON in } \\
\text { HARRINGTON und REINSEL, 1995) }\end{array}$ & Kontrollvariablen, SO & - & $\begin{array}{c}+/ \\
0\end{array}$ & & & & & \\
\hline KEY \& ROBERTS (2006) & $\mathrm{DZ}$ & $\begin{array}{l}\text { Einzelbetrieb (Markt- } \\
\text { frucht), US, 1978-1997 }\end{array}$ & $\begin{array}{l}\text { Evolutionäre Ansätze (JOVANOVIC, } \\
\text { 1982) }\end{array}$ & Kontrollvariablen, SO & - & & & & & & \\
\hline KEY et al. (2005) & $\mathrm{DZ}$ & $\begin{array}{l}\text { Einzelbetrieb (Markt- } \\
\text { frucht), US, 1987, 1992, } \\
1997,2002\end{array}$ & $\begin{array}{l}\text { Neoklassische Theorie, Hypothesen- } \\
\text { tests }\end{array}$ & Kontrollvariablen, SU & & + & & & & + & \\
\hline KIM et al. (2005) & DZ & Sektor, US, 1960-1996 & $\begin{array}{l}\text { Innovationstheorie, Neoklassische } \\
\text { Produktionstheorie }\end{array}$ & $\begin{array}{l}\text { nicht relevant (Sektor- } \\
\text { studie) }\end{array}$ & $+/-$ & & & & & & \\
\hline
\end{tabular}




\begin{tabular}{|c|c|c|c|c|c|c|c|c|c|c|c|}
\hline Autor(en) & $\begin{array}{l}\text { Art der direk- } \\
\text { ten Transfer- } \\
\text { zahlung }\end{array}$ & Betrachtungsrahmen & Theoretischer Ansatz & Methode & 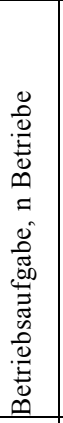 & 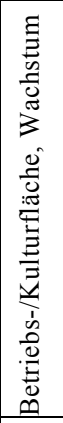 & 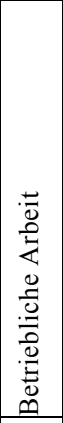 & 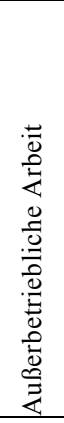 & 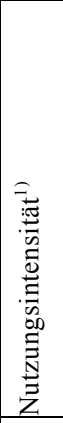 & 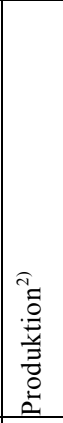 & 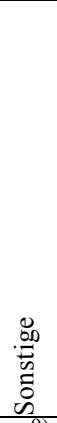 \\
\hline $\begin{array}{l}\text { LAMBERT \& GRIFFIN } \\
(2004)\end{array}$ & $\mathrm{DZ}$ & $\begin{array}{l}\text { Einzelbetrieb, Illinois (US), } \\
\text { 1996-2001 }\end{array}$ & Neoklassische Produktionstheorie & $\begin{array}{l}\text { FE-Modell (mit zeitli- } \\
\text { chen und räumlichen } \\
\text { Lags), SU }\end{array}$ & & + & + & & - & & $++^{9)}$ \\
\hline LAMBERT et al. (2006) & \begin{tabular}{|l|} 
Entkoppelte DZ, \\
Umwelt (CRP)
\end{tabular} & $\begin{array}{l}\text { Einzelbetrieb (Ackerbau), } \\
\text { US, } 2004\end{array}$ & $\begin{array}{l}\text { Verhaltensorientierter Ansatz, } \\
\text { Hypothesentest }\end{array}$ & IV-Modell, SO & & & & & & & $\begin{array}{r}0^{8)} \\
+{ }^{8)}\end{array}$ \\
\hline LENCE \& MiSHRA (2003) & $\mathrm{DZ}$ & $\begin{array}{l}\text { Nuts III, Iowa (US), } \\
1997,1998\end{array}$ & Neoklassische Produktionstheorie & \begin{tabular}{|l|}
$\begin{array}{l}\text { Kontrollvariablen, SU } \\
\text { (Spatial Lags) }\end{array}$ \\
\end{tabular} & & & & & & & $++^{9)}$ \\
\hline LYNCH et al. (2007b) & Umwelt (FPP) & $\begin{array}{l}\text { Nuts III, Maryland (US), } \\
1997-2003\end{array}$ & keine Angabe & $\begin{array}{l}\text { Kreuzvergleich, } \\
\text { Matching, SO }\end{array}$ & & & & & & & $\begin{array}{l}0^{9)} \\
+9) \\
\end{array}$ \\
\hline MANN (2003) & Entkoppelte DZ & Nuts I, CH, 1995-2001 & $\begin{array}{l}\text { Hypothesentest zum agrarstrukturellen } \\
\text { Wandel }\end{array}$ & $\begin{array}{l}\text { Kontrollvariablen, } \\
\text { SO }\end{array}$ & + & & 0 & & & & \\
\hline $\begin{array}{l}\text { MISHRA \& GOODWIN } \\
(1997)\end{array}$ & Entkoppelte DZ & $\begin{array}{l}\text { Einzelbetrieb, Kansas (US), } \\
1991,1992\end{array}$ & Separables UHM & Kontrollvariablen, SO & & & & $-/-$ & & & \\
\hline MORO \& SCKOKAI (1999) & Gekoppelte DZ & $\begin{array}{l}\text { Einzelbetrieb, ITA, } \\
\text { 1993-1995 }\end{array}$ & Neoklassische Produktionstheorie & Kontrollvariablen & & + & & & $\begin{array}{c}+/- \\
10 \\
\end{array}$ & + & \\
\hline $\begin{array}{l}\text { NIEBERG (1996), } \\
\text { NIEBERG \& OFFERMANN } \\
(2006)\end{array}$ & Umwelt (Öko) & $\begin{array}{l}\text { Einzelbetrieb, D, verschie- } \\
\text { dene Zeiträume }\end{array}$ & keine Angabe & \begin{tabular}{|l|} 
Kreuzvergleich, \\
Kovariate Matching, \\
SO *
\end{tabular} & & & + & & - & - & $++^{3)}$ \\
\hline $\begin{array}{l}\text { OFFERMANN \& NIEBERG } \\
(2001 ; 2003)\end{array}$ & Umwelt (Öko) & $\begin{array}{l}\text { Einzelbetrieb, EU, D, 1993- } \\
\text { 2003* }\end{array}$ & keine Angabe & \begin{tabular}{|l|} 
Kreuzvergleich, Ko- \\
variate Matching, SO* \\
\end{tabular} & & & + & & & & $++^{3)}$ \\
\hline OLTMER (2001) & $\mathrm{DZ}$ & $\begin{array}{l}\text { Einzelbetrieb \& aggregiert, } \\
17 \text { Studien (1942-1993), } \\
\text { vorwiegend US \& CAN }\end{array}$ & $\begin{array}{l}\text { Finanztheorie, } \\
\text { Hedonisches Preismodell }\end{array}$ & $\begin{array}{l}\text { nicht relevant (Meta- } \\
\text { analyse) }\end{array}$ & & & & & & & 9) \\
\hline $\begin{array}{l}\text { OSTERBURG \& } \\
\text { STRATMANN (2002), } \\
\text { OSTERBURG (2005) } \\
\end{array}$ & $\begin{array}{l}\text { Umwelt (AUM) } \\
\text { Umwelt (Öko) }\end{array}$ & $\begin{array}{l}\text { Einzelbetrieb, Bundeslän- } \\
\text { der (D), 1990/91-1999/2000 } \\
\text { bzw. 2001/02 }\end{array}$ & keine Angabe & $\begin{array}{l}\text { DiD-Vergleich, Euk- } \\
\text { lidische Distanz } \\
\text { Matching, SU } \\
\end{array}$ & & $\begin{array}{l}0 \\
+\end{array}$ & & & $\begin{array}{l}- \\
-\end{array}$ & & $\begin{array}{c}+/ 0 \\
+ \\
3) \\
\end{array}$ \\
\hline PFEFFERLi (2006) & Struktur (AFP) & $\begin{array}{l}\text { Einzelbetrieb, CH, 1999, } \\
2002\end{array}$ & keine Angabe & $\begin{array}{l}\text { Vorher-Nachher- } \\
\text { Vergleich, Schich- } \\
\text { tung, SO * }\end{array}$ & & + & - & & & & $++^{3)}$ \\
\hline
\end{tabular}




\begin{tabular}{|c|c|c|c|c|c|c|c|c|c|c|c|}
\hline Autor(en) & $\begin{array}{l}\text { Art der direk- } \\
\text { ten Transfer- } \\
\text { zahlung }\end{array}$ & Betrachtungsrahmen & Theoretischer Ansatz & Methode & 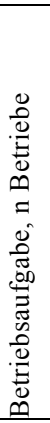 & 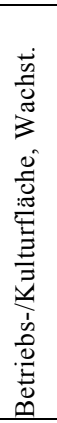 & 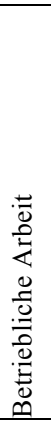 & 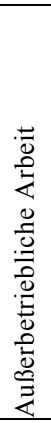 & 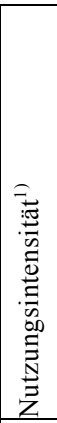 & 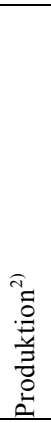 & 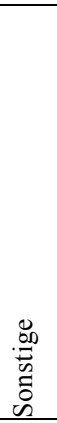 \\
\hline Pietola et al. (2003) & Gekoppelte DZ & $\begin{array}{l}\text { Einzelbetrieb, FIN, 1993- } \\
1998\end{array}$ & Separables UHM & Kontrollvariablen, SO & + & & & & & & \\
\hline PLANKL et al. (2005) & Struktur (AZ) & $\begin{array}{l}\text { Einzelbetrieb, Bundeslän- } \\
\text { der, 2001, } 2004\end{array}$ & keine Angabe & $\begin{array}{l}\text { Kreuzvergleich, } \\
\text { Schichtung, SO * }\end{array}$ & & & & & & & $++^{3)}$ \\
\hline PUfAHL \& WEISS (2009) & $\begin{array}{l}\text { Umwelt (AUM) } \\
\text { Struktur (AZ) }\end{array}$ & $\begin{array}{l}\text { Einzelbetrieb, D, 2000- } \\
2005\end{array}$ & Separables UHM (implizit) & $\begin{array}{l}\text { DiD-Vergleich, Propen- } \\
\text { sity Score Matching, SU }\end{array}$ & & $\begin{array}{l}+ \\
+\end{array}$ & $\begin{array}{l}+ \\
0\end{array}$ & $\begin{array}{l}0 \\
0\end{array}$ & $\overline{0}$ & $\begin{array}{l}+ \\
+\end{array}$ & \\
\hline RÖSTI (1997) & Entkoppelte DZ & $\begin{array}{l}\text { Nuts III, Kanton Bern }(\mathrm{CH}) \text {, } \\
\text { 1990-1995 }\end{array}$ & keine Angabe & Kontrollvariablen, SO & + & & & & & & \\
\hline ROBERTS (2008) & Entkoppelte DZ & $\begin{array}{l}\text { Einzelbetrieb, US, 1997- } \\
2002\end{array}$ & Neoklassische (Nutzen-) Theorie & $\begin{array}{l}\text { DiD-Vergleich, Propen- } \\
\text { sity Score Matching, SU }\end{array}$ & & & & & & + & \\
\hline $\begin{array}{l}\text { SALHOFER \& STREICHER } \\
(2005)\end{array}$ & $\begin{array}{l}\text { Umwelt (AUM) } \\
\text { Umwelt (Öko) }\end{array}$ & $\begin{array}{l}\text { Einzelbetrieb, AUT, 1994- } \\
1997\end{array}$ & keine Angabe & FE-Modell, SU & & & & & & $\begin{array}{l}0 \\
-\end{array}$ & \\
\hline SERRA et al. (2005) & $\begin{array}{l}\text { Entkoppelte DZ } \\
\text { Gekoppelte DZ }\end{array}$ & $\begin{array}{l}\text { Einzelbetrieb, Kansas (US), } \\
\text { 1994-2000 }\end{array}$ & Separables UHM & Kontrollvariablen, SO & & & & $\overline{0}$ & & & \\
\hline $\begin{array}{l}\text { SHAIK \& HELMERS } \\
(2006)\end{array}$ & $\mathrm{DZ}$ & $\begin{array}{l}\text { Sektor, Nebraska (US), } \\
\text { 1936-2004 }\end{array}$ & Neoklassische Produktionstheorie & $\begin{array}{l}\text { nicht relevant (Sektor- } \\
\text { studie) }\end{array}$ & & + & 0 & & & & \\
\hline $\begin{array}{l}\text { SINABELL \& STREICHER } \\
(2005)\end{array}$ & $\begin{array}{l}\text { Struktur (AFP) } \\
\text { Umwelt (Öko) }\end{array}$ & $\begin{array}{l}\text { Einzelbetrieb, AUT, 1998- } \\
2002\end{array}$ & keine Angabe & $\begin{array}{l}\text { DiD-Vergleich (ohne } \\
\text { Matching), SU }\end{array}$ & $\begin{array}{l}+ \\
0\end{array}$ & & & & & & $\begin{array}{l}+3) \\
0^{3)} \\
\end{array}$ \\
\hline WEISS (2006a; 2006b) & $\begin{array}{l}\text { Umwelt (AUM) } \\
\text { Struktur (AZ) } \\
\text { DZ, AUM, AZ }\end{array}$ & $\begin{array}{l}\text { Einzelbetrieb, AUT, 1995, } \\
1999\end{array}$ & Separables UHM (implizit) & Kontrollvariablen, SO & $\begin{array}{l}- \\
0 \\
-\end{array}$ & & + & & & & \\
\hline
\end{tabular}

${ }^{1)}$ Viehbesatzdichte, Pflanzenschutz- und Düngemittelaufwand, ${ }^{2)}$ Anbaufläche geförderter Kulturen, tierische Leistung, pflanzlicher Ertrag, Umsatz, ${ }^{3)}$ Landwirtschaftliches Einkommen, ${ }^{4)}$ Faktorproduktivität, ${ }^{5)}$ Milchquote, ${ }^{6}$ Veränderung der Bruttowertschöpfung je Erwerbstätigen, ${ }^{7}$ Diversifizierung landwirtschaftlicher Aktivitäten ${ }^{8)}$ Anteil umweltfreundlich bewirtschafteter LF, ${ }^{9}$ Pacht- und Bodenpreis, ${ }^{10)}$ Effizienz, $0=$ keine Wirkung, $+=$ positive Wirkung, $-=$ negative Wirkung, / = Ergebnisse für Betriebs-/Personengruppen,

leer = keine Untersuchung, * Ergebnisse sind nicht statistisch abgesichert. DZ = Direktzahlungen allgemein, Entkoppelte DZ = Entkoppelte Direktzahlungen (z. B. Agricultural

Marketing Transition Assistance (AMTA), Production Flexibility Payments (PFC)), Gekoppelte DZ = Gekoppelte Direktzahlungen (z. B. Marketing Loan Program (MLP), Loan

Deficiency Payments (LDP), Marketing Loss Assistance Payments (MLA) und andere), Struktur = Agrarstrukturpolitische Programme, Umwelt = Agrarumweltpolitische Programme, $\mathrm{E}=$ Einzelbetriebliche Analyse, A = Aggregierte Analyse, Nuts III = Kreis, US County, Nuts I = Bundesland, US State), CRP = Conservation Reserve Program, AFP = Agrarinvestitionsförderung, AUM = Agrarumweltmaßnahmen (ohne Ökologischen Landbau), Öko = AUM Ökologischer Landbau, AZ = Ausgleichszulage für benachteiligte Gebiete, FPP $=$ Farmland Preservation Program, $\mathrm{SO}=$ Selection on observables, $\mathrm{SU}=$ Selection on unobservables

Quelle: eigene Zusammenstellung nach angegebenen Quellen. 
Tabelle A2: $\quad$ Definition der verwendeten Buchführungsdaten (2000 bis 2005)

\begin{tabular}{|c|c|}
\hline Variable & Beschreibung \\
\hline \multicolumn{2}{|l|}{ Betriebliche Merkmale } \\
\hline Ackerbau & Ackerbaubetrieb \\
\hline AK Betrieb & Arbeitseinsatz im ldw. Betrieb (Familien- und Fremd-AK) \\
\hline Alter Betriebsleiter & Alter des Betriebsleiters \\
\hline Anteil Grünland & Anteil Grünland an LF \\
\hline Anteil LF in benacht. Gebiet & Anteil LF des Betriebes im benachteiligten Gebiet \\
\hline Anteil Pachtland & Anteil Pachtland an LF Betrieb \\
\hline AUM-Teilnahme & Teilnahme des Betriebes an Agrarumweltmaßnahmen \\
\hline AUM-Zahlungen & Zahlungen im Rahmen von Agrarumweltmaßnahmen je Betrieb \\
\hline AUM-Zahlungen (je ha) & Zahlungen im Rahmen von Agrarumweltmaßnahmen je Betrieb (je ha) \\
\hline Außerbetriebliche $A K$ & Außerbetriebliche Arbeit des Betriebsleiterpaares \\
\hline Außerbetriebliche $A K B L$ & Außerbetriebliche Arbeit des Betriebsleiters \\
\hline AZ-Teilnahme & Teilnahme des Betriebes an der Ausgleichszulage \\
\hline AZ-Zahlungen & Höhe der Ausgleichszulage je Betrieb \\
\hline AZ-Zahlungen (je ha) & Höhe der Ausgleichszulage je Betrieb (je ha) \\
\hline Direktzahlungen & Höhe der Tier- und Flächenprämien \\
\hline Ausgaben Dünger (je ha) & Betriebliche Ausgaben für Düngemittel (je ha) \\
\hline Flächenprämie (je ha) & Höhe der Flächenprämien (je ha) \\
\hline Futterbau & Futterbaubetrieb \\
\hline Gemischt & Gemischtbetrieb \\
\hline Gewinn & Um Direktzahlungen, AUM- und AZ-Zahlungen bereinigter Gewinn \\
\hline Grünland & Grünlandbetrieb \\
\hline$H F F$ & Hauptfutterfläche \\
\hline Höhe & Höhe des Betriebes über Normal Null (N. N.) \\
\hline Kapital & Eigen- und Fremdkapital ohne Boden \\
\hline Kapital (je ha) & Eigen- und Fremdkapital ohne Boden (je ha) \\
\hline Ldw. Bildung (Jahre) & Ldw. Berufsausbildung des Betriebsleiters \\
\hline Ldw. Bildung (Techniker) & Ldw. Technikerabschluss des Betriebsleiters \\
\hline Ldw. Bildung (Uni/FH) & Ldw. Uni/FH-Abschluss des Betriebsleiters \\
\hline$L F$ & Landwirtschaftlich genutzte Fläche des Betriebes \\
\hline$L V Z$ & Landwirtschaftliche Vergleichszahl des Betriebes \\
\hline Nebenerwerb & Landwirtschaftlicher Betrieb im Nebenerwerb \\
\hline Nldw. Bildung (Uni/FH) & Nicht-ldw. Uni/FH-Abschluss des Betriebsleiters \\
\hline Ord. Ergebnis & Ordentliches Ergebnis \\
\hline Ord. Ergebnis (je ha) & Ordentliches Ergebnis (je ha) \\
\hline Ord. Ergebnis $+P A / A K$ & Ordentliches Ergebnis + Personalaufwand (je AKE) \\
\hline Ausgaben PSM (je ha) & Betriebliche Ausgaben für Pflanzenschutzmittel (je ha) \\
\hline Rinderbesatz & Rinderbesatz \\
\hline Rinderbesatzdichte & Rinderbesatz je Hauptfutterfläche \\
\hline$R G V / H F F(\operatorname{minmax})$ & $\mathrm{RGV}$ je $\mathrm{HFF}>=0,3<1,4$ \\
\hline Tierprämie (je ha) & Höhe der Tierprämien (je ha) \\
\hline Umsatz & Umsatz \\
\hline Umsatz (je ha) & Umsatz (je ha) \\
\hline Veredlung & Veredlungsbetrieb \\
\hline Viehbestand (je $100 \mathrm{ha}$ ) & Viehbestand (je 100 ha) \\
\hline
\end{tabular}

$\mathrm{AKE}=$ Arbeitskrafteinheiten, $\mathrm{GV}=$ Großvieheinheiten, ha $=$ Hektar.

Quelle: LAND-Data GmbH (2006). 
Tabelle A3: $\quad$ Definition der verwendeten Kontextdaten

\begin{tabular}{llr}
\hline Variable & Definition & Zeitlicher Bezug \\
\hline Regionale Merkmale & & \\
Anteil Futterbau & Anteil der Futterbaubetriebe an allen ldw. Betrieben im Kreis & 1999 1) \\
Anteil Gemischt & Anteil der Gemischtbetriebe an allen ldw. Betrieben im Kreis & 1999 1) \\
Anteil Ackerbau & Anteil der Ackerbaubetriebe an allen ldw. Betrieben im Kreis & 1999 1) \\
Anteil Veredlung & Anteil der Veredlungsbetriebe an allen ldw. Betrieben im Kreis & 1999 1) \\
BIP (pro Kopf) & Bruttoinlandsprodukt (pro Kopf) & 2000 bis 2005 1) \\
BWS Landwirtschaft & Bruttowertschöpfung in der Landwirtschaft & 2000 bis 2005 1) \\
BWS Ldw. (je ha) & Bruttowertschöpfung Landwirtschaft (je ha) & 2000 bis 2005 1) \\
Einwohnerdichte & Einwohnerdichte & 2000 bis 2005 1) \\
$\varnothing$ LF/Betrieb & $\varnothing$ LF der landwirtschaftlichen Betriebe im Kreis & 2000 bis 2005 2) \\
Quote & Arbeitslosenquote & 2000 bis 2005 3) \\
Erreichbarkeit & $\varnothing$ PKW-Fahrzeit bis zum nächsten Oberzentrum & 2001 4) \\
EAD & Einwohner-Arbeitsplatz-Dichte & 2001 4) \\
Anteil ländliche & Anteil der Bevölkerung in ländlichen Gemeinden an der & $20014)$ \\
Bevölkerung & Gesamtbevölkerung & 2000 bis 2005 5) \\
Pacht (je ha) & $\varnothing$ Pachtpreis für Grünland/Ackerland (je ha) & 2000 bis 2005 5) 6) \\
Preisindex & Preisindex für landwirtschaftliche Produkte * 10-2 & 2000 bis 2005 5) \\
Dummyvariablen Region & &
\end{tabular}

1. ha $=$ Hektar, $\mathrm{LF}=$ Landwirtschaftlich genutzte Fläche, $\mathrm{EW}=$ Einwohner, Erw $=$ Erwerbstätige, $\mathrm{qkm}=$ Quadratkilometer

2. Regionale Merkmale beziehen sich auf die Merkmale von Kreisen und kreisfreien Städten (Nuts-III).

3. Der Preisindex für landwirtschaftliche Produkte ${ }^{77}$ nach Laspeyres wurde der Ebene der Kreise und kreisfreien Städte berechnet.

Quellen: 1) SÄBL (2004; 2005), 2) Destatis (div. Jgg.), 3) BAA (2005), 4) BBR (2003), 5) LAND-DATA GMBH (2006). 6) Testbetriebsnetz des BMVEL (2006).

77 Für die Berechnung des Erzeugerpreis-Index wurden die Preise und Umsatzerlöse folgender Produkte verwendet: Winterweizen, Sommerweizen, Hartweizen, Durum, Roggen, Wintergerste, Sommergerste, Hafer, Sommermenggetreide, Wintermenggetreide, Körnermais, CCM, Triticale, sonstiges Getreide, Ackerbohnen, Futtererbsen, sonstige Hülsenfrüchte zu Futterzwecken, sonstige Hülsenfrüchte, Winterraps, Sommerraps und Rübsen, Sonnenblumen, Sojabohnen, andere Ölsaaten (außer Öllein), Kartoffeln, Zuckerrüben, Milch, Ferkel, Eier. Wichtige Produktgruppen (z. B. Rindfleisch) fehlen, wenn nicht für jeden Kreis entsprechende Beobachtungen vorlagen. 
Tabelle A4: Deskriptive Statistik, Matching-Analyse

\begin{tabular}{|c|c|c|c|c|c|}
\hline \multirow[t]{2}{*}{ Variable } & \multirow[t]{2}{*}{ Einheit } & \multicolumn{2}{|c|}{ Agrarumweltmaßnahmen } & \multicolumn{2}{|c|}{ Ausgleichszulage } \\
\hline & & Mittelwert & $\begin{array}{l}\text { Standard- } \\
\text { abweichung }\end{array}$ & Mittelwert & $\begin{array}{l}\text { Standard- } \\
\text { abweichung }\end{array}$ \\
\hline \multicolumn{6}{|l|}{ Betriebliche Merkmale (2000) } \\
\hline $\ln L F$ & ha & 3,947 & 0,556 & 3,957 & 0,569 \\
\hline $\ln L F$ (quadriert) & ha & 15,885 & 4,509 & & \\
\hline ln Grünland & ha & 2,056 & 1,533 & 2,010 & 1,543 \\
\hline Anteil Grünland & $\%$ & & & 28,903 & 27,744 \\
\hline In Anteil Pachtland & $\%$ & 3,654 & 1,175 & 3,637 & 1,190 \\
\hline $\ln A K$ Betrieb & $\mathrm{AKE}$ & 0,372 & 0,436 & 0,371 & 0,442 \\
\hline $\ln$ AK Betrieb (je 100 ha) & $\mathrm{AKE}$ & 1,030 & 0,559 & 1,019 & 0,568 \\
\hline ln Außerbetriebliche AK & Stunden/Jahr & 1,179 & 2,698 & 1,204 & 2,719 \\
\hline $\ln$ Außerbetriebliche $A K B L$ & Stunden/Jahr & $-0,073$ & 0,298 & & \\
\hline ln Rinderbesatz & $\mathrm{GV}$ & 2,819 & 1,913 & 2,783 & 1,932 \\
\hline In Rinderbesatzdichte & GV & 0,463 & 0,530 & 0,464 & 0,535 \\
\hline ln Kapital (je ha) & TEuro & 2,390 & 0,769 & 2,394 & 0,777 \\
\hline ln Ausgaben Dünger (je ha) & TEuro & $-2,494$ & 0,648 & $-2,477$ & 0,622 \\
\hline ln Ausgaben PSM (je ha) & TEuro & $-2,703$ & 1,151 & $-2,685$ & 1,115 \\
\hline ln Viehbestand (je $100 \mathrm{ha}$ ) & VE & 4,565 & 1,629 & & \\
\hline ln Tierprämien (je ha) & TEuro & $-1,155$ & 1,700 & & \\
\hline ln Flächenprämien (je ha) & TEuro & $-1,408$ & 0,889 & $-1,397$ & 0,871 \\
\hline AZ-Zahlungen (je ha) & TEuro & 0,018 & 0,031 & & \\
\hline AUM-Teilnahme & $0=$ nein, $1=\mathrm{ja}$ & & & 0,021 & 0,143 \\
\hline AZ-Teilnahme & $0=$ nein, $1=\mathrm{ja}$ & 0,357 & 0,479 & & \\
\hline Ord. Ergebnis & Euro & 23.741 & 31.092 & 19.384 & 31.814 \\
\hline Ord. Ergebnis (je ha) & Euro & & & 429,337 & 591,854 \\
\hline Ord. Ergebnis $+P A / A K$ & Euro & 13.568 & 47.576 & & \\
\hline $\ln$ Umsatz & TEuro & 4,724 & 0,716 & 4,741 & 0,717 \\
\hline ln Umsatz (je ha) & TEuro & 0,779 & 0,643 & 0,785 & 0,637 \\
\hline Ldw. Bildung $($ Uni/FH) & $0=$ nein, $1=\mathrm{ja}$ & 0,275 & 0,446 & 0,276 & 0,447 \\
\hline Ldw. Bildung (Techniker) & $0=$ nein, $1=\mathrm{ja}$ & 0,015 & 0,120 & & \\
\hline Ldw. Bildung (Jahre) & Anzahl Jahre & 2,870 & 1,269 & & \\
\hline Nldw. Bildung (Uni/FH) & $0=$ nein, $1=\mathrm{ja}$ & 0,086 & 0,280 & 0,088 & 0,283 \\
\hline Ackerbau & $0=$ nein, $1=\mathrm{ja}$ & 0,189 & 0,391 & 0,209 & 0,407 \\
\hline Futterbau & $0=$ nein, $1=\mathrm{ja}$ & 0,465 & 0,499 & 0,458 & 0,498 \\
\hline Veredlung & $0=$ nein, $1=\mathrm{ja}$ & 0,135 & 0,342 & & \\
\hline Gemischt & $0=$ nein, $1=\mathrm{ja}$ & 0,076 & 0,265 & 0,073 & 0,261 \\
\hline$L V Z$ & Index & 35,550 & 13,528 & 36,065 & 13,804 \\
\hline Höhe & Höhe über N. N. & 285,009 & 221,337 & 273,006 & 216,874 \\
\hline
\end{tabular}


Tabelle A4: $\quad$ Deskriptive Statistik, Matching-Analyse - Fortsetzung

\begin{tabular}{|c|c|c|c|c|c|}
\hline \multirow[t]{2}{*}{ Variable } & \multirow[t]{2}{*}{ Einheit } & \multicolumn{2}{|c|}{ Agrarumweltmaßnahmen } & \multicolumn{2}{|c|}{ Ausgleichszulage } \\
\hline & & Mittelwert & $\begin{array}{l}\text { Standard- } \\
\text { abweichung }\end{array}$ & Mittelwert & $\begin{array}{l}\text { Standard- } \\
\text { abweichung }\end{array}$ \\
\hline \multicolumn{6}{|l|}{ Regionale Merkmale (2000) } \\
\hline Quote & $\%$ & 7,534 & 2,900 & & \\
\hline BWS Landwirtschaft & 1.000 Euro & 78,577 & 60,214 & 78,249 & 60,540 \\
\hline $\ln B I P$ (pro Kopf) & 1.000 Euro & 16,862 & 0,526 & 16,844 & 0,503 \\
\hline ln Einwohnerdichte & $\mathrm{EW} / \mathrm{qm}$ & 5,125 & 0,613 & 5,127 & 0,622 \\
\hline $\ln E A D$ & EW/Erw. & 5,377 & 0,620 & 5,373 & 0,626 \\
\hline Erreichbarkeit & Minuten & 32,466 & 12,950 & 32,418 & 13,218 \\
\hline Regland & Index & 36,495 & 24,311 & 36,482 & 24,593 \\
\hline ln Pacht & Euro/ha & & & 275,769 & 100,758 \\
\hline Anteil Ackerbau & $\%$ & 28,456 & 17,549 & 29,382 & 17,865 \\
\hline Anteil Futterbau & $\%$ & 42,715 & 19,603 & 42,651 & 19,473 \\
\hline Anteil Veredlung & $\%$ & 9,855 & 9,478 & 9,687 & 9,419 \\
\hline Anteil Gemischt & $\%$ & 6,796 & 3,835 & 6,599 & 3,762 \\
\hline Region Nord & $0=$ nein, $1=\mathrm{ja}$ & 0,236 & 0,425 & 0,257 & 0,437 \\
\hline Region West & $0=$ nein, $1=\mathrm{ja}$ & 0,286 & 0,452 & 0,297 & 0,457 \\
\hline Region Süd & $0=$ nein, $1=\mathrm{ja}$ & 0,463 & 0,499 & 0,427 & 0,495 \\
\hline Region Ost & $0=$ nein, $1=\mathrm{ja}$ & 0,016 & 0,125 & 0,019 & 0,137 \\
\hline \multicolumn{6}{|l|}{ Wachstumsraten (2000 bis 2005) } \\
\hline $\ln L F$ & $\%$ & 0,060 & 0,215 & 0,060 & 0,219 \\
\hline ln Grünland & $\%$ & $-0,009$ & 0,603 & $-0,013$ & 0,623 \\
\hline ln Anteil Pachtland & $\%$ & 0,005 & 0,855 & 0,003 & 0,871 \\
\hline $\ln A K$ Betrieb & $\%$ & $-0,011$ & 0,267 & $-0,013$ & 0,278 \\
\hline ln AK Betrieb (je 100 ha) & $\%$ & $-0,072$ & 0,317 & $-0,073$ & 0,327 \\
\hline $\ln$ Außerbetriebliche AK & $\%$ & 0,090 & 1,625 & 0,100 & 1,666 \\
\hline ln Kapital (je ha) & $\%$ & 0,025 & 0,489 & 0,029 & 0,494 \\
\hline ln Rinderbesatz & $\%$ & $-0,191$ & 0,780 & $-0,201$ & 0,794 \\
\hline In Rinderbesatzdichte & $\%$ & $-0,087$ & 0,471 & $-0,091$ & 0,479 \\
\hline ln Ausgaben PSM (je ha) & $\%$ & 0,062 & 1,217 & 0,056 & 1,188 \\
\hline ln Ausgaben Dünger (je ha) & $\%$ & 0,164 & 0,788 & 0,158 & 0,783 \\
\hline $\ln$ Umsatz & $\%$ & 0,063 & 0,467 & 0,055 & 0,479 \\
\hline ln Umsatz (je ha) & $\%$ & 0,006 & 0,426 & 0,000 & 0,432 \\
\hline Anzahl Beobachtungen & $\mathbf{n}$ & 19.337 & & 23.120 & \\
\hline
\end{tabular}

Quelle: eigene Berechnungen, Datenquellen vgl. Tabelle A2 und A3. 
Tabelle A5: $\quad$ Standardisierter Bias vor und nach dem Matching für die in der LogitAnalyse verwendeten erklärenden Variablen, Deutschland gesamt

\begin{tabular}{|c|c|c|c|c|c|c|c|}
\hline \multirow[t]{2}{*}{ Merkmal (2000) } & \multirow[t]{2}{*}{ Einheit } & \multicolumn{3}{|c|}{ Agrarumweltprogramme } & \multicolumn{3}{|c|}{ Ausgleichszulage } \\
\hline & & $\begin{array}{c}\text { SB vor } \\
\text { Matching }\end{array}$ & $\begin{array}{l}\text { SB nach } \\
\text { Matching }\end{array}$ & $\begin{array}{c}\text { SB reduziert } \\
\%\end{array}$ & $\begin{array}{c}\text { SB vor } \\
\text { Matching }\end{array}$ & $\begin{array}{l}\text { SB nach } \\
\text { Matching }\end{array}$ & $\begin{array}{c}\text { SB reduziert } \\
\%\end{array}$ \\
\hline \multicolumn{8}{|l|}{ Betriebliche Merkmale } \\
\hline $\ln L F$ & ha & $-17,79$ & 1,65 & $-109,25$ & & & \\
\hline $\ln L F$ (quadriert) & ha & & & & $-15,68$ & $-4,34$ & $-98,81$ \\
\hline ln Grünland & ha & $-33,78$ & 4,15 & $-112,50$ & & & \\
\hline In Anteil Grünland & $\%$ & & & & $-80,97$ & $-2,83$ & $-96,45$ \\
\hline In Anteil Pachtland & $\%$ & $-23,88$ & $-2,28$ & $-90,05$ & $-25,26$ & $-5,42$ & $-78,06$ \\
\hline $\ln$ AK Betrieb & AKE & $-33,31$ & 0,29 & $-100,89$ & $-22,78$ & $-2,14$ & $-91,02$ \\
\hline In Außerbetriebliche $A K$ & $\mathrm{~h} / \mathrm{Jahr}$ & 4,53 & 6,32 & 39,47 & & & \\
\hline In Außerbetriebliche $A K B L$ & $\mathrm{~h} / \mathrm{Jahr}$ & 7,41 & $-2,45$ & $-130,49$ & & & \\
\hline ln Rinderbesatz & $\mathrm{GV}$ & $-21,15$ & 6,60 & $-132,37$ & $-59,68$ & $-2,47$ & $-95,79$ \\
\hline ln Rinderbesatzdichte & GV & 0,20 & 2,50 & $1.130,42$ & $-9,95$ & $-0,46$ & $-95,09$ \\
\hline ln Kapital (je ha) & TEuro & $-6,37$ & $-2,88$ & $-52,38$ & & & \\
\hline ln Ausgaben Dünger (je ha) & TEuro & 20,17 & $-0,70$ & $-103,61$ & 34,37 & $-1,55$ & $-104,70$ \\
\hline ln Ausgaben PSM (je ha) & TEuro & $-4,59$ & 0,36 & $-107,88$ & 37,72 & 2,97 & $-92,07$ \\
\hline In Viehbestand (je 100 ha) & $\mathrm{VE}$ & 8,49 & 0,19 & $-97,56$ & & & \\
\hline ln Tierprämien (je ha) & TEuro & 3,16 & $-2,77$ & $-185,03$ & & & \\
\hline In Flächenprämien (je ha) & TEuro & $-1,94$ & 3,34 & $-271,66$ & $-4,67$ & 0,52 & $-111,04$ \\
\hline AZ-Zahlungen (je ha) & TEuro & $-78,47$ & $-5,76$ & $-92,79$ & & & \\
\hline AUM-Teilnahme & $0=$ nein, $1=\mathrm{ja}$ & & & & 7,24 & $-1,20$ & $-117,96$ \\
\hline AZ-Teilnahme & $0=$ nein, $1=\mathrm{ja}$ & $-96,29$ & $-4,72$ & $-95,14$ & & & \\
\hline Ord. Ergebnis & TEuro & $-12,19$ & 0,99 & $-107,70$ & & & \\
\hline Ord. Ergebnis (je ha) & TEuro & & & & $-1,22$ & 1,11 & $-195,38$ \\
\hline Ord. Ergebnis + PA/AK & TEuro & 0,04 & 1,40 & $3.631,23$ & $-12,22$ & 0,53 & $-103,73$ \\
\hline $\ln$ Umsatz & TEuro & 13,97 & $-1,60$ & $-112,22$ & 20,47 & $-2,67$ & $-112,97$ \\
\hline ln Umsatz (je ha) & TEuro & 31,49 & $-3,57$ & $-112,00$ & 39,33 & $-0,32$ & $-100,80$ \\
\hline Ldw. Bildung (Uni/FH) & $0=$ nein, $1=\mathrm{ja}$ & $-11,63$ & 2,71 & $-122,50$ & $-7,39$ & 0,78 & $-110,67$ \\
\hline Ldw. Bildung (Techniker) & $0=$ nein, $1=\mathrm{ja}$ & 0,78 & 1,57 & 118,43 & & & \\
\hline Ldw. Bildung (Jahre) & Anzahl Jahre & $-10,32$ & 3,89 & $-138,23$ & & & \\
\hline Nldw. Bildung (Uni/FH) & $0=$ nein, $1=\mathrm{ja}$ & 5,14 & 1,50 & $-71,45$ & 4,40 & 1,11 & $-76,00$ \\
\hline Futterbau & $0=$ nein, $1=\mathrm{ja}$ & 20,60 & 2,58 & $-86,33$ & 54,45 & 1,45 & $-97,56$ \\
\hline Ackerbau & $0=$ nein, $1=\mathrm{ja}$ & $-18,14$ & 3,71 & $-120,53$ & $-51,86$ & $-2,01$ & $-96,00$ \\
\hline Veredlung & $0=$ nein, $1=\mathrm{ja}$ & 18,90 & $-8,77$ & $-144,80$ & 25,64 & 0,36 & $-98,55$ \\
\hline Gemischt & $0=$ nein, $1=\mathrm{ja}$ & 5,64 & 2,42 & $-58,71$ & 10,13 & 0,00 & $-100,00$ \\
\hline$L V Z$ & Index & 18,75 & 0,45 & $-97,60$ & 117,67 & $-2,63$ & $-101,52$ \\
\hline Höhe & Höhe über N. N. & $-159,48$ & $-2,46$ & $-98,22$ & $-161,42$ & $-2,22$ & $-98,50$ \\
\hline
\end{tabular}


Tabelle A5: $\quad$ Standardisierter Bias vor und nach dem Matching für die in der LogitAnalyse verwendeten erklärenden Variablen, Deutschland gesamt Fortsetzung

\begin{tabular}{|c|c|c|c|c|c|c|c|}
\hline \multirow[t]{2}{*}{ Merkmal (2000) } & \multirow[t]{2}{*}{ Einheit } & \multicolumn{3}{|c|}{ Agrarumweltprogramme } & \multicolumn{3}{|c|}{ Ausgleichszulage } \\
\hline & & $\begin{array}{c}\text { SB vor } \\
\text { Matching }\end{array}$ & $\begin{array}{l}\text { SB nach } \\
\text { Matching }\end{array}$ & $\begin{array}{c}\text { SB reduziert } \\
\%\end{array}$ & $\begin{array}{c}\text { SB vor } \\
\text { Matching }\end{array}$ & $\begin{array}{l}\text { SB nach } \\
\text { Matching }\end{array}$ & $\begin{array}{c}\text { SB reduziert } \\
\%\end{array}$ \\
\hline \multicolumn{8}{|l|}{ Regionale Merkmale } \\
\hline Quote & $\%$ & 84,98 & 1,97 & $-97,29$ & 58,02 & 0,66 & $-98,81$ \\
\hline BWS Landwirtschaft & TEuro & 45,25 & $-1,54$ & $-103,65$ & 57,41 & 7,54 & $-88,70$ \\
\hline $\log$ BIP (pro Kopf) & TEuro & $-4,59$ & $-0,78$ & $-84,75$ & $-4,03$ & $-2,09$ & $-45,07$ \\
\hline ln Einwohnerdichte & $\mathrm{EW} / \mathrm{qm}$ & 14,20 & 3,25 & $-76,05$ & 61,84 & $-2,74$ & $-104,20$ \\
\hline $\ln E A D$ & EW/Erw. & 10,05 & 1,03 & $-89,55$ & 56,85 & $-2,07$ & $-103,42$ \\
\hline Erreichbarkeit & Minuten & 22,61 & $-4,16$ & $-119,03$ & $-1,44$ & 1,35 & $-188,81$ \\
\hline Regland & Index & $-30,80$ & $-3,14$ & $-89,61$ & $-66,82$ & 2,83 & $-103,76$ \\
\hline $\ln$ Pacht & Euro/ha & & & & 96,02 & 3,53 & $-96,40$ \\
\hline Anteil Futterbau & $\%$ & $-12,02$ & $-5,52$ & $-55,01$ & $-52,08$ & $-0,73$ & $-98,49$ \\
\hline Anteil Ackerbau & $\%$ & 13,83 & 3,39 & $-74,05$ & 48,92 & 0,01 & $-99,98$ \\
\hline Anteil Veredlung & $\%$ & 61,05 & $-2,07$ & $-103,74$ & 65,60 & 2,19 & $-96,59$ \\
\hline Anteil Gemischt & $\%$ & $-17,07$ & $-3,40$ & $-80,32$ & $-23,88$ & 1,20 & $-105,10$ \\
\hline \multicolumn{8}{|l|}{ Regionsdummies } \\
\hline Region Nord & $0=$ nein, $1=$ ja & 86,72 & 6,94 & $-90,00$ & 143,06 & $-1,93$ & $-100,47$ \\
\hline Region West & $0=$ nein, $1=\mathrm{ja}$ & 103,15 & $-4,21$ & $-105,07$ & 27,13 & 0,41 & $-98,52$ \\
\hline Region Süd & $0=$ nein, $1=\mathrm{ja}$ & $-223,08$ & $-2,71$ & $-98,42$ & & & \\
\hline Mittelwert & & $-4,90$ & $-0,05$ & 15,93 & 10,14 & $-0,31$ & $-102,05$ \\
\hline Median & & 0,49 & 0,32 & $-97,91$ & 5,81 & $-0,16$ & $-98,67$ \\
\hline
\end{tabular}

Der standardisierte Bias (SB) entspricht der Differenz der Mittelwerte zwischen Teilnehmer- und Kontrollbetrieben, geteilt durch die Quadratwurzel der mittleren Standardabweichung der Kovariate (Regressoren des Logit-Modells) von Teilnehmer- und Kontrollbetrieben * 100. Der SB kann als Bias, gemessen in Prozent der mittleren Standardabweichung, interpretiert werden (BASER, 2006).

Quelle: eigene Berechnungen, Datenquellen vgl. Tabelle A2 und A3. 
Tabelle A6: Effekte der AUM- und AZ-Teilnahme in Abhängigkeit von der Höhe der Programmzahlungen je Betrieb

\begin{tabular}{|c|c|c|c|c|c|c|c|}
\hline $\begin{array}{l}\text { Abhängige Variable } \\
\text { Unabhängige Variablen }\end{array}$ & $\begin{array}{l}\ln L F \\
\text { Einheit }\end{array}$ & $\begin{array}{c}\text { Heck (AUM) } \\
\text { Koeff. p }\end{array}$ & $\begin{array}{c}\text { Heck (AZ) } \\
\text { Koeff. p }\end{array}$ & $\begin{array}{l}\text { POLS II } \\
\text { Koeff. p }\end{array}$ & $\begin{array}{c}\text { FE II } \\
\text { Koeff. p }\end{array}$ & $\begin{array}{l}\text { GMM-OR } \\
\text { Koeff. p }\end{array}$ & $\begin{array}{l}\text { GMM-SYS } \\
\text { Koeff. p }\end{array}$ \\
\hline L. $\ln L F$ & ha & $\begin{array}{l}0,974 * * * \\
(0,005)\end{array}$ & $\begin{array}{l}0,965 * * * \\
(0,006)\end{array}$ & $\begin{array}{l}0,960 \text { *** } \\
(0,005)\end{array}$ & $\begin{array}{l}0,396 * * * \\
(0,006)\end{array}$ & $\begin{array}{l}0,805 * * * \\
(0,141)\end{array}$ & $\begin{array}{l}1,049 \text { *** } \\
(0,088)\end{array}$ \\
\hline L2. & ha & $\begin{array}{l}0,032 * * * \\
(0,005)\end{array}$ & $\begin{array}{l}0,035 * * * \\
(0,006)\end{array}$ & $\begin{array}{l}0,042 * * * \\
(0,005)\end{array}$ & $\begin{array}{l}-0,016 * * * \\
(0,005)\end{array}$ & $\begin{array}{r}0,181 \\
(0,136)\end{array}$ & $\begin{array}{r}-0,044 \\
(0,088)\end{array}$ \\
\hline $\ln$ Kapital & Euro & & & & & $\begin{array}{l}0,024 * * \\
(0,009)\end{array}$ & $\begin{array}{l}0,011 \text { *** } \\
(0,003)\end{array}$ \\
\hline L. & Euro & $\begin{array}{l}0,005 * * * \\
(0,001)\end{array}$ & $\begin{array}{l}0,007 * * * \\
(0,001)\end{array}$ & $\begin{array}{l}0,005 * * * \\
(0,000)\end{array}$ & $\begin{array}{l}0,006 * * * \\
(0,001)\end{array}$ & & \\
\hline $\ln A K$ Betrieb & AKE & & & & & $\begin{array}{l}0,035 \text { ** } \\
(0,017)\end{array}$ & $\begin{array}{r}0,006 \\
(0,004)\end{array}$ \\
\hline L. & AKE & $\begin{array}{l}0,007 \text { *** } \\
(0,001)\end{array}$ & $\begin{array}{l}0,008 \text { *** } \\
(0,001)\end{array}$ & $\begin{array}{l}0,007 \text { *** } \\
(0,001)\end{array}$ & $\begin{array}{l}0,009 \text { *** } \\
(0,002)\end{array}$ & & \\
\hline In Direktzahlungen & Euro & & & & & $\begin{array}{r}0,001 \\
(0,002)\end{array}$ & $\begin{array}{r}0,002 \\
(0,001)\end{array}$ \\
\hline L. $\sim$ & Euro & $\begin{array}{r}-0,0002 \\
(0,0002)\end{array}$ & $\begin{array}{r}0,0002 \\
(0,0002)\end{array}$ & $\begin{array}{l}0,0003 * \\
(0,002)\end{array}$ & $\begin{array}{r}-1 \mathrm{E}-06 \\
(0,0002)\end{array}$ & & \\
\hline Gewinn & 100T Euro & & & & & $\begin{array}{r}-0,018 \\
(0,011)\end{array}$ & $\begin{array}{r}0,001 \\
(0,010)\end{array}$ \\
\hline L. $\sim$ & 100T Euro & $\begin{array}{l}0,004 * * * \\
(0,001)\end{array}$ & $\begin{array}{l}0,004 * * * \\
(0,001)\end{array}$ & $\begin{array}{l}0,004 * * * \\
(0,001)\end{array}$ & $\begin{array}{l}-0,002 * * * \\
(0,001)\end{array}$ & & \\
\hline Alter Betriebsleiter & 10 Jahre & $\begin{array}{l}-0,003 * * * \\
(0,0003)\end{array}$ & $\begin{array}{l}-0,003 * * * \\
(0,0004)\end{array}$ & $\begin{array}{l}-0,004 \text { *** } \\
(0,0003)\end{array}$ & & & $\begin{array}{l}-0,004 * * * \\
(0,001)\end{array}$ \\
\hline In AUM-Zahlungen & Euro & $\begin{array}{l}0,002 * * * \\
(0,0004)\end{array}$ & & $\begin{array}{l}0,0005 * * * \\
(0,0001)\end{array}$ & $\begin{array}{c}0,001 * * * \\
(0,0002)\end{array}$ & $\begin{array}{l}0,002 * * \\
(0,001)\end{array}$ & $\begin{array}{l}0,0004 * * * \\
(0,0001)\end{array}$ \\
\hline ln AZ-Zahlungen & Euro & & $\begin{array}{r}0,001 \\
(0,001)\end{array}$ & $\begin{array}{l}0,00044^{* * *} \\
(0,0001)\end{array}$ & $\begin{array}{r}0,0001 \\
(0,0003)\end{array}$ & $\begin{array}{r}0,001 \\
(0,001)\end{array}$ & $\begin{array}{l}-0,0001 \\
(0,0003)\end{array}$ \\
\hline AUM-Teilnahme & $0=$ nein, $1=\mathrm{ja}$ & & $\begin{array}{r}0,002 \\
(0,001)\end{array}$ & & & & \\
\hline AZ-Teilnahme & $0=$ nein, $1=\mathrm{ja}$ & $\begin{array}{l}0,002 \text { ** } \\
(0,001)\end{array}$ & & & & & \\
\hline $\ln$ Pacht & 100 Euro/ha & $\begin{array}{l}-0,007 * \\
(0,004)\end{array}$ & $\begin{array}{l}-0,008 * \\
(0,004)\end{array}$ & $\begin{array}{r}-0,004 \\
(0,004)\end{array}$ & $\begin{array}{r}0,002 \\
(0,003)\end{array}$ & $\begin{array}{l}-0,004 \\
(0,005)\end{array}$ & $\begin{array}{l}-0,004 * * \\
(0,002)\end{array}$ \\
\hline Preisindex & $2.000=1 \%$ & $\begin{array}{l}-0,002 * \\
(0,001)\end{array}$ & $\begin{array}{l}-0,003 * \\
(0,002)\end{array}$ & $\begin{array}{l}-0,003 \text { *** } \\
(0,001)\end{array}$ & $\begin{array}{l}-0,003 * * * \\
(0,001)\end{array}$ & $\begin{array}{l}-0,002 * * \\
(0,001)\end{array}$ & $\begin{array}{l}-0,002 * * \\
(0,001)\end{array}$ \\
\hline $\ln \varnothing L F /$ Betrieb & ha & $\begin{array}{r}0,003 \\
(0,017)\end{array}$ & $\begin{array}{l}-0,045 * * \\
(0,019)\end{array}$ & $\begin{array}{l}0,021 * * \\
(0,011)\end{array}$ & $\begin{array}{l}0,046 \text { *** } \\
(0,010)\end{array}$ & $\begin{array}{l}0,032 * * \\
(0,013)\end{array}$ & $\begin{array}{l}-0,005 * \\
(0,002)\end{array}$ \\
\hline Konstante & & $\begin{array}{l}-0,162 * \\
(0,085)\end{array}$ & $\begin{array}{r}0,138 \\
(0,102)\end{array}$ & $\begin{array}{l}-0,094 * * \\
(0,043)\end{array}$ & $\begin{array}{l}2,238 * * * \\
(0,051)\end{array}$ & & $\begin{array}{l}-0,121 * * * \\
(0,039)\end{array}$ \\
\hline Dummy Betriebsform & & & & ja & & & ja \\
\hline $\begin{array}{l}\text { Dummy Jahr } \\
\text { Dummy Kreis }\end{array}$ & & & & ja & ja & ja & ja \\
\hline
\end{tabular}


Tabelle A6: $\quad$ Effekte der AUM- und AZ-Teilnahme in Abhängigkeit von der Höhe der Programmzahlungen je Betrieb - Fortsetzung

\begin{tabular}{|c|c|c|c|c|c|c|c|}
\hline \multicolumn{2}{|c|}{ Selektionsgleichung ( $1^{\text {st }}$ stage $)$} & \multirow{2}{*}{$\begin{array}{c}\mathrm{AUM}=1 \\
0,454 * * * \\
(0,009)\end{array}$} & \multicolumn{2}{|l|}{$\mathrm{AZ}=1$} & & & \\
\hline L. $L F$ & ha & & & & & & \\
\hline L. Anteil Grünland & ha & $\begin{array}{l}0,004 * * * \\
(0,000)\end{array}$ & $\begin{array}{l}0,014 * * * \\
(0,000)\end{array}$ & & & & \\
\hline$R G V$ (minmax) & $0=$ nein, $1=\mathrm{ja}$ & $\begin{array}{l}0,418 * * * \\
(0,016)\end{array}$ & & & & & \\
\hline Ausgaben Dünger (je ha) & TEuro & $\begin{array}{l}-1,432 * * * \\
(0,112)\end{array}$ & & & & & \\
\hline Ausgaben PSM (je ha) & TEuro & $\begin{array}{l}0,514 * * * \\
(0,125)\end{array}$ & & & & & \\
\hline$B W S L d w$ & Euro/ha & & $\begin{array}{l}-0,291 * * * \\
(0,007)\end{array}$ & & & & \\
\hline$L V Z$ & Index & $\begin{array}{l}-0,007 * * * \\
(0,001)\end{array}$ & $\begin{array}{l}-0,168 * * * \\
(0,001)\end{array}$ & & & & \\
\hline Konstante & & $\begin{array}{l}-3,871 * * * \\
(0,185)\end{array}$ & $-1,231$ & & & & \\
\hline Dummy Betriebsform & & ja & ja & & & & \\
\hline Dummy Land & & ja & ja & & & & \\
\hline Lambda & & $\begin{array}{l}0,022 * * * \\
(0,003)\end{array}$ & $\begin{array}{l}-0,006 * * * \\
(0,001)\end{array}$ & & & & \\
\hline Beobachtungen & $N$ & 90.204 & 90.204 & 90.204 & 90.204 & 67.653 & 90.204 \\
\hline Selektierte Beobachtungen & & 47.099 & 32.644 & 90.204 & 90.204 & 67.653 & 90.204 \\
\hline Betriebe & $i$ & 22.551 & 22.551 & 22.551 & 22.551 & 22.551 & 22.551 \\
\hline Zeitpunkte & $T$ & 4 & 4 & 4 & 4 & 3 & 4 \\
\hline Instrumente & $n$ & & & & & 20 & 36 \\
\hline $\mathrm{R}^{2}$ & & & & 0,98 & 0,20 & & \\
\hline F Test, Wald $\left(\mathrm{Chi}^{2}\right)$ & & $2.910 .000 * * *$ & $2.020 .000 * * *$ & $218.662 * * *$ & $520.4 * * *$ & $3.741 * * *$ & $1.130 .000 * * *$ \\
\hline Hansen $J$ Test & & & & & & 7,02 & 17,33 \\
\hline AR(1) Test & & & & $-0,75$ & & $-2,97 * * *$ & $-6,37 * * *$ \\
\hline AR(2) Test & & & & 0,95 & & $-0,92$ & 1,11 \\
\hline
\end{tabular}

1. L. $=$ Wert des Vorjahres $(t-1)$. L2. $=$ Wert für $t-2$.

2. Gegenüber Heteroskedastizität und Autokorrelation robuste Standardfehler in Klammern.

3. Signifikanzniveau: $* * * \mathrm{p}<0,01, * * \mathrm{p}<0,05, * \mathrm{p}<0,1$.

4. $\mathrm{H}_{0}$ Hansen $J$ Test: Verwendete Instrumente sind gemeinsam valide.

5. $\mathrm{H}_{0} \mathrm{AR}(1)$-Test, AR(2)-Test: Keine Autokorrelation erster und zweiter Ordnung.

6. Zweistufige GMM-Schätzungen, Korrektur der Standardfehler nach WINDMEIJER (2000), gestauchte Instrumentenmatrix.

7. Verwendete Instrumente GMM-OR und GMM-SYS (transformierte Gleichung): $\ln L F_{t-3 / 4}, \ln K a p_{t-2 / 3}$, ln $D Z_{t-2 / 3}, \ln A K_{t-2 / 3}, \ln$ Gew $_{t-2 / 3}, A U M_{t-1 / 2}, A Z_{t-1 / 2}, \ln$ Pacht $_{t}$, Preis $_{t}, \ln M L F_{t}, \mu_{t}$, nur GMM-SYS: Alter,$\omega_{f}$

8. Verwendete Instrumente GMM-SYS (untransformierte Gleichung): $\ln L F_{t-2}, \ln K a p_{t-2}, \ln D Z_{t-2}, \ln A K_{t-2}$,

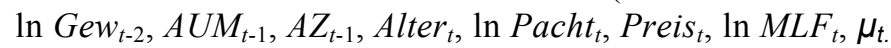

Quelle: eigene Berechnungen, Datenquellen vgl. Tabelle A2 und A3. 
Tabelle A7: Effekt der Ausgleichszulage auf das Flächenwachstum in Abhängigkeit vom Anteil der betrieblichen LF im benachteiligten Gebiet

\begin{tabular}{|c|c|c|c|c|c|c|}
\hline Abhängige Variable & $\ln L F$ & POLS II & & FE II & GMM-OR & GMM-SYS \\
\hline Unabhängige Variablen & Einheit & Koeff. $p^{1)}$ & $\mathrm{p}^{2)}$ & Koeff. $\mathrm{p}^{1)} \mathrm{p}^{2)}$ & Koeff. $\mathrm{p}^{1)} \mathrm{p}^{2)}$ & Koeff. $p^{1)} p^{2)}$ \\
\hline \multicolumn{7}{|c|}{ AUM-Teilnehmer nach Anteil der LF im benachteiligten Gebiet } \\
\hline $25 \%$ bis $50 \%$ der $L F$ & $0=$ nein, $1=\mathrm{ja}$ & 0,002 & & 0,007 & 0,021 & $0,010 *$ \\
\hline $50 \%$ bis $100 \%$ der $L F$ & $0=$ nein, $1=\mathrm{ja}$ & 0,003 & & 0,004 & $-0,006$ & 0,003 \\
\hline 3) $100 \% \operatorname{der} L F$ & $0=$ nein, $1=\mathrm{ja}$ & $0,004 * * *$ & & $0,005 * *$ & $0,015 * *$ & $0,006 *$ \\
\hline \multicolumn{7}{|c|}{ AZ-Teilnehmer nach Anteil der LF im benachteiligten Gebiet } \\
\hline $25 \%$ bis $50 \%$ der $L F$ & $0=$ nein, $1=\mathrm{ja}$ & 0,001 & & 0,001 & $-0,003$ & $-0,0003$ \\
\hline $50 \%$ bis $100 \% \operatorname{der} L F$ & $0=$ nein, $1=\mathrm{ja}$ & 0,001 & & 0,008 & $-0,004$ & 0,014 \\
\hline 3) $100 \% \operatorname{der} L F$ & $0=$ nein, $1=\mathrm{ja}$ & $0,003 *$ & & $-0,005$ & $0,019 *$ & $-0,003$ \\
\hline
\end{tabular}

1. Gegenüber Heteroskedastizität und Autokorrelation robuste Standardfehler in Klammern.

2. Signifikanzniveau: $* * * \mathrm{p}<0,01, * * \mathrm{p}<0,05, * \mathrm{p}<0,1$.

4. Nullhypothesen des Wald Tests: ${ }^{1)}$ Effekt der Teilnahme ist Null. ${ }^{2)}$ Effekt ist im Vergleich zu Teilnehmern mit $25 \%$ bis $50 \%$ der LF gleich. ${ }^{3)}$ Effekt der Teilnahme ist für alle Teilnehmer gleich.

Quelle: eigene Berechnungen, Datenquellen vgl. Tabelle A2 und A3. 
Tabelle A8: Probit-Modelle zur Schätzung der Wahrscheinlichkeit der AUMTeilnahme

\begin{tabular}{|c|c|c|c|c|c|c|}
\hline Abhängige Variable & $A U M(0,1)$ & 2001 & 2002 & 2003 & 2004 & 2005 \\
\hline Unabhängige Variablen & Einheit & Koeff. p & Koeff. $\mathrm{p}$ & Koeff. p & Koeff. p & Koeff. p \\
\hline $\ln L . L F$ & ha & $\begin{array}{l}0,389 * * * \\
(0,038)\end{array}$ & $\begin{array}{l}0,403 \text { *** } \\
(0,038)\end{array}$ & $\begin{array}{l}0,424 * * * \\
(0,037)\end{array}$ & $\begin{array}{l}0,431 \\
(0,035)\end{array}$ & $\begin{array}{l}0,506 \text { *** } \\
(0,034)\end{array}$ \\
\hline L. Anteil Grünland & $\%$ & $\begin{array}{r}0,002 \\
(0,001)\end{array}$ & $\begin{array}{r}0,002 \\
(0,001)\end{array}$ & $\begin{array}{l}0,004 * * \\
(0,002)\end{array}$ & $\begin{array}{l}0,003 \\
(0,001)\end{array}$ & $\begin{array}{l}0,005 \text { ** } \\
(0,001)\end{array}$ \\
\hline L. Ausgaben Dünger (je ha) & TEuro & $\begin{array}{l}-2,637 * * * \\
(0,505)\end{array}$ & $\begin{array}{l}-2,249 * * * \\
(0,421)\end{array}$ & $\begin{array}{l}-2,372 * * * \\
(0,431)\end{array}$ & $\begin{array}{l}-2,047 * * * \\
(0,422)\end{array}$ & $\begin{array}{l}-1,979 \text { *** } \\
(0,471)\end{array}$ \\
\hline L. Ausgaben PSM (je ha) & TEuro & $\begin{array}{l}1,062 * * \\
(0,532)\end{array}$ & $\begin{array}{r}0,807 \\
(0,532)\end{array}$ & $\begin{array}{r}0,489 \\
(0,494)\end{array}$ & $\begin{array}{r}0,248 \\
(0,495)\end{array}$ & $\begin{array}{r}0,741 \\
(0,468)\end{array}$ \\
\hline$R G V / H F F$ (minmax) & $0=$ nein, $1=\mathrm{ja}$ & $\begin{array}{l}0,333 * * * \\
(0,055)\end{array}$ & $\begin{array}{l}0,404 \text { *** } \\
(0,055)\end{array}$ & $\begin{array}{l}0,400 * * * \\
(0,060)\end{array}$ & $\begin{array}{l}0,450 * * * \\
(0,049)\end{array}$ & $\begin{array}{l}0,422 \text { *** } \\
(0,051)\end{array}$ \\
\hline$L V Z$ & Index & $\begin{array}{l}-0,014 * * * \\
(0,004)\end{array}$ & $\begin{array}{l}-0,012 \text { *** } \\
(0,004)\end{array}$ & $\begin{array}{l}-0,010 * * * \\
(0,004)\end{array}$ & $\begin{array}{l}-0,006 * \\
(0,004)\end{array}$ & $\begin{array}{l}-0,003 \\
(0,004)\end{array}$ \\
\hline Konstante & & $\begin{array}{l}-8,008 * * * \\
(0,334)\end{array}$ & $\begin{array}{l}-3,603 * * * \\
(0,380)\end{array}$ & $\begin{array}{l}-3,577 \text { *** } \\
(0,574)\end{array}$ & $\begin{array}{l}-3,437 \text { *** } \\
(0,197)\end{array}$ & $\begin{array}{l}-3,891 * * * \\
(0,196)\end{array}$ \\
\hline Dummy Betriebsform & & ja & ja & ja & ja & ja \\
\hline Dummy Land & & ja & ja & ja & ja & ja \\
\hline Beobachtungen & & 22.551 & 22.551 & 22.551 & 22.551 & 22.551 \\
\hline $\mathrm{R}^{2}$ & & 0,41 & 0,36 & 0,35 & 0,32 & 0,31 \\
\hline Log Likelihood & & -9.247 & -9.964 & -10.186 & -10.553 & -10.768 \\
\hline
\end{tabular}

1. L. $=$ Wert des Vorjahres $(t-1)$.

2. Gegenüber Heteroskedastizität und Autokorrelation robuste Standardfehler in Klammern.

3. Signifikanzniveau: $* * * \mathrm{p}<0,01, * * \mathrm{p}<0,05, * \mathrm{p}<0,1$.

Quelle: eigene Berechnungen, Datenquellen vgl. Tabelle A2 und A3. 
Tabelle A9: Probit-Modelle zur Schätzung der Wahrscheinlichkeit der AZ-Teilnahme

\begin{tabular}{|c|c|c|c|c|c|c|}
\hline Abhängige Variable & $A Z(0,1)$ & 2001 & 2002 & 2003 & 2004 & 2005 \\
\hline Unabhängige Variablen & Einheit & Koeff. $\mathrm{p}$ & Koeff. $\mathrm{p}$ & Koeff. p & Koeff. p & Koeff. $\mathrm{p}$ \\
\hline L. Anteil Grünland & ha & $\begin{array}{c}0,012 * * * \\
(0,002)\end{array}$ & $\begin{array}{l}0,013 * * * \\
(0,002)\end{array}$ & $\begin{array}{c}0,014 \text { *** } \\
(0,002)\end{array}$ & $\begin{array}{l}0,014 * * * \\
(0,002)\end{array}$ & $\begin{array}{l}0,014 * * * \\
(0,002)\end{array}$ \\
\hline $\ln B W S L d w$. (je ha) & Euro & $\begin{array}{l}-0,242 * * * \\
(0,073)\end{array}$ & $\begin{array}{l}-0,259 * * * \\
(0,075)\end{array}$ & $\begin{array}{l}-0,272 \text { *** } \\
(0,071)\end{array}$ & $\begin{array}{l}-0,317 * * * \\
(0,073)\end{array}$ & $\begin{array}{l}-0,304 * * * \\
(0,071)\end{array}$ \\
\hline$L V Z$ & Index & $\begin{array}{l}-0,191 * * * \\
(0,012)\end{array}$ & $\begin{array}{l}-0,182 * * * \\
(0,013)\end{array}$ & $\begin{array}{l}-0,167 * * * \\
(0,010)\end{array}$ & $\begin{array}{l}-0,164 * * * \\
(0,010)\end{array}$ & $\begin{array}{l}-0,159 * * * \\
(0,009)\end{array}$ \\
\hline Konstante & & $\begin{array}{r}-1,700 \\
(0,000)\end{array}$ & $\begin{array}{r}-1,553 \\
(0,000)\end{array}$ & $\begin{array}{r}-1,429 \\
(0,000)\end{array}$ & $\begin{array}{r}-0,912 \\
(0,000)\end{array}$ & $\begin{array}{r}-1,243 \\
(0,000)\end{array}$ \\
\hline Dummy Betriebsform & & ja & ja & ja & ja & ja \\
\hline Dummy Land & & ja & ja & ja & ja & ja \\
\hline Beobachtungen & $\mathrm{N}$ & 22.551 & 22.551 & 22.551 & 22.551 & 22.551 \\
\hline $\mathrm{R}^{2}$ & & 0,55 & 0,56 & 0,54 & 0,54 & 0,53 \\
\hline Log Likelihood & & -6.587 & -6.539 & -6.743 & -6.810 & -6.877 \\
\hline
\end{tabular}

1. L. $=$ Wert des Vorjahres $(t-1)$.

2. Gegenüber Heteroskedastizität und Autokorrelation robuste Standardfehler in Klammern.

3. Signifikanzniveau: $* * * \mathrm{p}<0,01, * * \mathrm{p}<0,05, * \mathrm{p}<0,1$.

Quelle: eigene Berechnungen, Datenquellen vgl. Tabelle A2 und A3. 
Tabelle A10: Zusammenhang zwischen dem Alter des Betriebsleiters und der Teilnahme an Agrarumweltmaßnahmen, $\mathrm{Chi}^{2}-$ Test

\begin{tabular}{|c|c|c|c|}
\hline \multirow[t]{2}{*}{ Alter Betriebsleiter } & \multicolumn{3}{|c|}{ Agrarumweltmaßnahmen } \\
\hline & Nichtteilnehmer & Teilnehmer & Summe \\
\hline \multicolumn{4}{|l|}{ unter 45 Jahre } \\
\hline Häufigkeit & 29.765 & 20.008 & 49.773 \\
\hline Zeilenprozent & 59,8 & 40,2 & 100,0 \\
\hline Spaltenprozent & 39,0 & 33,9 & 36,8 \\
\hline Beitrag zu Chi ${ }^{2}$ & 104,6 & 135,1 & 239,7 \\
\hline \multicolumn{4}{|l|}{45 bis 60 Jahre } \\
\hline Häufigkeit & 26.508 & 20.739 & 47.247 \\
\hline Zeilenprozent & 56,1 & 43,9 & 100,0 \\
\hline Spaltenprozent & 34,8 & 35,1 & 34,9 \\
\hline Beitrag zu Chi ${ }^{2}$ & 1,8 & 2,3 & 4,0 \\
\hline \multicolumn{4}{|l|}{ über 60 Jahre } \\
\hline Häufigkeit & 19.985 & 18.301 & 38.286 \\
\hline Zeilenprozent & 52,2 & 47,8 & 100,0 \\
\hline Spaltenprozent & 26,2 & 31,0 & 28,3 \\
\hline Beitrag zu Chi ${ }^{2}$ & 168,7 & 217,9 & 386,6 \\
\hline \multicolumn{4}{|l|}{ Summe } \\
\hline Häufigkeit & 76.258 & 59.048 & 135.306 \\
\hline Zeilenprozent & 56,4 & 43,6 & 100,0 \\
\hline Spaltenprozent & 100,0 & 100,0 & 100,0 \\
\hline Beitrag zu Chi ${ }^{2}$ & 275,1 & 355,3 & $630,3 * * *$ \\
\hline
\end{tabular}

1. Nullhypothese $\mathrm{Chi}^{2}$ - Tests: Die Werte in den Spalten und Zeilen sind unabhängig voneinander verteilt. 2. Signifikanzniveau: ${ }^{* * *} \mathrm{p}<0,01,{ }^{* *} \mathrm{p}<0,05,{ }^{*} \mathrm{p}<0,1$. Fett: Pearson $\mathrm{Chi}^{2}$.

Quelle: eigene Berechnungen, Datenquellen vgl. Tabelle A2 und A3. 
Tabelle A11: Effekt der AUM-Teilnahme auf das Flächenwachstum (vor und nach 2003) in Abhängigkeit von der Betriebsform

\begin{tabular}{|c|c|c|c|c|c|}
\hline Abhängige Variable & $\log L F$ & POLS II & FE II & GMM-DIFF & GMM-SYS \\
\hline Unabhängige Variablen & Einheit & Koeff. $p^{1)} p^{2)}$ & Koeff. $\mathrm{p}^{1)} \mathrm{p}^{2)}$ & Koeff. $p^{1)} p^{2)}$ & Koeff. $p^{1)} p^{2)}$ \\
\hline \multicolumn{6}{|c|}{ AUM-Teilnehmer der Betriebsform (Untersuchungszeitraum 2000 bis 2003) } \\
\hline Ackerbau & $0=$ nein, $1=\mathrm{ja}$ & $0,004 * *$ & 0,005 & 0,004 & 0,007 \\
\hline Futterbau & $0=$ nein, $1=\mathrm{ja}$ & 0,002 & $-0,001$ & 0,004 & 0,000 \\
\hline Veredlung & $0=$ nein, $1=\mathrm{ja}$ & 0,001 & $-0,003$ & 0,003 & 0,001 \\
\hline $\begin{array}{l}\text { Gemischt } \\
\text { 3) }\end{array}$ & $0=$ nein, $1=\mathrm{ja}$ & $-0,001$ & $-0,005$ & 0,033 & 0,009 \\
\hline \multicolumn{6}{|c|}{ AUM-Teilnehmer der Betriebsform (Untersuchungszeitraum 2004 bis 2005) } \\
\hline Ackerbau & $0=$ nein, $1=\mathrm{ja}$ & $0,005 * * *$ & $0,010 * * *$ & $-0,005$ & 0,007 \\
\hline Futterbau & $0=$ nein, $1=\mathrm{ja}$ & $0,003 * * *$ & 0,003 & $0,016 * * *$ & $0,011 * * *$ \\
\hline Veredlung & $0=$ nein, $1=\mathrm{ja}$ & 0,003 & $0,010 *$ & 0,002 & 0,002 \\
\hline Gemischt & $0=$ nein, $1=\mathrm{ja}$ & 0,001 & 0,001 & $-0,007$ & $-0,004$ \\
\hline
\end{tabular}

1. Gegenüber Heteroskedastizität und Autokorrelation robuste Standardfehler in Klammern.

2. Signifikanzniveau: $* * * \mathrm{p}<0,01, * * \mathrm{p}<0,05, * \mathrm{p}<0,1$.

3. Nullhypothesen des Wald Tests: ${ }^{1)}$ Effekt der Teilnahme ist Null. ${ }^{2)}$ Effekt ist im Vergleich zu Teilnehmern mit Ackerbau gleich. ${ }^{3)}$ Effekt der Teilnahme ist für alle Teilnehmer gleich.

Quelle: eigene Berechnungen, Datenquellen vgl. Tabelle A2 und A3. 


\section{Anhang}

Abbildungen 


\section{Verzeichnis der Abbildungen im Anhang}

Abbildung A1: Durchschnittlich gezahlte Ausgleichszulage je Hektar LF, Grünland (GL) und Acker (AL) im Jahr 2001, aufgeschlüsselt nach Bundesländern

Abbildung A2: $\quad$ Einfluss der bedingten Wahrscheinlichkeit der AUMTeilnahme $p(X)$ auf die Ergebnisänderung $\Delta y_{i}^{1}, \Delta y_{j}^{0}$ (nicht-parametrische Regression), Deutschland gesamt

Abbildung A3: $\quad$ Einfluss der bedingten Wahrscheinlichkeit der AZTeilnahme $p(X)$ auf die Ergebnisänderung $\Delta y_{i}^{1}, \Delta y_{j}^{0}$ (nicht-parametrische Regression), Deutschland gesamt

Abbildung A4: $\quad$ Mittelwerte der beobachteten (logarithmierten) LF und der LF-Wachstumsrate (in \%) für alle in der Panelanalyse berücksichtigten Betriebe $(n=22.551)$

Abbildung A5: $\quad$ Mittelwerte der beobachteten (logarithmierten) LF und der LF-Wachstumsrate (in \%) für alle in der Panelanalyse berücksichtigten Betriebe $(\mathrm{n}=22.551)$, differenziert nach AUM-Teilnehmern und AUM-Nichtteilnehmern

Abbildung A6: Mittelwerte der beobachteten (logarithmierten) LF und der LF-Wachstumsrate (in \%) für alle in der Panelanalyse berücksichtigten Betriebe $(\mathrm{n}=22.551)$, differenziert nach AZ-Teilnehmern und AZ-Nichtteilnehmern

Abbildung A7: $\quad$ Verteilung der AUM- und AZ-Teilnehmer nach der bedingten Wahrscheinlichkeit der Programmteilnahme 
Abbildung A1: $\quad$ Durchschnittlich gezahlte Ausgleichszulage je Hektar LF, Grünland (GL) und Acker (AL) im Jahr 2001, aufgeschlüsselt nach Bundesländern

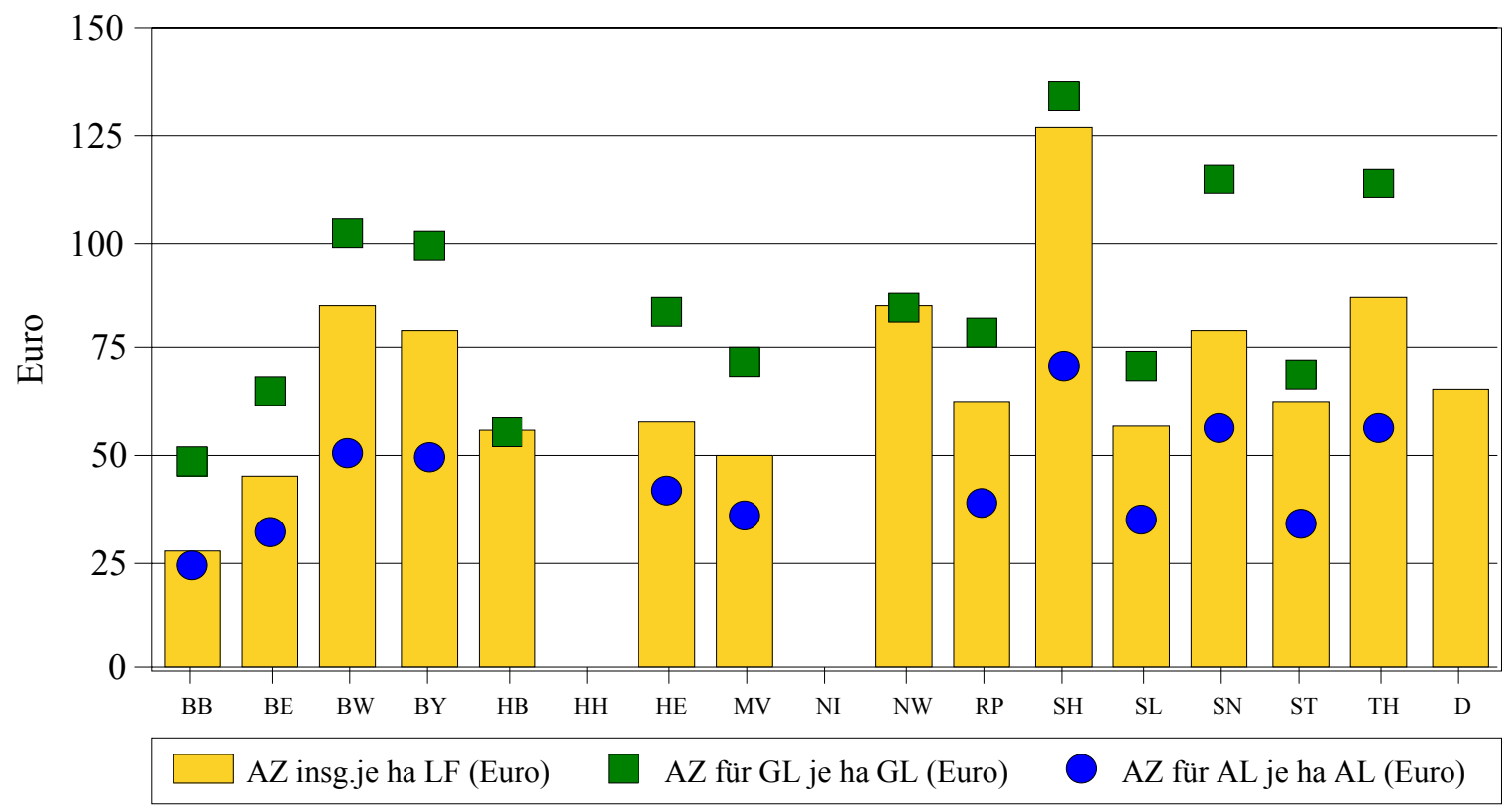

$\mathrm{BB}=$ Brandenburg, $\mathrm{BE}=$ Berlin, $\mathrm{BW}=$ Baden-Württemberg, $\mathrm{BY}=$ Bayern, $\mathrm{HB}=$ Hansestadt Bremen, $\mathrm{HH}=$ Hansestadt Hamburg, $\mathrm{HE}=$ Hessen, $\mathrm{MV}=$ Mecklenburg-Vorpommern, NI = Niedersachsen, $\mathrm{NW}=$ Nordrhein-Westfalen, $\mathrm{RP}=$ Rheinland-Pfalz, $\mathrm{SH}=$ Schleswig-Holstein, $\mathrm{SL}=\mathrm{Saarland}$, $\mathrm{SN}=$ Sachsen, $\mathrm{ST}=$ Sachsen-Anhalt, $\mathrm{TH}=$ Thüringen, $\mathrm{D}=$ Deutschland

Quelle: BERNHARDS et al. (2003). 
Abbildung A2: $\quad$ Einfluss der bedingten Wahrscheinlichkeit der AUM-Teilnahme $p(X)$ auf die Ergebnisänderung $\Delta y_{i}^{1}, \Delta y_{j}^{0} \quad$ (nicht-parametrische Regression), Deutschland gesamt

In Anteil Pachtland

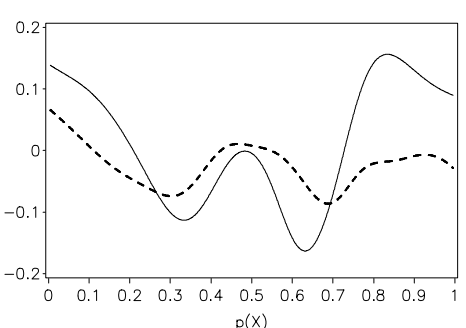

In Kapital (je ha)

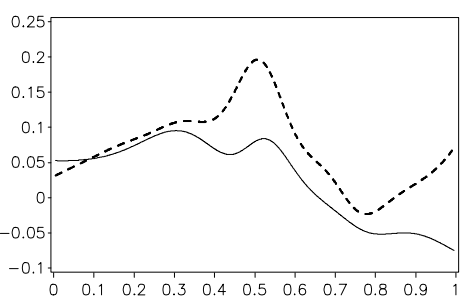

$p(x)$

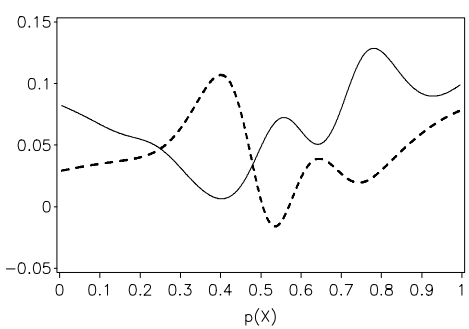

In AK Betrieb

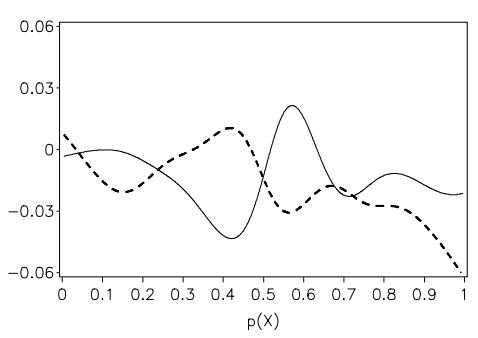

In RGV

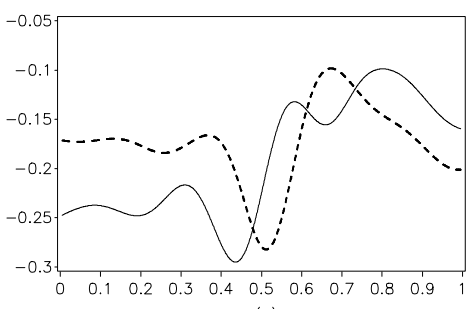

Kerndichte

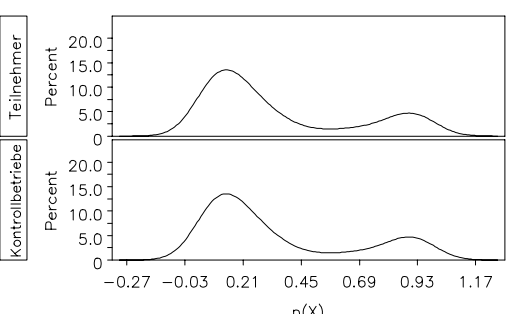

$p(x)$
In ausserbetriebliche Arbeit

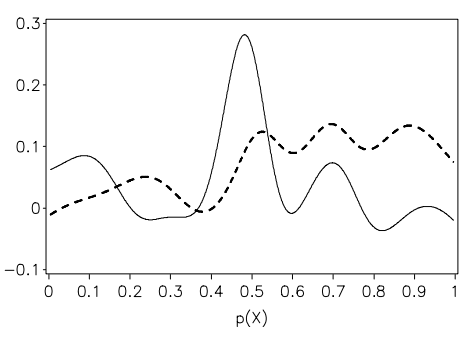

In PSM (je ha)

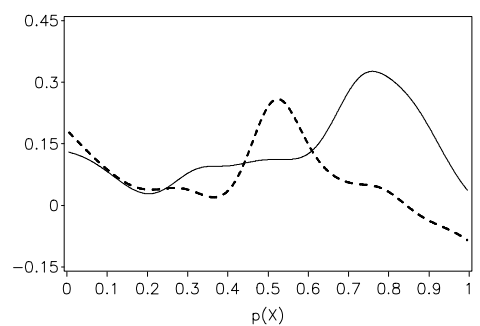

$p(x)$

1. Nadaraya-Watson Schätzer mit Gauß'scher Kernfunktion und optimaler Bandbreite.

2. In den Plots ist die Ergebnisänderung $\left(\Delta y_{i}^{1}, \Delta y_{j}^{0}\right)$ von Teilnehmern (durchgezogene Linie) und Kontrollbetrieben (gestrichelte Linie) in Abhängigkeit von der Wahrscheinlichkeit der AUM-Teilnahme $(p(X))$ dargestellt. Der Effekt der Programmteilnahme auf die Teilnehmer (ATT) entspricht der Fläche zwischen beiden Linien.

Quelle: eigene Berechnungen, Datenquellen vgl. Tabelle A2 und A3. 
Abbildung A3: $\quad$ Einfluss der bedingten Wahrscheinlichkeit der AZ-Teilnahme $p(X)$ auf die Ergebnisänderung $\Delta y_{i}^{1}, \Delta y_{j}^{0}$ (nicht-parametrische Regression), Deutschland gesamt

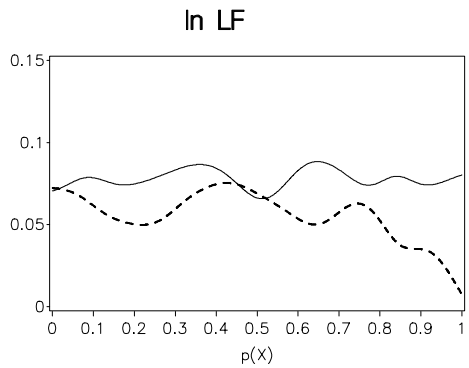

In AK Betrieb

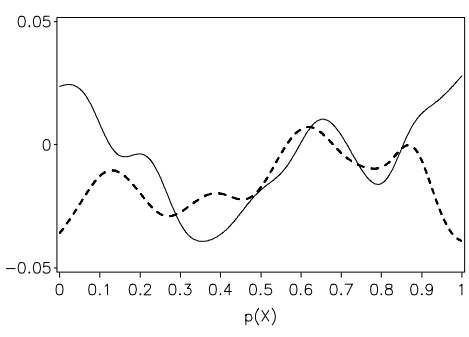

In Kapital (je ha)

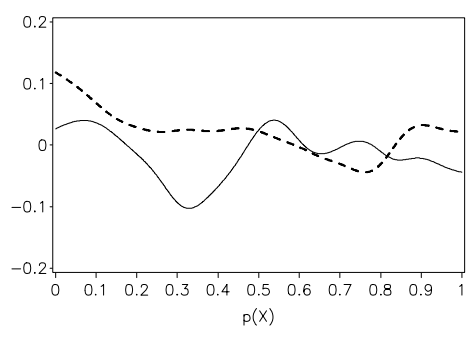

In PSM (je ha)

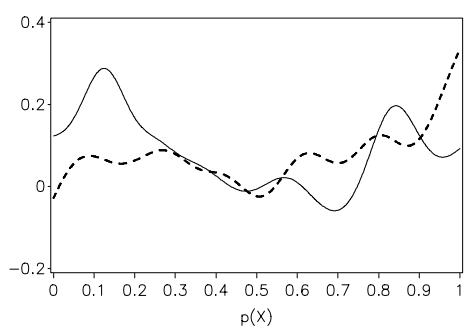

In Umsatz (je ha)

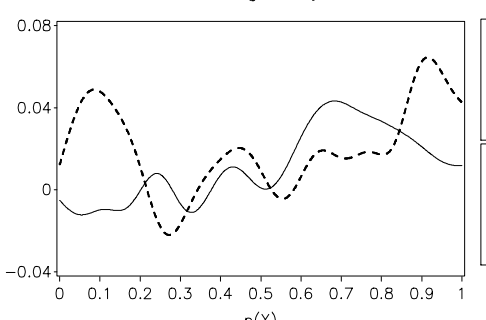

In Gruenland

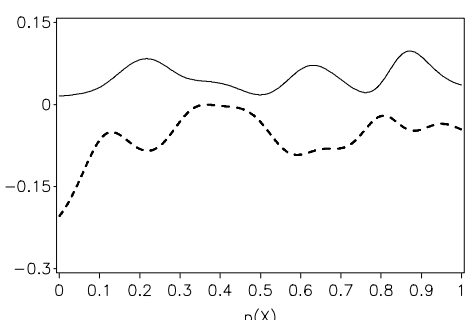

In AK Betrieb (je 100 ha)

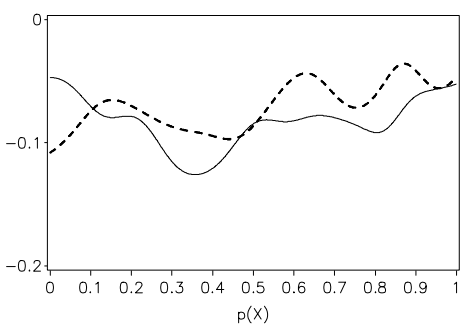

In RGV

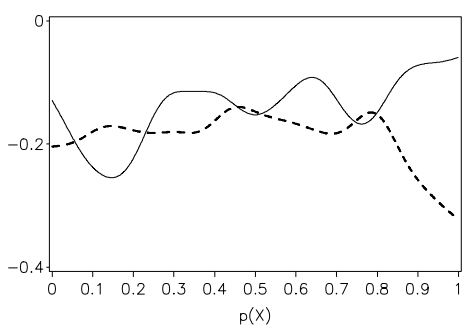

In Duenger (je ha)

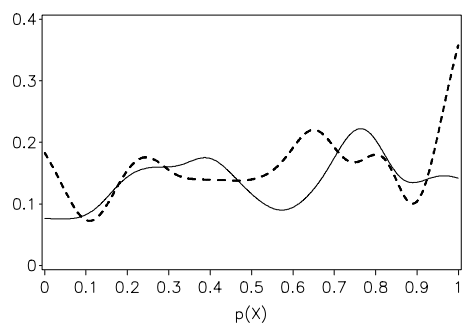

Kerndichte

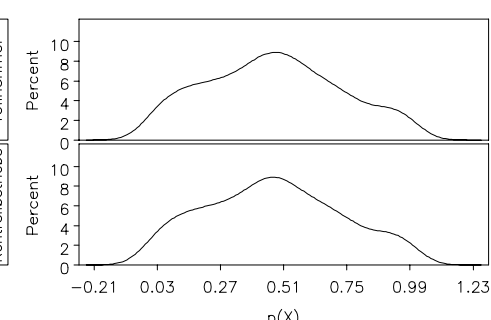

In Anteil Pachtland

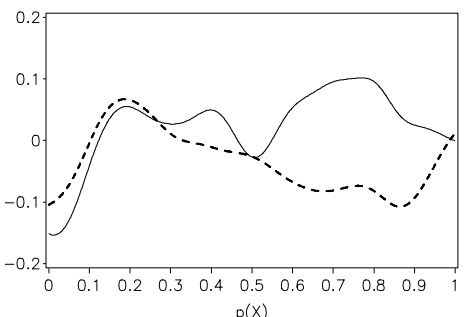

In ausserbetriebliche Arbeit

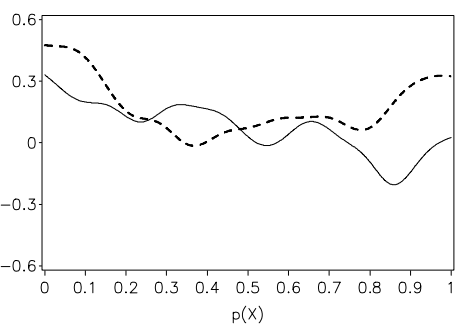

In RGV/HFF

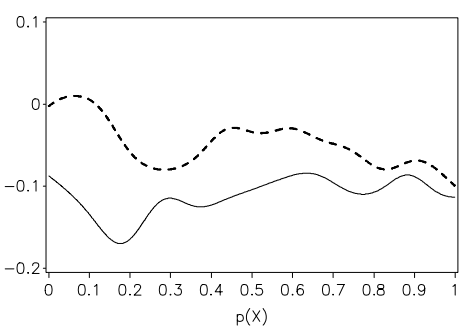

In Umsatz

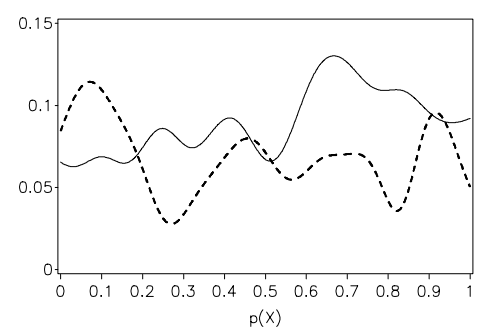

1. Nadaraya-Watson Schätzer mit Gauß'scher Kernfunktion und optimaler Bandbreite.

2. In den Plots ist die Ergebnisänderung $\left(\Delta y_{i}^{1}, \Delta y_{j}^{0}\right)$ von Teilnehmern (durchgezogene Linie) und Kontrollbetrieben (gestrichelte Linie) in Abhängigkeit von der Wahrscheinlichkeit der AUM-Teilnahme $(p(X))$ dargestellt. Der Effekt der Programmteilnahme auf die Teilnehmer (ATT) entspricht der Fläche zwischen beiden Linien.

Quelle: eigene Berechnungen, Datenquellen vgl. Tabelle A2 und A3. 
Abbildung A4: Mittelwerte der beobachteten (logarithmierten) $L F$ und der $L F$ Wachstumsrate (in \%) für alle in der Panelanalyse berücksichtigten Betriebe $(n=22.551)$
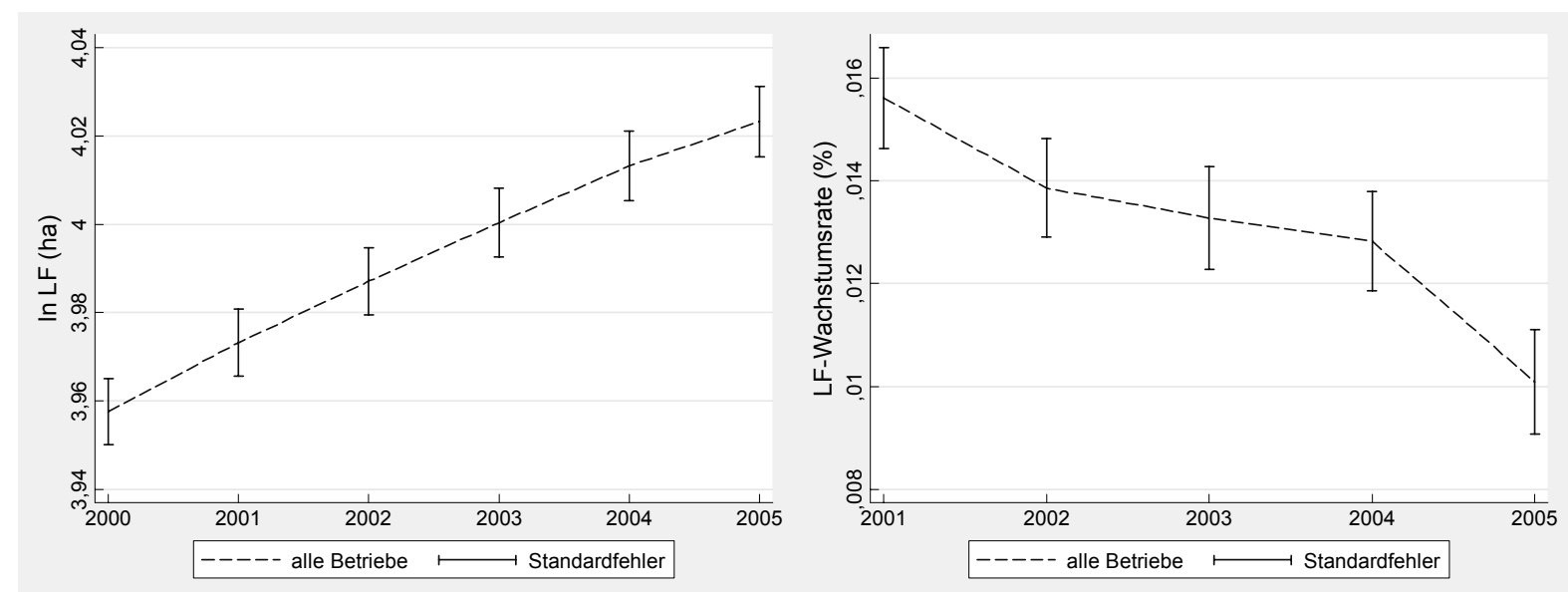

Quelle: eigene Berechnungen basierend auf LAND-DATA (WJ 1999/2000 bis WJ 2004/05).

Abbildung A5: Mittelwerte der beobachteten (logarithmierten) $L F$ und der $L F$ Wachstumsrate (in \%) für alle in der Panelanalyse berücksichtigten Betriebe $(n=22.551)$, differenziert nach AUM-Teilnehmern und AUMNichtteilnehmern
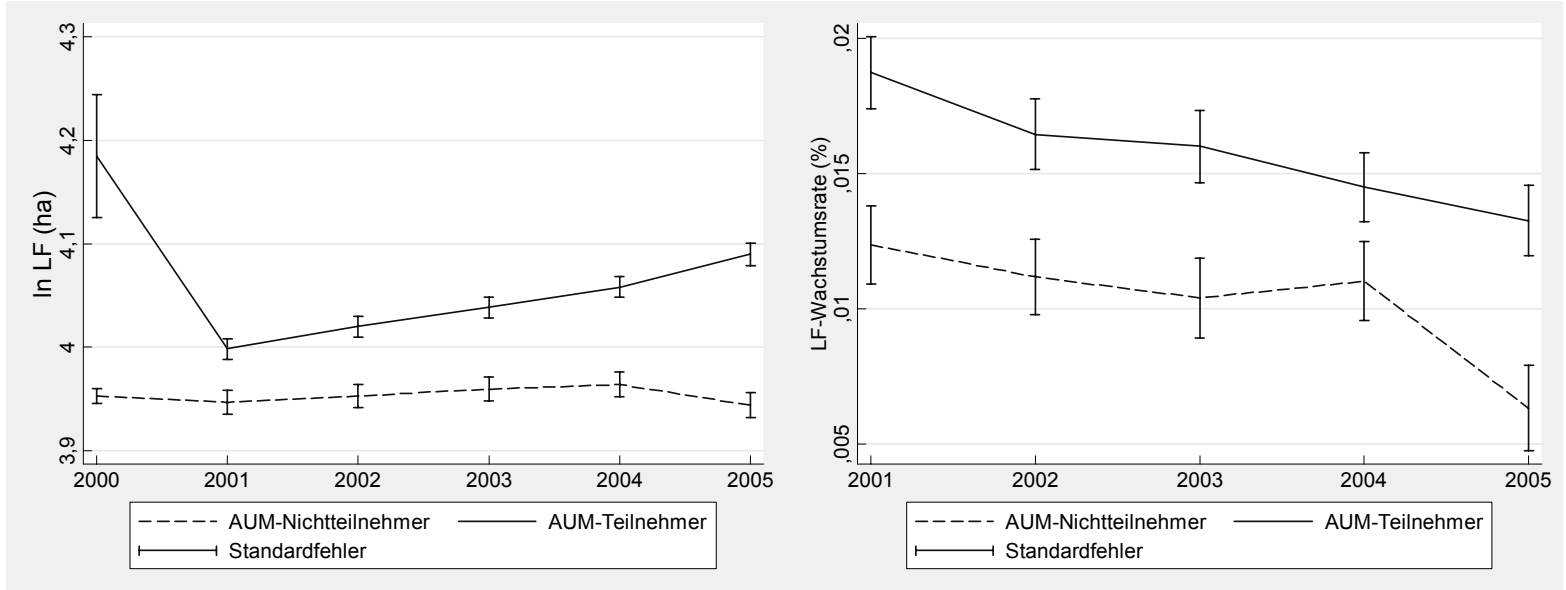

Quelle: eigene Berechnungen basierend auf LAND-DATA (WJ 1999/2000 bis WJ 2004/05). 
Abbildung A6: Mittelwerte der beobachteten (logarithmierten) $L F$ und der $L F$ Wachstumsrate (in \%) für alle in der Panelanalyse berücksichtigten Betriebe $(n=22.551)$, differenziert nach AZ-Teilnehmern und AZNichtteilnehmern
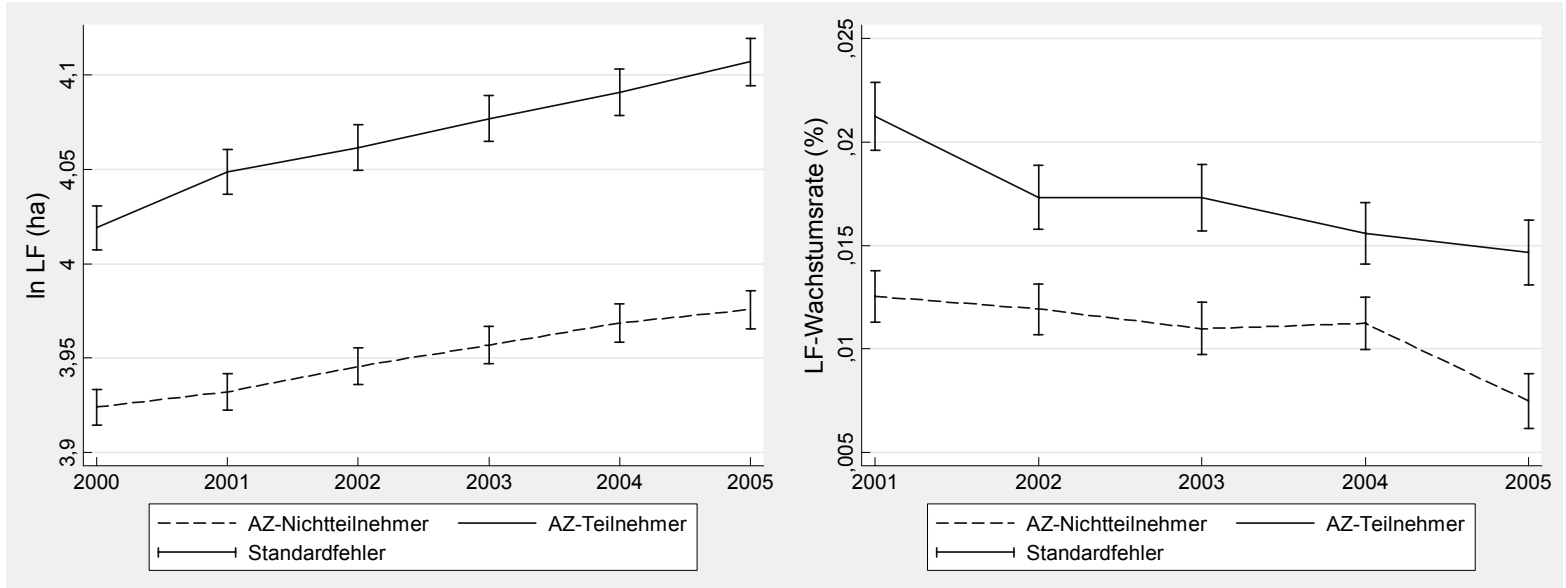

Quelle: eigene Berechnungen basierend auf LAND-DATA (WJ 1999/2000 bis WJ 2004/05).

Abbildung A7: $\quad$ Verteilung der AUM- und AZ-Teilnehmer nach der bedingten Wahrscheinlichkeit der Programmteilnahme
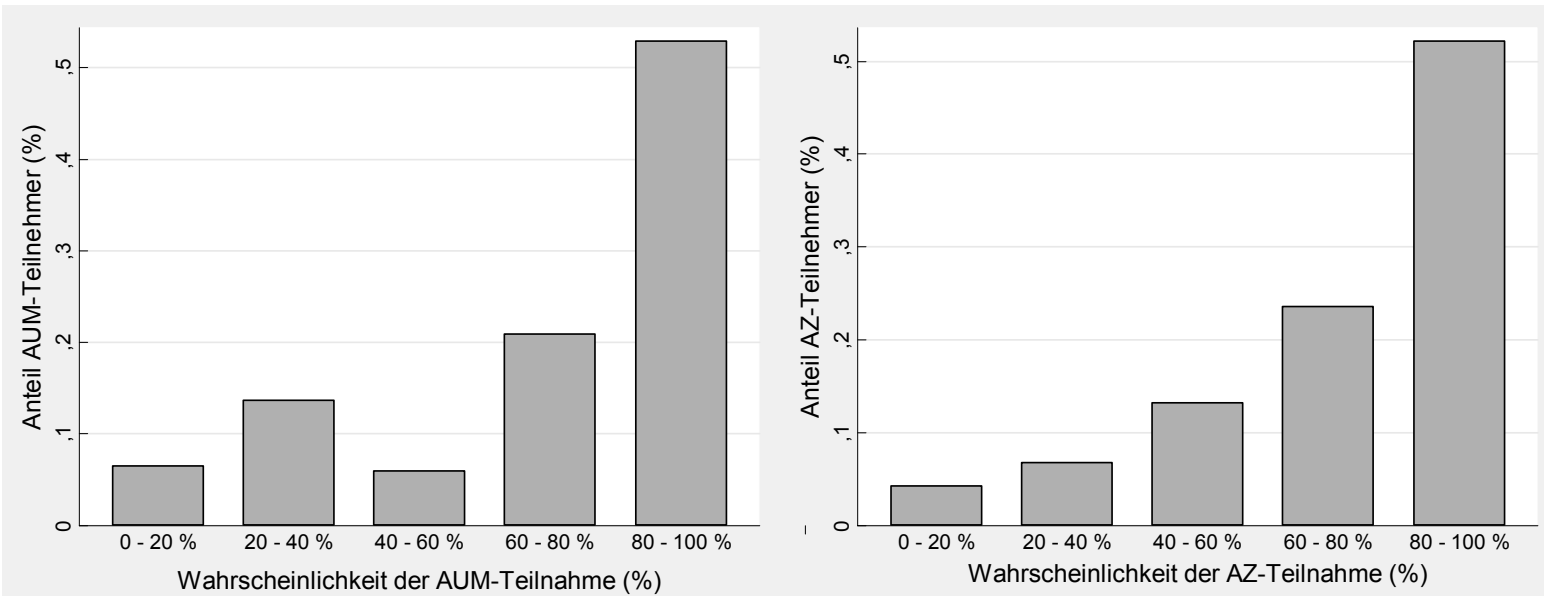

Die Wahrscheinlichkeit der AUM- und AZ-Teilnahme wurde für die Stichprobe der Panelanalyse 22.551 auf Basis von Probit-Schätzungen ermittelt. Die geschätzten Koeffizienten der Probit-Modelle sind in den Tabellen A8 (AUM) und A9 (AZ) dargestellt.

Quelle: eigene Berechnungen, Datenquellen vgl. Tabelle A2 und A3. 
Anhang

Karten 
Karte A1: $\quad$ Benachteiligte Gebiete in der Bundesrepublik Deutschland (75/268/EWG)

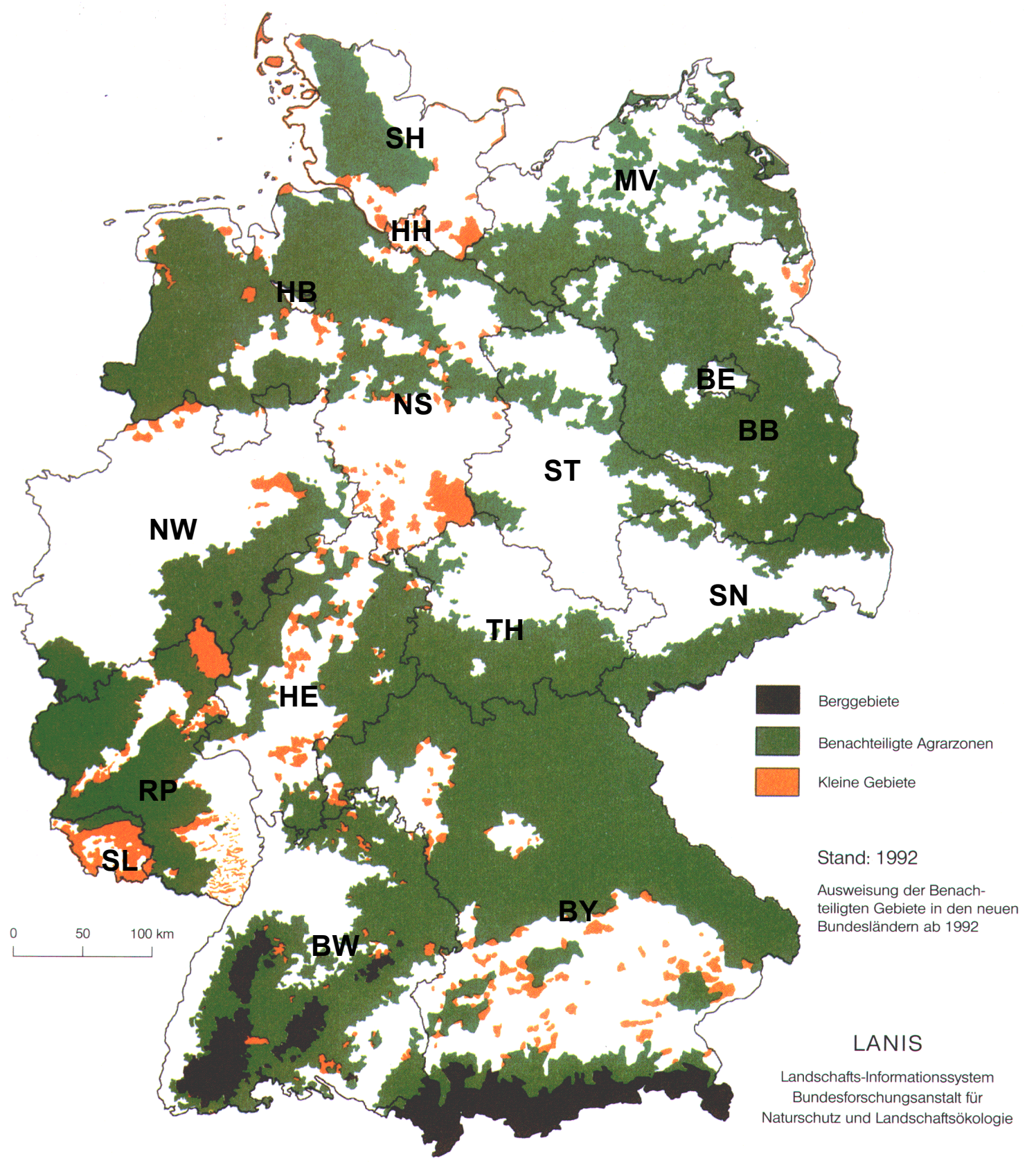

Quelle: BFNL (1993) 
